\title{
The structural acoustic properties of stiffened shells
}

\section{Luan, Yu}

\section{Published in:}

Acoustical Society of America. Journal

Link to article, DOI:

$10.1121 / 1.2932806$

Publication date:

2008

Document Version

Publisher's PDF, also known as Version of record

Link back to DTU Orbit

Citation (APA):

Luan, Y. (2008). The structural acoustic properties of stiffened shells. Acoustical Society of America. Journal, 123(5), 3063-3063. https://doi.org/10.1121/1.2932806

\section{General rights}

Copyright and moral rights for the publications made accessible in the public portal are retained by the authors and/or other copyright owners and it is a condition of accessing publications that users recognise and abide by the legal requirements associated with these rights.

- Users may download and print one copy of any publication from the public portal for the purpose of private study or research.

- You may not further distribute the material or use it for any profit-making activity or commercial gain

- You may freely distribute the URL identifying the publication in the public portal

If you believe that this document breaches copyright please contact us providing details, and we will remove access to the work immediately and investigate your claim 


\section{Session 1aID}

The Opening Ceremony will include a special welcome from the Vice-President of Ile de France Regional Council, addresses by National sponsors, and addresses by the Presidents of the Acoustical Society of America, the European Acoustics Association, and the French Acoustical Society. Musical interludes will be given and two plenary lectures will be presented.

\section{Plenary Lectures}

Introduced by George Frisk, Florida Atlantic University, USA

10:30

1aID1. How sound from human activities affects marine mammals. Peter Tyack (Woods Hole Oceanographic Institution, Applied Ocean Physics \& Engineering Dept., Woods Hole, MA 02543, USA, ptyack@whoi.edu)

Effects of sound on marine mammals have traditionally been defined either as injury or disruption of behavior. The earliest concern about behavioral disruption was that elevated noise could reduce the range of communication by masking faint signals. Few studies have documented this effect, but recent work emphasizes mechanisms animals use to compensate for elevated noise. Many studies have documented changes in behavior as a function of exposure, but it has proven difficult to relate these to effects on the welfare of individual animals or on the status of populations. Promising approaches to interpreting effects include avoidance of habitat, energetics of foraging, and applying models of anti-predator behavior to human disturbance. During the 1990s acoustic criteria for injury were designated based upon temporary hearing loss. Accumulating evidence of atypical mass strandings of beaked whales coincident with naval sonar exercises suggest that injury or death may result from behavioral responses of some species at lower exposure levels in some settings. A tagged beaked whale showed similar but weaker responses to experimental playback of a mid-frequency sonar sound compared to calls of killer whales, suggesting that anti-predator response may harm animals after exposure to levels of sound very unlikely to cause injury directly.

\section{Introduced by Luigi Maffei, Second University of Naples, Italy}

\section{1:10}

1aID2. New Trends in Aeroacoustics: From acoustic analogies to direct numerical simulations. Daniel Juvé (Ecole centrale de Lyon, 36 avenue Guy de Collongue, 69134 Ecully cedex, France, daniel.juve@ec-lyon.fr)

Modern aeroacoustics started in the early 1950's when Lighthill developed his famous acoustic analogy in an attempt to understand, and reduce, the terrifying noise generated by jet aircrafts. For nearly 50 years the subject of aerodynamic sound was dominated by approaches based upon this analogy or variants of it. Recently, the availability of powerful computing facilities combined with the development of numerical algorithms specially designed to simulate sound propagation over large distances has paved the way for "a second golden age of aeroacoustics" (to quote Lighthill himself). In this talk we will first give an overview of this evolution from acoustic analogies to computational aeroacoustics (CAA). Typical illustrations of the CAA approach will then be presented and applications for transportation systems will be discussed. 


\title{
Session 1pAAa
}

\section{Architectural Acoustics and Noise: Acoustics of Open-Plan Spaces I}

\author{
Kenneth P. Roy, Cochair \\ Armstrong Building Products, 2500 Columbia Ave, Lancaster, PA 17603, USA \\ Valtteri O. Hongisto, Cochair \\ Finnish Institute of Occupational Health, Lemminkäisenkatu 14-18 B, Turku, 20520, Finland \\ Stephen Sinclair, Cochair \\ Centre for Interdisciplinary Research in Music Media \& Technology, Schulich School of Music, McGill Univ., \\ Montreal H3A IE3, QC, Canada
}

\section{Invited Papers}

1:00

1pAAa1. Spatial decay, behaviour and space planning models European and International standards on open office acoustics. Pierre Chigot (Saint Gobain Ecophon SA, box 30030, 60291 Rantigny, France, pierre.chigot@saint-gobain.com)

Recent standardisation work on open plan offices acoustics is characterized by a shift in the way room acoustics is handled. Spatial decay (expressed through rate of spatial decay per doubling of distance, DL2) is preferred to temporal decay (expressed through reverberation time, RT). The complexity of open plan office acoustic design results from the fundamental contradiction of communication and concentration. DL2 reflects better the challenges of sound control in such rooms, which is basically sound propagation control. Beyond, this contradiction, specific space analysis models and tools can help to visualize the acoustic interactions between workstations, teams and departments accommodated in the same room. These models are now ready to be included in standardization work. International technical standard for field measurements of DL2 integrates new descriptive models for open plan office acoustics, taking into account geometric proportions, presence of screens and furniture as well as group behaviour and speech characteristics, such as increased energy contents at low frequencies, voice levels, raised hearing sensitivity at high frequencies. Also, normative guidelines from Netherlands and France integrating this approach will be presented.

$1: 20$

1pAAa2. Acoustics and sustainable design in exposed structures. Kenneth P. Roy (Armstrong Building Products, 2500 Columbia Ave, Lancaster, PA 17603, USA, kproy@armstrong.com), Anita L. Snader (Armstrong World Industries, 2500 Columbia Ave, R\&D Bldg. 5, Lancaster, PA 17604, USA, alsnader@armstrong.com)

Two very important emerging architectural trends in the USA are to 1) 'open up' the space design as exposed structures, and 2) incorporate sustainable design into building interiors. We as acoustic designers would like to know the consequences of both on acoustics performance and occupant satisfaction and work performance. The first concern is addressed in a study by CISCA evaluating the effects of ceiling plenum vs. exposed structures in both office and retail spaces. And the second issue of compatibility of sustainable design objectives with acoustic performance is addressed by a pertinent field survey by the Center for the Built Environment (CBE at Univ. of CA, Berkeley) on occupant satisfaction and performance. The issue of the availability and use of 'green' acoustic building materials that not only meet sustainability and energy goals, but also serve to ensure that our buildings actually work for their intended purposes will be addressed. Finally, the increased awareness of the acoustic impact of 'green' design on occupant satisfaction is being seen in the evolution of LEED and other 'green' rating systems.

\section{$1: 40$}

1pAAa3. Differences in perception of noise and privacy in different office types. Christina Bodin Danielsson (The Royal Institute of Technology, KTH, Roslagsgatan 37, 11354 Stockholm, Sweden, c.danielsson@comhem.se)

Differences between office types with regard to their architectural and functional features may have an impact on the employees' disturbance by noise and perception of privacy. These aspects may in its turn have an impact on employees' satisfaction and psychological responses to the office environment. In a study 469 employees in 26 different companies have rated their satisfaction with the office environment in seven different office-types: cell-office, shared room office, small open plan office, medium open plan office, large open plan office, flex-office and combi-office. This paper discusses the results concerning the employees' perception of noise and privacy and put the results in relation to the two different aspects of privacy, visual and acoustic privacy, and the role of personal control. In the statistical analysis adjustments for potential confounders as age, gender, job rank and line of business were done, which are known to have an influence on perception and satisfaction. Substantial differences between employees in different office-types were found. The fact that there were such differences between different types of offices that mean sharing of workspace and work facilities was a surprise. Architectural and functional features of the offices are discussed as the main exploratory factors for these results. 


\section{Contributed Paper}

\section{2:00}

1pAAa4. Effect of sound masking on workers in an open office. Annu Haapakangas (Finnish Institute of Occupational Health, Lemminkäisenkatu 14-18 B, 20520 Turku, Finland, annu.haapakangas@ttl.fi), Valtteri O. Hongisto (Finnish Institute of Occupational Health, Lemminkäisenkatu 14-18 B, 20520 Turku, Finland, valtteri.hongisto@ttl.fi)

Appropriate masking sound is necessary for reaching acceptable speech privacy in open offices. However, the electronic masking systems have not become very popular although the importance of masking is emphasized in design guidelines worldwide. One reason may be that very few scientific field experiments have been published in this area. The aim of this pilot study was to investigate the effects of artificial masking sound on workers in a small department of 15 workers. Measurements and questionnaires were conducted before and after launching the system. Masking sound $44 \mathrm{dBA}$ was produced using centralized pink noise generator and ceiling loudspeakers. The spectrum reminded ventilation noise. Initial background noise level was $36 \mathrm{dBA}$. Acoustic measurements showed that masking sound reduced the radius of distraction $\mathrm{rD}$ from $15 \mathrm{~m}$ to $7 \mathrm{~m}$, i.e. acoustic privacy improved significantly. The questionnaire revealed several positive changes attributable to masking. Distraction caused by speech and other varying office sounds was reduced. Noise-related stress was reduced. Evaluations of acoustic environment, speech privacy and self-rated work efficiency were improved. The results showed no adverse effects of masking on workers.

\section{Invited Papers}

\section{$2: 20$}

1pAAa5. Speech Privacy Options for the Open Ceiling Office Environment. Dana S. Hougland (Shen Milsom Wilke, Inc., 1822 Blake Street, Suite 2A, Denver, CO 80202, USA, dhougland@smwinc.com)

The popularity of the open ceiling and loft office environment continues to grow. The design and effectiveness of sound masking systems in an open office environment are well established for traditional office spaces with acoustically absorptive ceilings systems. Aesthetic preferences for open ceilings with minimal ceiling treatment popular in the creative reuse of older buildings and more minimalist industrial spaces limit the sound masking options available to achieve an acceptable level of speech privacy in an open office environment. Traditional sound masking systems are often subjectively viewed as harsh, unpleasant or visually inappropriate. Several options for alternative designs are evaluated as to the evenness of coverage, effectiveness in achieving an appropriate level of speech privacy, aesthetic integration into the environment and subjective impression of the occupants.

$$
\text { 2:40 }
$$

1pAAa6. Effective sound masking for speech privacy in open plan offices. Joel Lewitz (Rosen Goldberg Der \& Lewitz, Inc., 1100 Larkspur Landing Circle, Suite 354, Larkspur, CA 94939, USA, jlewitz@RGDLacoustics.com)

For over 30 years acousticians have studied, measured and evaluated open plan offices to improve speech privacy and freedom from distraction. This paper addresses the relative importance of the many factors affecting speech privacy in open plan offices. Adjusting the background noise level at the receiver has proven to be a very powerful and relatively inexpensive method to optimize the acoustical environment in open plan offices. However, sound masking systems are not always properly tuned to achieve the maximum benefit. In this paper, the importance of the shape, smoothness and level of the masking sound spectrum are discussed and demonstrated with case studies. Recent legislation in the U.S., such as the Healthcare Insurance Portability and Accountability Act of 1996, also known as HIPAA, has broadened speech privacy concerns from open plan to closed plan. Sound masking is an attractive option for both configurations. Differences in sound masking design and tuning between open plan and closed plan are explored in this paper.

\section{3:00}

1pAAa7. Room acoustical measures for open plan spaces. Erling Nilsson (Saint-Gobain Ecophon AB, Box 500, SE-260 61 Hyllinge, Sweden, erling.nilsson@ecophon.se), Björn Hellström (ÅF-Ingemansson AB, Box 47321, SE-100 74 Stockholm, Sweden, Bjorn.Hellstrom@afconsult.com), Björn Berthelsen (Saint-Gobain Ecophon AB, Box 500, SE-260 61 Hyllinge, Sweden, bjorn .berthelsen@ecophon.se)

In an ongoing Nordic cooperation project the acoustical conditions in open-plan spaces are investigated. Of special interest is to find suitable acoustical parameters that reveal the acoustical conditions in these spaces and also are of importance for the subjective perceived acoustics. Measurements have so far been carried out in five open plan offices accompanied with an inquiry gathering the subjective judgments by the staff. A program for the acoustical measurements was designed specifying how to perform the measurements, which type of parameters to measure and how to report the results. The acoustical parameters included in the measurements are Reverberation time T20, Early Decay Time (EDT), Deutlichkeit (D50), Speech transmission index (STI), Speech intelligibility index (SII), Privacy Index (PI), Rate of spatial decay of sound pressure levels per distance doubling (DL2), Excess of sound pressure level with respect to a reference curve (DLf), background noise levels in occupied and unoccupied offices. The behavior of these parameters will be discussed and suitable parameters for evaluation of open plan spaces will be suggested.

\section{3:20-3:40 Break}

\section{3:40}

1pAAa8. Characterization of acoustics in open offices - four case studies. Jukka S. Keränen (Finnish Institute of Occupational Health, Lemminkäisenkatu 14-18 B, 20520 Turku, Finland, jukka.keranen@ttl.fi), Petra Virjonen (Finnish Institute of Occupational Health, Lemminkäisenkatu 14-18 B, 20520 Turku, Finland, petra.virjonen@ gmail.com), Valtteri O. Hongisto (Finnish Institute of Occupational Health, Lemminkäisenkatu 14-18 B, 20520 Turku, Finland, valtteri.hongisto@ttl.fi)

Acoustic design in open offices aims to reduction of distractions and improvement of speech privacy. Room acoustical design includes high room absorption, high and absorptive screens and appropriate masking sound level. The aim of this study is to show, how these individual design components can affect on room acoustics, using modern room acoustical descriptors. Individual acoustical remedies were studied in four case studies independently. The implemented room acoustical changes were increased ceiling absorption, 
sound-absorptive screens, curtains between workers and increased masking sound level. Radius of distraction, $\mathrm{r}_{\mathrm{D}}$, spatial decay rate of speech, $\mathrm{DL}_{2}$, and speech level at $4 \mathrm{~m}$ from speaker, $\mathrm{L}_{\mathrm{p}, \mathrm{S}, 4 \mathrm{~m}}$, were determined before and after the room acoustical change. These three simple and robust single-number parameters are recommended to characterize the acoustic conditions of open offices because they react logically to room acoustical changes.

\title{
Contributed Papers
}

4:00

1pAAa9. Possibilities for acoustic improvement of the far field in open-plan offices. Catrin Humer (TGM Federal Institute of Technology, Wexstrasse 19-23, 1200 Vienna, Austria, catrin.humer@tgm.ac.at), Herbert Muellner (TGM Federal Institute of Technology, Wexstrasse 19-23, 1200 Vienna, Austria, herbert.muellner@tgm.ac.at)

An increase in open-plan offices has occurred recently. This makes it necessary to examine and, if necessary, to improve the acoustic conditions in the planning phase. With acoustic simulations it is possible to verify the acoustic quality of offices without much increase in money and time. In order to get the needed absorption area of the room to reduce the reverberation time and the sound level, open-plan offices are usually partitioned with screens. To improve the acoustics in the close range, special conditions, which mainly concern the height, have to be met. Also the absorption and the height of the ceiling have a strong influence on the acoustic quality of the neighbouring workstations. In the far field additional planning bases apply - particularly to raise the horizontal absorption and to get the optimal screening effect. This report highlights the possibilities of the acoustic improvements in the far field in open-plan offices and shows examples of simulated and improved options.
$4: 20$

1pAAa10. Modeling the sound field of open spaces using a diffusion equation model. Yun Jing (Rensselaer Polytechnic Institute, Greene Building, School of Architecture, 110 8th Street, Troy, NY 12180, USA, jingy@rpi.edu), Ning Xiang (Rensselaer Polytechnic Institute, Greene Building, School of Architecture, 110 8th Street, Troy, NY 12180, USA, xiangn@rpi.edu)

Steady-state and transient sound field modeling of open spaces, such as urban squares, courtyard-type traditional Chinese theaters, is discussed in this paper. It is well known that the application of the statistical roomacoustic theory is strongly limited in this case, because of the high nonuniformity of the absorption coefficient. In this study, a diffusion equation along with a recently proposed modified boundary condition [Y. Jing and N. Xiang, J. Acoust. Soc. Am., 123, 2008, 145-153] is used to predict the acoustic characteristics of open spaces, in terms of both sound pressure level distributions and reverberation times. Three different types of open spaces are presented, including urban squares, quasi-cubic open rooms, and urban streets. This paper will compare the simulation results with the experimental results conducted in all these three cases (scale model), to verify the validity and efficiency of the diffusion equation model.

MONDAY AFTERNOON, 30 JUNE 2008

ROOM 252B, 1:00 TO 7:20 P.M.

\section{Session 1pAAb}

\section{Architectural Acoustics: Acoustics of Concert Halls I}

\author{
Takayuki Hidaka, Cochair \\ Takenaka R\&D Institute, 1-5-1, Otsuka, Inzai, Chiba, 270-1395, Japan \\ Daniel E. Commins, Cochair \\ Commins Acoustics Workshop, 15 rue Laurence Savart, Paris, 75020, France
}

Invited Papers

1:00

1pAAb1. From backward integration to number-theoretic diffusors. Manfred Schroeder (University of Goettingen, Rieswartenweg 8, 37077 Goettingen, Germany, mrs17@aol.com)

As a student of physics at Göttingen I was interested in the statistics of electromagnetic resonances in metallic cavities when Erwin Meyer offered me a scholarship in concert-hall acoustics. So we compromised and my thesis eventually appeared under the double heading "The statistical parameters of the frequency responses of large enclosures. Experiments with electromagnetic waves".-After moving to Bell Laboratories in New Jersey, I continued to explore, with Heiner Kuttruft, frequency responses in concert halls by digital simulation, confirming my earlier theory that, above a critical frequency, these responses are just "noise" in the frequency domain. Later I dabbled in anti-feedback circuits and explored, with Ben Logan, artificial reverberation that spawned a minor industry-now dubbed sound scapes (first realized by John Chowning) and virtual reality. Then came the work (with Gerhard Sessler, Jim West, Bishnu Atal and Carol Bird) on Philharmonic Hall in New York, which lead to a new method of measuring reverberation time ("backward" integration) and new surface structures, based on number theory, for better sound diffusion. (The idea came to me during a talk by André Weil on "Gauss Sums" during the celebration of the 200th anniversary of Gauss' birth.) 
1pAAb2. Echo suppression effect and coloration of periodic-type diffusers. Masahiro Toyoda (Pioneering Research Unit, B104, Kyoto University Katsura, Nishikyo-ku, 615-8530 Kyoto, Japan, masahiro.toyoda@kupru.iae.kyoto-u.ac.jp), Tomohiro Furukawa (Kajima Corporation, Akasaka 6-5-30, Minato-ku, 107-8502 Tokyo, Japan, furuktom@kajima.com), Daiji Takahashi (Pioneering Research Unit, B104, Kyoto University Katsura, Nishikyo-ku, 615-8530 Kyoto, Japan, tkhs@ archi.kyoto-u.ac.jp)

Walls and ceilings are often designed to suppress undesirable echoes. For that purpose, walls and ceilings are given absorptive surfaces which absorb sound energy or uneven surfaces which provide wave diffusion. While it is not difficult to predict the degree of echo suppression effects in the case of absorptive surfaces, it is generally difficult to do that in the case of uneven surfaces. In addition, if the surfaces have periodicity, unintended effects called coloration can distort tonal characteristics of the sound field. Thus, many acoustic designers would hesitate to employ this kind of periodic-type diffusers. However, there are some cases where it is necessary to suppress echoes without any energy loss. Periodic-type diffusers have the advantages of easy estimation of diffusion properties, simple method of design, and ready availability. In this study, a subjective experiment with simulated stimuli was carried out to clarify the echo suppression effect of the periodic-type diffusers from the viewpoint of reflected energy level. Although coloration interfered in subjective judgments in some cases of the experiment, it is shown that the periodic-type diffusers have echo suppression effect if coloration does not occur. This study is also devoted to discussion about the cause of the coloration.

\section{$1: 40$}

1pAAb3. Experimental analysis of the acoustical behaviour of Musikverein in concert and ballet configurations. Angelo Farina (University of Parma, Via delle Scienze 181/A, Industrial Engineering Dept., 43100 Parma, Italy, farina@unipr.it), Daniel E. Commins (Commins Acoustics Workshop, 15 rue Laurence Savart, 75020 Paris, France, d.commins@comminsacoustics.com), Nicola Prodi (Engineering Dept. - Univ. of Ferrara, Via Saragat, 1, 44100 Ferrara, Italy, nicola.prodi@ unife.it)

Musikverein is among the best concert halls in the world. But the hall is also employed for open-to-the-public ballets. All seats in main stalls are removed, and packed under the wooden floor thanks to a large opening. Detailed acoustical measurements have been performed, with the aim of evaluating the different acoustical behavior with and without the seats. Furthermore, it was attempted to assess the contribution of the air volume under the floor, as a possible "resonant chamber", with volume and absorption significantly different when it is filled with packed seats. The results did show that the main acoustical parameters vary significantly between the "concert" setup and the "public ballet" setup, which is more reverberant, with lesser clarity and more envelopment. Regarding the hypothesis that the volume under the floor has some effect on the acoustical behaviour of the room, the experimental results excluded that this is possible. Furthermore, measurements with accelerometers and pressure/velocity probes did not reveal any difference between the behaviour of the "openable" part of the floor and the surrounding "fixed" floor. So it was concluded that the large difference in acoustical behaviour is simply caused by the removal of the seats, and by changes in fittings of the stage.

2:00

1pAAb4. Acoustical considerations in the design for 'La Philharmonie de Paris'. Harold Marshall (Marshall Day Acoustics LTD, P O Box 5811, Wellesley St., 1000 Auckland, New Zealand, harold.marshall@marshallday.co.nz), Joanne O. Valentine (Marshall Day Acoustics LTD, P O Box 5811, Wellesley St., 1000 Auckland, New Zealand, joanne.valentine@marshallday.co.nz), Thomas Scelo (Marshall Day Acoustics LTD, P O Box 5811, Wellesley St., 1000 Auckland, New Zealand, thomas.scelo@marshallday.co.nz)

The 2006 competition instructions for "La Philharmonie de Paris" included a detailed acoustical specification and the general requirement that the design should be a "new typology". The early and late sound fields respectively should be controlled independently. This paper gives an account of the acoustical aspect of the winning design, its relationship to the architecture at the competition phase, and the methodology for developing the design in subsequent phases. The new typology developed here is called the "bicameral adaptable concert hall". There are partial precedents for the concepts advanced and these will be identified. Reference is also made to a related paper (at this conference) which discusses the issue of coupling between the acoustical spaces.

$$
\text { 2:20 }
$$

1pAAb5. Acoustical design of Shenzhen Concert Hall, Shenzhen China. Keiji Oguchi (Nagata Acoustics Inc., 2130 Sawtelle Blvd., Suite 307A, Los Angeles, CA 90025, USA, oguchi@nagata.co.jp), Yasuhisa Toyota (Nagata Acoustics Inc., 2130 Sawtelle Blvd., Suite 307A, Los Angeles, CA 90025, USA, toyota@nagata.co.jp)

The Shenzhen Concert Hall complex opened in Shenzhen, China on October 12, 2007. The complex contains the Symphony Hall with 1,800 audience seats, the Theater Studio with 400 - 580 seats and some rooms for rehearsing. The Symphony Hall has a vineyard configuration with a steep audience block arrangement, called "canyon terrace". At the widest point of the audience seating, the room spans $45 \mathrm{~m}$, is $60 \mathrm{~m}$ long and has a ceiling height of $25 \mathrm{~m}$. The polycarbonate ensemble reflector panels are suspended at the height of approx. $15 \mathrm{~m}$ above the stage. The acoustical design and characteristics of the Concert Hall are reported.

\section{2:40}

1pAAb6. A comparison between shoebox and non-shoebox halls based on objective measurements in actual halls. Takayuki Hidaka (Takenaka R\&D Institute, 1-5-1, Otsuka, 270-1395 Inzai, Chiba, Japan, hidaka.takayuki@takenaka.co.jp), Leo L. Beranek (975 Memorial Drive, Suite 804, Cambridge, MA 02138, USA, beranekleo@ieee.org), Noriko Nishihara (Takenaka R\&D Institute, 1-5-1, Otsuka, 270-1395 Inzai, Chiba, Japan, nishihara.noriko@takenaka.co.jp)

In recent papers, the sound absorption by chairs and audiences were determined for shoebox (SB) and non-shoebox (NSB) types of concert halls (Beranek, JASA 120, 1399 (2006); Hidaka, Proc. ICA2007). The purpose of this paper is to examine objective differences in the sound fields in these two hall types. This investigation follows reports of music goers that the average subjective sound quality 
in NSB and SB halls differs appreciably and that there are greater subjective differences in sound quality from one seating location to another in NSB halls. Special attention is given to the temporal and amplitude structure in the early part (before $80 \mathrm{msec}$ ) of the impulse responses measured at various seat positions in six well-known halls (three SB and three NSB). To the extent the data permit, the usual objective parameters are also analyzed, both averaged and at various seat locations, in a total of 12 halls: reverberation time, early decay time, C80 (3-band), interaural cross-correlation coefficient (3-band middle frequency early before $80 \mathrm{~ms}$ ), initial-time-delay-gap, bass ratio, and strength factors at low- and mid-frequencies. The differences found are discussed with reference to the subjective reports.

\section{3:00}

1pAAb7. Changing seating arrangements of the orchestras - a challenge for the concert hall design. Jürgen Meyer (Bergiusstr. 2a, D-38116 Braunschweig, Germany, juergen.meyer-bs@t-online.de)

For the future of concert hall design, two aspects will play an increasing role: the search for new shapes created by the architects and changing seating arrangements preferred by the conductors. Whereas in the last decades, acoustic consultants could assume that e.g. celli and double basses were positioned on the right side of the stage, they will to have consider now that more and more conductors - particularly those of high international reputation - go back to the classic seating arrangement (celli in the center, 2nd violins on the right side. Among others, this distinct tendency leads to the following questions: Which are the tonal (acoustic) advantages of the German seating arrangement and by which roomacoustical means can they been supported? In which directions - seen from the player - exist important reflection areas for timbre, clarity and spaciousness? How should risers on the stage be designed for improving the listeners' sound impression and supporting the musicians' mutual hearing? Do celli and basses need podium areas capable of vibrating? Furthermore, where are critical areas and delay times for generating echos or erroneous localization of French horns or other winds? These questions shall be discussed from physical and psychoacoustic aspects.

1pAAb8. Acoustical analysis of a variable roof configuration concert hall: The São Paulo Hall. Roberto A. Tenenbaum (Instituto Politecnico, Universidade do Estado do Rio de Janeiro, Rua Alberto Rangel, S/N, 28630-050 Nova Friburgo, Brazil, tenenbaum@iprj.uerj.br), Lenine V. Oliveira (Universidade Federal do Rio de Janeiro, Av. Marechal Trompowsky S/N, 21970-000 Rio de Janeiro, Brazil, leninevas@ig.com.br), Swen Müller (Instituto Nacional de Metrologia e Controle da Qualidade, Av. N.S. das Graças, S/N, 21000-000 Xerem, Brazil, smuller@inmetro.gov.br)

The São Paulo Hall (SPH) is recognized by musicians and musical critics in general by its good acoustical quality. It has, as an important feature, variable acoustics due to its movable roof, which is employed by musicians to tune the room according to the musical style. This work addresses the acoustical quality of SPH and its dependence on the roof configuration. Impulse responses for one source, eight microphone and two dummy head positions, using the sweep-sine technique with pre-emphasis, were obtained for seven roof configurations. Some details of the measurement procedure to ensure good accuracy are discussed. The measurement results were then used to compute the main room acoustical quality parameters, for each octave band between 63 and $8000 \mathrm{~Hz}$. The spatial average and deviation of these data are presented as a function of the frequency band. The influence of the roof configurations on the obtained acoustical parameters is also discussed. Furthermore, comparative plots among SSP at the chosen roof configurations and two famous and representative halls from the classical and romantic periods are reported and discussed. It is concluded that the movable roof is very effective in modifying the room acoustics and that the musician's choices are quite good.

\section{3:40}

1pAAb9. The acoustic design of the Elisabeth Murdoch Hall, Melbourne, Australia. Raf Orlowski (Arup Acoustics, St Giles Hall Pound Hill, CB3 0AE Cambridge, UK, Raf.Orlowski@arup.com)

The Elisabeth Murdoch Hall is a 1000 seat recital space which will be completed in the latter half of 2008. The overall geometry is based on a classical shoebox shape to provide acoustic excellence although the internal appearance is wholly contemporary. The walls and ceiling of the hall are clad in timber panelling which is stepped in and out and also modulated to provide diffusion at mid and low frequencies. In addition, the surface layer of the panels is grooved or cut away to form an abstract pattern which is both decorative and diffusing at high frequencies. A notable aspect of the hall is that it has no orchestral reflector. The important early reflections for good orchestral ensemble are provided by articulating and shaping the surfaces around the stage. Although the hall is primarily for recitals of classical music, provision is made for amplified music by introducing acoustic drapes.

\section{4:00-4:20 Break}

1pAAb10. Trends in preference, programming and design of concert halls for symphonic music. Anders C. Gade (Dept. of Acoustic Technology, Technical University of Denmark, Building 352, DK 2800 Lyngby, Denmark, acg@ oersted.dtu.dk)

This paper discusses the evolution in taste regarding concert hall acoustics and how this can be reflected in the new halls being built today. The clients' and listener's preferences are not only based on listening in existing halls; but also on listening to reproduced music recorded with microphones close to the orchestra and with artificial reverberation added. The result may be a desire for higher clarity as well as a more full reverberation than what is found in most existing halls. Without being very specific in the brief regarding geometrical detailing - which is not desirable as it will limit architectural freedom and evolution - we can only specify a desire for acoustic conditions in this direction by setting targets for standardized objective room acoustic parameters. In this paper, measured ISO 3382 data are used to illustrate typical differences between "live" and recorded concert experiences, and it is seen how visionary hall designs over the last four decades tend to move the acoustics of halls in the same direction. Finally, it is suggested how target values for ISO 3382 parameters - in spite of their limitations - can be set up to drive the design in such a direction. 
1pAAb11. Describing measurement uncertainties in room acoustics with the concept of GUM. Ingo Witew (Institute of Technical Acoustics, Templergraben 55, 52056 Aachen, Germany, Ingo.Witew@akustik.rwth-aachen.de), Renzo Vitale (Institute of Technical Acoustics, Templergraben 55, 52056 Aachen, Germany, renzo.vitale@akustik.rwth-aachen.de)

Considering the uncertainties in room acoustical measurements is a crucial step towards quality evaluation, comparability between different measurements methods and instrumentation. In the preceding discussion it was shown that the concepts specified in the "Guide to the expression of Uncertainty in Measurements" (GUM) can well be applied for room acoustical measurement tasks. In this initiatory step a detailed analytical formulation of a model function was avoided for reasons of simplicity and practicability. The waiving of complex modeling, however, requires comprehensive series of measurements to assess the measurement uncertainties. In this paper it is discussed how modeling can reduce such requirements. A special focus is placed on the influence of the directional radiation properties of sound sources.

5:00

1pAAb12. Simplified Room Acoustic Measurements. Tor Halmrast (Statsbygg, Biskop Gunnerus gt 6, pb 8106, Dep, NO-0032 Oslo, Norway, th@statsbygg.no), Anders Buen (Brekke \& Strand akustikk as, Hovfaret 17, 0275 Oslo, Norway, Anders.Buen@bsakustikk.no)

Standardised room acoustic measurements are needed for concert halls etc., but they imply heavy equipment. Thus, many interesting rooms are not measured. Most musicians (and even some acousticians) find that clapping and shouting in a room/hall might give an overview of the room acoustics. In the literature we even find that acoustic parameters are derived from scratchy recordings of music. Modern handheld high quality recording technology and possibilities of analysing sound files gives that one should investigate simpler (non standardised) measurements for simpler rooms/halls. This paper gives analysis of Impulse-Responses recorded from balloons, paper bags, clapping, compared with measurements taken with more academic measurement equipment.

\section{Contributed Papers}

$5: 20$

1pAAb13. Acoustics of concert halls with organs in Russia: trends and problems. Pavel Kravchun (Moscow State University, Faculty of Physics, Department of Acoustics, Box 15, 125130 Moscow, Russian Federation, gedackt@mail.ru)

Over the past decades the organ music has become one of the most popular kinds of classical music in Russia. About 30 new organs were built in concert halls, and several old instruments were restored or rebuilt. Acoustical properties of 15 well-known Russian concert halls with organs (Moscow, St. Petersburg, Perm, Kazan, Astrakhan, etc.) are presented and considered in the paper. In many cases acoustics in the concert halls is not so good for the solo organ music. The reasons are discussed. Acoustics of the Russian concert halls with adjustable acoustics is considered (Moscow, Kazan). It was noted that the "optimum" reverberation time recommended by different acousticians for organ music had a tendency to be longer and longer in the 20th century. Values of the average sound absorption coefficient for organ front were estimated. Some problems in organ-building connected with the concert hall acoustics are discussed.

\section{$5: 40$}

1pAAb14. Dexterity and Spirit: The Peter Kiewit Concert Hall at the Holland Performing Arts Center. Dawn Schuette (Threshold Acoustics, 53 W. Jackson Blvd, Suite 1734, Chicago, IL 60604, USA, dschuette @ thresholdacoustics.com)

Bathed in natural light, the Peter Kiewit Concert Hall is the focal point of the architecturally innovative Holland Performing Arts Center. The space features cast plaster panels for acoustic diffusion, a tunable canopy over stage for acoustic support, and natural felt banners for reverberation and reflection control. The space transforms easily from an orchestral concert setting to an environment friendly to amplified sound, allowing orchestral music one night to seem as naturally suited to the room as amplified music the following evening. The world-class concert hall acoustics provided by this venue meld seamlessly with the amplified use as well resulting in a unique venue for the music community. This hall is an excellent starting point for a continued discussion about how to successfully incorporate variability in high quality acoustic venues. [Work completed while author was employed as a Principal Consultant at Kirkegaard Associates.]
6:00

1pAAb15. A new symphonic hall, Musikhuset Aarhus, Denmark. Carsten Lemvigh Fog (COWI A/S, Parallelvej 2, DK 2800 Kgs. Lyngby, Denmark, clf@cowi.dk), Richard M. Ballinger (COWI A/S, Odensevej 95, DK 5260 Odense S, Denmark, rmb@cowi.dk)

During the past 3 years Musikhuset Aarhus in Denmark has been enlarged by an area of approximately $17.000 \mathrm{~m}^{2}$ to a new total area of approximately $34.000 \mathrm{~m}^{2}$. The new part of the complex contains a symphonic concert hall for Aarhus Symphonic Orchestra with 1200 seats, a hall for rythmic contemporary music with 600 seats and a hall for classical music with 160 seats. The complex also houses the new premises for Aarhus Academy of Music. Important design considerations will be highlighted in the paper and the results of the preliminary and final computer modeling of room acoustics will be presented using examples of auralisation. In conclusion the room acoustic parameters (measured according to ISO 3382 standard) will be discussed, also in respect of the initial reaction from ochestral musicians.

$6: 20$

1pAAb16. Qualitative Standard for Symphonic Concert Environment. Christopher Jaffe (167 East Rocks Road, Norwalk, CT 06851, USA, ADK117@GMAIL.COM)

All acousticians strive to obtain a symphonic concert environment that will be well received by musicians, professional music critics and the general public. Currently, this environment corresponds to the reflective energy measurements found in what might be designated as the rectangular European or American Concert Hall of the late nineteenth century, such as the Grosser Musikvereinssaal in Vienna and Symphony Hall in Boston Massachusetts. It is well known that these measurements can be correlated to subjective musical judgments and that halls incorporating these measurements have been universally designated as having excellent environments for symphonic concerts. But how did these specific reflections become an acoustical standard. This paper presents a theory of how the rectangular European and American style concert hall became an acoustical standard at the beginning of the twentieth century and poses the question as to whether this standard is still applicable in the twenty first century? 


\section{6:40}

1pAAb17. Acoustical characterization of orchestra platforms. Maria Giovannini (Politecnico di Torino, Corso Duca degli Abruzzi, 24, 10129 Turin, Italy, maria.giovannini@polito.it), Arianna Astolfi (Politecnico di Torino, Department of Energetics, Corso Duca degli Abruzzi, 24, 10129 Turin, Italy, arianna.astolfi@polito.it), Giulio Barbato (Politecnico di Torino, Corso Duca degli Abruzzi, 24, 10129 Turin, Italy, giulio.barbato @ polito.it)

In concert halls and other performance spaces it is important that acoustic conditions allow the musicians to hear each other and that there is a sufficient response from the room. Acoustical characterization of spaces for musicians, such as orchestra platforms, orchestra pits, and rehearsal rooms requires the measurements of the Early and Late Support, and other acoustical parameters described in the ISO/DC 3382-1 and in Literature. This work shows results from measurements carried out on five orchestra platforms, different in shape, size and materials of the boundary surfaces. Up to eight source positions were chosen in order to represent the main instrumental sections of the orchestra playing on the platform. Measurements were carried out without musicians, in conditions of empty stage and stage equipped as in concert configuration with stands, chairs, and risers. Some metrological problems connected with the reproducibility of results are considered as the directivity of the source and the small movements of the source and the microphone. The correlations between objective data show that the measured parameters are not always independent, but some groups of correlated measures, not completely separated, are found. The comparison of results allows to draw some connections with the main stage architectural features.

\section{7:00}

1pAAb18. On the Reverberation time of Gamelan Bali Concert Hall based on the Physio-Acoustic Responses. I Gde Nyoman Merthayasa (Institute of Technology Bandung, Dept. of Engineering Physics - ITB, Jln. Ganesha no. 10, 40132 Bandung, Indonesia, ignmerth@tf.itb .ac.id), Farida Idealistina Muchtadi (Institute of Technology Bandung, Dept. of Engineering Physics - ITB, Jln. Ganesha no. 10, 40132 Bandung, Indonesia, lies@tf.itb.ac.id)

Based on the fourth orthogonal acoustic factors of a concert hall design theory, it is possible to calculate the acoustical quality at any position in a proposed Gamelan Bali concert hall. And, it is well known that the value of preferred sound fields depends upon the characteristics of the sound source signal. Previous research has reported the most preferred value of the four orthogonal factors of Gamelan Bali sound fields based on the subjective response. It would be important to obtain the objective response, based on the physio-acoustics measurement using Electroechepalograph (EEG), when the subjects were excited by a certain variation of sound fields while other parameters were kept constant. In this research, the subjects were excited by a serial variation of the subsequent reverberation time of Gamelan Bali music. The brain activities were measured using EEG in the temporal areas. The result shows that the maximum power spectral of Alpha Waves of the subjects occurred when the reverberation time of Gamelan Bali were between $750 \mathrm{mS}$ to $1750 \mathrm{mS}$.

\title{
Session 1pAAc
}

\section{Architectural Acoustics and Psychological and Physiological Acoustics: Speech Segregation in Rooms I}

\author{
Barbara Shinn-Cunningham, Cochair \\ Boston University Hearing Research Center, 677 Beacon Street, Boston, MA 02215, USA \\ Mathieu Lavandier, Cochair \\ Cardiff University, School of Psychology, Tower Building, Park Place, Cardiff, CF10 3AT, UK
}

\section{Invited Papers}

1:00

1pAAc1. Issues in the perception of multiple speech sources under reverberant listening conditions. Chris J. Darwin (University of Sussex, Psychology, School of Life Sciences, BN1 9QG Brighton, UK, cjd@ sussex.ac.uk)

This paper will review the changes that room acoustics can make to the cues available for speech perception and those available for the localisation of sound sources, and give an overview of the perceptual mechanisms underlying the causes of the reduced intelligibility that reverberant room acoustics can make to the intelligibility of speech that is masked by additional sources of speech. Major factors that contribute to the interference of one source of speech by another are the obscuring of primary speech cues either by masking or by the failure of simultaneous grouping mechanisms, and the failure to track the sounds of an individual talker across time. The role of spatial localisation mechanisms to both these factors will be discussed.

$1: 20$

1pAAc2. Room-acoustic factors in attentional tracking. Anthony J. Watkins (Reading University, Department of Psychology, RG6 6AL Reading, UK, syswatkn@rdg.ac.uk), Simon J. Makin (Reading University, Department of Psychology, RG6 6AL Reading, UK, S.J.Makin@reading.ac.uk), Andrew Raimond (Reading University, Department of Psychology, RG6 6AL Reading, UK, a.raimond@ reading.ac.uk)

In a "busy" auditory environment listeners can selectively attend to one of several simultaneous messages by tracking one listener's voice characteristics. Here we ask how well other cues compete for attention with such characteristics, using variations in the spatial position of sound sources in a (virtual) seminar room. Listeners decided which of two simultaneous target words belonged in an attended "context" phrase when it was played with a simultaneous "distracter" context that had a different wording. Talker difference was 
in competition with a position difference, so that the target-word chosen indicates which cue-type the listener was tracking. The main findings are that room-acoustic factors provide some tracking cues, whose salience increases with distance separation. This increase is more prominent in diotic conditions, indicating that these cues are largely monaural. The room-acoustic factors might therefore be the spectral- and temporal-envelope effects of reverberation on the timbre of speech. By contrast, the salience of cues associated with differences in sounds' bearings tends to decrease with distance, and these cues are more effective in dichotic conditions. In other conditions, where a distance and a bearing difference cooperate, they can completely override a talker difference at various distances.

\section{$1: 40$}

1pAAc3. Effects of reverberation on spatial release from masking. Richard L. Freyman (Univ. of Massachusetts, Dept. of Communication Disorders, 358 N. Pleasant St., Amherst, MA 01003, USA, rlf@ comdis.umass.edu), Patrick M. Zurek (Sensimetrics Corp, 48 Grove St., Somerville, MA 02144, USA, pat@sens.com)

When sounds that we want to listen to (targets) and interfering sounds (maskers) are separated spatially, several advantages are observed relative to the baseline condition in which target and masker come from the same location. This paper will discuss how and to what extent these advantages are disrupted by room reverberation, which reduces better-ear signal-to-noise ratios and interaural intensity differences, and obscures interaural time differences. Statistical analysis of room acoustics combined with a model of binaural release from masking can, with the articulation index, help quantify expected reductions in speech recognition benefits of target/masker spatial separation in reverberation. This paper will also discuss some advantages of target/masker spatial separation that appear to be well preserved in reverberant rooms. Specifically, reverberation does not eliminate the perceptual differences between target and masker that arise from their different physical locations. The advantages of perceived spatial differences are seen when target and masking sounds share similar characteristics and are confusable with one another. In such cases spatial differences appear to resolve the confusion. This paper will describe research indicating that barely discriminable spatial differences between target and masker are sufficient for spatial release from masking in these circumstances. [Work supported by NIH DC-01625]

\section{2:00}

1pAAc4. Some Temporal and Spatial Effects of Room Acoustics on Speech Privacy. John S. Bradley (National Research Council, 1200 Montreal Rd, Ottawa, ON K1A 0R6, Canada, john.bradley@nrc-cnrc.gc.ca), Marina Apfel (National Research Council, 1200 Montreal Rd, Ottawa, ON K1A 0R6, Canada, Marina.Apfel@gmx.de), Brad N. Gover (National Research Council, 1200 Montreal Rd, Ottawa, ON K1A 0R6, Canada, Brad.Gover@nrc-cnrc.gc.ca)

The speech privacy of meeting rooms relates to the possibility of eavesdroppers outside the room being able to understand speech from within the meeting room. Although the speech privacy of meeting rooms is usually assumed to relate to the level of the speech transmitted from the room relative to the ambient noise outside the room, the intelligibility of the transmitted speech is also influenced by room acoustics. However, the audibility of speech is only influenced by the levels of the transmitted speech and ambient noise. This paper reports on a series of speech intelligibility tests in which the components of the problem were systematically added. As expected, adding early reflections of speech from various directions increases intelligibility but later-arriving speech sounds decrease intelligibility. While a spatial separation of speech and noise sources in free field conditions increases speech intelligibility (spatial release from masking), in more realistic conditions, representing a diffuse ambient noise field, the effects are much smaller. Conditions are further complicated by the sever filtering of the speech on transmission through typical walls. The combined effects can be estimated and are significant. If ignored costly over-design of the sound isolation of the meeting room could occur.

\section{$2: 20$}

1pAAc5. Binaural room acoustics - cross-correlation. William M. Hartmann (Michigan State University, Dept. of Physics and Astronomy, East Lansing, MI 48824, USA, hartmann@pa.msu.edu), Neil L. Aaronson (Michigan State University, Dept. of Physics and Astronomy, East Lansing, MI 48824, USA, AARONSON@PA.MSU.EDU)

The subject of "Binaural room acoustics" combines the disciplines of room acoustics with facts and models of human binaural physiology and psychology. It considers the linear effects of rooms on the inputs to the two ears of a listener, and it considers the nonlinear effects of binaural hearing processes on perceptually relevant acoustical characterizations of rooms. Linear effects of interest are binaural differences in amplitude and phase spectra, which are combined in the cross-correlation function (CCF). A psychophysically relevant CCF is computed over frequency bands approximately corresponding to auditory filters. Because the bands are narrow, the effects on the CCF of frequency-dependent interaural parameters, most importantly dispersion, can be modeled analytically. The roles of amplitude incoherence and dispersion can also be independently modelled. A systematic experimental approach begins with the dependence of the CCF on head related transfer functions (HRTF) as measured at different azimuths in a free field. Although there are notable individual differences, the effects of HRTFs appear to be almost entirely below detection threshold. An empirical relationship between the CCF for a waveform and for its envelope has been established numerically and in extensive room experiments. [Work supported by the NIDCD, grant DC00181.]

\section{2:40}

1pAAc6. Speech segregation in rooms: Importance of the interferer interaural coherence. Mathieu Lavandier (Cardiff University, School of Psychology, Tower Building, Park Place, CF10 3AT Cardiff, UK, lavandiermn@ cardiff.ac.uk), John F. Culling (Cardiff University, School of Psychology, Tower Building, Park Place, CF10 3AT Cardiff, UK, CullingJ@ cardiff.ac.uk)

When an interferer is present, reverberation degrades speech intelligibility not only by its direct effect on the target, but also by affecting the interferer [Lavandier \& Culling, J. Acoust. Soc. Am., 122 (3), 2007]. This latter effect occurs at lower levels of reverberation than the former intrinsic degradation of the target, and can be explained by a binaural mechanism. We show here that the interaural coherence of the interferer is the principal parameter predicting the resulting loss of intelligibility. Speech reception thresholds were measured under headphones, using running speech targets and speech-shaped noise interferers. The stimuli were created by con- 
sidering a listener and a spatially-separated target and interferer in virtual rooms. The reverberation was varied by modifying the absorption coefficient of every room surfaces simultaneously. Different absorption coefficients were used for target and interferer. With the target anechoic, the interferer was tested in several configurations, in rooms with different sizes and absorptions, at different distances and azimuths from the listener. Several computations of coherence were compared, in order to reveal the one allowing the best prediction of intelligibility.

\section{3:00}

1pAAc7. Effects of pitch and spatial separation on selective attention in anechoic and reverberant environments. Scott Bressler (Boston University Hearing Research Center, 677 Beacon Street, Boston, MA 02215, USA, SCBressler@verizon.net), Barbara Shinn-Cunningham (Boston University Hearing Research Center, 677 Beacon Street, Boston, MA 02215, USA, shinn@cns.bu.edu)

Subjects identified a random, spoken sequence of five monotonized digits $\left(\mathrm{F}_{0}=100 \mathrm{~Hz}\right)$ presented from $0^{\circ}$ azimuth. A monotonized masking sentence $\left(\mathrm{F}_{0}=84,89,94,100,106,112\right.$, or $\left.119 \mathrm{~Hz}\right)$ was presented simultaneously from either $0^{\circ}$ and $+90^{\circ}$ azimuth (chosen randomly on each trial). The same talker recorded both target and masker speech. KEMAR-derived transfer functions simulated either a reverberant or anechoic environment. In contrast to previous studies, in the anechoic condition, differences in pitch provided little benefit and differences in location gave improvements explainable by improvements in the target-to- masker ratio at the acoustically better ear. In reverberant conditions, differences in target and masker location improved performance more than differences in pitch; however, performance was best when there were differences in both location and pitch. Results suggest that when a target utterance is easy to segregate and select (such as in anechoic space when the target is a digit sequence embedded in a competing sentence), highlevel attributes such as pitch and location do not improve the ability to selectively attend in a speech-on-speech task. However, in more challenging, reverberant conditions, location and pitch cues can aid segregation and/or selection.

1pAAc8. Effects of reverberation on perceptual segregation of competing voices by difference in fundamental frequency. Mickael Deroche (Cardiff University, School of Psychology, Tower Building, Park Place, CF10 3AT Cardiff, UK, derochem@cf.ac.uk), John F. Culling (Cardiff University, School of Psychology, Tower Building, Park Place, CF10 3AT Cardiff, UK, CullingJ@cardiff.ac.uk)

Three experiments investigated the effect of reverberation on listeners' ability to use the difference in fundamental frequency (F0) between a target and an interfering voice to perceptually separate them. Speech reception thresholds (SRTs) were measured for a monotonized or frequency-modulated male voice against one or two other monotonized or frequency-modulated male voices or against a speech-shaped, pulse train within a virtual room with controlled reverberation. The sources of targets and interferers were always co-located both straight ahead of the listener. In the two first experiments, the beneficial effect of a F0 difference was reduced in reverberation when the target and interferer's F0 were frequency-modulated at $5 \mathrm{~Hz}$. Experiment 3 investigated this interaction between reverberation, a F0 difference and the target and/or interferer F0's modulation: the results highlighted the relevance of the interferer F0's modulation alone in the presence of reverberation. This finding is consistent with the idea that the effect of F0 difference is mediated by a harmonic-cancellation process. The cancellation of the interferer is disrupted by the reverberation only when the interferer's F0 is frequency-modulated, irrespective of the modulation of the target's F0. A frequency-overlap between the F0 of the target and the maskers does not seem a cause of worse impairment in intelligibility.

\section{3:40-4:00 Break}

\section{4:00}

1pAAc9. The effects of fluctuating interaural cues on the segregation of speech in rooms: Revisited. Douglas S. Brungart (AFRL, 2610 Seventh Street, WPAFB, OH 45433, USA, douglas.brungart@ wpafb.af.mil), Nandini Iyer (AFRL, 2610 Seventh Street, WPAFB, OH 45433, USA, nandini.iyer@wpafb.af.mil)

Spatial separation is known to improve the segregation of talkers in anechoic environments, but spatial auditory cues can be severely degraded in reverberant rooms. One might expect the random disruptions in the interaural time and level differences (ILDs and ITDs) that occur in reverberant environments to eliminate many of the intelligibility benefits that normally occur for spatially separated speech. However, the precedence effect often leads to a robust perception of spatial location even in extremely echoic environments. This can result in an apparent separation between talkers that may facilitate selective attention to the location of the target speech even in a highly reverberant room. Also, random fluctuations in ITD and ILD may lead to differences in the apparent source widths of the target and masking sounds, which might serve as a segregation cue. In this talk, we examine the effects fluctuating interaural difference cues have on the segregation of target speech from competing speech or noise. These results, originally scheduled for presentation at the 152nd meeting, suggest that differences in apparent source width can be used to segregate competing speech signals even when the target and masking signals appear to originate from the same direction relative to the listener.

\section{4:20}

1pAAc10. A reverberation-robust automatic speech recognition system based on temporal masking. Guy J. Brown (University of Sheffield, Dept. of Computer Science, Regent Court, 211 Portobello Street, S1 4DP Sheffield, UK, g.brown@dcs.shef.ac.uk), Kalle J. Palomäki (Adaptive Informatics Research Centre, Helsinki University of Technology, P.O. Box 5400, 02015 Espoo, Finland, kalle .palomaki@tkk.fi)

Previously, we have proposed a reverberation-robust system for automatic speech recognition (ASR) based on a temporal masking principle. In the first pathway of this system, speech is analysed by a bank of auditory filters, in order to provide acoustic features for the recogniser. In the second pathway, a bandpass modulation filter $(1.5 \mathrm{~Hz}-8.2 \mathrm{~Hz})$ detects regions of the envelope in each filter 
channel that contain strong speech energy. Regions of the modulation filter output that exceed a threshold are labelled as reliable evidence for the speech in a time-frequency mask; regions that fall below the threshold are dominated by reverberation and labelled as unreliable. The acoustic features and time-frequency mask are then decoded by a "missing data" ASR system. Here we describe modifications of this system that bring it into closer agreement with purported mechanisms of human perceptual compensation for reverberation, as determined by psychophysical studies [Watkins \& Makin, JASA 121, 257-266]. Specifically, we use a metric for identifying regions of reliable speech that gives greater emphasis to reverberation "tails," and is computed independently for each frequency band. The performance of the system is evaluated on a corpus of spoken digits convolved with recorded room impulse responses.

\title{
4:40
}

1pAAc11. Monaural segregation of reverberant speech. Zhaozhang Jin (Ohio State University, Department of Computer Science \& Engineering, 2015 Neil Ave., Columbus, OH 43210, USA, jinzh@cse.ohio-state.edu), Deliang Wang (Ohio State University, Department of Computer Science \& Engineering, 2015 Neil Ave., Columbus, OH 43210, USA, dwang@cse.ohio-state.edu)

A major source of signal degradation in realistic environments is room reverberation. Monaural speech segregation in reverberant environments is a particularly challenging problem. Although inverse filtering has been proposed to partially restore the harmonicity of reverberant speech before segregation, this approach is sensitive to different room configurations. In this study, we investigate monaural segregation of reverberant speech by employing a supervised learning approach to map from a set of pitch-based auditory features to a grouping cue, which encodes the posterior probability of a time-frequency unit being target dominant given observed features. We devise a novel objective function for the learning process, which relates to the goal of maximizing SNR directly. The models trained using this new objective function yield significantly better results for unit labeling. In our segregation system, a segmentation and grouping framework is utilized in order to capture segments reliably under reverberant conditions and organize them into streams. Systematic evaluations show that our approach produces very promising results.

MONDAY AFTERNOON, 30 JUNE 2008

ROOM 202/203, 4:40 TO 7:20 P.M.

\section{Session 1pAAd}

\section{Architectural Acoustics and Musical Acoustics: Surround Sound Acoustics I}

\author{
Anthony Hoover, Cochair \\ McKay Conant Hoover Inc., 5655 Lindero Canyon Rd, Suite 325, Westlake Village, CA 91362, USA \\ Alexander Case, Cochair \\ Fermata Audio \& Acoustics, PO Box 1161, Portsmouth, NH 03802, USA
}

Etienne Corteel, Cochair

Sonic Emotion, Eichweg, 6, Oberglatt, CH-8154, Switzerland

\section{Contributed Papers}

\section{4:40}

1pAAd1. Virtual acoustic environments for music performance, rehearsal, and recording. Wieslaw Woszczyk (McGill University, Schulich School of Music, 555 Sherbrooke Street West, Montreal, QC H3A 1E3, Canada,wieslaw@music.mcgill.ca), Doyuen Ko (McGill University, Schulich School of Music, 555 Sherbrooke Street West, Montreal, QC H3A 1E3, Canada, doyuen.ko@mail.mcgill.ca),Tom Beghin (McGill University, Schulich School of Music, 555 Sherbrooke Street West, Montreal, QC H3A 1E3, Canada, tom.beghin@mcgill.ca), Martha De Francisco (McGill University, Schulich School of Music, 555 Sherbrooke Street West, Montreal, QC H3A 1E3, Canada, martha.defrancisco@mcgill.ca), Jeremy Tusz (McGill University, Schulich School of Music, 555 Sherbrooke Street West, Montreal, QC H3A 1E3, Canada, tusz@mac.com), Ryan Miller (McGill University, Schulich School of Music, 555 Sherbrooke Street West, Montreal, QC H3A 1E3, Canada, ryan.miller@mail.mcgill.ca)

A comprehensive 24-channel sound field rendering system was built at McGill University's CIRMMT Centre to immerse scholar/performer Tom Beghin in virtual concert spaces where Haydn composed and performed his keyboard sonatas, or expected them to be performed. The virtual rooms were reconstructed from detailed measurements made in Haydn's historical rooms in Europe using a high-resolution $24 \mathrm{bit} / 96 \mathrm{kHz}$ impulse-response measuring system. In an acoustically treated laboratory, 24 custom loudspeaker arrays were arranged on a surface of a hemisphere surrounding the performer. Multiple fast DSP engines convolved live signals with 24 im- pulse responses allowing the performer to rehearse and record in each room with low-latency virtual acoustics. This paper describes the details of system design, the method of measurement, and discusses various aspects of recording and performing in virtual acoustic environments. The method has also been tested in a large room during public performance by having virtualroom responses and high-definition photo images projected from the stage. Surround sound recordings of Haydn's complete solo keyboard music made in several virtual rooms and matched to specific historical instruments will be released in a collection of 13 commercial SA-CD's by the end of 2008 .

\section{5:00}

1pAAd2. Prediction of spatial perceptual attributes of reproduced sound across the listening area. Philip J. Jackson (University of Surrey, Centre for Vision, Speech and Signal Processing, GU2 7XH Guildford, UK, p.jackson@surrey.ac.uk), Martin Dewhirst (University of Surrey, Institute of Sound Recording, GU2 7XH Guildford, UK, m.dewhirst@surrey.ac.uk), Slawomir Zielinski (University of Surrey, Institute of Sound Recording, GU2 7XH Guildford, UK, s.zielinski@surrey.ac.uk), Francis J. Rumsey (University of Surrey, Institute of Sound Recording, GU2 7XH Guildford, UK, f.rumsey@surrey.ac.uk), Robert Conetta (University of Surrey, Institute of 
Sound Recording, GU2 7XH Guildford, UK, r.conetta@surrey.ac.uk),David Meares (DJM Consultancy, Winthrift, Nuthurst, RH13 6RG Horsham, UK, meares_david@yahoo.co.uk), Søren Bech (Bang \& Olufsen A/S, Peter Bangsvej 15, DK-7600 Struer, Denmark, sbe@bang-olufsen.dk)

Audio systems and recordings are optimized for listening at the "sweet spot," but how well do they work elsewhere? An acoustic-perceptual model has been developed that simulates sound reproduction in a variety of formats, including mono, two-channel stereo, five-channel surround and wave- field synthesis. A virtual listener placed anywhere in the listening area is used to extract binaural signals, and hence interaural cues to the spatial attributes of the soundfield. Using subjectively-validated models of spatial sound perception, we can predict the way that human listeners would perceive these attributes, such as the direction (azimuth) and width of a phantom source. Results will be presented across the listening area for different source signals, sound scenes and reproduction systems, illustrating their spatial fidelity in perceptual terms. Future work investigates the effects of typical reproduction degradations.

\section{Invited Papers}

1pAAd3. Measuring perceived spatial quality changes in surround sound reproduction. Francis J. Rumsey (University of Surrey, Institute of Sound Recording, GU2 7XH Guildford, UK, f.rumsey@surrey.ac.uk), Slawomir Zielinski (University of Surrey, Institute of Sound Recording, GU2 7XH Guildford, UK, s.zielinski@surrey.ac.uk), Philip J. Jackson (University of Surrey, Centre for Vision, Speech and Signal Processing, GU2 7XH Guildford, UK, p.jackson@surrey.ac.uk), Martin Dewhirst (University of Surrey, Institute of Sound Recording, GU2 7XH Guildford, UK, m.dewhirst@surrey.ac.uk), Robert Conetta (University of Surrey, Institute of Sound Recording, GU2 7XH Guildford, UK, r.conetta@surrey.ac.uk), Søren Bech (Bang \& Olufsen A/S, Peter Bangsvej 15, DK-7600 Struer, Denmark, sbe@bang-olufsen.dk), David Meares (DJM Consultancy, Winthrift, Nuthurst, RH13 6RG Horsham, UK, meares _david@yahoo.co.uk)

The spatial quality of audio content delivery systems is becoming increasingly important as service providers attempt to deliver enhanced experiences of spatial immersion and naturalness in audio-visual applications. Examples are virtual reality, telepresence, home cinema, games and communications products. The QESTRAL project is developing an artificial listener that will compare the perceived quality of a spatial audio reproduction to a reference reproduction. The model is calibrated using data from listening tests, and utilises a range of metrics to predict the resulting spatial sound quality ratings. Potential application areas for the model are outlined, together with exemplary results obtained from some of its component parts.

1pAAd4. Dynamic sound image creation by un-directional sound beam reproduction toward an interior surface with a DSP controlled line array speaker for Surround Sound Composition. Yasushi Shimizu (Center for Advanced Sound Technologies, Yamaha, 203 Matsunokijima, 4380192 Iwata, Shizuoka, Japan, yasushi_shimizu@gmx.yamaha.com), Masato Hata (Yamaha, 203 Matsunokijima, 4380192 Iwata, Shizuoka, Japan, hata@beat.yamaha.co.jp), Atsuko Ito (Center for Advanced Sound Technologies, Yamaha, 203 Matsunokijima, 4380192 Iwata, Shizuoka, Japan, atsuko1_ito@gmx.yamaha.com), Akira Miki (Yamaha, 203 Matsunokijima, 4380192 Iwata, Shizuoka, Japan, akira_miki@gmx.yamaha.com), Masato Hatanaka (Hashimoto Bldg, 301, 4-1-25, Komazawa, Setagaya, 1540012 Tokyo, Japan, info@ hatanakamasato.net)

Surround Sound Composition in an indoor space is frequently provided with spatial sounds, giving both a sound localization and surround sound effect. Systems have been installed in public spaces such as a museum and retail shops. However, technical improvements are required to provide a small sweet spot and to address architectural designs for speaker installation, because multiple speakers are required for surround sound effect. Recently, we have been applying DSP beam-forming technology with a line-array speaker to create a 3D sound image by scanning a focused sound beam onto an interior surface. The DSP array speaker is capable of providing static and dynamic control in beam steering and sound focusing. Therefore, dynamic spatial sound effect on the interior surface is possible, wherein un-directional sound reproduction is analogous to un-directional lighting. We present three case studies; 1) Virtual 3D Surround Sound in multi-media improvisation, 2) 3D Spatial Sound Synthesis in sound installation and 3) Sound Installation with a Dynamic Sound Beam Scanning.

\section{6:00}

1pAAd5. How does spatial auditory perception impact how we enjoy surround sound? Barbara Shinn-Cunningham (Boston University Hearing Research Center, 677 Beacon Street, Boston, MA 02215, USA, shinn@cns.bu.edu), K Anthony Hoover (McKay Conant Hoover, Inc., 5655 Lindero Canyon Road, Suite 325, Westlake Village, CA 91362, USA, thoover@mchinc.com)

Surround sound has become an essential element of enormously popular home entertainment systems, with consumers becoming increasingly eager to invest in high-quality audio systems. While even a cursory web search can uncover numerous criteria, standards, and blogs describing how to select and set up surround-audio equipment, architectural effects are typically ignored or, at best, treated naively. However, architectural acoustics profoundly affect the perceptual experience of a surround-sound listener. Similarly, sound engineers, producers, and artists agonize over every aspect of soundtrack design and production, including the spatial cues embedded in a surround-sound recording, but they have no control over the acoustic environments in which consumers experience their art. How do the acoustics of surround-listening spaces influence the perceptual experiences of the ordinary listener? Is accurate sound localization a proper, desirable, or achievable goal? Would more loudspeakers or higher fidelity improve the surround experience of a typical consumer? These issues will be discussed, taking into account basic psychophysical issues as well as disheartening truths about how ordinary consumers experience sound from their expensive home entertainment systems. 
1pAAd6. Acoustics of a large immersive environment, The Allosphere. David Conant (McKay Conant Hoover, Inc., 5655 Lindero Canyon Road, Suite 325, Westlake Village, CA 91362, USA, dconant@mchinc.com), William Chu (McKay Conant Hoover, Inc., 5655 Lindero Canyon Road, Suite 325, Westlake Village, CA 91362, USA, wchu@mchinc.com), Thomas McNally (McKay Conant Hoover, Inc., 5655 Lindero Canyon Road, Suite 325, Westlake Village, CA 91362, USA, tmcnally@ mchinc.com), K Anthony Hoover (McKay Conant Hoover, Inc., 5655 Lindero Canyon Road, Suite 325, Westlake Village, CA 91362, USA, thoover@mchinc.com), Stephen Pope (Center for Research in Electronic Art Technology, U. C. Santa Barbara, 220 Santa Anita Road, Santa Barbara, CA 93105, USA, Stephen@HeavenEverywhere.com)

The Allosphere is a new audio/visual immersion space for the California Nanosystems Institute at The University of California, Santa Barbara, used for both scientific and performing-arts studies. This three-story sphere with central-axis catwalk permits an unusually large experiential region. The huge perforated-metal visual-projection sphere, with its principle listening locations centered inside the sphere, introduces multiple considerations and compromises, especially since the ideal acoustical environment is anechoic. Video projection requires opaque light reflectivity of the concave projection surface, while audio solicits extreme sound transmissibility of the screen plus full-range sound absorptivity outside the sphere. The design requires high-fidelity spatialization of a large number of simulated sound sources over a large region near the core, and support of vector-based amplitude panning, Ambisonic playback, and wavefield synthesis. This paper discusses considerations that both conform to, and lie outside of, traditional acoustical analysis methodologies, and briefly reviews the electroacoustic systems design.

\section{6:40}

1pAAd7. Case study on assessing audibility by "Perspicuity" for sounds added to factory noise, using different types of speaker in directional performance. Yasushi Shimizu (Center for Advanced Sound Technologies, Yamaha, 203 Matsunokijima, 4380192 Iwata, Shizuoka, Japan, yasushi_shimizu@gmx.yamaha.com), Mai Fujiwara (Center for Advanced Sound Technologies, Yamaha, 203 Matsunokijima, 4380192 Iwata, Shizuoka, Japan, mai_fujiwara@gmx.yamaha.com), Atsuko Ito (Center for Advanced Sound Technologies, Yamaha, 203 Matsunokijima, 4380192 Iwata, Shizuoka, Japan, atsuko1_ito@gmx.yamaha.com)

Music and other sounds have been played to provide a pleasant sound environment for workers in a factory. In terms of "Sound Perspicuity" this case study was conducted to assess audibility of spatial sounds, which were adjusted in level without increasing total sound pressure level (SPL) at the workstation. Furthermore, the added sounds were not audible at other workstations. Three speaker systems, including line-array speaker $(60 \mathrm{~cm}$ in length), were used for the case study. The ratio of added sound (Signal) to factory noise (Noise) was analyzed at every measuring point on a grid of $50 \mathrm{~cm}$ by $50 \mathrm{~cm}$. Audibility listening tests were performed for the added sounds, such as natural-environment sounds and musical instrument tones, with Signal to Noise ratios analyzed from three types of speaker. As a result, a sound is recognized as "Kiwadachi" by a Japanese adjective word even when the factory noise has higher levels than the added sounds. Audibility test results for each added sound are discussed with different Signal to Noise ratios.

\section{Contributed Paper}

\section{7:00}

1pAAd8. Spatial Audio Reproduction Using Distributed Mode Loudspeakers. Joshua Atkins (Johns Hopkins University, 853 West 35th Street, Baltimore, MD 21211, USA, joshatkins@jhu.edu), James West (Johns Hopkins University, Department of Electrical Engineering, 3400 North Charles Street, Baltimore, MD 21218, USA, jimwest@jhu.edu)

For future telecommunication systems to become more pervasive in society they must have the ability to produce high quality surround sound audio for multiple listeners. Current 5.1 audio systems lack the capacity to handle large group telecommunications because of their limited optimal listening positions ("sweet spots") and their preference for sound generated in front of the listeners. This is due in part to the non-optimal position of the loudspeakers and also to the fact that very directional speakers are commonly used. Recently, a new form of speaker has been developed called a distributed mode loudspeaker (DML). These speakers use bending waves in a panel to radiate sound. Consequently, they can be very good omnidirectional sources throughout nearly all of the human hearing range. Experiments using a higher order ambisonics (HOA) approach to sound field capture and generation will be presented to show how conventional electrodynamic speaker arrays compare to equivalent DML arrays for spatial sound reproduction. This work is funded by NSF Grant IIS-0534221. 


\title{
Session 1pAAe
}

\section{Architectural Acoustics and Noise: Low Frequency Absorption: Mechanisms, Measurement Methods, and Application I}

\author{
Peter D'Antonio, Cochair \\ RPG Diffusor Systems, Inc., 651-C Commerce Drive, Upper Marlboro, MD 20774, USA \\ Christian Nocke, Cochair \\ Akustikbüro Oldenburg, Katharinenstr. 10, Oldenburg, 26121, Germany
}

Invited Papers

5:00

1pAAe1. Overview of low frequency control options in rooms. Peter D'Antonio (RPG Diffusor Systems, Inc., 651-C Commerce Drive, Upper Marlboro, MD 20774, USA, pdantonio@rpginc.com)

Low frequency control of modes and speaker boundary interference in rooms has been a persistent problem that affects the sound field in rooms. However, many innovative techniques have been developed to address these problems. This presentation will be an overview of approaches that address the optimization of room dimensions to provide the flattest room response and speaker/listener placement to minimize speaker-boundary interference; the mechanism and design of passive absorption devices based on Helmholtz resonators, diaphragmatic limp mass membranes, plate resonators and microperforated panels will be described and characterized; as well as electro-acoustic approaches, using equalization, active absorbers and the optimal placement of multiple in-phase subwoofers.

$5: 20$

1pAAe2. Low frequency sound absorption measurements - limits and ideas. Christian Nocke (Akustikbüro Oldenburg, Katharinenstr. 10, 26121 Oldenburg, Germany, nocke@akustikbuero-oldenburg.de)

Many (standardised) measurement procedures for sound absorption rely on geometrical approximations or statistical approaches. They fail whenever the limit of geometrical acoustics is reached. Also many room acoustic modeling procedures assume geometrical spreading of sound and neglect the wave characteristics of sound waves. In this paper various approaches towards the measurement of sound absorption are briefly reviewed and discussed in the light of low frequency applications. Approaches towards analytic descriptions for sound fields in room at low frequencies from the early 1930s are reviewed. The approaches might be the basis for future developments on new procedures for measurement techniques. Finally measurement results on the deduction of sound absorption at low frequencies are presented.

\section{$5: 40$}

1pAAe3. Enhancing bass absorbers using activated carbon. Fouad Bechwati (University of Salford, Acoustics Research Centre, Newton Building, M5 4WT Salford, UK, f.bechwati@pgr.salford.ac.uk), Trevor J. Cox (University of Salford, Acoustics Research Centre, Newton Building, M5 4WT Salford, UK, t.j.cox@salford.ac.uk), Olga Umnova (University of Salford, Acoustics Research Centre, Newton Building, M5 4WT Salford, UK, o.umnova@salford.ac.uk), Mark R. Avis (University of Salford, Acoustics Research Centre, Newton Building, M5 4WT Salford, UK, m.r.avis@salford.ac.uk)

Bass absorbers, either Helmholtz or membrane devices, are commonly used in small rooms to reduce the effects of standing wave modes. Previously, it has been shown that activated carbon can be used to change the compliance of loudspeaker enclosures. It has also been shown that activated carbon displays excess absorption at low frequencies. It has been suggested that the change in compliance and absorption is due to adsorption and desorption of air molecules on the surface of the carbon, caused by the compressions and rarefactions of sound waves. This paper investigates the extent of these effects to and their significance for resonant absorbers. The results show that activated carbon can lower the resonant frequency of a Helmholtz absorber, and also increases the quality factor of the resonance. The paper will discuss what the results mean in terms of the physical interaction between activated carbon and sound, as well as the practical application of the material to bass absorbers.

\section{6:00}

1pAAe4. An improved low frequency radiation model for finite sound reflectors. Jonathan Rathsam (Univ. of Nebraska - Lincoln, Architectural Eng. Program, 1110 S. 67th St., Omaha, NE 68182-0681, USA, jrathsam@mail.unomaha.edu), Lily M. Wang (University of Nebraska - Lincoln, 1110 S. 67th St., Omaha, NE 68182-0681, USA, LWang4@UNL.edu), Jens Holger Rindel (Odeon A/S, Scion DTU, Diplomvej Buildling 381, DK-2800 Lyngby, Denmark, jhr@odeon.dk), Claus Lynge Christensen (Odeon A/S, Scion DTU, Diplomvej Buildling 381, DK-2800 Lyngby, Denmark, clc@odeon.dk)

Geometric computer models for room acoustics, such as ODEON, predict sound fields most reliably at high frequencies. At low frequencies, algorithms must be modified to account for deviations from geometrical acoustics caused by wave phenomena. For finite reflectors, a common low frequency model is based on the Kirchhoff-Fresnel Diffraction Approximation, which predicts a uniform $6 \mathrm{~dB}$ 
per octave slope below a reflector's geometrical limiting frequency. As discussed in this paper, highly accurate Boundary Element Method simulations, not subject to the Kirchhoff Approximation, suggest the use of an additional, lower limiting frequency and slope of $12 \mathrm{~dB}$ per octave to represent the reflector's response at the lowest frequencies. This second limiting frequency and $12 \mathrm{~dB}$ per octave slope, referred to as the dipole limiting frequency, are presented in a form suitable for insertion into a geometric computer model. [Work supported by the National Science Foundation.]

\section{Contributed Papers}

\section{6:20}

1pAAe5. Control of low-frequency sound to improve spatial uniformity in music production spaces. Ronald Eligator (Acoustic Dimensions, 145 Huguenot Street, New Rochelle, NY 10801, USA, religator @acousticdimensions.com)

Commercial music post-production spaces are often less than $300 \mathrm{ft}^{2}(28$ $\left.\mathrm{m}^{2}\right)$ and $2700 \mathrm{ft}^{3}\left(76.5 \mathrm{~m}^{3}\right)$, and it is not uncommon for them to be one-third to one-half this area. Even with careful room design, creating spatiallyuniform sound fields, especially at low-frequencies, can be thwarted by the physical restraints imposed by the room's dimensions, boundary conditions and restraints imposed by other functional requirements and budget. This paper presents successful, and not so successful, approaches to managing these challenges through speaker selection, placement and mounting, as well as room shaping and finishes and furniture design. Results of design analysis, room acoustic testing, and listening tests are compared.

\section{6:40}

1pAAe6. The Perforated Panel Resonator with Flexible Tube Bundles and its Appliations. Yadong Lu (Institute of Acoustics, Chinese Academy of Sciences, P. O. Box 2712, 21 Beisihuanxilu St., 100080 Beijing, China, yadong@mail.ioa.ac.cn), Huide Tang (Tianjin Hearing-aid Factory, Chenglinzhuang Rd. No. 93, 300161 Tianjin, China, tanghuide@eyou.com), Qiang Wang (Jinyusun Ventilation and Airconditioning Equipment Ltd., Golden-delta Development Region. Tongzhou Dist., 101112 Beijing, China, jinyushun2003@yahoo.com.cn), Jing Tian (Institute of Acoustics, Chinese Academy of Sciences, P. O. Box 2712, 21 Beisihuanxilu St., 100080 Beijing, China, tian@mail.ioa.ac.cn), Youli Qu (Beijing Electrical Designing Institute, 15 Guananmenxijie St., 100055 Beijing, China, thgink@tom.com), Bo Qiu (Institute of Acoustics, Chinese Academy of Sciences, P. O. Box 2712, 21 Beisihuanxilu St., 100080 Beijing, China, nkcubert@yahoo.com.cn)

The sound absorptive mechanism and properties of the perforated panel resonator with flexible tube bundles are presented. Based upon that, some practical applications of the sound absorbing structure are introduced as follows. First of all, the perforated panel resonator with flexible tube bundles is used in designing a highly sound absorptive chamber, which is used as an audio-video laboratory. The acoustical performances of the chamber are presented. Second, the perforated panel resonator with flexible tube bundles is used in highly efficient duct silencer. The total noise level of axial fan source is attenuated to background noise level. Not only middle and high frequency noise is reduced, but also low frequency noise is suppressed considerably. Third, the perforated panel resonator with flexible tube bundles is used in a small-type centrifugal fan's casing treatments. The cutoff region of the centrifugal fan's casing is also acoustically treated. After the acoustical treatments, total sound power level of the fan is reduced obviously. Fourth, the perforated panel resonator with flexible tube bundles is used in designing the exhaust ducts and sound absorptive channel walls for an electrical transformer substation. The noise emitted from the transformer substation is reduced to surrounding background noise level.

\section{7:00}

1pAAe7. Shape-optimization of several multi-modal resonators accounting for room/resonator acoustical coupling. Octávio Inácio (Musical Acoustics Laboratory - ESMAE-IPP, Rua da Alegria, 503, 4000-045 Porto, Portugal, OctavioInacio@esmae-ipp.pt), José Antunes (Applied Dynamics Laboratory, Instituto Tecnológico e Nuclear, Estrada Nacional 10, 2686-953 Sacavém, Portugal, jantunes@itn.pt)

Helmholtz resonators are often applied for the sound equalisation of control rooms, through adequate leveling of the low frequency acoustic modal room responses. In several recent papers we proposed to improve the efficiency of such devices by, instead of using basic Helmholtz resonators with uniform cross-section, develop shape optimized multi-modal resonators in order to cope with a larger number of intrusive room modes. We thus showed the feasibility of resonator shape-optimization, in order to obtain a target set of acoustic eigenvalues, within imposed physical and/or geometrical constraints. More recently, we developed an efficient substructure theoretical approach to compute the coupled acoustical modes of rooms fitted with several multi-mode resonators, later also including viscous boundary layer absorption effects at the room/resonator interfaces. In the present paper we extend a further step these results by applying the previously developed optimization techniques to the fully coupled room/resonator model. We thus obtain truly representative results for the optimized complex acoustical problem, which highlight the potential of the proposed corrective methodology. 


\title{
Session 1pAB
}

\section{Animal Bioacoustics, Noise, and ECUA: Anthropogenic Noise Effects on Animals I}

\author{
Michael Stocker, Cochair \\ Ocean Conservation Research, P.O. Box 559, Lagunitas, CA 94938, USA \\ Jonathan Gordon, Cochair \\ Sea Mammal Research Unit, Gatty Marine Laboratory, University of St Andrews, St Andrews, KY16 8LB, UK
}

\section{Contributed Papers}

\begin{abstract}
1:00
1pAB1. Automated classification of odontocetes in open ocean environments using a novel multiclass suport vector machine. Susan Jarvis (Worcester Polytechnic Institute, 100 Institute Rd, Worcester, MA 01609, USA, sjarvis@ece.wpi.edu), Nancy A. Dimarzio (Naval Undersea Warfare Center Division Newport, 1176 Howell Street, Bldg 1351, 2nd Floor, Newport, RI 02841, USA, dimarziona@npt.nuwc.navy.mil), Ronald P. Morrissey (Naval Undersea Warfare Center Division Newport, 1176 Howell Street, Bldg 1351, 2nd Floor, Newport, RI 02841, USA, morrisseyrp@npt.nuwc.navy.mil), Jessica Ward (NAVSEA, Newport Undersea Warfare Center, Newport, RI RI 02841, USA, wardja@npt.nuwc.navy.mil), David Moretti (NAVSEA, Newport Undersea Warfare Center, Newport, RI RI 02841, USA, MorettiDJ@npt.nuwc.navy .mil)
\end{abstract}

Mid-frequency active sonar has recently been implicated in several marine mammal stranding events in various geographic locations. This has caused increased interest in monitoring and mitigating the effects of anthropogenic noise on marine mammals. Some species can be more susceptible sonar than others. For example, beaked whales (particularly Mesoplodon densirostris) have been the predominant species involved in most of the strandings. Key to effective monitoring and mitigation is the ability to automatically detect and identify the animals present, especially the species of high interest like beaked whales. This paper presents a novel class-specific support vector machine (CS-SVM) methodology for automated, specieslevel classification of small odontocetes. A CS-SVM has been developed and trained to identify click vocalization from several species of odontocetes including Mesoplodon densirostris. The algorithm processes time series data in a fully automated fashion first detecting and then classifying click vocalizations. The current CS-SVM configuration correctly identifies clicks from M. densirostris over $95 \%$ of time. Further results from the application of this automated classifier to data recorded at the AUTEC and SCORE undersea range facilities will be presented.

\section{$1: 20$}

1pAB2. Effect of vessels and swimmers on the behavior of spinner dolphins (Stenella longirostris) off the Big Island of Hawai'i. Carmen Bazúa Durán (UNAM, Facultad de Ciencias, Depto. Física, Lab Acústica, Circuito exterior s/n, Cd. Universitaria, 04510 Mexico, D.F., Mexico, bazua@servidor.unam.mx), Nina V. Luna Valiente (UAM-Xochimilco, División de Ciencias Biológicas y de la Salud, 04960 Mexico, D.F., Mexico, bazua@servidor.unam.mx)

Many cetaceans live in coastal waters that are accessible to humans. In Kealakekua Bay, Hawai'i human activities occur during morning hours, when spinner dolphins rest and socialize (feeding occurs at night). Five human activities (swimmers, kayaks, motorized-boats, and narrow- or broadband engine noises) within $200 \mathrm{~m}$ of the dolphins were monitored to investigate their effect on dolphin behavior. Dolphin behavior was measured as aerial behavior (high, medium, low energy), acoustic behavior (whistles, burst-pulses, echoclick-trains), herd size, herd coordination (high, medium, low), and diving interval ( $\mathrm{DI}<1 \mathrm{~min}, 1 \mathrm{~min}<\mathrm{DI}<2 \mathrm{~min}, \mathrm{DI}>2 \mathrm{~min}$ ) in the presence or absence of each human activity. Interactions between human activities and dolphins occurred $71 \%$ of the time, having simultaneous presence of several human activities. When only one human activity was present, motorboats and broad-noise increased herd-coordination and number of aerial behaviors; motorboats and both engine-noises reduced number of acoustic behaviors; kayaks and narrow-noise increased herd-coordination, DI and some acoustic behaviors; swimmers reduced herd-coordination and increased number of acoustic behaviors. Therefore, all five human activities studied changed dolphin behavior and changed it differently. Spinner dolphins probably can distinguish efficiently between human activities and may be interrupting their rest cycle to warn each other, which could have effects on this species welfare.

\section{Invited Papers}

\section{$1: 40$}

1pAB3. Effects of sound on the behavior of toothed whales. Peter Tyack (Woods Hole Oceanographic Institution, Applied Ocean Physics \& Engineering Dept., Woods Hole, MA 02543, USA, ptyack@whoi.edu), Ian Boyd (Sea Mammal Research Unit, Gatty Marine Laboratory, University of St Andrews, KY16 8LB St Andrews, UK, ilb@st-andrews.ac.uk), Diane Claridge (Bahamas Marine Mammal Research Organisation, P.O. Box AB-20714, Abaco, 0 Marsh Harbour, Bahamas, bmms@oii.net), Christopher W. Clark (Cornell University Laboratory of Ornithology, Bioacoustics Research Program, 159 Sapsucker Woods Road, Ithaca, NY 14850, USA, cwc2@cornell.edu), David Moretti (NAVSEA, Newport Undersea Warfare Center, Newport, RI RI 02841, USA, MorettiDJ@npt.nuwc.navy.mil), Brandon Southall (National Marine Fisheries Service, 1315 East West Hwy, SSMC III \#12539, Silver Spring, MD 20910-6233, USA, Brandon.Southall@noaa.gov)

We report initial results from a study on behavioral responses of beaked and other whales to sonar and other sounds. This research is designed to provide new science-based approaches for mitigating the risk of sonar to beaked and other whales. The study was conducted at the Atlantic Undersea Test and Evaluation Center (AUTEC) range near Andros Island in the Bahamas, where Blainville's beaked whales (Mesoplodon densirostris) can regularly be detected using passive acoustic monitoring of their echolocation clicks. Tags recorded sound at the whale and behavior of the whale. Data were collected from 10 tag deployments, 6 on Blainville's beaked whales and 4 on pilot whales. 109 hours of data were collected from tags, 74 hours from beaked whales and 34 hours from pilot whales. 
Playbacks of mid-frequency sonar and killer whale calls were performed on 3 of the tagged whales, 1 beaked whale and 2 pilot whales. The tagged beaked whale responded to both sonar and killer whale sounds by premature cessation of clicking during foraging dives (RL $=\sim 117 \mathrm{~dB}$ re $1 \mu \mathrm{Pa}$ for the killer whale sound, $\sim 145 \mathrm{~dB}$ for the sonar), and an unusually slow and long ascent.

\section{2:00}

1pAB4. Acoustic flexibility in singing birds under noisy urban conditions. Hans Slabbekoorn (Leiden University, Kaiserstraat 63, 2311 GP Leiden, Netherlands, H.W.Slabbekoorn@Biology.LeidenUniv.NL)

Birds rely on song for defending a territory and attracting a mate. More and more breeding areas are affected by human activities and anthropogenic noise has become almost omnipresent. Consequently, song function in and around cities, and alongside highways, train tracks and airports, is increasingly hampered by noise interference. Several counter strategies in producing sounds, related to changes in loudness, pitch, and timing, can help out under these noisy urban conditions. Observational data have yielded several interesting patterns which show remarkable singing flexibility in different species that are typically common in cities. Here I present data for a new species, the chiffchaff (Phylloscopus collybita) showing spectral and temporal adaptation which reduces masking alongside a highway in the Netherlands. Furthermore, experimental field data reveal for the first time that this species is able to adjust immediately to exposure to highway noise via artificial playback in quiet territories. Acoustic flexibility may be key to efficient use of the 'left-over acoustic space' and may determine whether individual birds can maintain their territory and breed successfully. Variation in flexibility among species may explain which birds will remain widespread in our noisy world and which will be pushed back into 'silent' habitat pockets.

\section{Contributed Papers}

\section{2:20}

1pAB5. Comodulation masking release in the bottlenose dolphin (Tursiops truncatus). Brian K. Branstetter (US Navy Marine Mammal Program, Space and Naval Warfare Systems Center, 53560 Hull St., Code 71510, San Diego, CA 92152, USA, branstet@hawaii.edu), James J. Finneran (US Navy Marine Mammal Program, Space and Naval Warfare Systems Center, 53560 Hull St., Code 71510, San Diego, CA 92152, USA, james.finneran@navy.mil), Linda S. Green (Science Applications International Corporation, 4065 Hancock St., San Diego, CA 92110, USA, branstet@hawaii.edu), Erik E. Robinson (Science Applications International Corporation, 4065 Hancock St., San Diego, CA 92110, USA, branstet@hawaii.edu), Megan N. Tormey (Science Applications International Corporation, 4065 Hancock St., San Diego, CA 92110, USA, branstet@hawaii.edu), Randall L. Dear (Science Applications International Corporation, 4065 Hancock St., San Diego, CA 92110, USA, RANDALL.L .DEAR@saic.com)

The power spectrum model of masking, derived from critical band or critical ratio measurements is often cited and used to extrapolate the effects of masking noise on the signal detection abilities of marine mammals. However, the power spectrum model may not generalize well to environmental noise that is often coherently amplitude-modulated across frequency regions. The current study demonstrates a pattern of masking where temporally fluctuating comodulated noise produces lower masked thresholds (up to a $17 \mathrm{~dB}$ difference) compared to Gaussian noise of the same spectral density level. Noise possessing wide bandwidths, low temporal modulation rates, and across-frequency temporal envelope coherency resulted in lower masked thresholds, a phenomenon known as comodulation masking release. The results are consistent with a model where dolphins compare temporal envelope information across auditory filters to aid in signal detection. Furthermore, results suggest conventional models of masking derived from experiments using random Gaussian noise may not generalize well to environmental noise that dolphins actually encounter.

\section{2:40}

1pAB6. Investigating noise effects on the call amplitude of endangered Southern Resident killer whales (Orcinus orca). Marla Holt (NOAA Northwest Fisheries Science Center, Marine Mammal Program, 2725 Montlake Blvd East, Seattle, WA 98112, USA, Marla.Holt@noaa.gov), Val Veirs (Colorado College, Department of Physics, Colorado Springs, CO 80903, USA, vveirs@coloradocollege.edu), Scott Veirs (Beam Reach Marine Science and Sustainability School, 7044 17th Ave NE, Seattle, WA 98115, USA, scott@beamreach.org)

Anthropogenic sound exposure has been identified as a potential threat to endangered Southern Resident killer whales (SRKWs). Such exposure can mask important biological sounds including those used for social functions. Vocal animals might compensate for increased background noise by calling louder (the Lombard effect). In this study, amplitude compensation was investigated in SRKW communication calls recorded off of San Juan Island, WA, U.S.A. Data were obtained from two calibrated recording systems, a shore-based hydrophone array [V. Veirs, J. Acoust. Soc. Am., 116, 2615 (2004)] and a boat-based vertical array. Calls were localized using time of arrival differences to estimate range and apparent source levels after background levels were subtracted from the signal. Call levels were calculated assuming spreading loss functions determined from sound propagation observations made in situ. Results demonstrated a positive relationship between call levels and background levels. Costs of such vocal flexibility are unknown, but may include changes in energetic demand and/or communicative functionality. Within the data ranges measured in this study, killer whales communicatively adjusted for fluctuating background noise levels. However, other sounds detected during passive listening (e.g. prey sounds) might be more susceptible to masking effects [Work supported by NOAA and Colorado College.]

\section{Invited Papers}

1pAB7. Effects of noise on hearing in odontocetes. James J. Finneran (US Navy Marine Mammal Program, Space and Naval Warfare Systems Center, 53560 Hull St., Code 71510, San Diego, CA 92152, USA, james.finneran@navy.mil), Carolyn E. Schlundt (EDO Professional Services, 3276 Rosecrans St., San Diego, CA 92110, USA, carolyn.melka@edocorp.com), Sam H. Ridgway (University of California, San Diego, Dept. of Pathology, School of Medicine, 9500 Gilman Drive, La Jolla, CA 92093, USA, ridgway @ spawar.navy.mil)

Increased public concern and regulation of activities involving anthropogenic sound have resulted in a pressing need for specific information regarding safe limits for marine mammals exposed to underwater noise. Since many marine mammals have sensitive hearing and rely upon underwater sound for communicating, foraging, and navigating, the potential effects of noise on their hearing is of particular concern. One of the most familiar consequences of noise exposure is an increase in threshold that persists after the cessation of the noise, called a temporary thresholds shift (TTS) or permanent threshold shift (PTS). Although there are no PTS data for marine 
mammals, there have been TTS measurements in a number of species, including bottlenose dolphins and belugas. These studies compare hearing thresholds before and after subjects are exposed to intense sounds. The results are analogous to data from terrestrial mammals, where TTS depends on the exposure frequency, sound pressure, duration, and temporal pattern. This talk reviews the major findings related to the growth of and recovery from TTS in bottlenose dolphins and belugas and discusses the application of these data to acoustic exposure guidelines.

$3: 20$

1pAB8. Noise-induced permanent threshold shift in a harbor seal. David Kastak (UCSC Institute of Marine Sciences, Long Marine Lab - University of California, 100 Shaffer Road, Santa Cruz, CA 95060, USA, kastak@ucsc.edu), Jason Mulsow (UCSC Institute of Marine Sciences, Long Marine Lab - University of California, 100 Shaffer Road, Santa Cruz, CA 95060, USA, jmulsow@ucsc.edu), Asila Ghoul (UCSC Institute of Marine Sciences, Long Marine Lab - University of California, 100 Shaffer Road, Santa Cruz, CA 95060, USA, asila@ucsc.edu), Colleen Reichmuth (UCSC Institute of Marine Sciences, Long Marine Lab - University of California, 100 Shaffer Road, Santa Cruz, CA 95060, USA, coll@ucsc.edu)

Investigation of auditory temporary threshold shift (TTS) in marine mammals has provided a means of predicting the harmful effects of underwater anthropogenic noise. As complete recovery of hearing is requisite in these studies, they have been considered appropriate in light of subject availability and ethical considerations. In our psychophysical studies of TTS in pinnipeds, we have employed methods designed to safely titrate from sound levels of noise inducing no TTS to levels of significant but completely recoverable hearing loss. In the present study, these methods were used with a harbor seal (Phoca vitulina) exposed to an underwater $4.1 \mathrm{kHz}$ pure tone fatiguing stimulus. Sound levels and durations were gradually increased to a maximum received sound pressure of $184 \mathrm{~dB}$ re $1 \mu \mathrm{Pa}$ with a duration of $60 \mathrm{~s}\left(\mathrm{SEL}=202 \mathrm{~dB}\right.$ re $\left.1 \mu \mathrm{Pa}^{2} \mathrm{~s}\right)$. Upon the second exposure to this fatiguing stimulus, an initial threshold shift in excess of $50 \mathrm{~dB}$ was estimated at a test frequency of $5.8 \mathrm{kHz}$, a half-octave above the fatiguing tone. Recovery from this unexpectedly large shift occurred at a rate of $-10 \mathrm{~dB}$ per $\log (\mathrm{min})$, with an apparently permanent threshold shift of 7 to $10 \mathrm{~dB}$ evident after more than two months following exposure.

\section{3:40-4:00 Break}

\section{4:00}

1pAB9. Anthropogenic sounds - Potential effects on fish. Arthur Popper (Dept. of Biology, University of Maryland, College Park, MD 20742, USA, apopper@umd.edu), Svein Løkkeborg (Fish Capture Division, Institute of Marine Research, P.O. Box 1870, Nordnes N-5817 Bergen, Norway, svein.loekkeborg@imr.no), Robert McCauley (Curtin University, Centre for Marine Science and Technology, GPO Box U 1987, 6845 Perth, Australia, r.mccauley@cmst.curtin.edu.au)

There is concern that human-generated sounds may have deleterious effects on fish. This paper will review some of what is currently known about these effects, and consider the questions that have to be answered before developing models to enable "prediction" of sound effects on particular fish species. A major restriction is that there are few peer-reviewed data on effects of anthropogenic sources on fish. Extrapolation from these results is further confounded since experiments differ in many ways, each of which may alter the resultant impact on fish. For example, studies vary in sounds types tested (e.g., pile driving vs. ship noise), signal parameters (intensity, number of repetitions), species used, fish age, etc. Moreover, a singularly important issue is that while many of the issues and impact mechanisms are potentially amenable to experimental lab study, the ultimate questions regarding the effects of sound on fish behavior need to field based and require long-term observations where behaviour of wild fish is not constrained. Only by observing fish in the wild will we ultimately understand if, and how, anthropogenic sounds impact fish both during exposure and, more importantly, for extended periods after the termination of the sound.

\section{4:20}

1pAB10. Characterizing the relative contributions of large vessels to total ocean noise fields: a case study using the Gerry E. Studds Stellwagen Bank National Marine Sanctuary. Leila T. Hatch (US NOAA, Stellwagen Bank National Marine Sanctuary, 175 Edward Foster Road, Scituate, MA 02066, USA, leila.hatch@noaa.gov), Christopher W. Clark (Cornell University Laboratory of Ornithology, Bioacoustics Research Program, 159 Sapsucker Woods Road, Ithaca, NY 14850, USA, cwc2@cornell.edu), Sofie Van Parijs (US NOAA, Northeast Fisheries Science Center, 166 Water Street, Woods Hole, MA 02543, USA, sofie.vanparijs@noaa.gov), Richard Merrick (US NOAA, Northeast Fisheries Science Center, 166 Water Street, Woods Hole, MA 02543, USA, richard.merrick@noaa.gov), Dimitri Ponirakis (Cornell University Laboratory of Ornithology, Bioacoustics Research Program, 159 Sapsucker Woods Road, Ithaca, NY 14850, USA, dwp22@cornell.edu), Kurt Schwehr (University of New Hampshire, Center for Coastal and Ocean Mapping Joint Hydrographic Center, 24 Colovos Road, Durham, NH 03824, USA, kurt@ccom.unh.edu), Michael A. Thompson (US NOAA, Stellwagen Bank National Marine Sanctuary, 175 Edward Foster Road, Scituate, MA 02066, USA, michael.a.thompson@noaa.gov), David Wiley (US NOAA, Stellwagen Bank National Marine Sanctuary, 175 Edward Foster Road, Scituate, MA 02066, USA, david.wiley@noaa.gov)

Understanding and mitigating the effects of underwater noise on marine species requires substantial information regarding acoustic contributions from shipping. In 2006, we used the U.S. Coast Guard's Automatic Identification System (AIS) to describe patterns of large commercial ship traffic within a U.S. National Marine Sanctuary. AIS data were combined with low-frequency acoustic data from an array of nine-ten autonomous recording units deployed throughout 2006. Analysis of received sound levels (10-1000 Hz, root-mean squared decibels re $1 \mu$ Pascal \pm standard error) averaged $119.5 \pm 0.3$ at high traffic locations. High traffic locations experienced double the acoustic power of less trafficked locations for the majority of the time period analyzed. Average source level estimates $(71-141 \mathrm{~Hz}$, root-mean squared decibels re $1 \mu$ Pascal \pm standard error) for individual vessels ranged from $158 \pm 2$ (research vessel) to $186 \pm 2$ (oil tanker). Tankers were estimated to contribute two times more acoustic power to the region annually than cargo ships, and over one 
hundred times more than research vessels. Our results indicate that noise produced by large commercial traffic was at levels and within frequencies that warrant concern among managers regarding the ability of endangered whales to maintain acoustic contact within greater sanctuary waters.

\section{Contributed Papers}

\section{4:40}

1pAB11. Estimates of chronic SNR exposure levels of sperm whales (Physeter macrocephalus) to airguns in the Gulf of Mexico. Aaron Thode (Marine Physical Laboratory, Scripps Institution of Oceanography, 9500 Gilman Dr, MC 0238, La Jolla, CA 92093-0238, USA, athode@ucsd.edu), Martha Winsor (Hatfield Marine Science Center, Oregon State University, Newport, CA 97365, USA, martha.winsor@oregonstate.edu), Bruce Mate (Hatfield Marine Science Center, Oregon State University, Newport, CA 97365, USA, bruce.mate@oregonstate.edu), Matt Howard (Dept. of Oceanography, Texas A\&M University, 3146 TAMU, College Station, TX 77843, USA, mkhoward@tamu.edu), Katherine Kim (HLS Research, Inc., 3366 N. Torrey Pines Ct., Ste. 310, La Jolla, CA 92037, USA, Katherine.Kim@HLSResearch.com), John_Diebold (Lamont-Doherty Earth Observatory of Columbia University, 61 Route 9W, Palisades, NY 10964-8000, USA, Johnd@ldeo.columbia.edu), Maya Tolstoy (Lamont-Doherty Earth Observatory of Columbia University, 61 Route 9W, Palisades, NY 10964-8000, USA, tolstoy@1deo.columbia.edu)

A synthesis effort by the Sperm Whale Seismic Study (SWSS) has estimated chronic levels of low-level, long-term acoustic exposures of sperm whales in the Gulf of Mexico due to seismic airgun activity. Between 2002 and 2005, satellite-tags were attached to 53 whales of both sexes in the northern Gulf, with an average tag viability of 187 days. Data provided by the International Association of Geophysical Contractors (IAGC) permitted the range estimation of active seismic vessels to each whale location. The ray-tracing model BELLHOP was used to estimate received levels from each seismic vessel for every space-time data point at frequencies between 10 to $1000 \mathrm{~Hz}$, using simulated source signatures from a 3590 in 3 seismic array. Winds speeds from numeric models at each space-time location were converted into ambient noise levels, and shipping noise levels were crudely estimated. The preliminary synthesis using a simple spherical spreading transmission loss model suggests that over $95 \%$ of the whale positions reported during times of airgun activity occurred in circumstances where the received airgun pulses had $0 \mathrm{~dB}$ signal-to-noise ratio (SNR) or greater compared to ambient levels, and between $25-60 \%$ of all locations have received SNRs of $20 \mathrm{~dB}$ or greater, depending on the SNR definition used. [Sponsored by US Minerals Management Service].

\section{5:00}

1pAB12. Underwater radiated noise due to the piling for the Q7 Offshore Wind Park. Christ A.f. De Jong (TNO Science and Industry, P.O. Box 155, 2600 AD Delft, Netherlands, christ.dejong@tno.nl), Michael A. Ainslie (TNO Defence, Security and Safety, Oude Waalsdorperweg 63, 2597 AK The Hague, Netherlands, michael.ainslie@tno.nl)

The Q7 is the second offshore wind farm in the Dutch sector of the North Sea and, at $23 \mathrm{~km}$ off the Dutch coast, the world's first to be located outside the 12-mile limit. To support the wind turbines, monopiles (54 metres long steel pipes with a diameter of 4 metres) are hammered into the seabed using a pile-driver. The underwater radiated noise during the impulsive hammering of 9 out of 61 monopiles was monitored. Although there is a wide concern about the impact of piling noise on the marine life in the area, there are no criteria for the maximum acceptable noise levels. A quantitative comparison of the results of various studies in which the underwater noise from pile driving has been measured and reported is difficult, due to the lack of standardization in the level definitions and data processing. The Q7 data have been analyzed in terms of a broadband sound exposure level, peak pressure and pulse duration and a 1/3-octave band frequency spectrum of the sound pressure at different hydrophone locations, for each hammer stroke that has been recorded. The results are discussed in relation to the stroke energy and the hydrophone distance and depth.

\section{5:20}

1pAB13. Monitoring the temporal and spatial characteristics of the noise radiated from marine piling. Paul A. Lepper (Loughborough University, Electronic \& Electrical Engineering, LE11 3TU Leicestershire, UK, p.a.lepper@lboro.ac.uk), Stephen P. Robinson (National Physical Laboratory, Hampton Road, TW11 OLW Teddington, UK, Stephen.Robinson@npl .co.uk)

Noise is often an unintended by-product of offshore activities, and a significant source of impulsive underwater noise is marine piling, where a pile is driven into the sea-bed using a hydraulic hammer. This paper describes work undertaken to monitor the underwater radiated noise during offshore marine piling. The measurements made include full characterisation of the temporal variation of the resulting sound field, including frequency content of the pulses and the increasing amplitude typically observed during the soft start period. Results are also shown for the spatial variation of the field, including the effect on propagation of significant bathymetry changes (for example, due to the presence of a sand bank). The energy within each acoustic pulse is an appropriate measure of the sound field, having the advantage that it is relatively straightforward to add the contributions from each pulse in order to derive an overall sound exposure level (SEL) in a manner analogous to methods familiar from air acoustics. An example is then given of how the measured data and predictive modeling may be used to calculate an overall exposure for an animal in the vicinity, using various assumptions about the location and mobility of the animal.

\section{$5: 40$}

1pAB14. Monitoring, measuring and describing ocean noise over ecologically viable scales with applications to impacts on large whales. Christopher W. Clark (Cornell University Laboratory of Ornithology, Bioacoustics Research Program, 159 Sapsucker Woods Road, Ithaca, NY 14850, USA, cwc2@cornell.edu), Chris Tremblay (Cornell University Laboratory of Ornithology, Bioacoustics Research Program, 159 Sapsucker Woods Road, Ithaca, NY 14850, USA, cjt35@cornell.edu), Melissa Fowler (Cornell University Laboratory of Ornithology, Bioacoustics Research Program, 159 Sapsucker Woods Road, Ithaca, NY 14850, USA, mcf3@cornell.edu), Christi Diamond (Cornell University Laboratory of Ornithology, Bioacoustics Research Program, 159 Sapsucker Woods Road, Ithaca, NY 14850, USA, cd254@cornell.edu), Dimitri Ponirakis (Cornell University Laboratory of Ornithology, Bioacoustics Research Program, 159 Sapsucker Woods Road, Ithaca, NY 14850, USA, dwp22@cornell.edu), Clara McCarthy (Cornell University Laboratory of Ornithology, Bioacoustics Research Program, 159 Sapsucker Woods Road, Ithaca, NY 14850, USA, cmm255@cornell.edu), Janelle Morano (Cornell University Laboratory of Ornithology, Bioacoustics Research Program, 159 Sapsucker Woods Road, Ithaca, NY 14850, USA, jlm394@cornell.edu), Charles Muirhead (Cornell University Laboratory of Ornithology, Bioacoustics Research Program, 159 Sapsucker Woods Road, Ithaca, NY 14850, USA, cam236@cornell.edu), Anita Murray (Cornell University Laboratory of Ornithology, Bioacoustics Research Program, 159 Sapsucker Woods Road, Ithaca, NY 14850, USA, am678@cornell.edu), Liz Rowland (Cornell University Laboratory of Ornithology, Bioacoustics Research Program, 159 Sapsucker Woods Road, Ithaca, NY 14850, USA, edr6@cornell.edu), Catherine Vannicola (Cornell University Laboratory of Ornithology, Bioacoustics Research Program, 159 Sapsucker Woods Road, Ithaca, NY 14850, USA, cmv44@cornell.edu), Ann Warde (Cornell University Laboratory of Ornithology, Bioacoustics Research Program, 159 Sapsucker Woods Road, Ithaca, NY 14850, USA, amw38@cornell.edu)

Large whales communicate primarily in the low $(<1000 \mathrm{~Hz})$ frequency bands; the same frequency range within which anthropogenic ocean noise has been increasing over the last half century at approximately 3-5 $\mathrm{dB} /$ decade. The working assumption and hypothesis hold that rising ambient noise levels negatively impact whales by interfering with communication, navigation and predator detection, and that over the long-term such effects 
could reduce breeding success and population size. There is a lack of empirical data on the spatial-temporal dynamics of ambient noise by which to assess potential impacts. One year of continuous acoustic data from an ongoing distributed array of 19 continuous seafloor recorders covering a 1000 $\mathrm{km}^{2}$ area in Massachusetts Bay, USA were analyzed for ambient noise statistics and the occurrences, locations and vocal behaviors of fin, humpback and right whales. Noise levels were greater than $120 \mathrm{~dB}$ rms re $1 \mu \mathrm{Pa}$ in the $10-1000 \mathrm{~Hz}$ band throughout the $1000 \mathrm{~km}^{2}$ area for $\geqslant 50 \%$ of the season when whales were present. If animals are relying on acoustic cues to coordinate feeding and social interactions, these high noise levels impose a significant loss of acoustic habitat during the whales' residency in the area.

\section{6:00}

1pAB15. Environmental Risk Management Capability: Advice on minimising the impact of sonar on marine mammals. Colin Schofield (BAE Systems Integrated System Technologies, Unit D1, Marabout Industrial Estate, DT1 1YA Dorchester, UK, colin.schofield @baesystems.com)

The Environmental Risk Management Capability - Sonar (ERMC(S)) has now been accepted into service by the UK Royal Navy. ERMC(S) is the first operational software package that provides a comprehensive risk assessment of the common concern of the potential impact of sonar usage on marine mammals. The algorithms include allowance for extended periods of use and advice on achievable mitigation options. Now that the system has achieved this significant milestone, this paper reviews the elements of the system that have made progress to this stage so successful: -The techniques and data used by the algorithms. -The inclusive development approach that allowed all the concerned stakeholders to be satisfied with the processes utilised. -The annual review cycle that will enable the tool to maintain its links with forefront research in this area. -The application of the system to non-military sound sources as the benchmark approach. This paper will show how ERMC(S) can be develop into a tool for both commercial and military sound sources using proving and established techniques.

\section{$6: 20$}

1pAB16. Proposed Marine Mammal Noise Exposure Criteria: Current Data Base, Limitations, and Research Needs. Darlene R. Ketten (Woods Hole Oceanographic Institution, Applied Ocean Physics \& Engineering Dept., Woods Hole, MA 02543, USA, dketten@whoi.edu), Ann E. Bowles (Hubbs-Sea World Research Institute, 2595 Ingraham Street, San Diego, CA 92109, USA, abowles@ @swri.com), William T. Ellison (Marine Acoustics, Inc, 809 Aquidneck Ave, Middletown, RI 02842, USA, WEMAI@aol.com), James J. Finneran (US Navy Marine Mammal Program, Space and Naval Warfare Systems Center, 53560 Hull St., Code 71510, San Diego, CA 92152, USA, james.finneran@navy.mil), Roger L. Gentry (ProScience Consulting, LLC, P.O. Box 177, Dickerson, MD 20842-0177, USA, roger.gentry@comcast.net), Charles R. Greene (Greeneridge Sciences, Inc., 4512 Via Huerto, Santa Barbara, CA 93110, USA, cgreene@greeneridge.com), David Kastak (UCSC Institute of Marine Sciences, Long Marine Lab - University of California, 100 Shaffer Road, Santa Cruz, CA 95060, USA, kastak@ucsc.edu), James H.
Miller(University of Rhode Island, Department of Ocean Engineering, Narragansett Bay Campus, Narragansett, RI 02882, USA, miller@egr.uri.edu), Paul E. Nachtigall (University of Hawaii, Hawaii Institute of Marine Biology, P.O. Box 1106, Kailua, HI 96734, USA, nachtiga@hawaii.edu), W J. Richardson (LGL Ltd., Environmental Research Associates, P.O. Box 280, 22 Fisher St, King City, AB L7B 1A6, Canada,wjr@1gl.com), Brandon Southall (National Marine Fisheries Service, 1315 East West Hwy, SSMC III \#12539, Silver Spring, MD 209106233, USA, Brandon.Southall@noaa.gov), Jeanette A. Thomas (Western Illinois University Quad Cities, Department of Biological Sciences, 3561 60th St., Moline, IL 61265, USA, j-thomas@wiu.edu), Peter Tyack (Woods Hole Oceanographic Institution, Applied Ocean Physics \& Engineering Dept., Woods Hole, MA 02543, USA, ptyack@whoi.edu)

This paper presents the findings of an inter-disciplinary expert panel based on comprehensive review of hearing and noise effects data for marine and land mammals. The principal motivation for the review was to assist formulation of data-based noise exposure criteria in light of rapidly evolving research advances in marine mammal hearing and underwater noise effects. Levels estimated to induce permanent hearing loss were determined for single exposure events for cetaceans (in water) and pinnipeds (in air and water) for each of 15 sound type/animal group combinations. These recommendations represent a current best estimate only and are modular, with modifiable key variables; e.g., source and exposure, to facilitate revision as data improve. In some cases, relatively explicit injury limits are proposed, e.g., $186 \mathrm{~dB}$ re: $1 \mu \mathrm{Pa}^{2}$-s (frequency-weighted sound exposure level) and 218 $\mathrm{dB}$ re: $1 \mu \mathrm{Pa}_{\text {peak }}$ (unweighted peak sound pressure level) for pinnipeds in water exposed to multiple sound pulses. In others, particularly for behavioural effects of multiple-pulse and non-pulse exposures, response severity and significance are quantitatively scored, but the data do not allow identification of specific broadly-applicable disturbance thresholds. These findings are a current best effort and include a discussion of limitations and recommended research needed to address data gaps.

\section{$6: 40$}

1pAB17. A simple acoustical exposure metric based on biological thresholds and integrating a temporal characteristics exposure axis. Michael Stocker (Ocean Conservation Research, P.O. Box 559, Lagunitas, CA 94938, USA, mstocker@OCR.org), Tom Reuterdahl (505 LaVerne Ave, Mill Valley, CA 94941, USA, euler1@sbcglobal.net), Libbie Horn (975 Black Mountain Rd., Hillsborough, CA 94-1-, USA, libbie.horn@gmail.com), Gail Hurley (768 Lovell Ave., Mill Valley, CA 94941, USA, hurleyitaly@hotmail.com)

Anthropogenic noise is compromising habitat for marine mammals, fish and potentially other marine organisms. Determining acceptable exposure thresholds is confounded by the fact that marine animals have adapted to some exceedingly loud naturally occurring sounds, while exposure to certain anthropogenic noises at equivalent or lower amplitudes causes harm. It is clear that mitigation levels can not be established by signal amplitude alone and that other signal characteristics are significant factors in biological responses to noise exposure. This proposed metric continues ongoing work on a simple exposure metric based on broadband frequency and amplitude representation of a subject noise with the time domain represented in the $\mathrm{Z}$ axis. 


\title{
Session 1pAOa
}

\section{Acoustical Oceanography and ECUA: Acoustical Oceanography of Polar Environments I}

\author{
Peter N. Mikhalevsky, Cochair \\ Science Applications International Corporation, 4001 N. Fairfax Dr. Suite 850, Arlington, VA 22203, USA \\ Hanne Sagen, Cochair \\ Nansen Environmental and Remote Sensing Center, Thormolensgt. 47, Bergen, 5006, Norway
}

\section{Invited Papers}

\author{
1:00
}

1pAOa1. Acoustic communication and navigation in the Arctic Ocean. Arthur B. Baggeroer (MIT, Department of Mathematics, 77 Massachusetts Avenue, Cambridge, MA 02139, USA, abb@boreas.mit.edu), Lee Freitag (Woods Hole Oceanographic Inst., 266 Woods Hole Road, MS 18, Woods Hole, MA 02543, USA, Ifreitag@ whoi.edu), James E. Priesig (Woods Hole Oceanographic Inst., 266 Woods Hole Road, MS 18, Woods Hole, MA 02543, USA, jreisig@whoi.edu), Daniel Nagle (Naval Undersea Systems Center, 1176 Howell Street, Newport, RI 02841, USA, nagledt@npt.nuwc.navy.mil)

Acoustic communication and navigation in the Arctic usually implies surface interaction because of the upward refracting profile. While open water leeds and polyna exist, sensors must also be deployed beneath the ice. Nominal roughness of several meters plus ubiquitous ice keels all lead to complicated multipath propagation typically of durations from 0.1 to 10 seconds. Pack ice drifts at scales of $0.1-0.2 \mathrm{~nm} / \mathrm{s}$ leading to doppler shifts/spreads of $2-8 \mathrm{~Hz}$ at $10-20 \mathrm{kHz}$ carriers. These lead to highly spread channels with lots of ISI. Wise signal and code designs coupled with equalizers are critical to successful performance of acomms system even at low rates. Navigation such as by long base line systems encounter the same multipath. Path detectors and trackers are easily confused in determining interactions with the underside of the ice cover. Often, first arrivals are weak and do not trigger detectors. This leads to "lane" skipping by tracking algorthms and sychonization errors for acomms. This implies clever "track association" algorithms to identify the arrival patterns and then transform them to locations which move with ice drift. This presentation outlines acoustic problems which communicaton and navigation systems must overcome using data take from several Arctic experiments.

$1: 20$

1pAOa2. Propagation, scattering and reverberation in an ice-covered Arctic ocean. Henrik Schmidt (MIT, 77 Mass Ave, 5-204, Cambridge, MA 02139, USA, henrik@mit.edu), Kevin D. Lepage (Naval Research Laboratory, 4555 Overlook Ave SW, Washington, DC 20375, USA, kevin.lepage@nrl.navy.mil)

The Arctic Ocean is a unique acoustic environment due to the ice cover and the strongly upward refracting sound speed profile. A large amount of theoretical and experimental research has focused on the significance of the scattering of sound by the rough ice cover. In spite of this, a strong anomali persisted until the early 1990s in the propagation of low-frequency sound in particular, with prevailing rough-surface scattering theories incapable of explaining the frequency dependence of transmission loss observed experimentally. More recent modeling was cabable of reproducing the observed transmission losses by combining the effects of ice elasticity, rough interface scattering, and waveguide propagation [JASA, 96:1783-1795,1994]. Thus, it was demonstrated that incoherent scattering into Lamb waves in the ice provided a significant loss mechanism. This mechanism itself did not explain the frequency dependence, but when incorporating the bilinear sound speed profile of the arctic ocean, it was found that the lower-order modes become disproportionately attenuated, explaining the high attenuation observed at low frequencies. The finding was later confirmed by succesfully modeling the modal losses observed in 1994 Trans-Arctic Propagation experiments, and the ability of the same model to accurately predict observed, long-range reverberation measurements [JASA, 111:747-760,2001]. [Work supported by ONR.]

\section{$1: 40$}

1pAOa3. Long-term variations of ice breaking noise in Antarctica. Alexander Gavrilov (Curtin University of Technology, Centre for Marine Sci \& Tech, GPO Box U1987, 6845 Perth, WA, Australia, a.gavrilov@cmst.curtin.edu.au), Binghui Li (Curtin University of Technology, Centre for Marine Sci \& Tech, GPO Box U1987, 6845 Perth, WA, Australia, binghui.li@postgrad.curtin.edu.au)

Numerous low frequency transient signals arriving from Antarctica have been detected in five-year sea noise recordings in 20022006 at the hydroacoustic listening station deployed off Cape Leeuwin in Western Australia as part of the International Monitoring System of the Comprehensive Nuclear-Test-Ban Treaty. The majority of these signals consist of a single arrival of mode 1 which dominates the higher modes and undergoes strong frequency dispersion due to long-range propagation in the polar ocean environment. These signals are believed to be emitted primarily by ice rifting and breaking events on the Antarctic shelf. Several regions along the Antarctic coast associated with certain glaciers and ice tongues were found to persistently be much more active in generating ice noise than other 
parts of the Antarctic shelf. An analysis of long-term changes in the frequency of occurrence of ice events showed noticeable seasonal cycles, but did not reveal any significant interannual variations against the background of short-term fluctuations. Some of the ice events detected at the Cape Leeuwin station were also identified in sea noise recordings made on autonomous acoustic loggers deployed on the Antarctic shelf, which allowed us to investigate characteristics of the acoustic signals from ice events near their origins.

\section{Contributed Papers}

\section{2:00}

1pAOa4. Localization of Antarctic ice breaking events by frequency dispersion of the signals received at a single hydroacoustic station in the Indian Ocean. Binghui Li (Curtin University of Technology, Centre for Marine Sci \& Tech, GPO Box U1987, 6845 Perth, WA, Australia, binghui.li@postgrad.curtin.edu.au), Alexander Gavrilov (Curtin University of Technology, Centre for Marine Sci \& Tech, GPO Box U1987, 6845 Perth, WA, Australia, a.gavrilov@cmst.curtin.edu.au)

Transient acoustic signals from Antarctic ice cracking and breaking events, featuring significant frequency dispersion, were observed at the hydroacoustic stations deployed in the Indian Ocean as part of the International Monitoring System (IMS) of the Comprehensive Nuclear-Test-Ban Treaty. Based on a comparison with numerical predictions, the measured dispersion characteristics were used to estimate the range between ice events and the receiver. Combined with the bearing capability of the IMS stations, these estimates allow us to locate ice events from a single hydroacoustic station. An analysis of range estimation errors due to uncertainty of the measured time-frequency structure of signal arrivals and due to variations of the sound speed profiles was also conducted. The analysis showed that the location accuracy from a single hydroacoustic station for the typical ice events was of the same order, as that determined from an intersection of bearings from two remote stations, if the signal frequency bandwidth is as wide as at least $5 \mathrm{~Hz}$ and lies within $5 \mathrm{~Hz}$ to $35 \mathrm{~Hz}$ frequency range. This localization method was examined by analysing several ice events detected at both the Cape Leeuwin IMS station and a sea noise logger deployed off the Antarctic shelf.
$2: 20$

1pAOa5. Year-round acoustic monitoring of large whales in polar environments: a comparison of Davis and Bransfield Straits. Kathleen Stafford (University of Washington Applied Physics Lab, 1013 40th St NE, Seattle, WA 98105, USA, stafford@apl.washington.edu), Malene J. Simon (University of Aarhus, Institute of Biology, C.F. Mollers Alle 1131, 8000 Aarhus C, Denmark, MaSi@natur.gl), Robert Dziak (Oregon State Univ. and NOAA, 2030 SE Marine Science Dr., Newport, OR 97365, USA, robert.p.dziak@noaa.gov), David K. Mellinger (Oregon State Univ. and NOAA, 2030 SE Marine Science Dr., Newport, OR 97365, USA, David .Mellinger@oregonstate.edu)

Both Davis Strait, in the Arctic between Canada and Greenland, and Bransfield Strait, in the Antarctic between the Antarctic Peninsula and the South Shetland Islands, are areas that experience extreme inter-seasonal differences in temperature, ice cover and productivity. The seasonally productive nature of both of these ice-influenced areas provides habitat for foraging large whales such as blue (Balaenoptera musculus) and fin (B. physalus) whales. In order to monitor and compare the seasonal occurrence of these species at high latitude regions at opposite ends of the globe, autonomous underwater hydrophone packages were deployed for one year in each Strait. Three instruments were deployed and recovered in Davis Strait from October 2006-07 and five in Bransfield Strait from November 2006-07. Blue and fin whales were recorded seasonally in both regions and exhibited the geographic acoustic distinctions between northern and southern hemisphere populations. Comparisons of the seasonal occurrence and the influence of ice cover on this occurrence will be compared and contrasted between north and south.

\section{Invited Papers}

1pAOa6. Acoustic navigation and communications for high latitude ocean research (ANCHOR). Craig M. Lee (Applied Physics Laboratory, University of Washington, 1013 NE 40th St, Seattle, WA 98105-6698, USA, craig @apl.washington.edu), Jason I. Gobat (Applied Physics Laboratory, University of Washington, 1013 NE 40th St, Seattle, WA 98105-6698, USA, jgobat@apl .washington.edu)

Recent community reports on autonomous and Lagrangian platforms and Arctic observing identify the development of under-ice navigation and telemetry technologies as one of the critical factors limiting the scope of autonomous (e.g. floats, AUVs and gliders) high-latitude measurement efforts. These platforms could provide persistent, high-resolution, basin-wide sampling in ice-covered regions and collect measurements near the critical ice-water interface. Motivated by the dramatic advances in temporal and spatial reach promised by autonomous sampling and by the need to coordinate nascent efforts to develop navigation and communication system components, an international group of acousticians, platform developers, high-latitude oceanographers and marine mammal researchers gathered in Seattle, U.S.A. from 27 February - 1 March for an NSF Office of Polar Programs sponsored Acoustic Navigation and Communication for High-latitude Ocean Research workshop. Workshop participants summarized the current state of knowledge concerning Arctic acoustics, navigation and communications, developed an overarching system specification to guide community-wide engineering efforts and established an active community and steering group to guide long-term efforts and ensure interoperability between elements developed by disparate teams. This presentation will summarize workshop findings and provide an update on recent developments stemming from the EU DAMOCLES and US NSF Arctic Observing Network programs.

\section{3:00}

1pAOa7. Sound fixing and ranging beneath sea-ice. Jean-Claude Gascard (University Pierre \& Marie Curie, 4 place Jussieu, Locean tour 45-46, 5e, 75005 Paris, France, jga@locean-ipsl.upmc.fr)

Sound fixing and ranging techniques, the so-called SOFAR technique, is widely used in the field of oceanography for tracking neutrally buoyant floats underwater over large distances (several thousands of kilometres) and for long periods of time (several years). The range propagation depends largely on the existence or not of the SOFAR sound channel. Twenty years ago we investigated SOFAR acoustic propagation under sea-ice over large distances and at various frequencies from $80 \mathrm{~Hz}$ up to $1560 \mathrm{~Hz}$. We discovered the range for acoustic propagation was much reduced (100 kms to $150 \mathrm{kms}$ ) no matter the frequency used. This was due to large scattering induced by under sea-ice topography since there is no way to avoid acoustic rays to bounce back to the surface towards sea-ice and create multiple reflections. More recently during the Tara Damocles transpolar drift across the Arctic Ocean, we reinvestigated the 
SOFAR acoustic range propagation under sea-ice for longer periods of time in various conditions (summer and winter conditions). Our results confirmed those obtained 20 years ago and indicated a high sensitivity to the ambient conditions. We are presenting the results obtained during the late fall- early winter months of the Tara transpolar drift across the Arctic Ocean.

\title{
Contributed Papers
}

3:20

1pAOa8. The Fram Strait acoustic tomography system. Hanne Sagen (Nansen Environmental and Remote Sensing Center, Thormølensgt. 47, 5006 Bergen, Norway, hanne.sagen@nersc.no), Stein Sandven (Nansen Environmental and Remote Sensing Center, Thormølensgt. 47, 5006 Bergen, Norway, stein.sandven@nersc.no), Peter Worcester (MPL, Scripps Institution of Oceanography, University of California, San Diego, La Jolla, CA 92093-0238, USA, pworcester@ucsd.edu), Matthew Dzieciuch (MPL, Scripps Institution of Oceanography, University of California, San Diego, La Jolla, CA 92093-0238, USA, mad@ucsd.edu), Emmanuel Skarsoulis (FORTH/IACM, N. Plastira 100, Vasilika Voutes, GR-70013 Heraklion, Greece, eskars@iacm.forth.gr)

The deep and wide Fram Strait between Greenland and Spitzbergen is the main influx and efflux gate to the Arctic Basin. Although major resources are invested in measurements of current and temperature here (http: //asof.npoar.no), the flux estimates still have significant deficiencies and errors. Our objective is to build, test, validate and use an innovated integrated observing and modeling system, including acoustic tomography, for improved monitoring of volume, heat and freshwater transports in the Fram Strait. As part of the DAMOCLES-IP, (=Developing Arctic Modeling and Observing Capabilities for Long-term Environmental Studies - Integrated Project) a first step acoustic tomography system is to be installed in the Fram Strait between East Greenland and West Spitzbergen in August 2008. The first step tomography system consists of one acoustic source near the Svalbard shelf and one receiver array in the middle of the Fram Strait. An extended acoustic system serving both tomography and glider navigation is planned implemented in 2009 within the recently funded project "Acoustic technology for observing the interior of the Arctic Ocean" (ACOBAR). We will present the specification of the tomography systems; our experimental plans, and plans for data analysis, including data assimilation.

\section{$3: 40$}

1pAOa9. Ocean acoustic tomography: Travel-time inversion in the eastern Fram Strait. Emmanuel Skarsoulis (FORTH/IACM, N. Plastira 100, Vasilika Voutes, GR-70013 Heraklion, Greece, eskars@iacm.forth.gr), George Piperakis (FORTH/IACM, N. Plastira 100, Vasilika Voutes, GR-70013 Heraklion, Greece, piperak@iacm.forth.gr), Michael Kalogerakis (TEI Crete/FORTH/IACM, Vassilika Vouton, GR-711 10 Heraklion, Crete, Greece, mixalis@iacm.forth.gr), Hanne Sagen (Nansen Environmental and Remote Sensing Center, Thormølensgt. 47, 5006 Bergen, Norway, hanne.sagen @nersc.no)

The Fram Strait is the main passage through which the mass and heat exchange between the Atlantic and the Arctic Ocean takes place. In the framework of DAMOCLES project (EU/FP6) a tomography experiment is planned to be conducted at latitude of 78N50 between 1E and 7E. A nearreal-time discrete inversion scheme has been developed relying on the matched-peak inversion approach seeking to maximize agreement between theoretical and observed peaks. The model relations between model parameters (temperature distributions) and travel times are implemented using a ray-tracing code accounting for the effects of range dependence. The parameter space is discretized and the corresponding model relations are stored in a database for later use - stored model relations greatly accelerate the inversion process. In the absence of real measurements the performance of the inversion scheme was tested using synthetic data relying on 3-year long time series of temperature data. The particular propagation conditions give rise to different inversion errors at different depths, with the minimum obtained at $250 \mathrm{~m}$ depth, in the core of the northward flowing Atlantic water. For the implementation of the tomographic analysis a software system called SMTAS has been developed running in a dedicated server connected to the internet.

MONDAY AFTERNOON, 30 JUNE 2008

ROOM 342A, 4:20 TO 7:20 P.M.

\section{Session 1pAOb}

\section{Acoustical Oceanography and ECUA: Marine Ecosystem Acoustics I}

\author{
Kenneth G. Foote, Cochair \\ Woods Hole Oceanographic Institution, Woods Hole, MA 02543, USA \\ Olav R. Godoe, Cochair \\ Institute of Marine Research, PO Box 1870, Bergen, 5817, Norway
}

Invited Papers

$4: 20$

1pAOb1. Ecosystem acoustics: meeting management needs. Olav Rune Godoe (Institute of Marine Research, C. Sundtsgt 64, Nordnes, 5817 Bergen, Norway, olavrune@imr.no)

Marine ecosystems and their exploited resources are often monitored through quasi-synoptic scientific surveys that produce estimates of population characteristics at periodic intervals. Spatial and temporal variability is an integral part of ecosystem function but is often ignored in traditional assessment methodology due to the inability of conventional surveys to resolve and quantify such effects. This confounds estimates of the state of the ecosystem and its main populations and obscures understanding of its dynamics and function. The concept and approach of ecosystem acoustics, which are elaborated and illustrated in this presentation, exploit both active and passive acoustics over their full bandwidth from a range of platforms giving temporal and spatial resolution sufficient for assessing 
the state of ecosystems including their main component organisms. Application of new platforms and sensors, as well as combinations of these, is enhancing the power of the approach. Ecosystem acoustics enables observations of marine life to be made on spatial and temporal scales relevant to understanding ecosystem function and assessment of system dynamics and state.

\section{4:40}

1pAOb2. Acoustical methods that provide an integrated view of the marine ecosystem. Anne Lebourges-Dhaussy (IRD, Technopole de la Pointe du Diable, BP 70, 29280 Plouzane, France, Anne.Lebourges.Dhaussy@ird.fr), Erwan Josse (IRD, Technopole de la Pointe du Diable, BP 70, 29280 Plouzane, France, Erwan.Josse@ird.fr)

The fish stock assessment are not anymore the sole purpose of the "fisheries acoustics" as it appears clearly that the fish belongs to a whole ecosystem, of which functioning mechanisms must be understood to better assess the fish populations' health state. The biotic factors, as prey availability, are of great importance and can be studied also by acoustic tools. The multifrequency systems (echosounders or profilers), increase the size range of the organisms that can be detected; new multibeam sonars provide a high resolution allowing studies close to boundaries. The results of the SIMFAMI European program are presented. It aimed at marine organism classification through multifrequency information, including fish/plankton separation and classification of meso/macroplankton. It provided new methods to classify and describe the various biotic components of the ecosystem from fish to plankton. A link between the use of these new methods and the former micro/mesoplankton classification tools as well as passive acoustic tools allows to reach the classification of a huge range of organisms and to study their interactions. Results of such experiences done on various upwelling ecosystems are shown. Additionally for complex fields, new multibeam sonars can advantageously help in the description of fish distributions.

5:00

1pAOb3. Three-dimensional structure of thin zooplankton layers is impacted by foraging fish. Kelly Benoit-Bird (Oregon State University, 104 COAS Admin Bldg, Corvallis, OR 97333, USA, kbenoit@coas.oregonstate.edu)

The role of predation by fish in the formation and persistence of zooplankton thin layers was assessed from three-dimensional observations using a multibeam sonar attached to a mechanical rotator. Zooplankton in Monterey Bay, CA, USA were found in intense layers with vertical scales of 0.5-2.5 m at night. These features comprised more than $90 \%$ of the zooplankton biomass and had volume scattering strengths of up to $-35 \mathrm{~dB}$. These thin zooplankton layers showed complex structure with significant though gradual undulations in their depth, thickness, and intensity. Fish spent significantly more time within zooplankton layers than was expected by chance, suggesting concentrated foraging activity. Sonar tracks of individual fish showed them diving through zooplankton layers. These dives were correlated with a decrease in the intensity of zooplankton scattering at the scale of tens of centimeters, resulting in the appearance of holes in the layer. Continued observation of layers revealed that these voids slowly filled in with zooplankton after the fish's departure. At the level of foraging observed, fish can have a significant effect on the distribution of zooplankton layers, however, the layers are resilient to apparent foraging by fish with reformation of the distinct layer often occurring post-predation.

\section{$5: 20$}

1pAOb4. Acoustic assessment of trophic dominance in a marine ecosystem. Richard E. Thorne (Prince William Sound Science Center, P.O. Box 705, Cordova, AK 99574, USA, rthorne@pwssc.org), Gary L. Thomas (University of Miami, RSMAS, 4600 Rickenbacker Causeway, Miami, FL 33149, USA, gthomas@rsmas.miami.edu)

High-frequency acoustic surveys over the past 15 years show that the trophic structure in Prince William Sound (PWS), Alaska, functions as a wasp-waist ecosystem. Three dominant biomasses in PWS are: (1) Pacific herring (2) walleye pollock and (3) the largebodied copepods (Neocalanus spps.) that dominate the spring zooplankton assemblage. The acoustic surveys and associated ecosystem observations suggest that the relative dominance of herring and pollock affects the composition of the apex predators. The near-shore and near-surface distribution of herring provides access by surface-oriented marine mammal and seabird predators, such as sea lions, seals, murres and cormorants, to a crucial winter-period food source. In contrast, the deep, off-shore distribution of the pollock favors large benthic predators, such as demersal sharks, halibut, and flounder. The acoustic surveys also demonstrated that the abundance of large-bodied copepods in PWS is critical to ecosystem productivity, including survival of juvenile pink salmon, and may affect the relative dominance of herring and pollock. An additional outcome of the long-term database on Pacific herring was the discovery of linkages between the 1989 Exxon Valdez oil spill and a subsequent collapse of the herring along with associated predators.

\section{5:40}

1pAOb5. Saguenay fjord entrance whale feeding ground: Acoustic study of sill dynamics and tidal aggregation of forage fish. Yvan Simard (Fisheries and Oceans Canada \& ISMER-UQAR, 850 route de la Mer, P.O. Box 1000, Mont-Joli, QC G5H-3Z4, Canada, simardy@dfo-mpo.gc.ca), Nathalie Roy (Fisheries and Oceans Canada \& ISMER-UQAR, 850 route de la Mer, P.O. Box 1000, MontJoli, QC G5H-3Z4, Canada, royn@dfo-mpo.gc.ca), François Saucier (Marine Sciences Institute, University of Québec, 310 Allée des Ursulines, P.O. Box. 3300, Rimouski, QC G5L-3A1, Canada, francois_saucier@uqar.qc.ca), Jacques Gagné (Fisheries and Oceans Canada, 850 route de la Mer, P.O. Box 1000, Mont-Joli, QC G5H-3Z4, Canada, gagneja@dfo-mpo.gc.ca), Samuel Giard (Fisheries and Oceans Canada, 850 route de la Mer, P.O. Box 1000, Mont-Joli, QC G5H-3Z4, Canada, giards@dfo-mpo.gc.ca)

The Saguenay fjord entrance in the St. Lawrence Estuary at Tadoussac is a world famous site where beluga and minke whales can be regularly observed from the coast. Strong tidal upwelling over the shallow sill of the fjord controls the exchanges with the St. Lawrence. In May 2005, an intensive oceanographic and acoustic survey was conducted to understand how the complex 3D hydrodynamics may contribute to concentrating whale preys. High time-space resolution acoustics (38 and $120 \mathrm{kHz}$ split-beam), ADCP (acoustic Doppler current profilers), CTD profiles, plankton and micronekton sampling were used to track the 3D movements of water masses, zooplankton and forage fish. During flood, the dense cold waters that jump over the sill block the Saguenay outflow and subduct into the fjord with their fish and zooplankton content. This complex 3D circulation generates fronts and convergence zones where 
biomass is concentrating until current reversal occurs during ebb. These concentrations are then advected and dispersed downstream over the sill with the Saguenay surface plume. This whale prey tidal concentrating process depends on the existence of sufficient forage fish densities in the mesoscale neighbourhood. It is likely at the basis of the persistent high frequentation of this area by whales.

\section{6:00}

1pAOb6. Multibeam sonar and echosounder to study schooling behaviour and predator-prey interactions of marine animals. Leif Nøttestad (Institute of Marine Research, Distribution and Trophic Interactions, P.O. Box 1870 Nordnes, N-5817 Bergen, Norway, leif.nottestad@imr.no)

In the past the technological developments and improved spatial and temporal resolution in hydro-acoustics, have made it possible to study schooling and aggregation behaviour of fish and predator-prey interactions in considerable detail. Hydro-acoustic methodology have thus revealed new and interesting scientific findings on animal behaviour and ecology previously difficult to observe and document from the marine environment. A fundamental challenge is still to be able to track individual fish within a school or animal aggregation at the same time as we observe the collective movements. The spatial resolution of hydro-acoustics is usually not sufficient enough to track individual fish within dense aggregations. Underlying mechanisms and motivations of individual animals swimming in aggregations are needed, in order to improve our understanding and predictability of collective behaviour. Improved post-processing programs for quantitative analysis of multi-beam sonar data are also needed. High-resolution underwater cameras, Crittercam-technology and individual tagging will offer complementary data sources to our understanding of detailed behaviour of marine animals. I will present synoptic ecological studies using multi-beam sonar and echosounder on schooling behaviour and predator-prey interactions including fish, seabirds and marine mammals.

\section{6:20}

1pAOb7. Acoustic characteristics of vertically migrating and non-migrating organisms observed in the North Pacific Ocean. Kouichi Sawada (Nat. Res. Inst. Fish. Engine., FRA, 7620-7 Hasaki, 314-0408 Kamisu, Japan, ksawada@ fra.affrc.go.jp), Hiroki Yasuma (Hokkaido Univ., 3-1-1 Minato-machi, 041-8611 Hakodate, Japan, ANB52615@ nifty.com), Taro Ichii (Nat. Res. Inst. Fish. Sci., FRA, 2-12-4 Fukuura, Kanazawa, 235-8648 Yokohama, Japan, ichii@ fra.affrc.go.jp), Hideyuki Takahashi (Nat. Res. Inst. Fish. Engine., FRA, 7620-7 Hasaki, 314-0408 Kamisu, Japan, hideyuki@fra.affrc.go.jp), Koki Abe (Nat. Res. Inst. Fish. Engine., FRA, 7620-7 Hasaki, 314-0408 Kamisu, Japan, abec@fra.affrc.go.jp)

Acoustic characteristics of vertically migrating and non-migrating organisms in a day were investigated for species identification. In July 27-30, 2004, both organisms were observed using ship echosounders ( $38 \mathrm{kHz}$ and $120 \mathrm{kHz}$ ) and a tethered acoustic-optical composite system (J-QUEST) from several hours before sunset to several hours after the sunrise, drifting a research vessel, Shunyo-maru, in the North Pacific Ocean. The J-QUEST is an instrumental package composed of an split-beam echosounder $(70 \mathrm{kHz})$ and a stereo TV camera system. The J-QUEST depth was changed from $220 \mathrm{~m}$ to $25 \mathrm{~m}$ to observe vertical migration. Boreopacific gonate squids were observed by the stereo TV camera and they are considered to conduct diurnal vertical migration to feed migrating organisms. Average TS of the ascending layer and Boreopacific squid were measured in situ using the J-QUEST. Average area scattering strength (SA) values of the ascending layer, non-migrating layer, descending layer, aggregation of Boreo pacific squids, and that of Japanee anchovy were measured at $38 \mathrm{kHz}$ and $120 \mathrm{kHz}$, There was a constant and different SA difference between the two frequencies at each layer except migrating layer. The ascending and the descending layer have almost the same SA differences. It indicates that the both layers composed of the same organisms.

\section{6:40}

1pAOb8. Acoustic GIS-based monitoring of Atlantic cod ecosystems in coastal Newfoundland. George A. Rose (Memorial Univ. of Newfoundland, Marine Inst., 155 Ridge Rd., St. John’s, NL A1C5R3, Canada, grose@mi.mun.ca)

Several bays in Newfoundland hold the largest extent groups of overwintering and spawning Atlantic cod (Gadus morhua), and are spawning areas for capelin (Mallotus villosus), the most important forage species. These species co-exist in coastal ecosystems whose physical features and ecological sensitivities restrict monitoring using conventional fisheries methods. Active and passive acoustic methods, acoustic telemetry, oceanographic instrumentation and ROV video have been used to monitor overwintering and spawning distributions and abundance of cod, using mobile and fixed platforms. Cod behaviour is complex and features high mobility both horizontally and vertically, especially during migratory and spawning periods. Overwintering cod have school packing densities $>>1$ fish. $\mathrm{m}^{-3}$. Spawning features increased mobility and vertical structures or "columns" of individual fish and sound production captured using stationary hydrophones. Acoustic returns from aquatic vegetation and bottom types have been used to map juvenile habitat. Acoustic telemetry has established the movement patterns of male and female fish during spawning and the homing characteristics of cod as the basis of their stock structure. The movements and spawning behaviour of capelin can also be monitored as can interactions between predators and prey. An experiment using real-time and 3D location telemetry in a comprehensive GIS system will be described. 
1pAOb9. Using multifrequency acoustic scattering techniques to study mixed zooplankton populations. Andone Lavery (Woods Hole Oceanographic Institution, Applied Ocean Physics \& Engineering Department, 98 Water Street, MS \#11, Woods Hole, MA 02543, USA, alavery@whoi.edu), Peter Wiebe (Woods Hole Oceanographic Institution, Biology Department, 45 Water Street, MS \#33, Woods Hole, MA 02543, USA, pwiebe@whoi.edu), Timothy K. Stanton (Woods Hole Oceanographic Institution, Applied Ocean Physics \& Engineering Department, 98 Water Street, MS \#11, Woods Hole, MA 02543, USA, tstanton@whoi.edu), Gareth Lawson (Stanford University, Hopkins Marine Station, 120 Oceanview Blvd, Pacific Grove, CA 93950, USA, glawson@stanford.edu), Mark Benfield (Louisiana State University, Department of Oceanography \& Coastal Sciences, 2179 Energy, Coast \& Environment Building, Baton Rouge, LA 70803, USA, mbenfie@1su.edu), Nancy Copley (Woods Hole Oceanographic Institution, Biology Department, 45 Water Street, MS \#33, Woods Hole, MA 02543, USA, ncopley@whoi.edu)

Zooplankton populations can be highly heterogeneous and variable on spatial scales of centimeters to kilometers and temporal scales of minutes to years. High-frequency acoustic scattering techniques allow a synoptic view of zooplankton distribution and abundance on these scales. However, there are well-known complications in the interpretation of acoustic scattering returns, often limiting the inference of quantitative parameters (such as size or abundance) to restricted locations and conditions. Multifrequency and broadband acoustic scattering techniques, combined with sophisticated instrument platforms that combine multiple sensors allowing acoustical, optical, and other environmental measurements, reduce the ambiguities typically associated to the interpretation of acoustic scattering at single frequencies and expand the conditions under which inference of quantitative biological parameters is possible. The focus of this talk is a decade-long study aimed at understanding the distribution and abundance of mixed zooplankton populations in the Gulf of Maine. Simultaneous multifrequency acoustic, optical, environmental, and net measurements were performed. Insights gained from this study are presented together with recent measurements involving broadband acoustic scattering from zooplankton in the presence of nonlinear internal solitary waves. These studies highlight the importance of an integrated approach to understanding heterogeneous zooplankton populations on relevant spatial and temporal scales.

MONDAY AFTERNOON, 30 JUNE 2008

ROOM 352B, 1:00 TO 5:20 P.M.

\title{
Session 1pBBa
}

\section{Biomedical Ultrasound/Bioresponse to Vibration: High-Intensity Focused Ultrasound I}

\author{
Lawrence A. Crum, Cochair \\ Center for Industrial and Medical Ultrasound, Applied Physics Lab., University of Washington, 1013 NE 40th St., \\ Seattle, WA 98105, USA \\ Jean Yves Chapelon, Cochair \\ INSERM, U556, 151 Cours Albert Thomas, Lyon, 69003, France
}

\section{Invited Papers}

\section{1:00}

1pBBa1. The feasibility of local harmonic motion imaging for the guidance and control of focused ultrasound surgery. Kullervo Hynynen (University of Toronto, Sunnybrook Health Sciences Centre, Suite S6 65b, 2075 Bayview Ave, Toronto, ON M4N 3M5, Canada, khynynen@sri.utoronto.ca), Laura Curiel (University of Toronto, Sunnybrook Health Sciences Centre, Suite S6 65b, 2075 Bayview Ave, Toronto, ON M4N 3M5, Canada, lcuriel@sri.utoronto.ca), Rajiv Chopra (University of Toronto, Sunnybrook Health Sciences Centre, Suite S6 65b, 2075 Bayview Ave, Toronto, ON M4N 3M5, Canada, chopra@sri.utoronto.ca)

A local harmonic motion (LHM) can be generated within the tissues by the periodic induction of radiation force using a focused ultrasound (FUS) transducer. Tissue motion can then be tracked by collecting RF signals during the excitation using a separate transducer. Finally, displacement estimates can be obtained by cross-correlating the collected RF signals. The characteristics of the induced LHM depend on the local elastic properties of the tissues making it an attractive tool for imaging and therapy control applications. LHM measurements have been obtained in vivo on rabbit muscle and it was observed that the amplitude of the motion was significantly reduced after coagulation. LHM was successfully used to spatially detect the presence of the coagulation lesions within the tissues as a drop in LHM amplitude. It was also possible to detect the location of an implanted VX2 tumor when a spatial scan was performed as the LHM amplitude was lower inside the tumor because of an increased stiffness. Measurements of LHM during tissue heating using FUS reflected the changes in stiffness and revealed the apparition of coagulation showing the potential of these measurements as an alternative control for the FUS exposure. [Work supported by NIH GrantR33 CA102884 and CRC.] 
1pBBa2. Clinical experiences with extracorporeal Ultrasound-guided high-intensity focused ultrasound treatment for cancer patients. Feng Wu (Chongqing Medical University, Institute of Ultrasound Engineering in Medicine \& Clinical Centre for Tumour Therapy, 1 Medical College Road, 400016 Chongqing, China, mfengwu@yahoo.com)

Noninvasive, image-guided tumour thermal ablation with extracorporeal high-intensity focused ultrasound (HIFU) has received increasing interest in the treatment of patients with solid tumours. Since December 1997, an extracorporeal ultrasound-guided HIFU system (Mode-JC, Haifu Technology Co. Ltd., Chongqing, China) has been used to treat approximately 10,000 patients with solid tumours in China, including those of liver, breast, bone, kidney, pancreas, soft tissue, and uterus. The same device has been recently introduced into the UK, Italy, Japan, and South Korea, and so far, more than 1,000 patients have received HIFU treatment outside China. The purpose of this article is to introduce our clinical experiences using extracorporeal, ultrasound-guided HIFU ablation for solid tumours. Five-year follow-up data are observed in patients with primary liver cancer, breast cancer, and osteosarcoma. Among patients treated with HIFU, an extremely low major complication rate is observed. In conclusion, our clinical studies indicate that HIFU treatment is a safe, effective, and feasible modality in the treatment of cancer patients.

\section{$1: 40$}

1pBBa3. Adaptive focusing for bone and motion artifacts corrections in High Intensity Focused Ultrasound Therapy. Mickael Tanter (Laboratoire Ondes et Acoustique, ESPCI, Université Paris 7, CNRS, 10 rue Vauquelin, 75005 Paris, France, michael.tanter@espci.fr), Mathieu Pernot (Laboratoire Ondes et Acoustique, ESPCI, Université Paris 7, CNRS, 10 rue Vauquelin, 75005 Paris, France, mathieu.pernot@espci.fr), Jean-François Aubry (Laboratoire Ondes et Acoustique, ESPCI, Université Paris 7 , CNRS, 10 rue Vauquelin, 75005 Paris, France, jean-francois.aubry@espci.fr), Mathias Fink (Laboratoire Ondes et Acoustique, ESPCI, Université Paris 7, CNRS, 10 rue Vauquelin, 75005 Paris, France, mathias.fink@espci.fr)

Despite extensive and fast progress of HIFU clinical applications, many issues still need to be addressed. Distortions caused by defocusing obstacles, such as the skull or ribs, on the ultrasonic therapeutic beam are still being investigated. Multi-element transducer technology must be used in order to achieve such transcranial or transcostal adaptive focusing. Second, the problem of motion artifacts, a key component in the treatment of abdominal lesions, has been shown to significantly influence the efficacy and treatment time. Though many methods have been proposed for the detection of organ motion, little work has been done to develop a comprehensive solution including motion tracking and feedback correction in real time. This paper is a review of the work achieved by authors in transcranial HIFU, transcostal HIFU, and motion compensated HIFU. For these three issues, the optimal solution can be reached using the same technology of multi-element transducers devices able to work both in Transmit and Receive modes.

\section{Contributed Papers}

\section{2:00}

1pBBa4. Using a toroid transducer for thermal ablation by high-intensity-focused ultrasound increases the coagulated volume. David Melodelima (INSERM, U556, 151 Cours Albert Thomas, 69003 Lyon, France, melodelima@lyon.inserm.fr), William N'Djin (INSERM, U556, 151 Cours Albert Thomas, 69003 Lyon, France, ndjin@lyon.inserm.fr), Hubert Parmentier (INSERM, U556, 151 Cours Albert Thomas, 69003 Lyon, France, hubert.parmentier@chu-lyon.fr), Michel Rivoire (Institut de Chirurgie Experimentale - Centre Léon Berard, 28 rue Laennec, 69008 Lyon, France, rivoire@lyon.fnclcc.fr), Jean Yves Chapelon (INSERM, U556, 151 Cours Albert Thomas, 69003 Lyon, France, chapelon@1yon.inserm.fr)

Here, we report that a new design of High Intensity Focused Ultrasound transducer can significantly enlarge the coagulated volume over short periods of time, and that treatment in the liver can be guided in real-time using an integrated ultrasound imaging probe. Eight ultrasound emitters, divided into 256 elements, were created by sectioning a single toroid piezocomposite transducer. The focal zone was conical in shape and located $70 \mathrm{~mm}$ from the transducer; enabling the treatment of deep-seated tumors. A single thermal lesion was created when the eight emitters performed alternative and consecutive $5 \mathrm{~s}$ ultrasound exposures. This paper presents in vivo evidence that the coagulated volume obtained from a $40 \mathrm{~s}$ total exposure in the liver was $7.0 \pm 2.5 \mathrm{~cm}^{3}\left(\min 1.5-\max 20.0 \mathrm{~cm}^{3}\right)$. All lesions were visible with high contrast on sonograms. The correlation between the diameter of lesions observed on sonograms and during gross examination was $92 \%$. This method also allowed the user to easily enlarge the coagulated volume by juxtaposing single lesions. This approach may have a role in treating unresectable colorectal liver metastases and may also be used in conjunction with resection to extend its limits.

\section{2:20}

1pBBa5. Temperature measurements and determination of cavitation thresholds during High Intensity Focused Ultrasound (HIFU) Exposures in ex-vivo porcine muscle. Subha Maruvada (U.S. Food and Drug Administration, 10903 New Hampshire Ave., Silver Spring, MD 20993, USA, subha.maruvada@fda.hhs.gov), Yunbo Liu (U.S. Food and Drug Administration, 10903 New Hampshire Ave., Silver Spring, MD 20993, USA, yunbo.liu@fda.hhs.gov), Bruce A. Herman (U.S. Food and Drug Administration, 10903 New Hampshire Ave., Silver Spring, MD 20993, USA, bruce.herman@fda.hhs.gov), William F. Pritchard (U.S. Food and Drug Administration, 10903 New Hampshire Ave., Silver Spring, MD 20993, USA, william.pritchard@fda.hhs.gov), Gerald R. Harris (U.S. Food and Drug Administration, 10903 New Hampshire Ave., Silver Spring, MD 20993, USA, gerald.harris@fda.hhs.gov)

Cavitation in HIFU procedures can yield unpredictable results, particularly when the same location is targeted for more than several seconds. To study this effect, temperature rise was measured in fresh ex-vivo porcine tissue during HIFU exposures. Immediately following euthanasia, a section of back muscle (latissimus dorsi) was resected and a $50 \mu \mathrm{m}$ diameter fine bare wire thermocouple was placed via needle through the tissue. $825 \mathrm{kHz}$ HIFU was then applied to the tissue focused at the thermocouple junction. Thirty second HIFU exposures of increasing pressure from 1-7.5 MPa were applied and the temperature rise and decay during and after sonication were recorded. B-mode imaging was used to monitor any cavitation activity during sonication. If cavitation was noted during the sonication, the sonication was repeated at the same pressure level two more times at 20 minute intervals in order to characterize the repeatability given that cavitation has occurred. The cavitation threshold of porcine muscle was determined to be between 4 and $7 \mathrm{MPa}$. Temperature traces obtained at various pressure 
levels demonstrated a wide range of heating profiles in fresh ex-vivo tissue due to both the occurrence of cavitation and viscous heating artifacts. (This research was supported by DARPA IAG \# 224-05-6016).

\section{2:40}

1pBBa6. Therapeutic ultrasound induced cell death from a histological perspective. Andrew Brayman (Center for Industrial and Medical Ultrasound, Applied Physics Lab., University of Washington, 1013 NE 40th St., Seattle, WA 98105, USA, brayman@apl.washington.edu), Peter Kaczkowski (Center for Industrial and Medical Ultrasound, Applied Physics Lab., University of Washington, 1013 NE 40th St., Seattle, WA 98105, USA, peter@apl.washington.edu), Yak-Nam Wang (Applied Physics Laboratory, University of Washington, $1013 \mathrm{NE}$ 40th Street, Seattle, WA 98105, USA, ynwang@apl.washington.edu), Marilee Andrew (Center for Industrial and Medical Ultrasound, Applied Physics Lab., University of Washington, 1013 NE 40th St., Seattle, WA 98105, USA, marilee@apl.washington.edu), Lawrence A. Crum (Center for Industrial and Medical Ultrasound, Applied Physics Lab., University of Washington, 1013 NE 40th St., Seattle, WA 98105, USA,
lac@apl.washington.edu),Steven Kargl(Applied Physics Laboratory, University of Washington, 1013 NE 40th Street, Seattle, WA 98105, USA, karg1@troutmask.apl.washington.edu), Gavriel Speyer (Center for Industrial and Medical Ultrasound, Applied Physics Lab., University of Washington, 1013 NE 40th St., Seattle, WA 98105, USA, gavriel@u.washington .edu)

High-power, short-exposure time, High Intensity Focused Ultrasound (HIFU) treatment protocols are under development that offer the potential to increase procedure throughput and optimize individual therapies. Histological examination and optical image analysis of tissues following dynamic HIFU exposure in ex vivo bovine liver have revealed that cells undergo a fundamentally different form of cell death. The rapid temperature rise due to the HIFU exposure leaves the cells structurally intact but no longer viable, similar to the cell "fixation" induced by snap-freezing. These results suggest that careful choice of both staining technique and metric for determining cell death are important in quantifying type and morphology of cell ablation, and more broadly, safety and efficacy of treatment. This finding is similar to those obtained and under discussion in the laser and RF ablation communities. Specifically, the NADH staining technique is superior to H\&E for assessing cell viability, and an alternative measure of cell death may be preferable to the binary thermal dose threshold currently the standard for HIFU treatment. [Work supported by NIH.]

\section{Invited Papers}

\section{3:00}

1pBBa7. Ultrasound interstitial applicators for thermal ablation in liver. Cyril Lafon (INSERM, U556, 151 Cours Albert Thomas, 69003 Lyon, France, lafon@lyon.inserm.fr), Rares Salomir (INSERM, U556, 151 Cours Albert Thomas, 69003 Lyon, France, salomir@lyon.inserm.fr), Guillaume Bouchoux (INSERM, U556, 151 Cours Albert Thomas, 69003 Lyon, France, bouchoux@1yon.inserm.fr), Alain Birer (INSERM, U556, 151 Cours Albert Thomas, 69003 Lyon, France, birer@lyon.inserm.fr), Neil Owen (INSERM, U556, 151 Cours Albert Thomas, 69003 Lyon, France, Neil.Owen@lyon.inserm.fr), Eric Delabrousse (INSERM, U556, 151 Cours Albert Thomas, 69003 Lyon, France, delabrousse@lyon.inserm.fr), François Mithieux (INSERM, U556, 151 Cours Albert Thomas, 69003 Lyon, France, mithieux@lyon.fnclcc.fr), Jean Yves Chapelon (INSERM, U556, 151 Cours Albert Thomas, 69003 Lyon, France, chapelon@1yon.inserm.fr)

Aggressive treatment of localized hepatic metastases, by surgery or other means, was proven to be a viable strategy for improving the prognoses of many patients. In that context, thermal ablation by high intensity ultrasound was proposed and used in clinics. However, for treating deep-seated tumors and in most cases, radiofrequency and cryotherapy probes are applied interstitially. Interstitial ultrasound applicators were proposed as an intermediate solution. The treatment can be focused, deeper than with other physical agents, and the transducer can eventually both treat and image tissues. In our experience, two approaches were investigated: percutaneous and intratissular, or endo vascular. The active element was a miniature flat transducer operating at a frequency of $5 \mathrm{MHz}$, for a satisfactory tradeoff between beam penetration and energy absorption. In vivo trials on a porcine model demonstrated that both procedures are minimally invasive and that large thermal lesions, up to $20 \mathrm{~mm}$ deep, can be obtained. Technological improvements such as the use of dual mode transducers (for imaging and therapy) or the performance under MRI guidance allowed monitoring the treatment in real-time.

$$
3: 20
$$

1pBBa8. Histotripsy and the developing role of microbubbles in ultrasound therapy. Jeffrey B. Fowlkes (University of Michigan, Department of Radiology, Kresge III, R3320, Ann Arbor, MI 48109-0553, USA, fowlkes@umich.edu)

Microbubbles in therapeutic ultrasound has seen increasing interest in recent years. Once thought to be problematic, bubble production and interactions with ultrasound fields are yielding promising new methods and this presentation will review two areas of collaborative research at the University of Michigan investigating therapeutic applications utilizing microbubbles. Histotripsy uses short pulses of high intensity ultrasound produce cavitation in vivo and subdivide tissue at a subcellular level. The highly localized bubble activity homogenizes tissue to the point of reducing acoustic backscatter enabling lesion detection. Lesion margins are remarkably fine, even yielding fractional disruption of cells. The homogenized material is readily absorbed thus debulking tissue with little residual scar. At the other end of the acoustic intensity scale, microbubbles can be produced by triggering vaporization of superheated liquid droplets, termed acoustic droplet vaporization (ADV). ADV requires diagnostic levels of ultrasound to yield localized microbubbles production. The method is being investigated for direct occlusion therapy and perfusion control to augment other therapeutic methods including drug delivery among other potential applications. These methods highlight the growing field of microbubble-based therapies, which will expand with our increasing understanding of microbubbles and their interactions with surrounding environments. Supported in part by NIH EB00281, HL077629 and EB006476.

3:40-4:00 Break 


\section{Contributed Papers}

\section{4:00}

1pBBa9. Localization and enhancement of cavitation and heating during HIFU exposure using microparticles of high surface roughness. Manish Arora (University of Oxford, Medical Engineering Unit, 43 Banbury Road, OX2 6PE Oxford, UK, manish.arora@eng.ox.ac.uk), Costas Arvanitis (University of Oxford, Medical Engineering Unit, 43 Banbury Road, OX2 6PE Oxford, UK, costas.arvanitis@eng.ox.ac.uk), Emma Cox (University of Oxford, Medical Engineering Unit, 43 Banbury Road, OX2 6PE Oxford, UK, eflcox@gmail.com), Constantin C. Coussios (University of Oxford, Medical Engineering Unit, 43 Banbury Road, OX2 6PE Oxford, UK, constantin.coussios@eng.ox.ac.uk)

The occurrence of cavitation during HIFU exposure is associated with higher heating rates than in the absence of bubble activity. However, the peak rarefaction pressures required to initiate cavitation in vivo are high (7-10 MPa), making it difficult to localize, sustain, and confine cavitation activity and its associated bioeffects. In this work, we explore the use of rough microparticles to promote and localize cavitation nucleation during HIFU exposures. Polystyrene microparticles of mean diameter $\sim 2 \mu \mathrm{m}$ are embedded in a polyacrylamide tissue mimicking phantom, which has a high cavitation threshold similar to tissue. The temperature rise during HIFU exposure is measured using an embedded needle thermocouple, whilst cavitation activity is simultaneously monitored using a passive cavitation detector (PCD). The presence of particles is shown to lower the cavitation threshold significantly and, above this threshold, to result in higher heating rates than in the absence of particles for the same focal intensity. Furthermore, it is found that particles make it possible to repeatedly initiate cavitation at the same location and lead to increased broadband noise emissions. Finally, a strong correlation is found between enhanced heating and the broadband emissions arising from inertial cavitation.

\section{4:20}

1pBBa10. Local heating by a bubble excited by high intensity focused ultrasound. Wayne Kreider (Center for Industrial and Medical Ultrasound, Applied Physics Lab., University of Washington, 1013 NE 40th St., Seattle, WA 98105, USA, wkreider@u.washington.edu), Michael S. Canney (Center for Industrial and Medical Ultrasound, Applied Physics Lab., University of Washington, 1013 NE 40th St., Seattle, WA 98105, USA, mcanney@u.washington.edu), Michael R. Bailey (Center for Industrial and Medical Ultrasound, Applied Physics Lab., University of Washington, 1013 NE 40th St., Seattle, WA 98105, USA, bailey@apl.washington.edu), Vera A. Khokhlova (Center for Industrial and Medical Ultrasound, Applied Physics Lab., University of Washington, 1013 NE 40th St., Seattle, WA 98105, USA, vera@acs366.phys.msu.ru), Lawrence A. Crum (Center for Industrial and Medical Ultrasound, Applied Physics Lab., University of Washington, 1013 NE 40th St., Seattle, WA 98105, USA, lac@apl .washington.edu)

A current topic of interest for high intensity focused ultrasound (HIFU) treatments involves the relative roles of bubbles and nonlinear acoustic propagation as heating mechanisms. At high amplitudes, nonlinear propagation leads to the generation of boiling bubbles within milliseconds; at lower amplitudes, cavitation bubbles can enhance heating through viscous dissipation, acoustic radiation, and heat conduction. In this context, understanding the physics attendant to HIFU bubbles requires consideration of gas-vapor bubble dynamics, including thermal effects in the nearby liquid. To this end, recent experimental observations with high-speed photography suggest that bubbles undergo a brief period of growth after application of HIFU has stopped. To explain this observation, a model is implemented that couples the thermodynamic state of a strongly driven bubble with thermal conditions in the surrounding liquid. From model simulations, liquid heating in the vicinity of a HIFU bubble is estimated. Calculations suggest that thermal conduction and viscous dissipation can lead to the evolution of a nontrivial thermal boundary layer. Development of a boundary layer that reaches superheated temperatures would explain the aforementioned experimental observation. As such, cavitation bubbles and boiling bubbles share important characteristics during HIFU. [Work supported by NIH DK43881 and NSBRI SMS00402.]

4:40

1pBBa11. Temperature and size-dependence of the vaporization threshold of phase-shift emulsions. Tyrone Porter (Boston University, 110 Cummington Street, Boston, MA 02215, USA, tmp@bu.edu), Peng Zhang (Boston University, 110 Cummington Street, Boston, MA 02215, USA, pzhang@bu.edu)

Acoustic cavitation has proven to be important for several therapeutic applications of ultrasound. However, acoustic cavitation is difficult to initiate and sustain in the absence of cavitation nuclei, particularly in tissue. Phase shift emulsions are ideal candidates for cavitation nuclei for in vivo applications. These emulsions, which consist of superheated liquid perfluorocarbon droplets enclosed by albumin shells, may be vaporized with acoustic pulses, a process known as acoustic droplet vaporization (ADV). In this study, we determined the ADV threshold at $2 \mathrm{MHz}$ as a function of temperature and droplet size. Studies were conducted with micro- and nanosized emulsions in a closed-flow system immersed in a temperature controlled water bath. The emulsions were injected into a flow system and exposed to high intensity focused ultrasound (HIFU; acoustic parameters: 2 $\mathrm{MHz}, 10$ cycles, $100 \mathrm{~Hz}$ pulse repetition frequency). A portable diagnostic ultrasound scanner was used to monitor for vaporization. Upon vaporization, the peak rarefactional pressure of the acoustic pulse and the water bath temperature were recorded. It was determined that the vaporization threshold was independent of droplet size and inversely proportional to temperature. The utility of these emulsions in cancer therapy, particularly for bubbleenhanced heating during HIFU exposure, will be discussed.

\section{5:00}

1pBBa12. In vitro evaluation of an oscillating 5-element dual-mode transducer. Neil Owen (INSERM, U556, 151 Cours Albert Thomas, 69003 Lyon, France, Neil.Owen@lyon.inserm.fr), Guillaume Bouchoux (INSERM, U556, 151 Cours Albert Thomas, 69003 Lyon, France, bouchoux@lyon.inserm.fr), Alain Birer (INSERM, U556, 151 Cours Albert Thomas, 69003 Lyon, France, birer@lyon.inserm.fr), Rémi Berriet (Imasonic, Z.A. rue des Savourots, 70190 Voray sur l'Ognon, France, Remi.Berriet@imasonic.com), Jean Yves Chapelon (INSERM, U556, 151 Cours Albert Thomas, 69003 Lyon, France, chapelon@lyon.inserm.fr), Gérard Fleury (Imasonic, 15 rue Alain Savary, 25000 Besançon, France, Gerard.Fleury@imasonic.com), Cyril Lafon (INSERM, U556, 151 Cours Albert Thomas, 69003 Lyon, France, lafon@lyon.inserm.fr)

Miniature dual-mode transducers can be used for minimally invasive treatment of deep-seated tumors. While in contact with the tissue, the transducer guides and monitors localized necrosis. Here, an oscillating 5-element piezo-composite transducer was characterized, and then evaluated in vitro using porcine liver. Each element was $3.0 \times 3.8 \mathrm{~mm}^{2}$ with a geometric cylindrical focus of $14 \mathrm{~mm}$. The transmit frequency was determined by the maximal electro-acoustic efficiency, $65 \%$, which was found at $5.6 \mathrm{MHz}$. The transmit-receive impulse response was $400 \mathrm{~ns}$ long at $-6 \mathrm{~dB}$, and the $-6 \mathrm{~dB}$ fractional bandwidth, centered at $5.6 \mathrm{MHz}$, was $30 \%$. Axial and lateral resolution measured with a $0.1 \mathrm{~mm}$ diameter wire was $0.5 \mathrm{~mm}$ and $2.0 \mathrm{~mm}$, respectively. For therapy, all elements radiated simultaneously, and for imaging, independently. Treatment was performed at increments of $20^{\circ}$ to form a composite volume of necrosis. At each angle, ultrasound was applied for $60 \mathrm{~s}$ at a transducer surface intensity of $15 \mathrm{~W} / \mathrm{cm}^{2}$. Pulse-echo data were recorded while the transducer oscillated over a $180^{\circ}$ sector to form images before and after treatment at each angle. Gross examination of lesion size agreed well with echogenic region size in the images. [Supported by ANR and Inserm Post-doctoral Fellowship.] 


\title{
Session 1pBBb
}

\section{Biomedical Ultrasound/Bioresponse to Vibration: Quantitative Ultrasound Methods for Diagnosis and Therapy I}

\author{
Jonathan Mamou, Cochair \\ Riverside Research Institute, 156 William St., 9th Floor, New York, NY 10038, USA \\ Geneviève Berger, Cochair \\ Université Paris 6, Laboratoire d'Imagerie Paramétrique, 15 rue de l'Ecole de médecine, Paris, France
}

\section{Invited Papers}

1:00

1pBBb1. Techniques and modalities for high resolution imaging and assessment of skin with high-frequency ultrasound (20 to $100 \mathrm{MHz}$ ). Michael Vogt (Institute of High-Frequency Engineering, Ruhr-University Bochum, IC 6/133, 44780 Bochum, Germany, Michael.Vogt@rub.de), Helmut Ermert (Institute of High-Frequency Engineering, Ruhr-University Bochum, IC 6/133, 44780 Bochum, Germany, Helmut.Ermert@rub.de)

High-frequency ultrasound (HFUS) in the $20 \mathrm{MHz}$ range is routinely used in dermatological diagnosis for morphological skin imaging in order to support the identification of suspicious skin lesions and to evaluate the success of therapies. In this presentation, technical developments for the utilization of higher ultrasound frequencies in the range up to $100 \mathrm{MHz}$ are presented, which allow for high-resolution imaging and assessment of skin structures with an axial resolution down to $10 \mu \mathrm{m}$. Furthermore, a new HFUS system for the assessment of elastic skin properties and quantitative strain imaging with $20 \mathrm{MHz}$ ultrasound is presented as a new imaging modality. Also, a reflection-tomography skin imaging concept was implemented with a $20 \mathrm{MHz}$ ultrasound limited-angle spatial compound system, which allows for multi-directional analysis of backscattered and reflected ultrasound waves from different insonation angles. The proposed techniques and modalities have been evaluated by measurements on phantoms and in vivo. Results show that high-resolution qualitative and quantitative skin assessment methods have been successfully implemented. HFUS based strain imaging enables the non-invasive assessment of skin and lesion properties, and HFUS limited-angle spatial compounding allows for improved skin analysis compared to the conventional linear-scan imaging approach.

$$
1: 20
$$

1pBBb2. Quantitative intravascular ultrasound elasticity imaging as an imaging biomarker in clinical trials. Ton Van Der Steen (Thorax centre ErasmusMC, Ee23.02, pobox 2040, 3000 CA Rotterdam, Netherlands, a.vandersteen@erasmusmc.nl), Johannes A. Schaar (Biomedical Engineering, Erasmus MC, P.O. Box 2040, 3000 CA Rotterdam, Netherlands, j.schaar@erasmusmc.nl), Frits Mastik (Biomedical Engineering, Erasmus MC, P.O. Box 2040, 3000 CA Rotterdam, Netherlands, f.mastik@erasmusmc.nl), Hector Garcia (Biomedical Engineering, Erasmus MC, P.O. Box 2040, 3000 CA Rotterdam, Netherlands, HGarcia@cardialysis.nl), Mike Danilouchkine (Biomedical Engineering, Erasmus MC, P.O. Box 2040, 3000 CA Rotterdam, Netherlands, m.danilouchkine@erasmusmc.nl), Radj Baldewsing (Biomedical Engineering, Erasmus MC, P.O. Box 2040, 3000 CA Rotterdam, Netherlands, radj.baldewsing@ @ewitt.com), Patrick W. Serruys (Biomedical Engineering, Erasmus MC, P.O. Box 2040, 3000 CA Rotterdam, Netherlands, p.w.j.c.Serruys@erasmusmc.nl)

The composition and morphology of an atherosclerotic lesion are currently considered more important determinants of acute coronary ischemic syndromes that the degree of stenosis. When a lesion is unstable, it can rupture and cause an acute thrombotic reaction. An unstable plaque can be characterized by a lipid core that is covered by a thin fibrous cap, which has been locally weakened by inflammatory cells. Intravascular Ultrasound Palpography is an intravascular ultrasound based technique that is capable to measure the local strain in coronaries and atherosclerotic plaque. This strain is induced by varying intraluminal pressure. This lecture will show principles of the technology and how this technology is used in clinical trials. Results from a trial with traditional lipid lowering treatment (IBIS1) and from a trial on the efficacy of a new medication (IBIS2) will be presented. Furthermore the potential of Intravascular Ultrasound Modulography will be discussed. This work is financially supported by the Dutch Technology Foundation, The Dutch Heart Foundation and a research grants from Volcano Corporation and Glaxo Smith Kline

\section{$1: 40$}

1pBBb3. Recent progress of acoustic microscopy for medicine and biology. Yoshifumi Saijo (Tohoku University, 4-1 Seiryomachi, Aoba-ku, 980-8575 Sendai, Japan, saijo@idac.tohoku.ac.jp), Kazuto Kobayashi (Honda Electronics Co. Ltd., 20 Oyamazuka, Oiwa-cho, 441-3193 Toyohashi, Japan, kazuto@honda-el.co.jp), Takahiro Iwamoto (Tohoku University, 4-1 Seiryomachi, Aoba-ku, 980-8575 Sendai, Japan, iwamoto@yoshizawa.ecei.tohoku.ac.jp), Nagaya Okada (Honda Electronics Co. Ltd., 20 Oyamazuka, Oiwacho, 441-3193 Toyohashi, Japan, nagaya@honda-el.co.jp), Akira Tanaka (Fukushima University, 1 Kanayagawa, 960-1296 Fukushima, Japan, a-tanaka@sss.fukushima-u.ac.jp), Naohiro Hozumi (Aichi Institute of Technology, 1247 Yachigusa, Yakusa-cho, 4700392 Toyota, Japan, hozumi@ aitech.ac.jp)

Dramatic shortening of the calculation time provided by the recent progress of the computer technology has made biomedical researchers possible to assess nonlinear acoustic phenomena in soft materials which had been assumed as acting linearly. Besides, the spread of Windows-based personal computer and peripheral devices enabled easier and cheaper configuration of the whole acoustic microscope system such as pulse generation, analogue/digital conversion, mechanical scanning and image processing. According to 
these progresses, conventional acoustic microscopy with only C-mode imaging has widened its data acquisition mode to B-mode, C-mode, surface acoustic impedance mode, and three-dimensional (3D) imaging. The base of our acoustic microscope system was consisted of (1) PVDF transducer with the central frequency of $100 \mathrm{MHz}$, (2) ultrasonic pulser made of high speed semiconductor switching, (3) mechanical scanner using two linear servo motors, (4) high speed PCI card digitizer with the sampling frequency of 2 $\mathrm{GHz}$, and (5) personal computer controlling the whole system. For skin imaging, 3D imaging of the fingerprint was reconstructed by consecutive B-mode imaging. Surface acoustic impedance imaging of the fingerprint can be obtained by just putting a finger on a thin plastic plate of the transducer. Conventional C-mode imaging of thinly sliced skin sample presented quantitative values of thickness, attenuation and sound speed of the tissue.

1pBBb4. Improved cancer detection and classification using multi-parameter quantitative ultrasound. Michael L. Oelze (University of Illinois at Urbana-Champaign, Beckman Institute, 405 N Mathews, Urbana, IL 61801, USA, oelze@uiuc.edu), William D. O’Brien, Jr. (University of Illinois at Urbana-Champaign, Beckman Institute, 405 N Mathews, Urbana, IL 61801, USA, wdo@uiuc .edu)

Quantitative ultrasound (QUS) has been used successfully to differentiate benign from malignant solid tumors in animal models of breast cancer. In these studies, QUS made use of estimates of average scatterer diameter (ASD) and average acoustic concentration (AAC) from the ultrasonic backscatter. However, initial attempts to classify different kinds of malignant tumors based on ASD and AAC estimates were not successful. New models for ultrasonic backscatter were created by considering the cytoskeletal structure of cells. In addition, the homodyned $\mathrm{K}$ distribution was used to model the amplitude of the envelope from regions-of-interest in the malignant tumors. The homodyned $\mathrm{K}$ distribution yielded two parameters: the S parameter, which quantified the randomness of scatterer spacings, and the $\beta$ parameter, which quantified the amount of clustering of scatterers in the interrogated tissue. Statistically significant differences $(\mathrm{P}<0.05)$ were observed between average $\mathrm{S}$ and $\beta$ parameters from the malignant tumors. Furthermore, statistically significant differences $(\mathrm{P}<0.05)$ were observed between ASD and AAC estimates from the malignant tumors using the new scattering models at ultrasonic frequencies above $16 \mathrm{MHz}$. The use of four parameters, as opposed to two, improved the ability to uniquely classify different kinds of malignant tumors. [Supported by NIH R01CA111289.]

1pBBb5. Modeling scattering from cells and biological structures. Michael C. Kolios (Ryerson University, Department of Physics, 350 Victoria Street, Toronto, ON M5B2K3, Canada, mkolios@ryerson.ca), Gregory J. Czarnota (Sunnybrook Health Sciences Centre, Department of Radiation Oncology and Imaging Research, 2075 Bayview Ave, T2-Wing, Toronto, AB M4N 3M5, Canada, Gregory.Czarnota@sunnybrook.ca)

The goal of ultrasonic tissue characterization is to extract information over and above the information conventionally displayed on imaging instruments which is typically the backscatter strength (B-mode image). The driving hypothesis of our work is that as the ultrasound wavelength approaches the size of a cell, the backscatter characteristics become more sensitive to the cells' physical attributes (size, structure, and composition) and changes in the cell structure due to therapeutic intervention. A better understanding of the interaction of acoustic waves with cells is required to interpret the backscatter data. An overview of our efforts to model scattering from individual cells, cell ensembles, and in-vivo cancer will be presented. Theoretical results will be presented comparing scattering from individual cells with experimental data from a variety of cells in suspension, cell ensembles with pellets (compact aggregates of cells) and in-vivo tumors. It will be shown that for the tumors we have studied there is a very good correlation of scattering characteristics between cell pellets and in-vivo tumors, indicating that in the tumors studied cell structure was the factor that dominated the scattering response. The goal of this work is to use quantitative ultrasound methods for diagnosis and monitoring the response to therapy.

\section{2:40}

1pBBb6. Estimation of heterogeneity of scatterer densities for diagnosis of liver fibrosis. Tadashi Yamaguchi (Research C. for Frontier Med. Eng., Chiba University, 1-33 Yayoicho, Inageku, 263-8522 Chiba, Japan, yamaguchi@faculty.chiba-u.jp), Hiroyuki Hachiya (Dept. of Mech. and Cntr. Eng., Tokyo Inst. of Tech., 2-12-1 Ookayama, Meguroku, 152-8550 Tokyo, Japan, hachiya@ctrl .titech.ac.jp)

In the quantitative diagnosis using ultrasound, existence of the high or low echo which was indistinguishable from speckle serves as an index of diagnosis. In this study, we tried to clarify the relation between the scattering condition in the tissue and the probability density function of echo amplitude. Parameters to estimate the scattering condition were derived by Q-Q probability plot at computer simulation models and the clinical data of liver fibrosis. In the simulation model of heterogeneous medium, the result of Q-Q plot became a curve and the curvature was dependent on the difference of the scatterer density of two intermingled media. In the clinical data of liver fibrosis, curvature was large when many fibers were contained. On the other hand, curvature was small when cysts or minute blood vessels were intermingled in the speckle and the whole distribution function was able to be approximated by k-distribution. Moreover, the crooked point of the Q-Q plot was changed depending on the scatterer density of a mixture part. These results show that the mixture rate and scatterer distributions of two different distribution functions can be recognized parametrically in the liver in which minute diseased tissue was intermingled. 
1pBBb7. Noninvasive temperature estimation using diagnostic ultrasound: In vivo results. Dalong Liu (University of Minnesota, 200 Union St SE, Rm 4-174 EECS Bldg, Minneapolis, MN 55455, USA, liuxx293@umn.edu), Ajay Shrestha (University of Minnesota, 200 Union St SE, Rm 4-174 EECS Bldg, Minneapolis, MN 55455, USA, ajay@ece.umn.edu), Rachana Visaria (University of Minnesota, 200 Union St SE, Rm 4-174 EECS Bldg, Minneapolis, MN 55455, USA, visar001@umn.edu), John Bischof (University of Minnesota, 200 Union St SE, Rm 4-174 EECS Bldg, Minneapolis, MN 55455, USA, bischof@umn.edu), Emad S. Ebbini (University of Minnesota, 200 Union St SE, Rm 4-174 EECS Bldg, Minneapolis, MN 55455, USA, emad@umn.edu)

In this paper, we present temperature imaging data obtained before and after HIFU-induced lesions in implanted tumors in the hind limb of nude mice in vivo. The RF data was acquired using a $9 \mathrm{MHz} 192$-element linear probe on a diagnostic scanner at 90 frames per second. Approximately $2 \mathrm{~s}$ of frame data was collected before, during and after sub-therapeutic exposures of $0.2 \mathrm{~s}$ duration. The frame rates were sufficiently high to capture the effects of local tissue deformation due to breathing and pulsation near blood vessel. Using a physics-based spatial-temporal filtering of tissue displacement, we were able to produce accurate spatial-temporal maps of the temperature change with fine spatial and temporal resolution. These results are in good agreement with directly measured temperatures using fine temperature sensors near the HIFU focal spot. The results show that robust temperature estimation in the presence of tissue motion/deformation is feasible. Applications of this method in measuring the local thermal properties of tissue will be addressed. In particular, tissue absorption can be estimated and it appears to increase by a factor of 2 - 4 after lesion formation.

3:20

1pBBb8. Transient elastography: changing clinical practice in hepatology. Laurent Sandrin (Echosens, R\&D department, 153 avenue d'Italie, 75013 Paris, France, laurent.sandrin@echosens.com), Sylvain Yon (Echosens, R\&D department, 153 avenue d'Italie, 75013 Paris, France, sylvain.yon@echosens.com), Céline Fournier (Echosens, R\&D department, 153 avenue d'Italie, 75013 Paris, France, celine.fournier@echosens.com), Veronique Miette (Echosens, R\&D department, 153 avenue d'Italie, 75013 Paris, France, veronique.miette@echosens.com)

Transient elastography is one of several new approaches that have recently been proposed to manage liver diseases. This quantitative method is used in clinical practice to assess liver fibrosis noninvasively and rapidly. The technique consists in generating low-frequency elastic shear waves through the liver and measuring their velocity using ultrafast pulse-echo ultrasound acquisition. Liver stiffness is directly related to shear waves velocity. Nowadays more than 400 devices (Fibroscan ${ }^{\circledR}$, Echosens, Paris, France) are being used worldwide in clinical practice. Studies reported a strong correlation between liver stiffness and the fibrosis stage obtained by liver biopsy in patients with chronic liver diseases: hepatitis B and/or C, HCV co-infection, HIV, alcoholism, etc. Stiffness measurements obtained using transient elastography are in good agreement with measurements performed using other elastography techniques (magnetic resonance elastography and radiation force). Main limitations of transient elastography are related to the morphology of the patients. Examinations may be difficult or impossible in overweighted to obese patients and in children. New probes have been developed for children and obese patients. Using these probes and dedicated measurement procedures the performance of transient elastography is significantly improved. The development of transient elastography currently focuses on heterogeneous stiffness measurement.

\section{3:40-4:00 Break}

\section{Contributed Papers}

4:00

1pBBb9. Transient elastography in heterogeneous tissues. Cécile Bastard (Echosens, R\&D department, 153 avenue d'Italie, 75013 Paris, France, cecile.bastard@echosens.com), Jennifer Oudry (Echosens, R\&D department, 153 avenue d'Italie, 75013 Paris, France, jennifer.oudry@echosens.com), Yassine Mofid (Echosens, R\&D department, 153 avenue d'Italie, 75013 Paris, France, mofid@echosens.com), Laurent Sandrin (Echosens, R\&D department, 153 avenue d'Italie, 75013 Paris, France, laurent.sandrin@echosens.com)

The Fibroscan ${ }^{\circledR}$ (Echosens, Paris, France) is a transient elastography based device used to quantify liver fibrosis by following the propagation of a low frequency shear wave and measuring the mean Young's modulus of the liver. This device has been successfully applied to homogeneous tissues such as liver in patients with chronic hepatitis C. Current developments in transient elastography are now headed toward the characterization of heterogeneous tissues. The estimation of the shear wave velocity can be achieved by solving the elastic wave equation taking into account either the $1 \mathrm{D}$, the $2 \mathrm{D}$, or the $3 \mathrm{D}$ components of the displacement spatial derivatives. The objective of this study is to characterize focal nodules in human liver and to quantify heterogeneous fibrosis. We present the methods used to estimate the local shear wave velocity and the results of experiments conducted on heterogeneous phantoms and in the liver in vivo.

4:20

1pBBb10. Improvements in liver diseases evaluation using transient elastography for obese patients. Magali Sasso (Echosens, R\&D department, 153 avenue d'Italie, 75013 Paris, France, magali.sasso
@echosens.com),Veronique Miette(Echosens, R\&D department, 153 avenue d'Italie, 75013 Paris, France, veronique.miette@echosens.com), Laurent Sandrin (Echosens, R\&D department, 153 avenue d'Italie, 75013 Paris, France, laurent.sandrin@echosens.com)

Transient elastography (Fibroscan ${ }^{\circledR}$, Echosens, Paris, France) is a non invasive and rapid technique used to assess liver fibrosis by measuring liver stiffness. Clinical interest of liver stiffness measurement using Fibroscan ${ }^{\circledR}$ has been demonstrated for chronic liver diseases in adult patients. Clinically, the liver stiffness evaluation can be tricky in obese patients due to their large subcutaneous fat thickness. Obesity is a growing public health concern which requires dedicated liver evaluation procedures due to the patients' morphology and/or the specific diseases associated with obesity. Our study aims to show how Fibroscan ${ }^{\circledR}$ can adapt for liver diseases evaluation in obese patients. Clinical adaptations dedicated to over-weighted patients are shown: ultrasound guidance procedure, development of a dedicated probe for patients with a large subcutaneous fat thickness, development of new algorithms based on attenuation of both ultrasonic and shear waves. Performance is assessed and clinical interest of each adaptation is discussed in over-weighted, obese and morbid obese patients (body mass index greater than 25). These new procedures dedicated to obese patients improve liver diseases assessment using Fibroscan ${ }^{\circledR}$.

1pBBb11. Towards the modeling of high-frequency ultrasound scattering from cells. Omar Falou (Ryerson University, Department of Physics, 350 Victoria Street, Toronto, ON M5B2K3, Canada, ofalou 
@ryerson.ca),J. Carl Kumaradas(Ryerson University, Dept. of Physics, 350 Victoria Street, Toronto, ON M5B 2K3, Canada, ckumarad@ryerson.ca), Michael C. Kolios (Ryerson University, Department of Physics, 350 Victoria Street, Toronto, ON M5B2K3, Canada, mkolios@ryerson.ca)

High-frequency ultrasound is a novel method to detect cell death based on changes in cell morphology that cause alterations in the acoustic properties of cell ensembles in tissues. In previous work, our group has suggested that for cells with a nucleus to cell volume ratio of 0.33 , the backscatter response from single cells was best modeled as a fluid sphere. However, for cells with a larger nucleus, neither the fluid nor the elastic sphere models showed a good agreement. We hypothesize that cells with a large nucleus ratio $(>0.33)$ may be modeled as an elastic sphere surrounded by a fluid shell. In this work, the backscatter response from non-nucleated cells (Strongylocentrotus purpuratus oocytes) was measured Experimental RF lines were acquired using the VisualSonics VS40B ultrasound biomicroscope using 20 and $40 \mathrm{MHz}$ transducers. A finite-element model of wave propagation was developed to solve high-frequency ultrasound scattering from spheres. A very good agreement was found suggesting that the cytoplasm of the oocytes is of fluid nature. Finally, a finite-element model treating the cells as having an elastic nucleus surrounded by a fluid shell is compared to experimental measurements. The implications of these findings on the prediction of ultrasound backscatter from cells are discussed.

\section{5:00}

1pBBb12. High-frequency quantitative ultrasound imaging methods for human lymph nodes characterization ex vivo. Jonathan Mamou (Riverside Research Institute, 156 William St., 9th Floor, New York, NY 10038, USA, mamou@rrinyc.org), Alain Coron (Université Paris 6, Laboratoire d'Imagerie Paramétrique, 15, rue de l'Ecole de Médecine, 75006 Paris, France, alain.coron@lip.bhdc.jussieu.fr), Masaki Hata (J. A. Burns School of Medicine, 405 N. Kuakini Street, Honolulu, HI 96817, USA, masakihata4999@yahoo.co.jp), Junji Machi (J. A. Burns School of Medicine, 405 N. Kuakini Street, Honolulu, HI 96817, USA, junji@hawaii.edu), Eugene Yanagihara (J. A. Burns School of Medicine, 405 N. Kuakini Street, Honolulu, HI 96817, USA, kplety@earthlink.net), Pascal Laugier (Université Paris 6, Laboratoire d'Imagerie Paramétrique, 15, rue de l'Ecole de Médecine, 75006 Paris, France, laugier@lip.bhdc.jussieu.fr), Ernest J. Feleppa (Riverside Research Institute, 156 William St., 9th Floor, New York, NY 10038, USA, feleppa @ rrinyc.org)

High-frequency $(>15 \mathrm{MHz}$, i.e., wavelength $<100 \mu \mathrm{m})$ ultrasound has the potential to characterize biological tissues quantitatively at the microscopic level. In this study, quantitative ultrasound (QUS) methods were developed to evaluate freshly excised human lymph nodes from patients with possible metastatic cancer. The objectives were to improve detection of small but clinically significant lymph-node metastases that often are missed during routine histological evaluation. Three-dimensional (3D) ultrasound data acquisition was conducted on freshly excised nodes using a sphericallyfocused transducer with a center frequency of $21 \mathrm{MHz}$. Average scatterer sizes and acoustic concentrations were estimated using QUS methods with a Gaussian scattering model over a bandwidth extending from 11 to $27 \mathrm{MHz}$. Strategies were developed to recover lymph-node orientation and size after histological sectioning to allow for spatially matching QUS estimates to histology. The signal- and image-processing aspects of QUS estimation are presented and the preliminary results are shown that demonstrate our ability to acquire 3D ultrasound and histological data, to co-register them spatially, and to obtain scatterer-size and acoustic-concentration estimates for specific tissue types (cancer versus non-cancer) within the nodes. In future studies, these QUS estimates will be used to identify histologically difficult-to-detect micrometastases in lymph-nodes. [Supported by NIH R01CA100183.]

\section{$5: 20$}

1pBBb13. Extraction of the multiple scattering contribution in weakly scattering media: Application to human soft tissues. Alexandre Aubry (Laboratoire Ondes et Acoustique, ESPCI, Université Paris 7, CNRS, 10 rue Vauquelin, 75005 Paris, France, alexandre.aubry@espci.fr), Arnaud Derode (Laboratoire Ondes et Acoustique, ESPCI, Université Paris 7, CNRS, 10 rue Vauquelin, 75005 Paris, France, arnaud.derode@ujf-grenoble.fr), Mickael Tanter (Laboratoire Ondes et Acoustique, ESPCI, Université Paris 7, CNRS, 10 rue Vauquelin, 75005 Paris, France, michael.tanter@espci.fr)

Waves scattered by a weakly scattering random medium contain a predominant single scattering contribution as well as a multiple scattering contribution which is usually neglected. But its investigation can be fruitful for characterization purposes because it provides measurements of statistical parameters such as the scattering mean free path. Our aim is to extract the multiple scattering contribution in a weakly scattering random medium. The experimental set up consists in an array of programmable transducers placed in front of the sample. The impulse responses between each couple of transducers are measured and form the interelement matrix. Our technique allows the extraction of multiple scattering signals by taking advantage of their randomness, contrary to single scattering contributions which are shown to exhibit a deterministic coherence along the antidiagonals of the array response matrix, whatever the distribution of scatterers. To illustrate the interest of this technique, we applied it to a synthetic medium (Agar gel) and to breast tissues. Surprisingly, the multiple scattering contribution is far from negligible in the breast around 4.3 MHz. The temporal evolution of its intensity can provide a new tool to complete information provided by standard echography; it also constitutes an experimental test of the Born approximation.

\section{$5: 40$}

1pBBb14. Ultrasonic vibrometer for tissue characterization. James S. Martin (Georgia Institute of Technology, Mechanical Engineering, 771 Ferst Drive, Atlanta, GA 30332-0405, USA, james.martin@me.gatech.edu), Peter H. Rogers (Georgia Institute of Technology, Mechanical Engineering, 771 Ferst Drive, Atlanta, GA 30332-0405, USA, peter.rogers@me.gatech.edu), Michael D. Gray (Georgia Institute of Technology, Mechanical Engineering, 771 Ferst Drive, Atlanta, GA 303320405, USA, michael.gray@me.gatech.edu)

Ultrasonic vibrometers are well suited to a variety of tissuecharacterization tasks because they exploit the lateral resolution and depth of field available with diagnostic ultrasound systems for measurements at lower frequencies. In the past, ultrasonic vibrometers have been used to measure the motion of surfaces with high impedance contrast to their surroundings (otoliths, swim bladders, and lung tissue). New ultrasonic vibrometry techniques have been developed and tested that permit calibrated realtime (amplitude and phase) transduction of sub-nanometer-amplitude vibrations at audio frequencies. These can be configured using either analog or digital demodulation for carrier signals up to $10 \mathrm{MHz}$. In the fully digital configuration, the vibrometer offers better displacement resolution than has been previously reported for analog systems. It also has the capability to simultaneously distinguish between the displacements of multiple discrete vibrating scatterers or regions within a continuum of scatterers by exploiting a multi-sine carrier signal. Individual vibrating targets are separated by pulse compression and windowing. This reduces the problem of carrier drop-out or speckle noise that occurs when multiple reflections destructively interfere at the receiver of a narrow-band signal. The technique should permit the vibrometer a depth resolution comparable a typical pulse-echo ultrasound system. [Work supported by ONR.]

\section{6:00}

1pBBb15. An acoustic technique for mapping and sizing particles following needle-free transdermal drug and vaccine delivery. Jamie Condliffe (University of Oxford, Medical Engineering Unit, 43 Banbury 
Road, OX2 6PE Oxford, UK, jamie.condliffe@magd.ox.ac.uk),Heiko Schiffter (University of Oxford, Medical Engineering Unit, 43 Banbury Road, OX2 6PE Oxford, UK, heiko.schiffter@eng.ox.ac.uk), Constantin C. Coussios (University of Oxford, Medical Engineering Unit, 43 Banbury Road, OX2 6PE Oxford, UK, constantin.coussios@eng.ox.ac.uk)

Needle-free ballistic particle injection enables painless transdermal delivery of pharmaceuticals. To improve the efficacy of this technique, a noninvasive method of assessing particle penetration depth, distribution and changes in particle size following injection is required. Polydisperse distributions of polystyrene particles, mean diameter $25 \mu \mathrm{m}$, and insulin particles, mean diameter $44 \mu \mathrm{m}$, were injected into both tissue-mimicking phantoms and porcine skin, which they penetrate to a mean depth of $300 \mu \mathrm{m}$. Following injection, the surface of the targets was scanned using a $50 \mathrm{MHz}$ focused ultrasound transducer driven in pulse-echo mode. The received waveforms were post-processed to estimate particle penetration depth and distribution in the plane perpendicular to injection. The targets were then sectioned and optical microscopy was used to validate the acoustic results. Furthermore, computational implementation of an exact solution for sound scattering by an elastic sphere allows prediction of the frequency response of the insonified particles. Direct comparison of the theoretical model with the frequency content of experimental data is shown to provide an accurate means of estimating particle size, and is being extended to correlating particle size with penetration depth. The acoustic technique shows great promise as a noninvasive means of mapping, sizing, and assessing stability of drug particles following needle-free injection.

\section{6:20}

1pBBb16. Noninvasive vascular ultrasound elastography of carotid arteries for stroke prevention. Guy Cloutier (Laboratory of Biorheology and Medical Ultrasonics, University of Montreal Hospital Research Center, 2099 Alexandre de Sève (room Y-1619), Montreal, QC H2L 2W5, Canada, guy.cloutier@umontreal.ca), Roch Maurice (Laboratory of Biorheology and Medical Ultrasonics, University of Montreal Hospital Research Center, 2099 Alexandre de Sève (room Y-1619), Montreal, QC H2L 2W5, Canada, maurice.roch.chum@ssss.gouv.qc.ca), Elizabeth Mercure (Laboratory of Biorheology and Medical Ultrasonics, University of Montreal Hospital Research Center, 2099 Alexandre de Sève (room Y-1619), Montreal, QC H2L 2W5, Canada, elizabeth.mercure@UMontreal.CA), Marie-France Giroux (Department of Radiology, University of Montreal Hospital, 1560 Sherbrooke Est, Montreal, QC H2L 4M1, Canada, m_fgiroux@videotron.ca), Gilles Soulez (Department of Radiology, University of Montreal Hospital, 1560 Sherbrooke Est, Montreal, QC H2L 4M1, Canada, gilles.soulez.chum@ssss.gouv.qc.ca)

Carotid plaque rupture leading to stroke involves alteration of arterial wall mechanical properties. This paper addresses reproducibility and potential clinical impact of non-invasive vascular elastography. Fifteen symptomatic and 15 asymptomatic patients with greater than 50\% stenoses of their internal carotids, and 15 control subjects were scanned independently by two radiologists. Radio-frequency cine-loops were acquired on left and right common and proximal internal carotids. For control subjects, homogeneous strains with successive compression and dilatation of the vascular wall from systole to diastole and few shear patterns were observed. The axial strain did not differ between recording sites, sides and radiologists (ANOVA, $\mathrm{p}>$ 0.19). For patients, heterogeneous axial strain patterns with both compression and dilatation of tissues within plaques were noted along with shear concentrations at interfaces of tissue structures. Despite similar blood pressures, larger mean instantaneous axial strains were measured in symptomatic $(1.5 \pm 0.6 \%)$ versus asymptomatic $(1.2 \pm 0.5 \%)$ patients in systole for com- mon carotids (t-test, $\mathrm{p}<0.02$ ). To conclude, reproducible results were obtained for control subjects and in patients, larger axial strains in common carotids may be associated with plaque rupture. This new imaging method may become a unique approach to characterize vulnerable plaques for stroke prevention.

1pBBb17. ANAIS: An ultrasound mammograph. Serge Mensah (Laboratory for Mechanics and Acoustics CNRS, 31 chemin Joseph Aiguier, 13009 Marseille, France, mensah@lma.cnrs-mrs.fr), Philippe Lasaygues (Laboratory for Mechanics and Acoustics CNRS, 31 chemin Joseph Aiguier, 13009 Marseille, France, lasaygues@lma.cnrs-mrs.fr), Eric Debieu (Laboratory for Mechanics and Acoustics CNRS, 31 chemin Joseph Aiguier, 13009 Marseille, France, debieu@1ma.cnrs-mrs.fr), Emilie Franceschini (CNRS - LMA, 31 Chemin Joseph Aiguier, 13009 Marseille, France, franceschini@lma.cnrs-mrs.fr)

It is well established that ultrasonography is a cost-effective, patientfriendly imaging modality available for breast imaging. The main aim of this paper is concerned with a tomographic ultrasound mammograph allowing 3D-reconstruction of the uncompressed breast immersed in a water tank. The scanner, based on a 1024 semi-circular motorized antenna, provides more efficient way to view anatomical ductal structures of the breast. Quantitative volume parameter cartography is the unique means to make objective, reproducible, and operator-independent diagnoses (Computer Aided Diagnosis). The recovery of quantitative acoustical parameters requires solving a nonlinear inverse scattering problem from large amount of measurements. This is achieved using advanced computing resources; a front-end acquisition system based on $32 * 1024$ multiplexed channels and specific reconstruction procedures. The objective is not only to offer a multiparameter tissue characterization using reflection and transmission measurements but also to provide a systemic inspection of the ductal tree.

\section{7:00}

1pBBb18. A reflex transmission method for ultrasound thermometry. Caleb H. Farny (Harvard Medical School, Focused Ultrasound Lab - BWH Radiology, 221 Longwood Ave RM 521, Boston, MA 02115, USA, cfarny@bwh.harvard.edu), Gregory Clement (Harvard Medical School, Focused Ultrasound Lab - BWH Radiology, 221 Longwood Ave RM 521, Boston, MA 02115, USA, gclement@hms.harvard.edu)

It is of great interest to develop ultrasound-based thermal imaging for monitoring thermal ablation procedures and as a tissue characterization technique. A phase contrast analysis for temperature mapping has been investigated, based on the temperature dependence of sound speed of water and tissue. The method employs reflex transmission imaging principles, planar projection and tomographic reconstruction techniques, for interrogating a region featuring a temperature contrast. Through-transmission and pulseecho configurations were both used to image phase contrasts caused from a change in sound speed, in experimental measurements and numerical simulations. Tissue phantoms were used as a medium in which to create a steady-state sound speed contrast, and a raster scan was performed to acquire the sound field with a hydrophone. Two scans were acquired, a reference sound field and a scan featuring a phase contrast which corresponded to a $7-10^{\circ} \mathrm{C}$ peak temperature rise. The through-transmission configuration employed a $0.2-\mathrm{mm}$ diameter needle hydrophone, while the pulse-echo measurement employed a custom-built thin-wire hydrophone to acquire the sound field between the interrogation transducer and tissue phantom. The method successfully located the location and amplitude of the phase contrast, and future steps necessary for tracking dynamic temperature changes will be discussed. 


\title{
Session 1pBBc
}

\section{Biomedical Ultrasound/Bioresponse to Vibration and Engineering Acoustics: High-Intensity Focused Ultrasound Metrology and Standards I}

\author{
Peter Kaczkowski, Cochair \\ Center for Industrial and Medical Ultrasound, Applied Physics Lab., University of Washington, 1013 NE 40th St., \\ Seattle, WA 98105, USA \\ Gail Ter Haar, Cochair \\ Institute of Cancer Research, 15 Cotswold Road, Belmont, Sutton, Surrey, SM2 5NG, UK
}

\section{Invited Papers}

\author{
$5: 40$
}

1pBBc1. On the need for basic theoretical, numerical, and experimental research in the development of useful standards for focused ultrasound therapy. Peter Kaczkowski (Center for Industrial and Medical Ultrasound, Applied Physics Lab., University of Washington, 1013 NE 40th St., Seattle, WA 98105, USA, peter@apl.washington.edu), Michael R. Bailey (Center for Industrial and Medical Ultrasound, Applied Physics Lab., University of Washington, 1013 NE 40th St., Seattle, WA 98105, USA, bailey@apl.washington.edu), Vera A. Khokhlova (Center for Industrial and Medical Ultrasound, Applied Physics Lab., University of Washington, 1013 NE 40th St., Seattle, WA 98105, USA, vera@acs366.phys.msu.ru), Oleg A. Sapozhnikov (Center for Industrial and Medical Ultrasound, Applied Physics Lab., University of Washington, 1013 NE 40th St., Seattle, WA 98105, USA, oleg@acs366.phys .msu.ru)

High intensity focused ultrasound (HIFU) is rapidly gaining widespread clinical use in China, and is undergoing regulatory evaluation in Europe and the US for many target diseases. There is rising concern within the HIFU community that no standards exist for measuring or reporting of HIFU fields, inhibiting broad clinical adoption of HIFU. This paper addresses current technical gaps for the development of such standards. High power and strong focusing combine to make accurate measurements of focal fields difficult. No simple way exists to extrapolate in-water field measurements directly to patients, and thus to calculate temperature rise and therapeutic dose in tissue. To arrive at a set of characteristic parameters for HIFU systems that are both clinically relevant and are practical to measure reliably, we believe several avenues of research are needed. Numerical models that include nonlinear acoustic propagation must be carefully validated with experiments, and both numerics and experimental methods must be standardized for broad dissemination. Mechanisms of enhanced tissue heating due to nonlinear effects must be experimentally quantified, and robust accurate models developed. These advances in understanding must be transitioned into practical recommendations for standard metrics and methodologies to facilitate industrial development and regulatory oversight.

\section{6:00}

1pBBc2. A method of HIFU field characterization in water and deration to tissue. Vera A. Khokhlova (Center for Industrial and Medical Ultrasound, Applied Physics Lab., University of Washington, 1013 NE 40th St., Seattle, WA 98105, USA, vera@acs366.phys.msu.ru), Michael S. Canney (Center for Industrial and Medical Ultrasound, Applied Physics Lab., University of Washington, 1013 NE 40th St., Seattle, WA 98105, USA, mcanney@u.washington.edu), Michael R. Bailey (Center for Industrial and Medical Ultrasound, Applied Physics Lab., University of Washington, 1013 NE 40th St., Seattle, WA 98105, USA, bailey@apl.washington.edu), Olga V. Bessonova (Center for Industrial and Medical Ultrasound, Applied Physics Lab., University of Washington, 1013 NE 40th St., Seattle, WA 98105, USA, olga@acs366.phys.msu.ru), Oleg A. Sapozhnikov (Center for Industrial and Medical Ultrasound, Applied Physics Lab., University of Washington, 1013 NE 40th St., Seattle, WA 98105, USA, oleg@acs366.phys.msu.ru), Lawrence A. Crum (Center for Industrial and Medical Ultrasound, Applied Physics Lab., University of Washington, 1013 NE 40th St., Seattle, WA 98105, USA, lac@apl.washington.edu)

Acoustic characterization of nonlinear HIFU fields is important for both the accurate prediction of ultrasound induced bioeffects and the development of regulatory standards for clinical HIFU devices. In this work a new characterization method is proposed and tested in water, tissue phantoms, and ex-vivo tissues. The method is based on the combined use of measurements and modeling. Experiments were performed with a $2 \mathrm{MHz}$ transducer of $4.2 \mathrm{~cm}$ aperture and $4.5 \mathrm{~cm}$ focal length. Low amplitude measurements in water were used to establish boundary conditions for modeling based on the KZK-type equation. High amplitude focal waveforms then were simulated and measured with a fiber optic probe hydrophone in water, within tissue phantom, or adjacent to excised tissue. It was shown that at high amplitudes, the simulations of shock waveforms were more accurate than the measurements. The focal waveforms obtained in water were found to be in a good agreement with those produced in tissue with higher source pressure scaled to compensate for the linear attenuation on the way to focus. This result establishes a method to derate the focal HIFU pressures determined in water to tissue. [Work supported by NIH DK43881, NSBRI SMS00402 and RFBR.] 
1pBBc3. Uncertainty of power and intensity measurement in focused fields. Adam Shaw (National Physical Laboratory, Hampton Road, Teddington, TW11 0LW Middlesex, UK, adam.shaw@npl.co.uk), Pierre Gelat (National Physical Laboratory, Hampton Road, Teddington, TW11 0LW Middlesex, UK, pierre.gelat@npl.co.uk)

Currently, the methodology employed to measure acoustic intensity and acoustic power relies on the plane wave assumption, resulting in a simple relation between acoustic power and the normal component of the radiation force vector, and acoustic intensity and pressure magnitude. For a range of ultrasonic transducers, and for positions in the field beyond the last axial maximum, this assumption is a valid one. Nevertheless, there is a range of situations where the plane wave assumption may introduce significant uncertainty in the measurement, such as in near field measurements and in fields resulting from highly focused devices. This investigation looks at the acoustic fields generated by axisymmetric rigidly vibrating curved pistons over a range of diameters and radii of curvature at megahertz frequencies. Finite element and boundary element methods are used to evaluate the acoustic pressure field and the components of the particle velocity vector at specified field positions. The time-averaged acoustic intensity vector, the acoustic power, as well as the normal component of the radiation force vector on a perfectly absorbing target may then be derived. Experimental results are will also be presented comparing theory with radiation force measurements and with the new and more accurate buoyancy change method.

\section{6:40}

1pBBc4. Towards a standardized approach for quantifying inertial cavitation activity. Jamie R. Collin (University of Oxford, Medical Engineering Unit, 43 Banbury Road, OX2 6PE Oxford, UK, jamie.collin@magd.ox.ac.uk), Ian Webb (University of Oxford, Medical Engineering Unit, 43 Banbury Road, OX2 6PE Oxford, UK, ian.webb@eng.ox.ac.uk), Ronald A. Roy (Boston University, Dept. of Aerosp. and Mech. Eng., 110 Cummington St., Boston, MA 02215, USA, ronroy@bu.edu), Constantin C. Coussios (University of Oxford, Medical Engineering Unit, 43 Banbury Road, OX2 6PE Oxford, UK, constantin.coussios@eng.ox.ac.uk)

Relative measures of the energy radiated as broadband noise emissions by inertially cavitating bubbles, such as the mean square voltage received using a passive cavitation detector (PCD), have been recently shown to be directly relatable to cavitation-mediated bioeffects. Even though numerous techniques exist to detect inertial cavitation qualitatively, there is presently a lack of a unified approach that makes it possible to quantify and compare levels of cavitation activity across different experimental setups and conditions. A technique has been developed that uses a calibrated sound source, a hydrophone, and a point spherical scatterer of known frequency response embedded in a tissue-mimicking material to achieve an absolute calibration of a PCD on receive. This makes it possible to relate the PCD signal during inertial cavitation activity to the acoustic power radiated as broadband noise in the proximity of the bubble cloud. The technique is validated against PCD measurements of single-bubble inertial cavitation activity in a tissue-mimicking material and compared to theoretical predictions using the Keller-Miksis model. Calibrated measurements of acoustic power radiated as broadband noise emissions could thus provide a quantitative measure of cavitation dose that can be directly related to resulting bioeffects, such as increased sonoporation or enhanced heating.

\section{7:00}

1pBBc5. Standardisation and metrology for High Intensity focused ultrasound: a clinical perspective. Gail Ter Haar (Institute of Cancer Research, 15 Cotswold Road, Belmont, Sutton, SM2 5NG Surrey, UK, gail.terhaar@icr.ac.uk)

The clinical application of HIFU is a young and rapidly expanding field, and it is essential that validated methods for measurement and testing are made available as soon as possible. These issues have not been addressed to date in any systematic fashion. There is, therefore, an urgent need to produce standard registration, testing equipment and methodology to allow users to characterize clinical HIFU systems for checking safety and reproducibility of a machine's output, comparing different devices or commissioning new systems. HIFU is delivered to the target volume via a number of routes. Extra-corporeal and trans-rectal probes are the most commonly used, although preliminary testing of intra-cavitary, catheter based devices is also underway 19. These different devices present a wide range of different transducer geometries, f-numbers $(\sim 0.8-1.8)$, operating frequencies $(\sim 0.8-5 \mathrm{MHz})$, and focal peak intensities $(\sim 7.5$ $10^{2}-3104 \mathrm{~W} \mathrm{~cm}^{-2}$ ). Clinical HIFU systems are currently assessed on an ad hoc basis by individual clinical departments and manufacturers, using methods, many of which are unpublished. The main requirements from a clinical perspective are for the quantification of parameters which relate directly to individual HIFU treatments, allow reproducible treatments between different patients, and allow clinical trial data to be compared. 


\title{
Session 1pEAa
}

\section{Engineering Acoustics, Underwater Acoustics, Signal Processing in Acoustics, and ECUA: Sensor Technologies for Autonomous Acoustic Sensing Systems I}

\author{
Henrik Schmidt, Cochair \\ MIT, 77 Massachusetts Ave, 5-204, Cambridge, MA 02139, USA \\ Andrea Caiti, Cochair \\ University of Genova, Via Opera Pia 13, 15614 Genova, Italy
}

\section{Contributed Paper}

\section{1:00}

1pEAa1. SLITA: A new slim towed array for AUV applications. Alain Maguer (NURC Nato Undersea Research Centre, Viale San Bartolomeo 400, 19026 La Spezia, Italy, maguer@nurc.nato.int), Rodney Dymond (NURC Nato Undersea Research Centre, Viale San Bartolomeo 400, 19026 La Spezia, Italy, dymond@nurc.nato.int), Marco Mazzi (NURC Nato Undersea Research Centre, Viale San Bartolomeo 400, 19026 La Spezia, Italy, mazzi@nurc.nato.int), Stefano Biagini (NURC Nato Undersea Research Centre, Viale San Bartolomeo 400, 19026 La Spezia, Italy, biagini@nurc.nato.int), Stefano Fioravanti (NURC Nato Undersea Research Centre, Viale San Bartolomeo 400, 19026 La Spezia, Italy, fioravanti@ nurc.nato.int), Piero Guerrini (NURC Nato Undersea Research Centre, Viale San Bartolomeo 400, 19026 La Spezia, Italy, guerrini@nurc nato.int)

The typical $70 \mathrm{~mm}$ diameter towed array was developed for blue-water detection at long range and low frequencies in the 1960s. Since then, there has been a need for towed arrays that are lighter and less expensive, espe- cially since the maturing field of autonomous vehicles has expanded the potential of such arrays. The marriage of AUVs and lightweight towed arrays is a natural progression in the development of littoral autonomous sensing networks for applications such Anti-Submarine Warfare, marine mammals, or ambient noise measurements. In August 2007, NURC began to design and build a new thin diameter (31 mm) high-frequency (up to $20 \mathrm{kHz}$ ) nested towed array for ASW purposes. An engineering at-sea trial of the array towed by OEX AUV was performed beginning of November 2007. The flow noise level of the array while towed and the potential influence of the AUV self-noise on the acoustic array were also measured. This paper will first describe the array design, its acquisition system and its integration on the OEX AUV. Then, the results obtained from the data analysis are presented. It is shown that the SLITA array has performance that will make it easily fit requirements of the applications previously mentioned.

\section{Invited Paper}

$1: 20$

1pEAa2. An autonomous moored ocean profiler. Benjamin Cray (Naval Undersea Warfare Center, Code 821, 1176 Howell Street, Newport, RI 02841-1708, USA, crayba@npt.nuwc.navy.mil)

An unconventional set of environmental and acoustic data, measured from an autonomous moored ocean profiler, is presented. The profiler is a programmable robotic underwater winch and data acquisition system that cycles vertically through the water column. The system profiles at pre-determined time intervals, surfaces to transmit data via RF links, and can be deployed for up to 180 days. Built by WETLabs Incorporated (Western Environmental Technology Laboratories) for the Naval Underwater Systems Center (NUWC), the system is outfitted with three environmental sensors and two acoustic vector sensors. The environmental sensors include; a Nortek Vector velocimeter which samples current components and characterizes surface wave velocities, a Seabird FastCAT Conductivity, Temperature, Depth (CTD) sensor; and a Wetlabs Fluorometer-Turbidity optical sensor that measures both chlorophyll-a and turbidity. The Wilcoxon acoustic vector sensors measure acoustic pressure and the three components of acoustic particle acceleration. Wilcoxon Research (a subsidiary of Meggitt Corporation) and Applied Physical Sciences (APS) have developed underwater acoustic vector sensors for many applications. The quality and sensitivity of these vector sensors has steadily improved. A review of early (pre-2002) vector sensor development efforts is discussed.

\section{Contributed Papers}

1:40

1pEAa3. Experimental validation of a chirp-based underwater acoustic communication method. Maria Palmese (DIBE - Dept. of Biophysical and Electronic Engineering - University of Genoa, Via Opera Pia 11a, 16145 Genova, Italy, palmese@dibe.unige.it), Giacomo Bertolotto (Fincantiere Cantieri Navali Italiani S.p.A., Via Cipro 11, 16126 Genova, Italy, Giacomo.bertolotto@fincantieri.it), Alessandro Pescetto (Vulnerability \& Signature Department, CETENA S.p.A., Via Ippolito d'Aste 5, 16121 Genova, Italy, alessandro.pescetto@cetena.it), Andrea Trucco (DIBE Dept. of Biophysical and Electronic Engineering - University of Genoa, Via Opera Pia 11a, 16145 Genova, Italy, trucco@ieee.org)
A wireless underwater acoustic communication algorithm based on the combination of chirp modulation and direct-sequence spread-spectrum signaling is presented. In this paper, the processing chain design is proposed, discussed, and demonstrated using real data. The communication algorithm is made of a transmission block encoding the bits with adequate linear chirps multiplied by pseudo noise (PN) sequences, and a rake receiver that allows one to positively exploit the energy present in the most significant propagation paths. The use of chirp signals takes advantage of the low Doppler sensitivity in the matched filter operation whereas the choice of PN sequences allows one to reduce narrowband interference arising from other users and self-interference due to multipath propagation. Moreover a tracking 
procedure that allows an adaptation to the instantaneous Doppler shift has been devised and tested. Some experiments have been carried out changing the distance and the speed between transmitter and receiver. Results show that the developed communication method is able to handle the multipath phenomenon and the Doppler effect, allowing one to achieve a bit error rate less than $10(-3)$ for long ranges for bit rates of about 15 and $230 \mathrm{bit} / \mathrm{s}$.

\section{2:00}

1pEAa4. Broadband transducers for underwater communications. David Brown (BTech Acoustics, LLC and Universtity of Massachusetts Dartmouth, ATMC, Electro-Acoustics Research Laboratory and ECE Dept., 151 Martine St, Fall River, MA 02723, USA, dbAcoustics@ cox.net), Corey Bachand (BTech Acoustics, LLC and Universtity of Massachusetts Dartmouth, ATMC, Electro-Acoustics Research Laboratory and ECE Dept., 151
Martine St, Fall River, MA 02723, USA, corey.bachand@cox.net),Boris Aronov (BTech Acoustics, LLC and Universtity of Massachusetts Dartmouth, ATMC, Electro-Acoustics Research Laboratory and ECE Dept., 151 Martine St, Fall River, MA 02723, USA, baronov@comcast.net)

Properties of underwater acoustic cylindrical piezoelectric (PZT) transducers currently used for broadband underwater communications with unmanned underwater vehicles (UUV) and new transducers under development utilizing single crystal relaxor ferroelectrics (PMN-PT) with significantly higher material coupling coefficients and lower sound speed are presented. Characteristics including resonance frequency, coupling coefficient, transmit (TVR) and receive (OCVS) frequency response, maximum drive and achievable source levels, tuned and untuned power factor, and directivity factors are discussed. Results for experimental calibrations and field tests will be compared with detailed equivalent electrical circuit models of the transducer and transducer channel. Work supported in part by BTech Acoustics and Office of Naval Research.

\section{Invited Papers}

1pEAa5. High resolution angular measurements with single vector sensors and arrays. Joseph A. Clark (NSWCCD, Code 7340, 9500 MacArthur Blvd., West Bethesda, MD 20817-5000, USA, joseph.a.clark1@ navy.mil), Dehua Huang (Naval Undersea Warfare Center, 1176 Howell St., Newport, RI 02841, USA, HuangD@ Npt.NUWC.Navy.Mil)

The direction finding capability of acoustic sensors that directly measure acoustic particle velocity alone or in combination with pressure measurements (vector sensors) is well known. The angular resolution of the measurements can be improved by using arrays of vector sensors. It is also possible to improve angular resolution by additional processing of data from even a single vector sensor. This approach could be especially advantageous for sonar systems designed to operate from small platforms such as autonomous underwater vehicles. In this talk linear and non-linear methods for processing data from one or more vector sensors to achieve high angular resolution will be reviewed. Experimental investigations comparing the methods will be reported and factors found to limit the localization and detection performance of sonar systems employing the methods will be discussed.

\section{2:40}

1pEAa6. Hydroflown: MEMS-based Underwater Acoustical Particle Velocity Sensor. Tuncay Akal (SUASIS: Underwater Systems Technology Development, Tubitak-Marmara Research Center, Tech. D. Free Zone, Block A - L4, Gebze, 41470 Kocaeli, Turkey, tuakal@yahoo.com), Hans-Elias De Bree (Microflown Technologies Inc., PO BOX 300, 6900 AH Zevenaar, Netherlands, debree@microflown.com), Piero Guerrini (NURC Nato Undersea Research Centre, Viale San Bartolomeo 400, 19026 La Spezia, Italy, guerrini@nurc.nato.int), Alain Maguer (NURC Nato Undersea Research Centre, Viale San Bartolomeo 400, 19026 La Spezia, Italy, maguer@nurc.nato.int)

The increasing problems related to homeland security and harbour/infrastructure protection have increased the level of interest on vector sensors. Market surveys carried out during the last three years gave the conclusion that there is a need for a new generation, small size, and low-cost underwater sensors capable of measuring particle velocity in three dimensional plain within a broad frequency band $(2 \mathrm{~Hz}-50 \mathrm{kHz})$ and with high angular resolution. The small size MEMS-based sensors developed by Microflown Technologies BV Inc are the world's only commercially available transducers that are capable of measuring the particle velocity, instead of pressure, in air. The development of a new generation, innovative and low-cost underwater sensors and technologies based on that in-air nano technology is therefore considered. This technology has a great potential to become a revolutionary underwater acoustic sensor using nanotechnology, capable of finding many applications like sensors for Autonomous Underwater Vehicles, sensors for directional receivers for underwater acoustic systems, Floating autonomous systems, Sensors for seismic towed arrays for underwater oil and mineral prospecting and harbour and water-side infrastructure protection. This paper describes how Microflown technology can be adapted to underwater applications.

\section{Contributed Papers}

\section{3:00}

1pEAa7. Concurrent detection, classification and localization of seabed targets using virtual time reversal. Alexis J. Dumortier (MIT, 77 Mass Ave, 5-204, Cambridge, MA 02139, USA, adumorti@mit.edu), Henrik Schmidt (MIT, 77 Mass Ave, 5-204, Cambridge, MA 02139, USA, henrik@mit.edu), Karim G. Sabra (Georgia Institute of Technology, School of Mechanical Engineering, 771 Ferst Drive, NW, Atlanta, GA 30332-0405, USA, karim.sabra@me.gatech.edu)

During the last decade, the use of unmanned vehicles to detect seabed objects has been revolutionized by the rapid development of AUV technology. The reduced power budget imposed by autonomous operations limits the amount of processing allowable onboard and calls for simple and efficient detection algorithms. Time reversal techniques have proved to be a robust way of focusing sound on reflective objects in complex environments and only require minimal computation. The method proposed here uses a virtual time reversal mirror in monostatic configuration to localize the origin of the field scattered by objects located on the seafloor. The waveguide is insonified with a low frequency source $(\sim \mathrm{kHz})$ mounted on the AUV and the resulting scattered field is sampled by a receiving array towed behind the vehicle. The recorded signals are then used to simulate the time reversed transmissions onboard, generating a map of reflectors present on the seabed. The rejection of clutter using singular value decomposition and the sensitivity of this method to environmental misknowledge are addressed along with the presentation of experimental results. [Work supported by ONR.] 


\section{3:40}

1pEAa8. Computational fluid dynamic analysis of optimized windscreens for UAV based acoustic arrays. Wayne E. Prather (Miltec Research \& Technology, 9 Industrial Park Dr., Oxford, MS 38655, USA, wprather@mil-tec.com), David H. Bridges (Mississippi State University, Dept. of Aerospace Engineering, Mississippi State, MS 39762, USA, dbridges@AE.MsState.EDU), Tom Edwards (Raspet Flight Research Laboratory, 114 Airport Road, Starkville, MS 39759, USA, edwards@raspet.msstate.edu), David S. Thompson (Mississippi State University, Dept. of Aerospace Engineering, Mississippi State, MS 39762, USA, dst@simcenter.msstate.edu)

As part of an overall effort to place acoustic sensors on airborne platforms for surveillance applications, analysis studies were performed of acoustic probe windscreens that could minimize wind noise and be made relatively insensitive to small angle of attack and sideslip excursions. Through a collaborative effort between Miltec Research \& Technology, Mississippi State University Department of Aerospace Engineering, Raspet Flight Research Laboratory, and the Engineering Research Center several probe designs were developed and analyzed using inviscid and viscous simulations with the objective of characterizing their flow fields and identifying potential modifications that could improve suitability for use as acoustic measurement probes. Several of the recommended probe shapes were fabricated and tested under real world conditions on an acoustically optimized 1/3 scale Schleicher ASH26 glider test bed.
1pEAa9. Acoustical methods for azimuth, range and heading estimation in underwater swarms. Uwe R. Zimmer (The Australian National University, $0200 \quad$ Canberra, Australia, uwe.zimmer@ieee.org), Navinda Kottege (The Australian National University, 0200 Canberra, Australia, navinda.kottege@anu.edu.au)

Acoustical Methods for Azimuth, Range and Heading Estimation in Underwater Swarms Enhanced, embodied autonomy in small submersibles enables the design and deployment of practical swarms of autonomous underwater vehicles (AUVs). The swarming paradigm requires for each vehicle location awareness of at least its near neighbors. The Serafina AUV swarming project additionally requires a localisation system which could cope with the dynamic and fast changing vehicle configurations while being small, reliable, robust, and energy efficient and not dependent on pre-deployed acoustic beacons. The short-range acoustical relative localisation system proposed here, uses hyperbolic and spherical localization concepts and provides each vehicles with the azimuth, range and heading of its near neighbours. The implementation utilises an acoustically transmitted MLS signal which provides extremely high robustness against interference by stochastic and systematic disturbances which are typical for underwater environments. The azimuth is obtained via hyperbolic positioning with improved resolution and accuracy with respect to conventional methods. Range and heading estimation is achieved by two independent methods for increased robustness; one uses the implicit synchronisation provided by the underlying inter-vehicle communication scheduling system to measure the difference of TOAs of an acoustic and a long-wave radio signals; the second relies on TDOAs and a reverse hyperbolic localisation scheme.

\section{Invited Paper}

1pEAa10. Integrated sensing, modeling, and control in undersea sensor networks. Arjuna Balasuriya (MIT, 77 Mass Ave, 5-204, Cambridge, MA 02139, USA, arjunab@mit.edu), Henrik Schmidt (MIT, 77 Mass Ave, 5-204, Cambridge, MA 02139, USA, henrik@mit.edu), Michael B. Benjamin (MIT, 77 Mass Ave, 5-204, Cambridge, MA 02139, USA, mikerb@csail.mit.edu)

The way forward in adaptively sampling the vast ocean environment is by deploying a network of sensors with different capabilities. Due to the complex nature of the environment, covering such an area cannot be achieved by simply deploying underwater sensor nodes at predefined locations. The undersea network topology should dynamically adapt to the events and changes in the environment. However, the main challenge for undersea network autonomy is due to the limitations in underwater acoustic communication. In order to overcome such limitations, Nested Autonomy architecture is being developed and demonstrated in programs such as PLUSNet and Ocean Observatories. This paper looks into the implementation of such autonomy architecture in different sensor nodes with different capabilities. This is achieved by separating the "low-level" tasks of a sensor node with its "high-level" tasks. Low-level tasks will be sensor specific while the high-level tasks looks into the autonomy required for the adaptive sampling applications, which here refers to as the "Backseat-driver paradigm". The "backseat" carries the on-board intelligence required for the mission at hand. Behavior-based autonomy architecture which is used in the back-seat significantly improves the sensor performance by selecting the best behavior for the given state of the vehicle, mission at hand and the observations being made.

\section{Contributed Paper}

\section{4:40}

1pEAa11. Artificial characteristic landmarks for SLAM navigation. Yan Pailhas (Ocean Systems Laboratory, School of Eng. \& Phys. Sciences, Heriot-Watt University, EH14 4AS Edinburgh, UK, Y.Pailhas@hw.ac.uk), Chris Capus (Ocean Systems Laboratory, School of Eng. \& Phys. Sciences, Heriot-Watt University, EH14 4AS Edinburgh, UK, C.Capus@hw.ac.uk), Keith E. Brown (Ocean Systems Laboratory, School of Eng. \& Phys. Sciences, Heriot-Watt University, EH14 4AS Edinburgh, UK, K.E.Brown@hw.ac.uk)

The use of autonomous underwater vehicles (AUVs) for a variety of purposes is set to increase in the future. A key issue in the navigation processing, especially for survey applications, is the lack of accuracy or cumulative error introduced by the various position sensors: accelerometer; DVL; compass. Algorithms such as SLAM (simultaneous localisation and mapping) rely on accurate landmark recognition in order to correct the vehicle position. This paper proposes a solution based on broadband sonar and artificial coded landmarks to improve the navigation. Through resolution of the wave equation for acoustic propagation in a multilayer concentric sphere, we will show that there is a great diversity in the echo spectrum with small changes in internal structure. This enables the design of a set of passive landmarks which can be identified unambiguously, since each has a characteristic signature or 'spectral code' when insonified with a broadband sonar.

\section{Invited Paper}

1pEAa12. Acoustic sensor systems on a flying wing underwater glider and two prop-driven autonomous underwater vehicles. Gerald D’Spain (Marine Physical Lab, Scripps Institution of Oceanography, 291 Rosecrans St., San Diego, CA 92106, USA, gld@mpl.ucsd.edu), Richard Zimmerman (Marine Physical Lab, Scripps Institution of Oceanography, 291 Rosecrans St., San Diego, 
CA 92106, USA, richard@mpl.ucsd.edu),Scott A. Jenkins (Marine Physical Lab, Scripps Institution of Oceanography, 291 Rosecrans St., San Diego, CA 92106, USA, sjenkins@ucsd.edu), Dennis B. Rimington (Marine Physical Lab, Scripps Institution of Oceanography, 291 Rosecrans St., San Diego, CA 92106, USA, drimington@ucsd.edu), James C. Luby (Applied Physics Lab, Univ. of Washington, 1013 NE 40th St., Seattle, WA 98105, USA, jcl@apl.washington.edu), Peter Brodsky (Applied Physics Lab, Univ. of Washington, 1013 NE 40th St., Seattle, WA 98105, USA, brodsky@apl.washington.edu)

The Marine Physical Laboratory, Scripps Institution of Oceanography operates several underwater vehicles including an autonomous underwater glider based on a flying wing design and two prop-driven autonomous underwater vehicles (AUV) manufactured by Bluefin Robotics. The objective of this presentation is to describe the acoustic sensor systems on these platforms and provide sample results from the at-sea data. The glider, with a 6.1-m wing span, was developed jointly by the Marine Physical Lab and the Applied Physics Laboratory, University of Washington. It is designed to maximize the horizontal distance traveled between changes in buoyancy (i.e., maximize its "finesse") while quietly listening to sounds in the ocean. A 27 -element hydrophone array with $5 \mathrm{kHz}$ per channel bandwidth is located in the sonar dome all along the wing's leading edge. In addition, it has a four-component acoustic vector sensor in its nose. The two prop-driven AUVs have been equipped with hull-mounted hydrophone arrays with $10 \mathrm{kHz}$ bandwidth for passive synthetic aperture studies, an acoustic vector sensor, and active acoustic imaging systems for ocean bottom/subbottom mapping. Results from the data processing illustrate the tight coupling between acoustic sensor systems, signal/array processing methods, and vehicle behavior. [Work supported by the Office of Naval Research.]

MONDAY AFTERNOON, 30 JUNE 2008

ROOM 353, 1:00 TO 4:00 P.M.

\title{
Session 1pEAb
}

\section{Engineering Acoustics and Signal Processing in Acoustics: Microphone Array Signal Processing I}

\author{
Gary Elko, Cochair \\ mh acoustics LLC, 25-A Summit Ave., Summit, NJ 07901, USA \\ Walter Kellermann, Cochair \\ Univ. of Erlangen-Nuremberg, 90158 Erlangen, Germany
}

\section{Contributed Papers}

\section{1:00}

1pEAb1. Spherical harmonic analysis applied to the reconstruction of the sound field radiated by a loudspeaker. Filippo M. Fazi (ISVR, University of Southampton, Highfield, SO171BJ Southampton, UK, ff1@isvr.soton.ac.uk), Vincent Brunel (ISVR, University of Southampton, Highfield, SO171BJ Southampton, UK, Vincent.Brunel@eurocopter.com), Philip A. Nelson (ISVR, University of Southampton, Highfield, SO171BJ Southampton, UK, P.A.Nelson@soton.ac .uk)

The three dimensional acoustic radiation patterns generated by different models of loudspeaker have been measured in an anechoic environment using an array of 40 omnidirectional microphones, arranged on a hemispherical surface. The acquired data have been numerically processed in order to obtain the complete three dimensional reconstruction of the analysed sound field. The theory of the applied method is based on the spherical harmonic decomposition of the sound field defined over the surface of a sphere containing the sound source and sampled at a finite number of positions. The reconstruction of the sound field in the region of the space inside and outside the measurement surface has been obtained by multiplying each coefficient of the spherical harmonic series by the corresponding spherical propagator, defined as the ratio of two spherical Hankel functions. The conditioning of the inverse problem related to the reconstruction of the sound field in the interior of the measurement surface has been analysed and put in relation to the behaviour of the spherical propagators of different orders.

\section{1:20}

1pEAb2. Surround sound echo cancellation in the spherical harmonic domain. Joshua Atkins (Johns Hopkins University, 853 West 35th Street, Baltimore, MD 21211, USA, joshatkins@jhu.edu), James West (Johns
Hopkins University, Department of Electrical Engineering, 3400 North Charles Street, Baltimore, MD 21218, USA, jimwest@jhu.edu)

The problem of creating a multiuser hands-free immersive telecommunications environment poses many challenges for acoustic signal processing. The most pressing is the creation of a fast multichannel acoustic echo canceller (MCAEC) to eliminate acoustic feedback created in the speakermicrophone loop. Traditional multichannel adaptive algorithms for echo cancellation are not fast enough to work in systems with highly correlated multichannel signals like those found in the traditional telecommunications setup. This work presents analysis of a proposed acoustic echo cancellation system that operates in the spherical harmonic domain. Transforming the microphone and speaker signals into the spherical harmonic domain reduces both the number of channels and the correlation between channels for faster convergence of the adaptive MCAEC filters. This method also has the added benefit of being independent of the microphone and speaker array configuration, resulting in a hybrid mono/stereo/multichannel acoustic echo canceller. This work is funded by NSF Grant IIS-0534221.

\section{$1: 40$}

1pEAb3. Improving the directivity of a Soundfield microphone using least-squares estimation. Craig Jin (University of Sydney, School of Electrical and Information Engineering, Bldg. J03, 2006 Sydney NSW, Australia, craig@ee.usyd.edu.au), Alan Kan (University of Sydney, School of Electrical and Information Engineering, Bldg. J03, 2006 Sydney NSW, Australia, alan@carlab.ee.usyd.edu.au), Andre Van Schaik (University of Sydney, School of Electrical and Information Engineering, Bldg. J03, 2006 Sydney NSW, Australia, andre@ee.usyd.edu.au)

Faller [1] has recently shown that highly directive microphone systems can be obtained using coincident microphones and appropriate signal processing to separate direct and ambient sound. We apply this approach to a Soundfield microphone in which coincident virtual first-order microphone patterns pointing in any direction are available. The variation with our ap- 
proach is that we apply a specific gain pattern across a set of coincident cardiod or hypercardiod microphones to determine the pick-up direction. A least-squares estimate is made of the spectrum of the on-axis sound. We empirically investigate the performance of this directive microphone technique. [1] C. Faller, "A highly directive 2-capsule based microphone system," in Proc. of 123rd Audio Engineering Convention, New York, U.S.A., October $5-8,2007$

\section{2:00}

1pEAb4. A comparison of measured and theoretical performance of a co-centred rigid and open spherical microphone array. Craig Jin (University of Sydney, School of Electrical and Information Engineering, Bldg. J03, 2006 Sydney NSW, Australia, craig@ee.usyd.edu.au), Abhaya Parthy (University of Sydney, School of Electrical and Information Engineering, Bldg. J03, 2006 Sydney NSW, Australia, aparthy@it.usyd.edu.au), Andre Van Schaik (University of Sydney, School of Electrical and Information Engineering, Bldg. J03, 2006 Sydney NSW, Australia, andre@ee.usyd.edu.au)

We present a comparison of the measured and theoretical performance of a dual co-centred spherical microphone array that consists of an open spherical microphone array with a smaller, rigid spherical microphone array at its centre. The dual co-centred spherical microphone array has 64 microphones, with 32 microphones on the open spherical microphone array of radius 6.30 $\mathrm{cm}$ and 32 microphones on the rigid spherical microphone array of radius $1.63 \mathrm{~cm}$. We have previously shown [1] that this even distribution of microphones, between the two spherical microphone arrays, provides a greater frequency range of operation for a third-order, 64-channel spherical microphone array compared to a single rigid 64-channel spherical array. The performance of the dual co-centred spherical microphone array is measured in an anechoic chamber using a speaker mounted on a robotic arm. A comparison is made between the theoretical and measured directivity pattern for various frequencies. [1] A. Parthy, C. Jin, and A. van Schaik "Optimisation of Co-centred Rigid and Open Spherical Microphone Arrays," in Proc. of 120th Audio Engineering Convention, Paris, France, May 20-23, 2006.

\section{2:20}

1pEAb5. Towards 'not so spherical' microphone arrays. Nicolas Epain (France Telecom R\&D, 2 avenue Pierre Marzin, 22300 Lannion, France, nicolas.epain@orange-ftgroup.com), Jérôme Daniel (France Telecom R\&D, 2 avenue Pierre Marzin, 22300 Lannion, France, jerome.daniel @ orange-ftgroup.com)

An increasing number of applications require that the sound field spatial properties are accurately described. Using arrays consisting of omnidirectional microphones distributed at the surface of a rigid sphere has been proven to be an efficient yet practical strategy to obtain such three dimensional sound field descriptions. However these sensor arrays are known to suffer from frequency limitations, mainly due to the sphere directivity properties. In this paper, it is proposed to optimize the shape of the object upon which the microphones are located, in order to make them more directive. Results of measurements and simulations show that such "not so spherical" shapes could help at widening the microphone array frequency range.

\section{2:40}

1pEAb6. Global synthesis of superdirective frequency-invariant beam patterns. Marco Crocco (DIBE - Dept. of Biophysical and Electronic Engineering - University of Genoa, Via Opera Pia 11a, 16145 Genova, Italy, crocco@dibe.unige.it), Stefania Repetto (DIBE - Dept. of Biophysical and Electronic Engineering - University of Genoa, Via Opera Pia 11a, 16145 Genova, Italy, stefania@dibe.unige.it), Andrea Trucco (DIBE - Dept. of Biophysical and Electronic Engineering - University of Genoa, Via Opera Pia 11a, 16145 Genova, Italy, trucco@ieee.org)

Frequency-invariant beam patterns are often required by systems using an array of sensors to process broadband signals. If the spatial aperture is shorter than the involved wavelengths, the use of a superdirective beam pattern is essential to attain an efficient system. In this context, robustness to array imperfections is a crucial feature. In the literature, only a few approaches have been proposed to design a robust, superdirective, frequency- invariant beamformer, based on a filter and sum architecture: in all of them, the frequency invariance is achieved imposing an a priori desired beam pattern. However the choice of a suitable desired beam pattern is not trivial and depends on the specific design case: an improper selection of the desired beam pattern can produce unsatisfactory performances. We propose a new method of global synthesis, computationally inexpensive, allowing to design a robust broadband beam pattern with an optimal trade-off between the frequency invariance and the directivity, without the need of imposing a priori a desired beam pattern. The results show that the synthesized beam patterns have a directivity, a frequency-invariance, and a robustness that are very similar to or better than those of the beam patterns obtained by the literature methods.

\section{3:00}

1pEAb7. The generation of binaural signals from a regularly sampled soundfield using a beamforming approach. Mark Poletti (Industrial Research Ltd., PO Box 31-310, 310 Lower Hutt, New Zealand, M.Poletti@irl.cri.nz), Peter Svensson (Norwegian Univ. of Science and Technology, O.S. Bragstads plass 2B, Dept. of Electronics and Telecommunications, NO-7491 Trondheim, Norway, svensson@iet.ntnu.no)

Auditorium designs can be evaluated prior to construction by numerical modeling of the design. Subjective assessment of the design requires auralization of the computed soundfield at a desired listener position and the binaural format is dominant for representation of the soundfield. For high accuracy the modeling is based on solving the wave equation numerically, which typically produces the sound pressure on a rectangular grid. Such a sampled soundfield is not straightforward to convert to a binaural format. This paper investigates binaural conversion from the sound pressure at a selected number of grid points using a least squares beamforming approach. Low-frequency axisymmetric emulations are derived assuming a solid sphere model of the head, and an open spherical array of 640 microphones is used to emulate ten measured HRTF data sets from the CIPIC database for half the audio bandwidth. The microphone positions might be displaced from the exact spherical surface to fit the mesh sample points. The spherical array can produce high-accuracy band-limited emulation of any human subject's measured HRTFs for a fixed listener position by using individual sets of beamforming impulse responses.

\section{3:20}

1pEAb8. New measurements of head-related transfer functions with an optimized ear-canal microphone. Christopher Haut (Oldenburg University, Institute for Physics, 26111 Oldenburg, Germany, christopher@aku.physik.uni-oldenburg.de), Jacqueline Rausch (Oldenburg University, Institute of Physics - Acoustics, Carl-von-Ossietzky Str. 9-11, $26111 \quad$ Oldenburg, Germany, jacqueline@aku.physik.uni-oldenburg.de), Volker Mellert (Oldenburg University, Institute for Physics, 26111 Oldenburg, Germany, volker.mellert @ uni-oldenburg.de)

Affecting the ear-canal impedance by inserting a microphone produces considerable changes in the directional characteristics of the ear with increasing frequency. Head-related transfer functions (HRTFs) are measured in the horizontal and median plane under systematic change of the ear-canal microphone more or less blocking the ear-canal entrance. A microphone arrangement is developed which minimizes the influence on the given impedance of the ear canal entrance. New measurements of HRTFs are reported and compared with well-known literature results. The "undisturbed" HRTFs serve as objective functions for a microphone array which substitutes the dummy head in head-related (stereophonic) recordings. The (complex) objective functions are modified for the microphone array according to the relevance in directional hearing. 


\section{3:40}

1pEAb9. Study of a concert harp's radiation using acoustic imaging methods. Quentin Leclere (Laboratoire Vibrations Acoustique - INSA Lyon, 25 bis avenue Jean Capelle, Bâtiment Saint-Exupéry, F-69621 Villeurbanne cedex, France, quentin.leclere@insa-lyon.fr), Jean-Loic Le Carrou

(Laboratoire d'Acoustique de l'Université du Maine, Avenue Olivier Messiaen, 72085 Le Mans, France, jean-loic.le_carrou@univ-lemans.fr), François Gautier (Laboratoire d'Acoustique de l'Université du Maine, Avenue Olivier Messiaen, 72085 Le Mans, France, francois.gautier@univlemans.fr)

Recent studies have been conducted to understand the low frequency radiation of a concert harp. Experiments have been carried out in a semianechoic room: the harp's soundboard is excited with a shaker and the pres- sure is measured at more than 600 positions on a nearly hemispherical surface at a distance of about $2 \mathrm{~m}$ from the instrument. Simple source models with a set of monopoles have been optimized to fit the measured acoustic radiation. These models are very satisfactory in the low frequency domain but cannot properly reproduce the measured field above $350 \mathrm{~Hz}$. The aim of this paper is to present the application of acoustic imaging tools to this academic case: volumetric velocity and acoustic power maps in the harp plane are computed with an optimized beamforming and with a regularized inverse FRF method up to $1 \mathrm{kHz}$. The optimization of the beamforming is realized using a variable windowing factor, and the regularization of the inverse FRF method with a Tikhonov approach. The presented results permit the evaluation of the importance of the bass reflex effect in the harp, of the contribution of the first bending modes and of the contribution of the soundbox vibrations in the radiated sound field.

MONDAY AFTERNOON, 30 JUNE 2008

ROOM 353, 4:20 TO 7:40 P.M.

\title{
Session 1pEAc
}

\section{Engineering Acoustics and Psychological and Physiological Acoustics: Hearing Aid Engineering I}

\author{
Daniel M. Warren, Cochair \\ Knowles Electronics, 1151 Maplewood Dr, Itasca, IL 60143, USA \\ Stefan Launer, Cochair \\ Phonak AG, Laubisrütistr. 28, CH-8712 Stäfa, Switzerland
}

Invited Paper

4:20

\begin{abstract}
1pEAc1. Model-based signal processing for hearing aids. Volker Hohmann (Carl von Ossietzky Universität Oldenburg, Ammerländer Heerstraße 114-118, 26111 Oldenburg, Germany, volker.hohmann@uni-oldenburg.de)

The aim of the work presented in this talk is to investigate potential applications of models of human auditory processing to signal processing in hearing aids. In particular, it is shown that auditory models may be used as objective indicators of the perceptual quality of single- and multichannel noise reduction strategies. Furthermore, a general framework for computational auditory scene analysis (CASA) is introduced and applied to the problem of estimating spatial direction and spectral envelope of super-imposed sound sources from a binaural input signal. Finally, implications of using nonlinear peripheral models for the design of compression systems in hearing aids are discussed. In particular, results from a multiband instantaneous compression scheme are presented, which is based on a novel nonlinear auditory filterbank controlled by the instantaneous frequency calculated in frequency sub-bands.
\end{abstract}

\section{Contributed Paper}

\section{4:40}

1pEAc2. The sound field in life-size replicas of human ear canals occluded by a hearing aid. Mike R. Stinson (Inst. for Microstructural Sciences, National Research Council of Canada, Bldg. M-36, Ottawa, ON K1A 0R6, Canada, mike.stinson@nrc-cnrc.gc.ca), Gilles A. Daigle (Inst. for Microstructural Sciences, National Research Council of Canada, Bldg. M-36, Ottawa, ON K1A 0R6, Canada, gilles.daigle@nrc-cnrc.gc.ca)

In addition to the longitudinal sound pressure distributions that form in the human ear canal, large transverse variations can arise in the vicinity of an occluding hearing aid. These effects are being studied, numerically and experimentally, making use of life-size ear canal replicas. Using digital representations of real ear canal geometries [Stinson and Lawton, J. Acoust.
Soc. Am. 85, 2492-2503 (1989)], a polyjet fabrication process forms replicas with a spatial accuracy of better than $0.1 \mathrm{~mm}$. A hearing aid test fixture, with vent, receiver, and an inner microphone, occludes the replica canal and provides the acoustical input. The sound field inside the canal is measured using a $0.2 \mathrm{~mm}$ o.d. probe microphone. In parallel, the interior sound field is calculated using a boundary element method, using the same ear canal geometry as the replicas and accounting for the acoustical boundary conditions presented by the eardrum and the hearing aid. In the current series of ear canal replicas, the eardrum is rigid. Measurements and calculations are in good agreement. Large transverse variations of sound pressure level, as much as $20 \mathrm{~dB}$ at $8 \mathrm{kHz}$, are observed across the inner face of the hearing aid, particularly near the receiver and vent ports. 


\section{Invited Papers}

\section{5:00}

1pEAc3. Acoustics of open fittings. Matthias Blau (Institut für Hörtechik + Audiologie, FH OOW, Ofener Str. 16, D-26121 Oldenburg, Germany, matthias.blau@fh-oldenburg.de), Tobias Sankowsky (Institut für Hörtechik + Audiologie, FH OOW, Ofener Str. 16, D-26121 Oldenburg, Germany, tobias.sankowsky@fh-oldenburg.de), Alfred Stirnemann (Phonak AG, Laubisrütistr. 28, CH-8712 Stäfa, Switzerland, alfred.stirnemann@phonak.ch), Hannes Oberdanner (Phonak AG, Laubisrütistr. 28, CH-8712 Stäfa, Switzerland, hannes.oberdanner@phonak.ch), Nicola Schmitt (Phonak AG, Laubisrütistr. 28, CH-8712 Stäfa, Switzerland, nicola.schmitt@phonak .ch)

The use of so-called "open fittings" instead of individual ear shells has become very popular in hearing aid fitting, in particular because the open fittings avoid the occlusion effect, but also because they are more comfortable, easier to manufacture, and cosmetically preferred. On the other hand however, there are acoustical issues with open fittings, including the mixture of direct and amplified sound, a poor low-frequency performance, an increased risk of feedback and a supposedly less reproducible position of the sound delivering device (tubing or speaker) in the ear canal, which in turn may result in a greater variability of acoustic parameters such as RECD and REOG. The two latter issues are addressed here in a study with 20 subjects, for individual shell and a number of open fittings, comprising closed and open domes of different diameters with tubings as well as with ear canal receivers. It was observed that in comparison to individual shell fittings, the open fittings did not exhibit higher interindividual differences of RECD and REOG, but up to 15 $\mathrm{dB}$ (closed domes) to $25 \mathrm{~dB}$ (open domes) lower feedback thresholds.

\section{$5: 20$}

1pEAc4. Estimation of maximum stable gain in a hearing aid. Ivo L. Merks (Starkey Laboratories, 6600 Washington Ave S, Eden Prairie, MN 55344, USA, ivo_merks@ @starkey.com), Karrie Recker (Starkey Laboratories, 6600 Washington Ave S, Eden Prairie, MN 55344, USA, karrie_recker@starkey.com), Harikrishna P. Natarajan (Starkey Laboratories, 6600 Washington Ave S, Eden Prairie, MN 55344, USA, harikrishna_natarajan@starkey.com), Lalin S. Theverapperuma (Starkey Laboratories, 6600 Washington Ave S, Eden Prairie, MN 55344, USA, lalin_theverapperuma@starkey.com)

Feedback cancellation (FBC) algorithms have become an important part of hearing aids, allowing the Maximum Stable Gain (MSG) to be increased by up to $25 \mathrm{~dB}$ beyond what is possible without an FBC. Although FBC algorithms have improved the usability of hearing aids, it is difficult to predict the MSG for a given individual with a particular hearing aid. Knowledge of this information would enable the audiologist to make more informed decisions regarding the appropriateness of a hearing aid/earmold, and it could be used to counsel the patient regarding feedback. A method of estimating the MSG of a hearing aid with and without an FBC will be presented. The method uses a finite impulse response filter to approximate the acoustic path. The filter coefficients are estimated during the initialization of the FBC. From these filter coefficients, the MSG for the hearing aid with and without FBC are calculated. This method has been implemented in the firmware and fitting software. Using a variety of device styles, microphone modes and feedback paths, it has been verified that the estimated MSG matches the actual MSG within $6 \mathrm{~dB}$ for $90 \%$ of hearing aids.

\section{5:40}

1pEAc5. "Auditory scene analysis" in hearing instruments. Matthias Froehlich (Siemens Medical Solutions SAT, 91058 Erlangen, Germany, Matthias.froehlich@ siemens.com)

The term "auditory scene analysis" generally refers to a categorization of a given acoustic situation based on the acoustic signal only, where the results determine the subsequent processing of the acoustic signal within some auditory context. According to this definition, several approaches can be differentiated in the field of hearing instrument development. They differ in the computational complexity of the particular analysis methods applied, as well as in the subsequent action. Some of these approaches have been realized in commercially available hearing instruments, others lie still ahead. A simple example of the former category is noise reduction algorithms that address different classes of noises, examples of the latter are MPEG4-like virtual arrangements of media objects. In the presentation, different approaches will be discussed in terms of potential benefit and technical realization, as well as their limitations. For approaches already realized in commercially available hearing instruments, the expected benefit will be aligned with results from clinical studies.

\section{Contributed Paper}

\section{6:00}

1pEAc6. Prediction of perceived sound quality of hearing aids (algorithms) using perceptual models. Rainer Huber (Kompetenzzentrum HörTech, Marie-Curie-Str. 2, 26129 Oldenburg, Germany, Rainer.Huber@HoerTech.de)

This contribution presents an overview of basic approaches for predicting the perceived sound quality of hearing aids and hearing aid algorithms using auditory processing models for hearing impaired. Comparison-based concepts will be considered in particular. The main characteristic of these concepts is the comparison of internal representations (the outputs of the auditory models) of a test and a reference signal. While this approach is straight-forward and has proven to be successful for the prediction of sound quality of lossy speech and audio processing systems perceived by normalhearing listeners, the requirement of a reference representing the optimal quality can pose a problem in the case of hearing aids and hearing-impaired listeners. The potential and limitations of comparison-based approaches will be illustrated by example results from different studies obtained with an extended version of the perceptual audio quality model PEMO-Q. 


\section{Invited Papers}

\section{6:20}

1pEAc7. Real-world effectiveness of directional microphone hearing aids. Ruth Bentler (University of Iowa, 250 Hawkins Drive, Wendell Johnson Clinic, Iowa City, IA 52242, USA, ruth-bentler@uiowa.edu)

Single, dual, triple, and array microphone designs that are static, automatic and/or adaptive are now available in marketed hearing aids. The cost of designing and producing these more sophisticated systems is significant; yet, studies of user benefit are often equivocal, particular those involving self-report or field-obtained measures. In a series of studies we have attempted to characterize (model) the function of a number of these designs in simple and complex environments in an effort to explain the results obtain in both laboratory and field studies. Rather than directivity limitations, factors such as internal noise, loudness preference and visual cues have all been implicated in the limited user benefit, and will be discussed in this presentation.

\section{6:40}

1pEAc8. Whispering in your ear: a recent history of subminiature transducers. Daniel M. Warren (Knowles Electronics, 1151 Maplewood Dr, Itasca, IL 60143, USA, daniel.warren@knowles.com)

For 50 years, the history of hearing aid transducer development could be read in a simple photograph and timeline, where the size of the transducer was inversely correlated to the year it was developed. This size curve corresponds to hearing aid development over the same time period, progressing from body-worn aids in the 1940s to large behind-the-ear (BTE), smaller BTE's, in-the-ear (ITE), and successively deeper in- the-canal (ITC) devices through the 1990s. Although size still matters, in the last decade there has been less effort in reducing the size of hearing aids and more on increasing features, usability, and comfort. Development of hearing aid transducers has become similarly multidimensional. This paper charts the progress from the linear smaller-is-better design model to lower vibration, higher acoustic output, lower current draw, reduced radio frequency interference, wider bandwidth, and other multifaceted challenges of modern hearing aid transducer design.

\section{Contributed Papers}

7:00

1pEAc9. High sound quality and concha headphones: where are the limitations? Lola Blanchard (Bang\&Olufsen ICEpower/DTU, Gl. Lundtoftevej 1b, st., 2800 Lyngby, Denmark, lob@bang-olufsen.dk)

Concha headphones (the small type of headphone that rests at the entrance of the ear canal) are the most popular type of headphones for everyday use. However, the sound quality produced by even "high quality" concha headphones is rather poor. This paper gives an overview of the different factors that affect the quality of this type of headphones, describes the measurement difficulties, and presents a model. The leakage between the headphone and the ear changes every time the headphone is inserted in the ear. This alters the perceived sound quality, especially at low frequencies, and makes measurements very difficult. Moreover, because of energy efficiency considerations, a typical transducer design is prone to nonlinear behaviour, and a high distortion rate can be measured for most concha headphones. The measured transducer parameters and leakage have been implemented in a model realised in Simulink. The simulation model, which makes it possible to study the influence of the various parameters including the uncontrolled leakage, is the first step towards developing a compensation technique.

$7: 20$

1pEAc10. A two-stage binaural speech enhancement approach for hearing aids with preserving binaural benefits in noisy environments Junfeng Li (Japan Advanced Institute of Science and Technology, 1-1,
Asahidai, Nomi, 923-1292 Ishikawa, Japan, junfeng@jaist.ac.jp),Shuichi Sakamoto (R.I.E.C., Tohoku University, 2-1, Katahira, Aoba-ku, 980-8577 Sendai, Japan, saka@ais.riec.tohoku.ac.jp), Satoshi Hongo (Faculty of Design and Computer Applications, Miyagi National College of Technology, 48, Nodayama, Medeshima Shiote, 981-1239 Natori, Japan, hongo@miyagi-ct.ac.jp), Masato Akagi (Japan Advanced Institute of Science and Technology, 1-1, Asahidai, Nomi, 923-1292 Sendai, Japan, akagi@jaist.ac.jp), Yôiti Suzuki (R.I.E.C., Tohoku University, 2-1, Katahira, Aoba-ku, 980-8577 Sendai, Japan, yoh@ais.riec.tohoku.ac.jp)

Speech enhancement is one of the most crucial functions, if not the most, in hearing aids, as hearing impaired people have great difficulty in understanding speech in noisy environments. In this paper, we propose a two-stage binaural speech enhancement approach for hearing aids, which consists of interference estimation by pre-trained adaptive filters and speech enhancement using the Wiener filters. Main attention is then paid to the theoretical analysis of this system and the experimental comparisons with the traditional binaural speech enhancement approaches. The comparisons are conducted with the following two considerations: interference suppression performance and the ability in preserving binaural cues which give birth to listener's own "binaural gain." We finally give the general discussion on this proposed binaural speech enhancement algorithm from the viewpoints of theory, through implementation, to evaluation. 


\title{
Session 1pEAd
}

\section{Engineering Acoustics, Underwater Acoustics, and ECUA: Sonar Transducer Design and Modeling I}

\author{
John B. Blottman, Chair \\ Naval Undersea Warfare Center, Division Newport, 1176 Howell Street, Newport, RI 02841-1708, USA
}

\section{Invited Papers}

$5: 40$

1pEAd1. Advanced application-motivated design techniques for high performance piezocomposite underwater sonar transducers and arrays. Sandy Cochran (Institute for Medical Science and Technology, University of Dundee, Wilson House, 1 Wurzburg Loan, DD2 1FD Dundee, UK, s.cochran@dundee.ac.uk), Christine E. Demore (Institute for Medical Science and Technology, University of Dundee, Wilson House, 1 Wurzburg Loan, DD2 1FD Dundee, UK, c.demore@ dundee.ac.uk), Pablo Marin (Piezo Composite Transducers Ltd, Aberdeen Science Park, Balgownie Drive, Bridge of Don, AB22 8GU Aberdeen, UK, pablo.marin@pct-ltd.co.uk), Richard Marsh (Tritech International Ltd, Peregrine Road, Westhill Business Park, Westhill, AB32 6LJ Aberdeen, UK, richard-marsh@tritech.co.uk), Keith Mayne (Piezo Composite Transducers Ltd, Aberdeen Science Park, Balgownie Drive, Bridge of Don, AB22 8GU Aberdeen, UK, keith.mayne@pct-ltd.co.uk), Graeme McRobbie (University of the West of Scotland, High Street, PA1 2BE Paisley, UK, graeme.mcrobbie@uws.ac.uk), Michelle Wallace (Doosan Babcock Energy, Porterfield Road, PA4 8DJ Renfrew, UK, mwallace@doosanbabcock.com), Mark P. Walsh (Piezo Composite Transducers Ltd, Aberdeen Science Park, Balgownie Drive, Bridge of Don, AB22 8GU Aberdeen, UK, mark.walsh@pct-ltd.co.uk), Richard Wright (Tritech International Ltd, Peregrine Road, Westhill Business Park, Westhill, AB32 6LJ Aberdeen, UK, dick-wright@tritech.co.uk)

Developments in areas as diverse as advanced piezoelectric materials and low cost digital electronics continue to offer new possibilities to the designer of high performance underwater sonar transducers and arrays. Many of these are likely to be realised with complex composite materials comprising a polymer matrix with rods of piezoelectric ceramic such as PZT $5 \mathrm{H}$ or crystal such as PMN-PT which require advanced design techniques. This paper first briefly reviews relevant capabilities of conventional equivalent circuit and wave equation transducer models, and the composite homogenisation technique of Smith and Auld. The increasing possibilities offered by finite element analysis (FEA) running on relatively low-cost computing hardware are also considered. Whichever design technique is adopted, accurate material properties are crucial and this is reported through reference to a range of piezoelectric and passive materials. The potential variation of such properties across the operating temperature range of underwater transducers is also considered, illustrated with experimental measurements. As understanding of high performance transducer structures grows, future possibilities also become evident, such as the 3-1 connectivity multilayer composite. The paper is therefore completed with an outline of these complex structures and a stochastic mathematical technique that can be used to optimise their designs automatically.

\section{6:00}

1pEAd2. Leveraged motion underwater sound piston transducers. John Butler (Image Acoustics, Inc., 97 Elm Street, Cohasset, MA 02025, USA, jbutler@imageacoustics.com), Alexander L. Butler (Image Acoustics, Inc., 97 Elm Street, Cohasset, MA 02025, USA, abutler@imageacoustics.com)

Flextensional transducers use leveraged shell motion to produce enhanced acoustic radiation from compact sources, which are often used in low frequency underwater sound applications. The most commonly used flextensional is the Class IV where an oval shell is driven along its major axis by a stack of piezoelectric ceramic elements. We present here a review of a new class of leveraged transducers in which the shell motion drives pistons attached to the shell at the location of maximum magnified motion. Because of the uniform motion of the pistons, greater source strength and radiation load is attained compared to the tapered motion of flextensional shells. The leveraged motion typically yields a motion magnification of approximately three and an impedance load magnification of approximately nine, resulting in reasonably low $\mathrm{Q}$ for a compact low frequency transducer. Various designs are presented and illustrated including one in which the piezoelectric drive simultaneously acts as a tail mass and one which operates in a high impedance velocity control mode under array conditions. Lumped models as well as FEA models and measured results are presented to illustrate the concept and performance of PZT and PMN-PT driven designs. [Work supported in part by ONR.]

\section{6:20}

1pEAd3. Electro-active polymer transduction for distributed netted sensing. Dennis F. Jones (Defence R\&D Canada - Atlantic, P.O. Box 1012, Dartmouth, NS B2Y 3Z7, Canada, dennis.jones@drdc-rddc.gc.ca), John B. Blottman (Naval Undersea Warfare Center, Division Newport, 1176 Howell Street, Newport, RI 02841-1708, USA, blottmanjb@npt.nuwc.navy.mil), Roger T. Richards (Naval Undersea Warfare Center, Division Newport, 1176 Howell Street, Newport, RI 02841-1708, USA, richardsrt@npt.nuwc.navy.mil)

The Naval Undersea Warfare Center in Newport, RI is developing an electro-active polymer-based sensing node for use in a persistent distributed underwater surveillance system. Persistence demands extreme energy conservation measures. The node will resemble a jellyfish in form, complete with tentacles housing a volumetric array of light-weight piezoelectric polyvinylidene fluoride trifluoroethylene (PVDF-TrFE) copolymer cylindrical hydrophones designed for an ultra-low power acoustic receiver. Each cylinder has con- 
ductive silver electrodes on the inside and outside surfaces, a length of $2.5 \mathrm{~cm}$, an outside diameter of $11 \mathrm{~mm}$, a $1 \mathrm{~mm}$ wall thickness, and a mass less than $2 \mathrm{~g}$. Operating in the hydrostatic mode, the hydrophone sensitivity is typically $-195 \mathrm{~dB} / / 1 \mathrm{~V} / \mu \mathrm{Pa}$ and is stable with both hydrostatic pressure (50 to $1000 \mathrm{psi})$ and temperature $\left(-1\right.$ to $\left.35^{\circ} \mathrm{C}\right)$. [Work supported by the Office of Naval Research.]

\title{
6:40
}

1pEAd4. Magnetostrictive compacted sonar transducer design. Stephen C. Butler (Naval Undersea Warfare Center, 1176 Howell St, Newport, RI 02841, USA, butlersc@npt.nuwc.navy.mil), Julie Slaughter (Etrema Products, Inc., 2500 N. Loop Drive, Ames, IA 50010, USA, julie.slaughter@etrema.com)

A compact modular high power magnetostrictive sound source that is capable of producing scanning Cardioid beams every 45 degrees in the horizontal plane and operates over one octave has been fabricated. The device consists of 8 transducer elements in the form of a ring that radiates acoustic energy radially outward. This design uses the operation principles of a peizoceramic transducer of similar design [J. L. Butler and A. L. Butler, J. Acoust. Soc. Am. 119, 3409 (2006)], which operated in a higher frequency band. The sound source can be driven to form omni-directional, dipole, and quadrupole beam patterns. By combining the measured electrical drive and acoustic pressure amplitude and phase coefficients of these beam patterns a narrower type Cardioid beam pattern is generated. The transducer elements are of a tonpilz type vibrator consisting of magnetostrictive Terfenol-D drive rods sandwiched between radiating head mass and a tail mass that is common to all elements with tie bolts consolidating the parts together. The Terfenol-D rods are interlaced with rare earth magnets to provide the DC magnetic bias field for the AC drive field provided by a coil. FEA models using ATILA and COMSOL are used for structural and magnetic analysis predictions of the device. [Work supported by ONR.]

$$
\text { 7:00 }
$$

1pEAd5. Broadband tonpilz transducers based on single crystal relaxor ferroelectrics: Design and modeling. Richard J. Meyer (The Applied Research Laboratory/ The Pennsylvania State University, P.O. Box 30, State College, PA 16801, USA, rmeyer@psu.edu), Douglas C. Markley (The Applied Research Laboratory/ The Pennsylvania State University, P.O. Box 30, State College, PA 16801, USA, dcm13@psu.edu)

Very broad bandwidth transduction capability has been made possible by high coupling piezoelectric single crystal materials. Recent work has shown that PMN-PT based tonpilz elements with a single resonance have a device electromechanical coupling coefficient greater than 0.86 and can easily achieve more than two octaves of source level bandwidth. Implementing single crystal based tonpilz elements reduces array packaging volume and weight while maintaining source level. In addition, high coupling projectors reduce transmit system complexity when compared to traditional materials. This paper will describe approaches to creating broadband SONAR projectors using single crystal materials. Crystal anisotropy plays a key role in transducer design. This anisotropy requires final designs to be modeled in three dimensions. ATILA finite element code was used to capture all of the modes of vibration and performance predictions. Motor section geometries and material compositions will be compared. The model predictions will also be compared to measured data.

\section{Session 1pMUa}

\section{Musical Acoustics: Acoustic Measurements on Wind Instruments I}

\author{
Noam Amir, Cochair \\ Tel Aviv University, Dept. of Communications Disorders, Sheba Medical Center, Tel Hashomer, 52621, Israel \\ David B. Sharp, Cochair \\ Acoustics Research Group, DDEM, MCT Faculty, Open University, Walton Hall, Milton Keynes, MK7 6AA, UK
}

\section{Invited Papers}

\author{
1:00
}

1pMUa1. A new impedance sensor for wind instruments. Jean-Pierre Dalmont (Laboratoire d'Acoustique de l'Université du Maine, Avenue Olivier Messiaen, 72085 Le Mans, France, Jean-Pierre.Dalmont@univ-lemans.fr), Jean Christophe Le Roux (CTTM, 20, rue Thales de Milet, 72000 Le Mans, France, jcleroux@cttm-lemans.com)

Our aim was to build a low cost but accurate portable impedance sensor. The adopted technique is the one using a source with a back cavity. A first elctret microphone on the front of the source measures the pressure at the input of the instrument weather a second one measures the pressure in the cavity, which is proportional to the volume flow supplied by the source. By choosing a sufficiently small sealed cavity and a small piezoelectric source the system do not exhibit any cut-off frequency in the measurement range. The calibration is then simplified and only few parameters are needed to model the three complex calibration functions. This calibration is performed with three nonresonant calibration loads. Moreover, it is shown that the geometry of the sensor being known, the measurement with a rigid wall is sufficient to calibrate the sensor. Results show that it is possible to achieve a measurement with an accuracy lower than $1 \mathrm{~dB}$ in the range 50-4000 $\mathrm{Hz}$. 
1pMUa2. Input impedance measurements of conical acoustic systems using the two-microphone technique. Antoine Lefebvre (Schulich School of Music, McGill University, 555 Sherbrooke Street West, Montreal, QC H3A 1E3, Canada, antoine.lefebvre2@mail.mcgill.ca), Gary Scavone (Schulich School of Music, McGill University, 555 Sherbrooke Street West, Montreal, QC H3A 1E3, Canada, gary@music.mcgill.ca)

The two-microphone technique with a broadband excitation has been used for the measurement of various objects including straight conical waveguides and alto saxophones. An improved procedure has been developed whereby the noise excitation signal is pre-filtered by the inverse frequency response of the system under consideration. This approach helps reduce distortion and improves the SNR of the measurement. Results with and without pre-filtering are compared for different fingerings of an alto saxophone. The input impedance of a straight conical waveguide is compared with theory and discrepancies are analyzed. The input impedance of saxophones are evaluated with the transmission matrix approach and compared with measurements. A software environment for efficient comparison and analysis of measurement data with theoretical calculations is presented. We also propose a framework for sharing raw measurement data among researchers to allow comparison of results obtained with different apparatus on similar objects to better quantify their accuracy.

\title{
Contributed Paper
}

\section{$1: 40$}

1pMUa3. Improved precision in acoustic impedance measurements by using calibration loads without resonances. Paul Dickens (University of New South Wales, Music Acoustics, School of Physics, NSW 2052 Sydney, Australia, PDickens@resmed.com.au),John Smith (University of New South Wales, Music Acoustics, School of Physics, NSW 2052 Sydney, Australia, john.smith@unsw.edu.au), Joe Wolfe (University of New South Wales, Music Acoustics, School of Physics, NSW 2052 Sydney, Australia, J.Wolfe@unsw.edu.au)

Although the input impedance of a one-dimensional waveguide is simply defined by the ratio of pressure to volume flow measured at its input, the deficiencies of available transducers require a more complicated approach for precise measurements. These can involve multiple transducers at points within an impedance head connected to the measured load. However, reso- nances and/or singularities during calibration and/or measurement often limit the precision of acoustic impedance spectra. This paper reviews and compares several established techniques, and describes a technique that incorporates three features that can considerably improve precision. The first feature involves minimising the problems due to resonances by calibrating the instrument using up to three different acoustic reference impedances that do not themselves exhibit resonances: an acoustically infinite waveguide, an acoustic open circuit and an acoustically infinite flange. The second feature involves using multiple pressure transducers to reduce the effects of measurement singularities. The third involves iteratively tailoring the spectrum of the stimulus signal to control the distribution of errors across the particular measured impedance spectrum. Examples are given of the performance of the technique on simple cylindrical waveguides and some wind instruments, including the saxophone and clarinet.

\section{Invited Paper}

\begin{abstract}
2:00
1pMUa4. A practical way to measure intonation quality of woodwind instruments using standard equipment without custom made adapters. Wilfried Kausel (Inst. f. Wiener Klangstil, Univ. f. Music, Anton von Webernplatz 1, A-1030 Vienna, Austria, kausel@mdw.ac.at), Helmut Kuehnelt (Inst. f. Wiener Klangstil, Univ. f. Music, Anton von Webernplatz 1, A-1030 Vienna, Austria, Helmut.Kuehnelt@arsenal.ac.at)

Woodwinds are not much different from brass instruments in how intonation is determined by air column resonances. Nevertheless, it is easier to measure input impedance of brasses because the input cross-section at the mouthpiece rim is circular, plane and big enough to be easily coupled to standard measuring heads. In reed instruments the input cross-section is neither flat nor circular nor well defined. Flute instruments not even have any definable input cross-section as this is controlled by the player's lower lip. On top of that, flutes are played at an open end where some coupled impedance considerably influenced by the nearby environment like lips, mouth, and face of the player has to be taken into account. Existing approaches to measure input impedance of woodwind instruments usually require custom made adaptors optimised to yield nearly accurate intonation results for a specific instrument and playing range only. The proposed new approach is to separate instrument head or mouthpiece from the body and measure both parts from their cylindrical ends. During the measurement a natural playing condition can be simulated at the now available end. Both measurements can be assembled computationally yielding useful intonation results as will be demonstrated for flutes and saxophones.
\end{abstract}

\section{Contributed Paper}

2:20

1pMUa5. Measured and calculated sounding frequencies of pipes coupled with free reeds. James Cottingham (Coe College, 1220 First Avenue NE, Cedar Rapids, IA 52402, USA, jcotting@coe.edu), Eric A. Dieckman (Truman State Univ., Physics Dept., Kirksville, MO 63501, USA, ead603@truman.edu)

The Asian free-reed mouth organs incorporate approximately symmetric free reeds coupled to pipe resonators. Previous research has shown that the reeds in these instruments behave as "blown-open" reeds in which the playing frequency is above both the natural frequency of the reed and the first peak of the measured impedance curve. Measurements of the input impedance have been made for a variety of these instruments. Detailed calculations of input impedance have also been made using transmission matrices, taking into account the position of the reed along the pipe, tuning slots, finger holes, and nonuniform cross sections. These calculations are in good agreement with the measured impedances of the same instruments. If the reed is treated as a damped, driven harmonic oscillator, the sounding frequencies of these reed-pipes can be predicted using a phase relation between the reed vibration and the phase of the complex impedance. [Research supported by National Science Foundation REU Grant PHY-0649007.] 


\section{Invited Papers}

2:40

1pMUa6. Calibration of apparatus for wave separation in wind instruments. Giovanni De Sanctis (Queen's University Belfast, University Road, BT7 1NN Belfast, UK, gdesanctis01@qub.ac.uk), Maarten Van Walstijn (Sonic Arts Research Centre, Queen’s University Belfast, BT7 1NN Belfast, UK, m.vanwalstijn@qub.ac.uk)

Knowledge of the forward and backward traveling waves inside the bore of a wind instrument is a useful starting point for the estimation of its playing parameters. These are mainly parameters describing the mouthpiece embouchure and the states of the tone holes. Due to the highly nonlinear behaviour of the excitation mechanism, a precise estimation is needed. The separation method under investigation relies on a model inversion, starting from the pressure measured by three microphones. For this reason the estimation is very sensitive to the relative positions of the microphones, and the latter must therefore be known very precisely. A geometrical measurement would not be reliable because of construction tolerances, including uncertainties about the acoustic centers of the microphones; therefore the distances are gathered through audio measurements. A number of approaches to such a calibration of the measurement system have been investigated, using a purposely constructed apparatus which will also be described.

\section{3:00}

1pMUa7. Acoustic pulse reflectometry for the measurement of horn crooks. Jonathan Kemp (Edinburgh University, 4201 JCMB, Kings Buildings, Mayfield Road, EH9 3JZ Edinburgh, UK, jonathan@ph.ed.ac.uk), John Chick (Edinburgh University, 4201 JCMB, Kings Buildings, Mayfield Road, EH9 3JZ Edinburgh, UK, john.chick@ed.ac.uk), Murray Campbell (Edinburgh University, 4201 JCMB, Kings Buildings, Mayfield Road, EH9 3JZ Edinburgh, UK, dmc@ph.ed.ac.uk), Darren Hendrie (Edinburgh University, 4201 JCMB, Kings Buildings, Mayfield Road, EH9 3JZ Edinburgh, UK, darrenhendrie@ hotmail.com)

The echo-based technique of acoustic pulse reflectometry can be used to measure tubular objects to determine the bore profile. In this paper, measurements of historic orchestral horn crooks are presented showing how the technique can help to determine the method of construction of historic crooks and can provide valuable information to manufacturers of reproduction period instruments. Comparison of the bore profile measured by pulse reflectometry and the known exit radius of the crook can be used to determine the presence of leaks. The technique is shown to be sensitive enough to find a leak in a horn crook that behaves reasonably under playing conditions and was not suspected of having a leak prior to testing.

\section{Contributed Papers}

\section{$3: 20$}

1pMUa8. Industrial applications of acoustic pulse reflectometry Noam Amir (Tel Aviv University, Dept. of Communications Disorders, Sheba Medical Center, 52621 Tel Hashomer, Israel, noama@ post.tau.ac.il), Oded Barzelay (AcousticEye LTD., 4 Harechev st., 67771 Tel Aviv, Israel, oded@acousticeye.com), Tal Pechter (AcousticEye LTD., 4 Harechev st., 67771 Tel Aviv, Israel, tal@acousticeye.com)

Acoustic pulse reflectometry (APR) has been used extensively in the study of wind instruments, for measuring input impedance, bore reconstruction, and fault detection. Industrial applications have often been mentioned in the literature, though they have barely been exploited. Academic APR systems are extremely bulky, which limits their industrial use severely. Two requirements from such an industrial system are that the source tubes be as short as possible, and that they be able to measure long objects, often much longer than most musical instruments, e.g., condenser tubes which are at least $8 \mathrm{~m}$ long. This creates difficult calibration problems and difficulties in obtaining sufficient SNR levels, especially at low frequencies. In this paper we describe a system with the necessary adaptations for industrial use. We demonstrate the problems that are encountered, and introduce solutions which we have implemented. Modifications to the deconvolution phase improve low frequency SNR, which is further improved using nonlinear filtering. Adaptive filters are then used to discriminate between a faulty and intact system. When faults are detected, they are identified using several identification algorithms based on matched filtering and local inverse scattering.
3:40

1pMUa9. Influence of the bocal on the sound of the bassoon. Johannes Baumgart (Technische Universität Dresden, Institute for Aerospace Engineering, $01062 \quad$ Dresden, Germany, johannes.baumgart@tu-dresden.de), Timo Grothe (Technische Universität Dresden, Institute for Aerospace Engineering, 01062 Dresden, Germany, timo.grothe@tu-dresden.de), Roger Grundmann (Technische Universität Dresden, Institute for Aerospace Engineering, 01062 Dresden, Germany, roger.grundmann@tu-dresden.de)

The bocal of a bassoon is characterized by geometrical parameters such as the bore, the outer contour and the wall-thickness as well as material parameters and the coating. Professional bassoonists select bocals extremely carefully and report influences on the playability and sound colour. In order to understand the underlying effects, several experiments were performed with bocals of the same bore bent in two different shapes and with two different materials. Images of a high-speed camera show a periodic motion of the bocal tip while the instrument is artificially blown. A modal analysis of the bocals was performed experimentally and numerically to investigate the dynamic behaviour of the structure. The first bending mode of the bocals is around $70 \mathrm{~Hz}$. While the material properties shift the modal frequencies less than $2 \%$, the shift due to the geometry is more than $10 \%$. The experimental program included playing experiments with professional bassoonists. The setup was fixed and the players used the same reed. For each player, slight shifts in the sound spectra could be found due to the use of different bocals. The harmonic structure showed changes in the frequency bands where the bending modes differ. 


\title{
Session 1pMUb
}

\section{Musical Acoustics: Edge Tone and Flue Pipes I}

\author{
Shigeru Yoshikawa, Cochair \\ Dept. of Acoustic Design, Kyushu University, Fukuoka 815-8540, Japan \\ Judit Angster, Cochair \\ Fraunhofer Institute for Building Physics, Nobelstrasse 12, Stuttgart, 70569, Germany
}

\section{Invited Papers}

1pMUb1. Influence of the geometrical parameters in flue instruments on the vorticity modulation near the separation points of the jet. François Blanc (Institut Jean Le Rond d'Alembert/LAM (UPMC/CNRS/Ministère Culture), 11, rue de Lourmel, 75015 Paris, France, blanc@lam.jussieu.fr), Pierre-Yves Lagrée (Institut Jean le Rond d'Alembert - FCIH team, 4 place Jussieu, 75006 Paris, France, pyl@ccr.jussieu.fr), Patricio De La Cuadra (Centro de Investigación en Tecnologias de Audio (CITA), Universidad Católica de Chile, Alameda 340, Oficina 13, Casilla 114-D Santiago, Chile, pcuadra@uc.cl), Benoit Fabre (Institut Jean Le Rond d'Alembert/LAM (UPMC/CNRS/Ministère Culture), 11, rue de Lourmel, 75015 Paris, France, fabreb@ccr.jussieu.fr)

In the making of the recorder, great care is given to the shape of the chamfers at the exit of the channel. Makers insist on the need of sharp chamfers, for a good tone quality. Recorders made with rounded chamfers present a noisy sound. On the other hand, in the flute, the channel is made by the lips of the musician, defining a rounder geometry. Previous work show that variations of the geometry of the exit of the channel has little influence on the velocity profile of the jet and on the position of the separation points. Thus, variations of the geometry are expected to affect mainly the interaction between the air jet and the acoustic field. The paper will present measurements together with direct Navier Stokes calculations for different geometrical configurations of a jet emerging in a transverse oscillating velocity field. We investigate the modulation of the vorticity of the jet shear layers by the acoustical field, in the vicinity of the separation points, in an attempt to develop a model that allows to predict the influence of the geometry.

\section{4:40}

1pMUb2. Mode transition of a flue organ pipe. Seiji Adachi (Fraunhofer Institute for Building Physics, Nobelstrasse 12, 70569 Stuttgart, Germany, seiji.adachi@ibp.fraunhofer.de), Judit Angster (Fraunhofer Institute for Building Physics, Nobelstrasse 12, 70569 Stuttgart, Germany, an@ibp.fhg.de), Andras Miklos (Fraunhofer Institute for Building Physics, Nobelstrasse 12, 70569 Stuttgart, Germany, an@ibp.fhg.de)

A flue organ pipe can be excited in various acoustic modes by changing the air pressure supplied to it. This research aims to reconstruct this behavior from the result of numerical flow simulation of a jet deflected by sound and from physical modeling simulation of the total sound production system. In the numerical flow simulation, motion of the jet in the pipe mouth was replicated: The jet emerges from a flue and travels in a space where the air oscillates laterally to the jet direction. As a result, the jet oscillates with the same frequency as the oscillation of the air i.e., sound. From the flow simulation, a model of the jet deflection was developed. This model was then used as a model of the sound source in the physical modeling simulation where not only the sound source but the resonance of the pipe is also modeled in a set of differential equations with delayed feedback. The mode transition observed in the physical modeling simulation was discussed by comparing with that experimentally observed.

1pMUb3. Frequency and phase characteristics of the edge tone. György Paál (Budapest University of Technology and Economics, P.O. Box 91, 1521 Budapest, Hungary, paal@ hds.bme.hu), István Vaik (Budapest University of Technology and Economics, P.O. Box 91, 1521 Budapest, Hungary, vaik@ hds.bme.hu)

Extensive experimental and computational work was performed to characterize the edge tone phenomenon. The dependence of the oscillating frequency on some of the main parameters of the configuration including the nozzle-wedge distance and the profile and the mean velocity of the jet was measured. The frequency was obtained from the FFT of a pressure sensor signal. The measured and computed frequencies are compared and show a very good agreement. For both the CFD simulations and the experiments the phase of the oscillation and the pressure at specified points on the wedge are correlated. For the experiments the phase of the flow oscillation is determined with the help of flow visualization pictures obtained by a high speed digital camera. From the CFD results spatial distribution of acoustical sources are also observed and correlated with pressure distribution on the wedge and with the phase of the oscillating flow. Some aspects of the initial transients are also presented. 
1pMUb4. Influence of the edge tone (mouth tone) on the sound of flue organ pipes as a function of pipe scaling. Judit Angster (Fraunhofer Institute for Building Physics, Nobelstrasse 12, 70569 Stuttgart, Germany, an@ibp.fhg.de), Andras Miklos (Steinbeis Transfer Center Applied Acoustics, Landauer Str. 24, D-70499 Stuttgart, Germany, Andreas.Miklos@urz.uni-heidelberg.de)

The sound quality of an organ pipe is mainly influenced by the attack transients. This onset is first dominated by the edge tone, while later the pipe resonator will play a more important role. To understand the physics of a flue organ pipe, it is necessary to analyse the edge tone, the acoustic properties of the pipe resonator, the attack transient and the stationary sound of the pipe. Several pipe ranks have been investigated in the anechoic room of the Fraunhofer IBP: Nachthorn, Diapason, Gamba, Octave, Flute, Geigenprinzipal, Salizional. By the evaluation all physical effects contributing to the production of sound were taken into account. In the present paper about the physical effect of the edge tone on the production of the pipe sound will be reported.

\title{
$5: 40$
}

1pMUb5. Development of an adjustable pipe-foot model of a labial organ pipe. Hubert Außerlechner (Fraunhofer Institute for Building Physics, Nobelstrasse 12, 70569 Stuttgart, Germany, hubert.ausserlechner@ibp.fraunhofer.de), Judit Angster (Fraunhofer Institute for Building Physics, Nobelstrasse 12, 70569 Stuttgart, Germany, an@ibp.fhg.de), Andras Miklos (Steinbeis Transfer Center Applied Acoustics, Landauer Str. 24, D-70499 Stuttgart, Germany, Andreas.Miklos@urz.uni-heidelberg.de)

In order to understand the physical processes involved in the build-up of the sound signal in a pipe (in our case a labial organ pipe) a pipe-foot model has been developed, with which the main important parameters, such as positions of the lower- and upper-labium, the wind pressure in the foot and the width of the flue can be adjusted. Moreover, different types of languids and pipe bodies (resonators) can be attached to the model. For the reason of corresponding to a real metal organ pipe these parts of the model are made of "organ metal." The reproducibility of measurements is provided by the micrometer screws applied for the adjustments. Flow and edge-tone measurements were carried out with the help of this model. A comparison with real organ pipes gives some indications for the range of the parameters. Because of the flexible adjustments and the large range of values of every parameter, it is possible to find different ranges with varying parameter settings for the occurrence of the edge-tone. The analysis of these measurement results shows the dependency of the edge-tone of the adjusted parameters.

\section{Session 1pMUc}

\section{Musical Acoustics and Psychological and Physiological Acoustics: Acoustics and Psychoacoustics of Pipe Organs}

\author{
Jonas Braasch, Cochair \\ Rensselaer Polytechnic Institute, Greene Bldg., 110 8th St., Troy, NY 12180, USA \\ David M. Howard, Cochair \\ University of York, Department of Electronics, Heslington, York, YO10 5DD, UK
}

\section{Invited Papers}

1pMUc1. Voicing documentation of a pipe organ. Christophe D'Alessandro (LIMSI-CNRS, B.P. 133, 91403 Orsay, France, cda @limsi.fr)

The paper begins with a brief presentation on the Parisian organ landscape, and then focuses on sound documentation of the historical organ of Sainte Elisabeth (Suret 1853, 3 keyboards and pedal, 40 stops, about 2500 pipes). Calibrated recordings of all the notes have been performed inside and outside the organ case, using several microphone positions. The methodology proposed by Pollard (J. Acoust. Soc. Am. 106(1), July 1999, 360-370) is extended and applied to sound analysis for all the notes of the instrument. The analogies between the proposed sound description (or "voicing quality," described in terms of sharpness, loudness, tristimulus coordinates, transient building, and spectral centre of gravity) and human voice quality description are discussed. Both voicing of individual pipes and voicing of the instrument are analysed. "Voicing of the instrument" means voicing variations of the pipes for different notes or registers of a same stop, for different stops of a same division, and for different divisions of the instrument. This methodology may be applied to documentation and characterisation of the "style" of the instrument in terms of its acoustic signature, provided additional information on recording conditions and room acoustics are available. 
1pMUc2. Documenting the acoustics of the Silbermann pipe organ at the Catholic Cathedral in Dresden before and after the restoration of 2002. Christian Ahrens (Musikwissenschaftliches Institut, Ruhr-Universität Bochum, Universitätsstr. 150, 44780 Bochum, Germany, christian.ahrens@rub.de), Jonas Braasch (Rensselaer Polytechnic Institute, Greene Bldg., 110 8th St., Troy, NY 12180, USA, braasj@rpi.edu), Sebastian Schmidt (Institute of Communication Acoustics, Ruhr-Universität Bochum, IC 1/142, Universitätsstr. 150, 44780 Bochum, Germany, sebastian.schmidt@ rub.de)

Gottfried Silbermann's pipe organ at the Catholic Cathedral of the Saxonian Court in Dresden was, with 47 stops, the largest instrument he had ever built. The instrument was evacuated during World War II and luckily survived intact, while the church itself was badly damaged. The instrument received a more romantic sound ideal after its resurrection in 1971. The biggest deviation from the original concept of Silbermann was the change from church pitch $\left(\mathrm{A}_{4}=415 \mathrm{~Hz}\right)$ to chamber pitch $\left(\mathrm{A}_{4}=440 \mathrm{~Hz}\right)$. In 2002, the instrument was renovated again with the goal to restore the instrument to the original design of 1755 . The work was commissioned to two local organ builders, Kristian Wegscheider and Jehmlich Orgelbau, and the authors received the unique opportunity to measure the instrument before and after the restoration. During the measurements, the pipes of each stop were recorded in the near field in steps of a third. The acoustical comparison between each pipe before and after the restoration shows significant differences that match the informal observations of expert listeners. In general, the pipes received more energy in the higher partials and had a better attack response. Another interesting finding is the improvement of the pipes' formants after the restoration.

\section{Contributed Papers}

\section{6:40}

1pMUc3. The physical characteristics of mechanical pipe organs and how much they allow the organist to influence the initial transient. Jose Grossinho (R. Francisco da Silva Marques, ${ }^{\circ} 5,2^{\circ}$ Dto, 2825-125 Caparica, Portugal, zefino@netcabo.pt)

It has been accepted by organists that one can change the timbre of the organ simply by varying the velocity of the key. Previous research (Nolle, 1941, 1992) shows clearly that there is a change in the initial transient of the sound with different pressure rise times (PRT) on the foot of the pipe. More recent research (Woolley, 2006) shows that in mechanical action organs there is no major difference in the velocity of the key when the organist thought there was. The velocity of opening of the pallet is one of the main aspects that influence the PRT. Calculations of the flexibility of mechanical parts from organs in Mafra, Portugal, were taken, as well as motion of the key, pressure on the pipe's foot and sound measurements on a model organ, using human touch on the key. Due to the pressure differences in the wind chest and the groove, it is difficult to control the pallet movement after it starts to open. The results show that there is a more efficient control of the velocity of opening of the pallet on more rigid actions, giving a better control to the organist over the timbre of the instrument.

\section{7:00}

1pMUc4. Very-low frequency range influence for free reed instruments physical modeling. Laurent P. Millot (IDEAT (University Paris 1), ENS Louis-Lumière, 7 allée du Promontoire, 93161 Noisy-le-grand, France, 1.millot@ens-louis-lumiere.fr)

Measurements of the inner and outer over-pressure for a diatonic harmonica were performed during blown, drawn, and bend notes notably. Using an energy analysis based on a nonregular spectral decomposition of the energy with only 10 frequency subbands, the IDS analysis, we have compared the relative weights of each subbands for both inner and outer pressure signals. This comparison underlines the key role played by the very low frequency range $(0-50 \mathrm{~Hz})$ and justifies a physical modeling without any waves occurrences but taking into account only acoustical flows descriptions. Within the presentation the audience will listen these phenomena and will access an explanation of the details of the physical modeling which has been used and which permits to refind chromatical playing on a diatonic harmonica, including the vocal tract player. We will also propose some clues to include a pipe in the modeling in order to study instruments like the sheng or the harmonium for instance. 


\title{
Session 1pNSa
}

\section{Noise, Physical Acoustics, and EURONOISE: Aeroacoustics I}

\author{
Philip J. Morris, Cochair \\ Penn State University, 233C Hammond Building, University Park, PA 16802, USA \\ Christophe Bailly, Cochair \\ Ecole Centrale de Lyon, 36, avenue Guy de Collongue, LMFA, Ecully, 69134, France
}

\section{Invited Papers}

1:00

\begin{abstract}
1pNSa1. Jet noise from large-scale turbulent structures. Philip J. Morris (Penn State University, 233C Hammond Building, University Park, PA 16802, USA, pjm@psu.edu)

The generation sound by large-scale turbulent structures in jets, where the structures are convecting supersonically with respect to the ambient speed of sound, is well understood. The pressure pattern generated by the large-scale structures couples directly with the radiated sound field. An instability wave model for the development of the large-scale structures gives excellent predictions of both the near and far acoustic fields. However, when the structures are convecting subsonically, there is no general agreement as to how or even whether they generate any noise directly. Recent experimental evidence has shown a remarkable similarity between the jet noise spectra in the vicinity of the peak noise radiation direction for both subsonic and supersonic jets. This suggests that the large-scale structures do contribute directly to jet noise radiation at all jet operating conditions. In the present paper a model is developed for the noise generation mechanism. Using experimental measurements of the far field noise, the near field pressure pattern required to generate the noise can then be calculated. The question of whether this pattern is consistent with a reasonable physical model for the evolution of the largescale turbulent structures is then discussed.
\end{abstract}

\section{$1: 20$}

1pNSa2. Effect of jet temperature on jet noise: data and requirements for modeling. Krishnamurthy Viswanathan (The Boeing Company, M/S 67-ML, P.O. Box 3707, Seattle, WA 98124, USA, k.viswanathan@boeing.com)

The modifications to the jet spectra caused by the heating of the jet have been the subject of experimental and theoretical research since the early 1970s. Early experimental data produced ambiguous and contradictory trends, which complicated the modeling efforts. Disparate theories have been proposed; however, no fundamental explanation or theory exists to date. First of all, a comprehensive experimental program has been carried out to establish the effect of temperature on jet noise. Detailed analyses indicate that in addition to the jet velocity $(\mathrm{Vj} / \mathrm{a})$, the jet temperature ratio, either stagnation or static, is an independent controlling parameter. The spectral shape at the lower radiation angles (measured from the jet inlet) is universal and independent of the jet Mach number and temperature. In the aft directions, the spectral shape is controlled by the jet temperature ratio, regardless of the jet velocity. These recently established trends have not been incorporated in any existing theory. The modeling of the effects of jet temperature and the suitability of the proposed formulations in existing theories are examined; the need for fresh thinking and the requirements for a good model are highlighted.

\section{Contributed Paper}

\section{$1: 40$}

1pNSa3. Correlations between the turbulent and the acoustic fields of a hot co-axial jet simulated by large-eddy simulation. Christophe Bogey (Ecole Centrale de Lyon, 36, avenue Guy de Collongue, LMFA, 69134 Ecully, France, christophe.bogey@ec-lyon.fr), Francois Coiffet (Ecole Centrale de Lyon, 36, avenue Guy de Collongue, LMFA, 69134 Ecully, France, francois.coiffet@ec-lyon.fr)

Correlations between the turbulent and the acoustic fields of a highspeed hot co-axial jet are calculated, in order to study noise generation mechanisms by a causality method. The jet flow was computed by a largeeddy simulation using specific aeroacoustic schemes. The simulation also provided directly the near-presure field, which was then extrapolated in the far field by solving the linear acoustic equations. The causality method is applied using flow quantities such as the velocity components, density, and temperature in the jet and the far field pressure, in the temporal as well as in the spectral domains. To exhibit and characterize the regions of noise generation, we are focusing on the correlation peaks, and in particular their location and corresponding frequencies. Moreover, there is an attempt to link the noise generation with the jet turbulence properties including intermittency and convection velocity. For instance, the turbulence at the end of the primary potential core was found to show high correlations with the far field pressure and significant intermittency, in a similar way as in single-stream jets. 


\section{Invited Papers}

2:00

1pNSa4. Simulation of jets exhausting from chevron nozzles and their noise. Ali Uzun (Florida State University, School of Computational Science, 400 Dirac Science Library, Tallahassee, FL 32306, USA, uzun@scs.fsu.edu)

Environmental concerns and strict noise regulations around major airports have made jet noise a crucial problem in present day aeroacoustics research, as it is the jet engine exhaust that is responsible for much of the noise generation during aircraft takeoff. Chevron nozzles have drawn a lot of attention recently due to their noise reduction benefits. Chevrons typically reduce low frequency noise at aft angles while providing an increase in high frequency noise at broadside angles relative to the jet. The streamwise vorticity generated by the chevrons affects the jet shear layer mixing and thus is responsible for the decrease or increase in noise over certain frequency ranges. The ultimate goal in chevron design is to decrease low frequency noise as much as possible while preventing the increase in high frequency noise. In our research, we are performing high-fidelity numerical simulations of jets exhausting from chevron nozzles to gain a better understanding of chevron jet shear layer mixing. These simulations employ a large eddy simulation tool utilizing state-of-the-art numerical techniques. In this talk, representative results from our research will be presented and an assessment of our current prediction capability of chevron jet noise will be provided.

2:20

1pNSa5. Reduced-order models for jet noise. Ann P. Dowling (University of Cambridge, Department of Engineering, Trumpington Street, CB2 1PZ Cambridge, UK, apd1@cam.ac.uk), Mohammed Afsar (University of Cambridge, Department of Engineering, Trumpington Street, CB2 1PZ Cambridge, UK, ma357@cam.ac.uk), Sergey Karabasov (University of Cambridge, Whittle Laboratory, Department of Engineering, Madingley Road, CB3 ODY Cambridge, UK, sak36@eng.cam.ac.uk), Tom P. Hynes (University of Cambridge, Whittle Laboratory, Department of Engineering, Madingley Road, CB3 ODY Cambridge, UK, tph@eng.cam.ac.uk)

The research reported here leads to a simple prediction methodology based on a reduced-order model for jet noise. The approach is a hybrid one made up of three components. Each component uses modeling and numerical techniques optimised to suit a particular purpose. The propagation of noise to the far field is captured via a new method for solution of the adjoint linearised Euler equations, describing how sound emitted by the jet is modified by propagation through the time-averaged but spatially varying jet flowfield. The directivity of the quadrupole sources is more general than is usually assumed and their statistical properties are modeled based on a RANS solution for the jet: the cross-correlation of the turbulent quadrupoles is modelled as Gaussian with length and time parameters proportional to the local length and timescales from the RANS solution, The constants of proportionality are determined through comparison with correlations from LES and from experimental data. Hence the source model is determined entirely from near field data and the far-field sound is then predicted with no empirical constants. Comparison between this predicted noise and experimental data is very good, across a wide spectral range and even at angles close to the jet axis.

\section{Contributed Paper}

2:40

1pNSa6. Numerical study of the influence of temperature and microjets on the radiated noise for a subsonic jet. Maxime Huet (ONERA, BP 72-29 avenue de la Division Leclerc, 92322 Châtillon, France, Maxime.Huet@onera.fr), Gilles Rahier (ONERA, BP 72 - 29 avenue de la Division Leclerc, $92322 \quad$ Châtillon, France, Gilles.Rahier@onera.fr), François Vuillot (ONERA, BP 72 - 29 avenue de la Division Leclerc, 92322 Châtillon, France, francois.vuillot@onera.fr)

Jet noise remains the principal nuisance source for an aircraft at take-off conditions. A previous work [1] showed that using micro-jets could be effective to modify the turbulence development and thus decrease the radiated pressure. This micro-jets effect is numerically studied for two jets. LES simulations of a cold $(M=0.9)$ and a hot $(M=0.636)$ jets, with and with- out micro-jets, are computed and the acoustic post-processing is performed using the Ffowcs-Williams and Hawkings surface formulation. The temperature effect on the noise is analyzed for the reference computations and, with micro-jets, the noise reduction is compared to measurements for a similar configuration [1]. Aerodynamic comparisons with experiments give a good comparison, excepted a too short potential core and overestimated turbulent kinetic energy. The noise decrease with the higher temperature and the modifications of the spectral densities are well captured by the simulations. The micro-jets provide a reduction up to more than $2 \mathrm{~dB}$ for the best angles. Turbulence modification is still under investigation and results will be presented. [1] Castelain, T., Béra, J., Sunyach, M., and Juvé, D., "Effect of microjets on a high-subsonic jet. Parametric study of far-field noise reduction,” 12th AIAA/CEAS Aeroacoustics Conference (2006).

\section{Invited Papers}

1pNSa7. On the separation of hydrodynamic and acoustic waves in linear free-shear flows. Anurag Agarwal (Institute of Sound and Vibration Research, University of Southampton, Highfield, SO17 1BJ Southampton, UK, aa2@isvr.soton.ac.uk), Gwenael Gabard (Institute of Sound and Vibration Research, University of Southampton, Highfield, SO17 1BJ Southampton, UK, gabard@soton.ac.uk)

The governing equations for sound propagation through free-shear flows, like jets and mixing layers, are the linearized Euler equations. These equations support both hydrodynamic and acoustic waves. For an aeroacoustician wishing to study the refraction effects of a sound source by shear flows, it is important to distinguish the acoustic solution from the hydrodynamic waves. Agarwal et al. (AIAA J., Vol. 42, No. 1, 2004) presented a technique to achieve this in the frequency domain. In this talk, we present a time-domain technique to separate the hydrodynamic and acoustic waves. The idea is to implement a filter that filters out only the acoustic wave solution from the linearized Euler equations. Some sample solutions are presented for two-dimensional free-shear flows and comparisons are made against known analytical solution for parallel flows and from solution obtained by approximate methods, which have a limited range of applicability. The advantage for the present technique is that it is applicable to arbitrary frequencies and flow profiles. 
1pNSa8. Turbulent flow noise around a zero-incidence airfoil. Olivier Marsden (Ecole Centrale de Lyon, 36, avenue Guy de Collongue, LMFA, 69134 Ecully, France, olivier.marsden@ec-lyon.fr), Christophe Bogey (Ecole Centrale de Lyon, 36, avenue Guy de Collongue, LMFA, 69134 Ecully, France, christophe.bogey@ec-lyon.fr), Christophe Bailly (Ecole Centrale de Lyon, 36, avenue Guy de Collongue, LMFA, 69134 Ecully, France, christophe.bailly@ec-lyon.fr)

A large eddy simulation (LES) of the flow around a NACA 0012 airfoil at zero incidence, at a chord-based Reynolds number of 500,000 and a Mach number of 0.22 , is presented. The aim is to show that high-order numerical schemes can successfully be used to perform direct acoustic computations of compressible transitional flow on curvilinear grids. At a Reynolds number of 500,000, the boundary layers around the airfoil transition from an initially laminar state to a turbulent state before reaching the trailing edge. Results obtained in the LES show a well-placed transition zone, and turbulence levels in the boundary layers in agreement with analytical developments as well as experimental data. Furthermore, the radiated acoustic field is determined directly by the LES, without the use of an acoustic analogy. Third-octave acoustic spectra are compared to experimental data, with deviations of around $3 \mathrm{~dB}$ per band exhibited.

\section{Contributed Paper}

\section{3:40}

1pNSa9. Preliminary screening of flow behavior around airfoils using a microphone phased array. Marcel C. Remillieux (Virginia Tech, Mechanical Engineering, 153 Durham 0238, Blacksburg, VA 24061, USA, mremilli@vt.edu), Ricardo A. Burdisso (Virginia Tech, Mechanical Engineering, 153 Durham 0238, Blacksburg, VA 24061, USA, rburdiss@vt.edu), William J. Devenport (Virginia Tech, Aerospace and Ocean Engineering, 224E Randolph Hall, Blacksburg, VA 24061, USA, devenport@vt.edu)

Recently, as a part of research projects for the National Renewable Energy Laboratory (NREL) and Sandia National Laboratories, several fullscale, wind-turbine airfoils were tested at various speeds (high Reynolds numbers) and angles of attack in the Virginia Tech Stability Wind Tunnel. This unique facility is aerodynamically closed and acoustically open, which allows better aerodynamic performance than a free jet facility, while maintaining comparable acoustic performance. Flow measurements consisted of monitoring the surface pressure around the airfoils with a distribution of pressure taps on the airfoil surface. Acoustic measurements were carried out with a microphone phased array. The phased-array data was post-processed to generate acoustic maps of the noise generated by the airfoils exposed to the flow as well as their noise spectra. In addition to the tests, twodimensional CFD computations of the flow field around the airfoils were carried out using a k- $\epsilon$ turbulent model. The acoustic maps clearly show the noise sources generated by potential turbulent flow around the airfoils. The very good correlation between the acoustic maps and the flow characteristics of the airfoils, both numerical and experimental, allows for phased-array measurements to be used as a tool for preliminary screening of the flow behavior around a given airfoil.

4:00-4:20 Break

Invited Paper

$4: 20$

1pNSa10. Experimental analysis of the wall aerodynamics and acoustic radiation of the trailing edge of an airfoil in subsonic flow. Cyrille Bonamy (Laboratoire d'Etudes Aérodynamiques (LEA), Université de Poitiers - ENSMA - CNRS, Bâtiment K, 40 Avenue du Recteur Pineau, 86022 Poitiers, France, cyrille.bonamy@lea.univ-poitiers.fr), Yves Gervais (Laboratoire d'Etudes Aérodynamiques (LEA), Université de Poitiers - ENSMA - CNRS, Bâtiment K, 40 Avenue du Recteur Pineau, 86022 Poitiers, France, yves.gervais@lea.univ-poitiers.fr)

This experimental study treats of the trailing edge noise mechanisms, especially broadband noise, resulting from the hydrodynamic wave diffraction on the sharp edge of a foil in flow, with the aim of improving its modeling. A detailed review of the dynamic behaviour of the phenomenon characteristic aerodynamic quantities (wall pressure and velocity fluctuations) has been made on a Naca 0012 airfoil and particular statistical data analysis have been performed (POD, temporal and spectral visualizations, spatial filtering by transducer array). Some models from the literature related to the wall pressure statistics (Corcos, Chase) have been adjusted and validated, and finally used for the modeling of the noise from the trailing edge of the airfoil. To conclude, measurements of the radiated acoustic pressure have been made in an anechoic wind tunnel, showing as a result the validity and limits of the implemented aeroacoustic model. The approach proposed by Chase in terms of wall pressure statistic modeling appears to be able to correctly represent the influence of the problem main parameters (Reynolds number, airfoil incidence).

\section{Contributed Papers}

\section{4:40}

1pNSa11. A numerical insight into the effect of confinement on trailing edge noise. Thomas Le Garrec (Arts et Métiers Paris Tech - Sinumef Lab, 151 bd de l'Hopital, 75013 Paris, France, thomas.le-garrec@paris.ensam.fr), Xavier Gloerfelt (Arts et Métiers Paris Tech - Sinumef Lab, 151 bd de l'Hopital, 75013 Paris, France, xavier.gloerfelt@paris.ensam.fr), Christophe Corre (LEGI Lab, BP 53, 38041 Grenoble Cedex 9, France, christophe.corre@hmg.inpg.fr)

The flow and the acoustic field around a 3D NACA 0018 airfoil at Reynolds 160000 with an angle of attack of $6^{\circ}$ are investigated numerically by direct noise computation to make comparisons with the experimental results of Nakano et al. (Experiments in Fluids, 2005) and the numerical simulations of Kim et al. (Heat and Fluid Flow, 2006). The direct noise computation of the flow around a 3D airfoil reaches the limits of current computational capacities. Indeed the main difficulty of such simulations comes from the large disparities between the fine scales of turbulence and the large wavelengthes of acoustic radiation which impose severe constraints on the meshes. In order to limit the number of points and to reduce the calculation cost, a multisize-mesh multitime-step strategy is adopted. The main purpose of the paper is to study numerically the influence of the confinement due to the top and bottom surfaces of the wind tunnel used in the experiments of Nakano et al. on the flow around the airfoil. The numerical results for a con- 
fined airfoil will be compared to the results obtained when the top and bottom walls are removed. So the noise can be directly linked to the vortices which develop in the wake of the airfoil.

\section{5:00}

1pNSa12. Coupled oscillations in the aeroacoustics of a Katana blade. Michel Roger (Ecole Centrale de Lyon, 36 Avenue Guy de Collongue, Centre Acoustique, 69134 Ecully, France, michel.roger@ec-lyon.fr)

The paper investigates experimentally the tonal characteristics of the aerodynamic sound of a Katana blade handled at high speed. Katana is the name of the Japanese sword used in martial arts. It is designed with a sharp cutting edge, a thick black-edge, and sometimes so-called blood-grooves. When handled through the air, the blade radiates sound at different frequencies. First a low-frequency, dominant sound is heard due to the von Karman vortex shedding in the wake of the blade. Second high-frequency tones are produced in the case of a Katana with grooves. The tones result from the self-sustained oscillations of the detached shear layers over the grooves which behave like cavities under a grazing flow. In the case of a Katana with no groove, other high-frequency tones are heard due to acoustic back-reaction on the Tollmien-Schlichting waves growing in the boundary layers. The variations of the acoustic signature with varying angle of attack and relative flow speed are analyzed in the paper, with emphasis on the coupling between the different mechanisms. The main trends are compared to simple self-sustained oscillation formulae. Finally the possible use of the sound as a criterion of inaccuracies in the handling of the Katana is discussed.

\section{$5: 20$}

1pNSa13. Large-eddy simulation of roughness-generated boundary-layer noise. Meng Wang (University of Notre Dame, Departmernt of Aerospace and Mechanical Engineering, Notre Dame, MD 46556, USA, m.wang@nd.edu), Qin Yang (University of Notre Dame, Departmernt of Aerospace and Mechanical Engineering, Notre Dame, MD 46556, USA, qyang@nd.edu)

A computational study is carried out to predict rough-wall boundary layer noise and elucidate noise generation mechanisms. As a first step, the sound radiation from a single hemispherical roughness element in a turbulent boundary layer at $R e_{\theta}=7500$ is investigated. The roughness height is $3.6 \%$ of the boundary layer thickness, or 95 wall units. The flow field is computed by large-eddy simulation. The velocity statistics show reasonable agreement with the experimental data measured at Virginia Tech. Acoustic calculations are performed based on the Curle-Powell integral solution to the Lighthill equation. The sound radiation is dominated by unsteady drag dipoles and their images in the wall, with the spanwise dipole of similar magnitude or stronger compared to the streamwise dipole. The viscous contribution to the drag dipole is negligible relative to the pressure contribution. Numerical experiments are performed to isolate the roles of vortex shedding and diffraction of convected hydrodynamic pressure by the roughness element; both are shown to be important noise source mechanisms. The effects of roughness height, upstream wake, and multiple roughness elements are discussed as well.

\section{$5: 40$}

1pNSa14. The mechanisms of roughness noise. William J. Devenport (Virginia Tech, Aerospace and Ocean Engineering, 224E Randolph Hall, Blacksburg, VA 24061, USA, devenport@vt.edu), Stewart A. Glegg (Florida Atlantic University, Department of Ocean Engineering, Boca Raton, VA 33431, USA, glegg@oe.fau.edu), Nathan Alexander (Virginia Tech, Aerospace and Ocean Engineering, 224E Randolph Hall, Blacksburg, VA 24061, USA, alexande@vt.edu), Ben Smith (Virginia Tech, Aerospace and Ocean Engineering, 224E Randolph Hall, Blacksburg, VA 24061, USA, besmith5@vt.edu)

The interaction of turbulence and a rough surface results in the radiation of sound that is not produced in smooth wall flows. The mechanisms by which this sound is generated are not well understood. Scattering of the convected turbulent pressure field by the surface irregularities seems to be a probable source when the roughness size is small compared to the boundary layer. The bluff body mechanisms of vortex shedding and unsteady drag from the roughness elements appear more likely to be dominant sources when those elements are large compared to the boundary layer. Demonstrating the existence of these mechanisms, their range of dominance, the extent to which they are distinct and can be characterized in terms of flow parameters that can be realistically modeled (such as the surface pressure spectrum), are all pre-requisites to the development of a robust prediction method. New insight into these issues has been obtained through measurements made with using a purpose built roughness noise facility in combination with fundamental theoretical analysis of the problem. The measurements include roughness noise spectra, wall pressure spectra and aerodynamics for a large variety of rough surfaces. This work is supported by the Office of Naval Research.

\section{Invited Paper}

\section{6:00}

1pNSa15. Modeling and prediction of fan noise. Edmane Envia (NASA Glenn Research Center, Acoustics Branch, M.S. 54-3, 21000 Brookpark Road, Cleveland, OH 44135, USA, edmane.envia-1@nasa.gov)

Fan noise is a significant contributor to the total noise signature of a modern high bypass ratio aircraft engine and, with the advent of ultrahigh bypass ratio engines like the geared turbofan, it is likely to remain so in the future. As such, accurate modeling and prediction of the basic characteristics of fan noise are necessary ingredients in designing quieter aircraft engines in order to ensure compliance with ever more stringent aviation noise regulations. In this paper, results from a comprehensive study aimed at establishing the utility of current tools for modeling and predicting fan noise will be summarized. It should be emphasized that these tools exemplify present state of the practice and embody what is currently used at NASA and industry for predicting fan noise. The ability of these tools to model and predict fan noise is assessed against a set of benchmark fan noise databases obtained for a range of representative fan cycles and operating conditions. Detailed comparisons between the predicted and measured narrowband spectral and directivity characteristics of fan noise will be presented in the full paper. General conclusions regarding the utility of current tools and recommendations for future improvements will also be given. 


\section{Contributed Papers}

6:20

1pNSa16. Contra-rotating fans noise prediction for jet engine performance optimization. Dax Au (ISAE, 10, av Edouard Belin, 31055 Toulouse, France, dax.au@isae.fr), Alexandre Leblanc (ISAE, 10, av Edouard Belin, 31055 Toulouse, France, alexandre.leblanc@isae.fr)

This work fits within the framework of the European research project VITAL, which is dedicated to the development of technologies aimed at reducing the environmental impact of jet engines. In this respect, a new concept of ducted contra-rotating fans is investigated. The present contribution to this project is to elaborate a noise prediction tool for performances optimization. On the basis of the helicoidal surface theory, Hanson previously developed a harmonic formulation for the propfan rotor/rotor interaction. This method is here extended in order to take into account the duct effects and the noise radiated by the rear struts. A computer program has been written to predict the pressure distribution in the near field and to estimate the noise spectra in the far field. The results are validated with computational fluid dynamics simulations and compared with available public data. Those predictions are then included in a global noise computation with the other engine acoustic sources (coaxial jet, combustor and so on), and a multidisciplinary optimizer is used for the engine noise minimization.

\section{6:40}

1pNSa17. Design of a low noise radial fan with computational aeroacoustics. Esra Sorgüven (Yeditepe University, Kayisdagi, 34755
Istanbul, Turkey, sorguven@yeditepe.edu.tr),Yilmaz Dogan(Arcelik A.S., Tuzla, 34950 Istanbul, Turkey, yilmaz.dogan@arcelik.com), Ergin Arslan (Arcelik A.S., Tuzla, 34950 Istanbul, Turkey, ergin.arslan@arcelik .com)

Aim of this study is to employ computational aeroacoustics methods in order to design a fan system. Design objectives of this study are to decrease the flow-induced noise, to provide the necessary flow rate and pressure increase. During the design, modern computational methods will be employed in addition to traditional turbomachinery design methods. The design procedure begins with a baseline fan system design, which is based on traditional semi-empirical correlations. Flow through this fan system is analyzed via computational fluid dynamics. Pressure fluctuations on rotating and stationary solid surfaces are determined to calculate sound sources of the system. The baseline design is improved by eliminating the areas where powerful sound sources occur. Flow analysis and sound prediction is repeated to improve fan system further. This step is repeated until the desired sound power level is achieved. This design procedure employs numerical analysis instead of experiments. Therefore it will be more effective and less time consuming than the experimental trial and error design procedures. The number of prototypes and necessary measurements are decreased dramatically.

\section{Invited Paper}

1pNSa18. Computational AeroAcoustics of Realistic Co-Axial Engines. Stephane Redonnet (ONERA (French Aerospace Center), CFD \& Aeroacoustics Department, BP 72, 29 avenue de la division Leclerc, 92322 Chatillon Cedex, France, stephane.redonnet@onera.fr), Ciprian D. Mincu (ONERA (French Aerospace Center), CFD \& Aeroacoustics Department, BP 72, 29 avenue de la division Leclerc, 92322 Chatillon Cedex, France, dmincu@onera.fr), Eric Manoha (ONERA (French Aerospace Center), CFD \& Aeroacoustics Department, BP 72, 29 avenue de la division Leclerc, 92322 Chatillon Cedex, France, eric.manoha@onera.fr), Yann Druon (Airbus S.A.S, Department of Acoustic \& Environment, 316 route de Bayonne, 31000 Toulouse, France, yann.druon@airbus.com), Bastien Caruelle (Airbus S.A.S, Department of Acoustic \& Environment, 316 route de Bayonne, 31000 Toulouse, France, bastien.caruelle@airbus.com)

This study, that is relevant from the turbofan engines noise prediction/reduction, aims at CAA-computing the aft fan noise propagation/radiation of a realistic full-3D exhaust (with pylon and internal bifurcations), the latter being affected of (i) typical in-flight (take-off) thermodynamic conditions and of (ii) a representative fan noise modal content. As for previous studies conducted over baseline geometries, this CAA computation is conducted following the usual hybrid process, where a preliminary aerodynamic calculation provides a heterogeneous steady mean flow on which an acoustic calculation is then conducted A RANS computation is first performed, delivering the stationary jet mean flow characterizing the 3D exhaust in its typical 'take-off flight' $(\mathrm{M} \infty=0.25)$. A CAA grid ( 22 blocks, 28 millions cells) is then derived from the CFD one, before the RANS steady jet mean-flow is interpolated on it. After what the CAA computation is computed, a fan noise mode $(26,1)$ being emitted at a reduced frequency of $\mathrm{kR}=30(1 \mathrm{BPF})$ in the upstream of the engine's secondary exhaust, and numerically propagated along and outside the latter. Finally, a Kirchhoff post-treatment provides the far-field radiation characterizing these engine geometry, modal content and thermodynamic conditions. 


\title{
Session 1pNSb
}

\section{Noise and EURONOISE: Action Planning and Global Solutions for Urban Noise I}

\author{
Klaus Genuit, Cochair \\ HEAD acoustics GmbH, Ebertstrasse 30a, Herzogenrath, 52134, Germany \\ Itziar Aspuru Soloaga, Cochair \\ Labein-Tecnalia, C/Geldo-Parque Tecnologico de Bizkaia, Derio, 48160, Spain
}

\section{Invited Papers}

\author{
1:00
}

1pNSb1. Strategies for noise action plans. Paul De Vos (DHV BV, Postbus 1132, NL 3800 BC Amersfoort, Netherlands, paul .devos@dhv.com)

Following the requirements of the Environmental Noise Directive, Noise Action Plans should have been produced. The plans are supposed to build on two important pillars: the strategic noise maps and the consultation with the local residents. In some cases, the plan is considered as a mere statement of the long term policy of the authority under concern. In other cases, it is set up as an implementation plan with clear and measurable targets. Also the focus varies: only the highest noise levels could be attacked or the large numbers of exposed citizens, or even the overall annoyance. Target noise levels may lead to confusion both for local decision makers and citizens. Also, the preservation and possible creation of quiet areas can be envisaged. The use of an equal annoyance indicator helps to set the targets in the right perspective. Translation into different levels of quality of life is recommended. In terms of cost and benefits, the options for city councils are very limited. Due to a lack of European legislative power, cities tend to set their own rules, e.g. for road vehicles. Several initiatives to propose toolkits for action plans, comparable to the best practice guide for noise mapping, are welcomed but require harmonization and dissemination.

\section{$1: 20$}

1pNSb2. From strategic noise maps to action plan: Perspective of Spanish main roads. Maria Dolores Jimenez (Dirección General de Carreteras del Ministerio de Fomento, Paseo de la Castellana 67, 28071 Madrid, Spain, mdjimenez@fomento.es), Jesus Rubio (Dirección General de Carreteras del Ministerio de Fomento, Paseo de la Castellana 67, 28071 Madrid, Spain, jjrubio@fomento.es), Fernando Segues (CEDEX Centro de Estudios de Técnicas Aplicadas, Alfonso XII, 3-5, 28014 Madrid, Spain, fsegues@cedex.es), Pilar Fernandez (Labein Tecnalia, Parque Tecnologico Bizkaia, 48160 Derio, Spain, pfernandez@labein.es)

The Direccion General de Carreteras of the Spanish Ministry of Transport is finishing the process of Strategic Noise Mapping of Spanish major roads (EGRA). The whole National road network that has been noise mapped for the first END has a length of $6.400 \mathrm{~km}$ round. The present paper contains - Some conclusions from the process of noise mapping; - A general view of the process of making decisions about Action Plans; - Definition of the first steps to decide the scope of Action Plans: design of a pilot study to establish the elements needed technical studies, coordination with other administration bodies and public involvement.

\section{$1: 40$}

1pNSb3. Decision support system for Action Planning in the framework of the European Noise Directive. Jeroen Borst (TNO Science and Industry, Stieljesweg 1, 2628CK Delft, Netherlands, jeroen.borst@tno.nl), Walter J. Lohman (TNO, P.O. Box 49, NL2600 AA Delft, Netherlands, walter.lohman@tno.nl), Arno R. Eisses (TNO, Stieltjesweg 1, P.O. Box 155, 2600 AD Delft, Netherlands, Arno.Eisses@tno.nl), Henk M. Miedema (TNO, P.O. Box 49, NL-2600 AA Delft, Netherlands, henk.miedema@tno.nl)

The European Noise Directive (END) requires assessment of noise exposures as well as the formulation of Action Plans for the reduction of the number of people harmfully affected by environmental noise. TNO is developing a decision support system for noise mitigating measures. The proof of concept of such a system for road noise is presented here. On the basis of a detailed noise map, for each road segment an indication is given for the amount of negative effect (e.g., number of people being highly annoyed) per meter it is causing. On the basis of the characteristics of the road segment, the system suggests possible noise mitigation measures. The effect of the measure chosen by the user, such as the application of silent road surface types or lowering speed limits, can be interactively explored with the system. It directly shows the updated detailed noise contour maps as well as indicators describing the impact after a measure has been applied though the interactive interface. 
1pNSb4. Urban Noise Action Planning - an integrated approach towards noise abatement in the frame of urban development: Tools and recommendations developed within the SILENCE project. Melanie Kloth (Polis, rue du Trône 98, 1050 Brussels, Belgium, mkloth@polis-online.org)

Noise action planning following the European Directive on Environmental Noise is for many cities a new, additional task. It will probably be not very successful when considered as mere duty separated from other urban policy areas. Today, noise does not rank high on the local political agenda. Human and financial resources provided for noise abatement tend to be low. Furthermore, looking at concrete noise abatement measures it becomes clear that most effective measures also impact on other policy areas (air quality, mobility, etc.). Therefore it is advisable to link noise action planning closely with other urban plans like development plans, mobility plans, clean air programmes, etc. This requires a strong involvement of the relevant stakeholders as well as knowledge about the potential impact of noise abatement measures on other policy areas. To support local authorities in this complex task the SILENCE project developed a guidebook which comprises recommendations on organisational settings, on the involvement of stakeholders and the public, the action planning process as well as on a range of noise abatement measures. The description of these measures refers not only to their noise abatement potential but takes also into account their - positive or negative - impact on other policy areas.

2:20

1pNSb5. Noise Action Plan in the Ile-de-France region: a complex process. Fanny Mietlicki (Bruitparif, 25 rue Coquillière, 75001 Paris, France, fanny.mietlicki@bruitparif.fr)

The transposition of the European Noise Directive (END) into French law was achieved in April 2006. Noise maps have to be established before June 30, 2007 and action plans have to be set up before July 18, 2008 for agglomerations with more than 250,000 inhabitants and for major infrastructures. The State representatives at the departmental scale are in charge of the infrastructure maps, while the local authorities are in charge of the agglomeration maps. The Paris agglomeration which counts 11 million inhabitants is made of 396 towns. Since some of them are grouped into cooperation structures for noise issues, there are 238 entities in charge of the application of the END in the Paris agglomeration. To face this administrative complex situation, Bruitparif has developed a program to help local authorities in setting up the END. Different actions are lead: - providing information and technical assistance on how to establish noise maps and action plans, - conducting noise measurements in order to validate maps and to provide complementary information, - coordinating actions and initiatives, - consolidating the map of the Paris agglomeration from the local maps in order to estimate the noise exposure, - preparing guidelines for the action plans.

\section{2:40}

1pNSb6. Action planning procedures and realized action plans of municipalities and cities - results from the implementation of END. Markus Petz (ACCON GmbH, Ingenieurbüro für Schall- und Schwingungstechnik, Gewerbering 5, 86926 Greifenberg, Germany, markus.petz@accon.de)

For many federal states of Germany the Strategic Noise Maps were calculated and published in 2007. For some cities it will be shown, how a standard procedure for preparation of a noise action plan was defined together with responsible authorities from the municipality. The chosen procedure also considered the deadline for finalisation and the commencement of an action plan at July, 2008. Based on Strategic Noise Maps and hot spot analyses a catalogue of measures for noise reduction within a community was investigated and evaluated (cost/effectiveness). Together with representatives of traffic-, town- and environmental planning departures of the municipalities efficient measures were discussed and selected considering other local planning interests, financial possibilities and public acceptance. The draft action plan were published and public demurs have been weighted. In July, 2008 the action plans came into force.

\section{Contributed Papers}

3:00

1pNSb7. Action plans (PPBE) : experience of municipal noise fighting plans. Eric Gaucher (Acoustique \& Conseil, 17-19 rue des Grandes Terres, 92508 Rueil-Malmaison, France, eg@acoustique-conseil.com)

The European Noise Directive (END) relating to the assessment and management of environmental noise requires the elaboration of action plans (Plans de Prévention du Bruit dans l'Environnement PPBE). However, these plans are limited to their concerned sources: trains, roads, planes and industrial plants. Municipal noise fighting plans (Plans Municipaux de Lutte contre le Bruit PMLB) taking equally into account any annoying sound situation, regardless of its nature, have been developed and implemented in around forty towns in France, with increasing success before the END was published. Acoustique \& Conseil have already implemented thirty PLMBs. This experience has lead us to think that the strict application of PPBEs will in most cases not answer all the expectations of the concerned populations, in terms of solutions to their daily noise problems. It therefore seemed relevant for us to present a synthesis of the situations encountered during our works in order to enrich the approach of towns willing to enlarge their actions beyond those of the END. Now that it is time to elaborate the PPBE, it seems relevant and appropriate to implement PLMBs equally for the towns concerned.
$3: 20$

1pNSb8. Urban and building acoustics management in the next decades: a matter of prevention, simplification and education. João G. Baring (Univ. of São Paulo - Facty. of Archt. and Urban., Rua Rafael Clark 85, 05526 - 010 São Paulo, Brazil, jbaring@uol.com.br)

In Brazil, in cities like São Paulo and Rio de Janeiro, noise pollution control has been carried out mainly in the form of repression. In the seventies and eighties, people joined together to fight against noisy government projects, and government fought noisy industries and commercial establishments. Since then, people have become aware of their rights, but have learned little about their own obligations on the matter. The author researched this process in order to find a better way for the future and concluded that prevention must be enforced through noise codes, standards must include easier survey methods, and low cost devices for noise control must be available. Most important, education must be improved in all levels, to make every citizen a partner of noise control at home, at work and elsewhere in the cities. This paper points out some ways of dealing with this process.

\section{$3: 40$}

1pNSb9. NERS-analysis extended to include the existence of neighbouring quiet areas. Åsa Stenman (Acoustic Control AB, Tumstocksvägen 1, SE-187 66 Täby, Sweden, stenman@acoustic.se), Peter 
Malm(Acoustic Control AB, Tumstocksvägen 1, SE-187 66 Täby, Sweden, peter.malm@acoustic.se), Magnus Lindqvist (Stockholm Environmental \& Health Administation, Box 38024, SE-100 64 Stockholm, Sweden, magnus .lindqvist@miljo.stockholm.se)

One of the goals for the EC financed Integrated Project Quiet City Transport (QCITY), is to provide European city administrations with validated analysis tools and technical noise control solutions for the efficient production of noise action plans. A part of the QCITY project work is to produce complete noise maps and hot-spot analysis for a part of Stockholm. This paper will focus on the Noise Environmental Rating System (NERS), a tool for performing hot-spot analysis on noise maps, developed within the QCITY project. The input parameters that have been used in previous studies [Internoise-07_411, Å. Stenman, P.-Malm, 2007] are outdoor noise on all floors, number of inhabitants per building and the facade sound insulation index. Previous studies, such as the "Stockholm score rating model (Trafikbuller och planering II, Stockholm, 2004) show a strong correlation between noise annoyance and the existence of quiet nearby areas. The NERS-analysis in this work has therefore been extended to include quiet areas.

\section{4:00-4:20 Break}

\section{$4: 20$}

1pNSb10. NERS-analysis extended to include noise levels measured on city courtyards. Peter Malm (Acoustic Control AB, Tumstocksvägen 1, SE-18766 Täby, Sweden, peter.malm@acoustic.se), Åsa Stenman (Acoustic Control AB, Tumstocksvägen 1, SE-187 66 Täby, Sweden, stenman@acoustic.se), Thomas Hammarlund (Environment Administration, Karl Johansgatan 23, SE-414 59 Göteborg, Sweden, thomas .hammarlund@miljo.goteborg.se)

One of the goals for the EC financed Integrated Project Quiet City Transport (QCITY), is to provide European city administrations with validated analysis tools and technical noise control solutions for the efficient production of noise action plans. A part of the QCITY project work is to produce complete noise maps and hot-spot analysis for the entire Gothenburg area. Earlier studies have shown that calculated noise levels from traffic at inner city courtyard are lower compared to actual measurements. Gothenburg city have performed over 700 measurements on city courtyards. The measurements are included in the NERS-analysis to show the influence of the actual courtyard noise levels. The study covers a smaller area in the central part of Gothenburg where the measurements have been made.

\section{Invited Papers}

\section{4:40}

1pNSb11. Auralisation and psychoacoustic evaluation of traffic noise scenarios. Sandro Guidati (HEAD acoustics GmbH, Ebertstrasse 30a, 52134 Herzogenrath, Germany, claudia.erens@head-acousitcs.de)

The evaluation of vehicle noise is mostly done using the A-weighted sound pressure level, e.g., the Lmax for pass-by noise or the Leq for community noise. Unfortunately, the $\mathrm{dB}(\mathrm{A})$-value does not reflect the annoyance effect of vehicle noise correctly. During the first phase of the European Research Project QCity a metric for psychoacoustic evaluation of single pass-by events has been developed. In the next step this metric is adapted for the evaluation of complex traffic scenarios calculated by traffic flow simulations. Psychoacoustic evaluation requires time signals. Single values or third octave spectra are not sufficient to investigate all relevant effects (e.g., annoying time patterns, masking). The time signals are calculated combining advanced resynthesis with exterior noise simulation techniques. Hereby, it is possible to investigate the effect of e.g., low noise surfaces or various traffic control measures (traffic light vs. roundabout). Since a standard traffic scenario includes a very large number of single pass-by events special focus lies on fast processing techniques. This paper gives an overview of the developed algorithms and presents first results of the psychoacoustic evaluation of traffic scenarios.

1pNSb12. Lions Bay noise mitigation program. Duane E. Marriner (Wakefield Acoustics Ltd., 301-2250 Oak Bay Avenue, Victoria, BC V8R 1G5, Canada, duane@wakefieldacoustics.com)

The Ministry of Transportation (MoT) of the Province of B.C. noise abatement policy requires that environmental community noise impacts of highways projects involving new or substantially upgraded highways be assessed and mitigation implemented where warranted. This paper presents an extraordinary noise model developed to mitigate residential areas of the Village of Lions Bay on the rugged B.C. coastline from the Sea-to-Sky Highway connecting Vancouver, B.C. to Whistler, B.C. home of the Winter Olympic and Para-Olympic Games in 2010. The Cadna/A Version 3.9.15 software was used to develop a new 4 lane split grade alignment model incorporating two mini-interchanges. With the objective of achieving a $10 \mathrm{dBA}$ noise reduction benefit, mitigation in the form of quiet pavement (OGA), traffic calming and 5 meter high absorptive sound walls or noise barriers were introduced into the model which included 150 mountain side residential receptors along the $2 \mathrm{~km}$ long corridor at elevations up to 35 meters above the project. Noise reduction benefits for dwellings were further analyzed to determine contributions during each stage of project development by creating a super user, modular, multi-layer noise model of Lions Bay. This work was carried out under the sponsorship of BCMoT.

\section{$5: 20$}

1pNSb13. Tire/road noise reduction. Alexander Ossipov (Goodyear, Ave G. Smith, L-7750 Colmar - Berg, Luxembourg, alexander_ossipov@goodyear.com), Marc Engel (Goodyear, Ave G. Smith, L-7750 Colmar - Berg, Luxembourg, marc_engel @ goodyear.com)

Traffic running on high speed roads is a considerable source of noise annoyance and is frequently a source of complaint by the general public. One of the components of this noise is generated by the interaction between motor vehicles tires and the road surface. The amplitude and frequency content of this noise is a function of many parameters, including the road surface texture, tire dimensions, tire materials, and construction and the tread pattern design. The paper provides detailed insight concerning the factors influencing exterior (environmental) and interior (vehicle) tire/road noise reduction and includes recommendations with regard to optimize tire design. 


\section{Contributed Papers}

\section{$5: 40$}

1pNSb14. Calculations of low height noise barriers efficiency by using Boundary Element Method and optimisation algorithms. Marine Baulac (CSTB, 24 rue Joseph Fourier, 38400 Saint-Martin-d'Hères, France, marine.baulac@cstb.fr), Arnaud Guillou (Institut National de Métrologie (LNE-INM/Cnam), 61 rue du Landy, 93210 La Plaine Saint Denis, France, guillou.arnaud@gmail.com), Jérôme Defrance (CSTB, 24 rue Joseph Fourier, $\quad 38400 \quad$ Saint-Martin-d'Hères, France, jerome.defrance@cstb.fr), Philippe A. Jean (CSTB, 24 rue Joseph Fourier, 38400 Saint-Martin-d'Hères, France, philippe.jean@cstb.fr)

Nowadays, roads and railways are often quoted as the most annoying source of noise for the living environment. This problem of ground transportation noise is present in extra-urban environments as well as in town centres. Traditional noise barriers are in many cases not suited for acoustic protection in urban areas mainly because of their height. Previous studies have shown a significant efficiency of low height noise protections (kind of urban furniture) for the decreasing of noise levels in semi-opened areas such as city parks, places, and large avenues. However, the simulations of low height noise protections requires specific numerical codes since ray tracing methods are not suited for barriers lower than around $2 \mathrm{~m}$. This paper proposes a way to implement an approach with allow to simulate low height noise protections in engineering calculation methods. This can be done by creating a database using a Boundary Element Method numerical code combined with optimisation algorithms. Work is still in progress since only $2 \mathrm{D}$ configurations are considered at the moment.

\section{6:00}

1pNSb15. Status of noise abatement measures for roads in Switzerland. Laurent Cosandey (Office Fédéral de l'Environnement, OFEV, 3003 Berne, Switzerland, laurent.cosandey@bafu.admin.ch), Kirk Ingold (Office Fédéral de l'Environnement, OFEV, 3003 Berne, Switzerland, kirk.ingold@bafu.admin.ch), Tommaso Meloni (Office Fédéral de l'Environnement, OFEV, $3003 \quad$ Berne, Switzerland, tommaso.meloni@bafu.admin.ch), Nicole Luethi (Grolimund und Partner AG, Entfelderstr. 41, 5000 Aarau, Switzerland, Nicole.Luethi@gundp.ch), Hansueli Pestalozzi (Grolimund und Partner AG, Entfelderstr. 41, 5000 Aarau, Switzerland, hansueli.pestalozzi@gundp .ch)

In 2006, a comprehensive survey on the status of noise abatement measures for all roads was carried out in Switzerland for the first time. This survey recorded the costs of remedial work and noise protection measures completed to date (approx. CHF 1 billion) or still outstanding (approx. CHF 3 billion). An account is also given of the distribution among different types of measures, and of the protective effects on the public. As well as indicating the financial resources required for remediation of the Swiss road network on schedule, the survey highlights measures for controlling noise emissions at source which need to be implemented urgently.

\section{6:20}

1pNSb16. Influence of noise source representation on the estimation of specific descriptors close to traffic signals. Arnaud Can (LICIT, ENTPE/INRETS - Université de Lyon, rue Maurice Audin, 69518 Vaulx-enVelin Cedex, France, can@entpe.fr), Ludovic Leclercq (LICIT, ENTPE/INRETS - Université de Lyon, rue Maurice Audin, 69518 Vaulx-enVelin Cedex, France, leclercq@entpe.fr), Joel Lelong (INRETS, 25 av. F. Mitterrand, case 24, 69675 Bron, France, lelong@inrets.fr)

Considering traffic dynamics greatly improves noise estimation in urban area. This can be achieved by coupling a dynamic traffic model with both emission laws and sound propagation calculation. This paper focuses on the influence of noise source representations in the combination with the traffic model. Several representations are tested: point sources and homogeneous line sources of different sizes. We aim at evaluating how these representations correctly estimate classical descriptors (LAeq and statistical descriptors) and specific descriptors able to capture noise dynamics at a traffic signal scale. Three typical urban situations are studied: in front of, upstream and downstream a traffic signal. Noise source representation can be coarse for classical descriptors calculation if traffic dynamics is precisely described. On the contrary, noise source representation should be refined to precisely assess noise dynamics.

6:40

1pNSb17. Evaluation of Directive 2000/14/EC on outdoor machinery noise. Michael G. Dittrich (TNO Science and Industry, PO Box 155, 2600 AD Delft, Netherlands, michael.dittrich@tno.nl)

The European Directive 2000/14/EC sets a requirement for noise labeling for the sound power level of 57 types of outdoor equipment, and sound power limits for 22 of these. In the NOMEVAL project the Directive and its amendment 2005/88/EC were evaluated, resulting in recommendations for an update of the equipment list, the noise limits and the test codes. A European database of noise emission data was assessed, and environmental bodies and industry were consulted. The recommendations were also based on environmental, technical and economic impact assessments. A new environmental indicator was applied to rank the impact of the different equipment types. It was found that many types currently without noise limits have a higher impact than those with noise limits. Some new types of equipment have been identified including snowmobiles, mobile waste breakers and screens, motorised brooms, handheld cut-off saws, and power pruners. The technical impact assessment was based on current technology and trends and the feasibility of new or stricter limits. The economic impact assessment was based on estimated societal benefits versus the estimated additional cost to the consumer or purchaser. Finally, a number of instruments for further reduction of outdoor equipment noise were proposed.

\section{7:00}

1pNSb18. Some algebra and statistics on isolated noise events. Michel Maurin (INRETS-LTE, case 24, 69675 Bron cedex, 69500 Bron, France, maurin@inrets.fr)

Noise indices related to isolated emerging noise events are calculated on variable finite durations $\Delta \mathrm{T}_{\mathrm{ev}}$, as opposed to instantaneous levels or indices on constant conventional periods $\Delta \mathrm{T}(1 \mathrm{~h}, 24 \mathrm{~h}, \ldots)$. Here they are defined by TEL (transit exposure levels) or Leq $\mathrm{L}_{\Delta \mathrm{Tev}}$ on every $\Delta \mathrm{T}_{\mathrm{ev}}$. There are classical formulas to pass from many $\mathrm{TEL}_{\mathrm{i}}$ to the Leq on a longer period including many events. When $\mathrm{TEL}_{\mathrm{i}}$ are supposed equal to TEL one gets classicaly $\mathrm{Leq}_{\Delta \mathrm{T}}=\mathrm{TEL}+10 \log \mathrm{n}+\mathrm{K},(\mathrm{CNR}, \mathrm{NEF}$ for instance). In algebra, the approach develops some algebraic relations and conditions between total duration $\Delta \mathrm{T}$, the global part of all $\Delta \mathrm{T}_{\mathrm{ev}, \mathrm{i}}$ and their number $\mathrm{n}$, in a way that TEL is clearly emerging from noise level without the isolated events. It is a manner to find a general result like "events kill events," say that too many events render not emerging any new one. One finds the same with a statistical approach and other appropriate technics (quantiles for instance). And coming on with probabilistic tools, the Bêta distribution renders possible some characterisation of the graphical look of the time evolution of noise level during $\Delta \mathrm{T}_{\mathrm{ev}}$. This work has partly been done with $\mathrm{V}$. Catto of Lyon 1 University. 


\title{
Session 1pNSc
}

\section{Noise and EURONOISE: Noise Mapping Techniques and Uncertainties I}

\author{
Richard Peppin, Cochair \\ Scantek, Inc., 7060 Oakland Mills Rd., Columbia, MD 20146, USA \\ Wolfgang Probst, Cochair \\ DataKustik GmbH, Gewerbering 5, Greifenberg, 86926, Germany
}

\section{Contributed Paper}

1:00

1pNSc1. Optimizing localization of noise monitoring stations for the purpose of inverse engineering applications. Malgorzata Reiter (Gdansk University of Technology, Multimedia Systems Department, 11/12 Gabriela Narutowicza Street, 80-952 Gdansk, Poland, reiter.m@sound.eti.pg.gda.pl), Jozef Kotus (Gdansk University of Technology, Multimedia Systems Department, 11/12 Gabriela Narutowicza Street, 80-952 Gdansk, Poland, joseph@sound.eti.pg.gda.pl), Andrzej Czyzewski (Gdansk University of Technology, Multimedia Systems Department, 11/12 Gabriela Narutowicza Street, 80-952 Gdansk, Poland, andcz@ sound.eti.pg.gda.pl)

Long-term environmental monitoring of noise levels can be done using autonomous measurement stations. Because of the high cost of monitoring systems and management of these stations, it is essential to identify how many of measuring localization points are really required. In cases related to complex noise generation schemes, when there are various noise sources, the differences between calculations and measurements can be difficult to estimate. Therefore, it is vital to find some most appropriate locations for measurement stations which would ensure obtaining an adequate number of measurement results to be employed in the reverse engineering. These measurements can be then utilized to update dynamic noise maps. Furthermore, predictive noise models may be developed accordingly to certain local requirements. This could result in a better accuracy of dynamic noise maps. The paper focuses on defining the proper choice of the measurement points localizations. The experiments described include a comparison between real-life measurement results performed with the Multimedia Noise Monitoring System developed at the Multimedia Systems Department of the Gdansk University of Technology and the noise level prediction results. The optimization of the number and location of noise monitoring points with regard to the measurement accuracy is also discussed.

\section{Invited Paper}

\section{$1: 20$}

1pNSc2. Acoustical design margins: Uncertainty in prediction and measurement of community noise. Robert Putnam (Siemens Power Generation, 4400 Alafaya Trail, Orlando, FL 32826, USA, bob.putnam@ @iemens.com), Roland Hetzel (Siemens Power Generation, Beethovenring 8a, 91090 Effeltrich, Germany, roland.hetzel@siemens.com)

Compliance with regulatory requirements for sound levels in communities adjacent to industrial or power generating facilities is typically a contractual commitment, with the potential for significant financial penalties in the event of noncompliance. Uncertainties at any stage of the design, specification or prediction of plant sound level may be accounted for as part of the overall plant acoustical design margins. There are also additional uncertainties in terms of compliance sound measurement surveys, that are commonly referred to as either "test tolerance," or "instrumentation tolerance and measurement uncertainty." From the viewpoint of the plant equipment supplier all of the uncertainties associated with equipment design and specification are simply additive to all of the uncertainties of compliance measurements, since they all contribute to, or affect the selection of, the overall plant acoustical design margin. The discussion will address the types of uncertainties in source sound power levels and measurement error, tolerances, and confidence limits of field sound surveys, highlighting some seldom-treated aspects of uncertainty. Measurement uncertainties and the applicable combinatorial rules will be treated.

\section{Contributed Papers}

\section{$1: 40$}

1pNSc3. Possibilities of supporting municipal authorities in processes of acoustical environment management. Jan Ka Mierczak (Silesian University of Technology, Faculty of Organization and Management, ul. Roosevelta 26/28, 41-800 Zabrze, Poland, Jan.Kazmierczak@polsl.pl)

EU directives and state law acts oblige municipal authorities of European towns to create and exploit strategic acoustic maps. The further decisions of these authorities, considering both sustainable local development strategies and results of analysis of environmental effects (such as noise), ought to form suitable living conditions in the towns. The people, responsible for such decisions, ought to dispose suitable knowledge and skills. Author of this paper leaded in recent years the consortium of R\&D units involved in the research project, focussed on preparing technology of creating acoustic maps of urban areas. The project resulted in the method of acoustic mapping, based on GIS technology. Apart of "purely acoustical" problems, the forming of competencies of these members of municipal staff, responsible on preparation and then exploitation of acoustic maps, has been mentioned as a problem of essential importance in this research. In result of discussions with potential users of strategic acoustic maps, the need was formulated to give municipal units some effective tools of supporting them by means of offering necessary knowledge and expertises in the field of management of acoustical environment in urban areas. The concept of network-based system focussed on such a target is presented in the paper.

\section{2:00}

1pNSc4. Evaluation models for the noise diminution due to the phonique barrier walls. Mariana Arghir (Technical University of ClujNapoca, Faculty of Machines Design; Department of Mechanics and Computer Programming, B-dul Muncii; Nr. 103-105, 400641 Cluj-Napoca, 
Romania, marianaarghir@yahoo.com),Dan Borza(National Institute of Applied Sciences of Rouen, INSA Rouen, LMR, Ave l'Universite, BP8 76800 Rouen, France, dan.borza@insa-rouen.fr), Florin Blaga (University of Oradea, Str. Universitatii nr.1, 400641 Cluj-Napoca, Romania, florin_blaga2000@yahoo.com), Tiberiu Vesseleny (University of Oradea, Str. Universitatii nr.1, 400641 Cluj-Napoca, Romania, vasitata@yahoo.com), Geamilia Solea (Technical University of ClujNapoca, Faculty of Machines Design; Department of Mechanics and Computer Programming, B-dul Muncii; Nr. 103-105, 400641 Cluj-Napoca, Romania, marylandprod@gmail.com), Mariana Runcan (National Institute of Applied Sciences of Rouen, INSA Rouen, LMR, Ave l'Universite, BP8 76800 Rouen, France, mariruncan@yahoo.com)

The present paper contains our study regarding the diminution of the urban noise using the phonique barrier walls conformed to the EMPARA procedure, which is based on the simplified of the standard method for the measurements and computes of the surface traffic inside the urban agglomeration, as they are given in SRM2 laws (Netherlands standards). There are made the studies taking into account all the parameters that characterize the the noise and they are graphical representations having the "n-1" constant parameters and only one having the time variation law. In this way we are obtained the influence of each parameter in the diminution of the urban noise using the phonique barrier walls. In addition we realized a measurement for a real barrier wall inside the Oradea town. The comparison between them was enough good, that means our theoretical study is correct and characterizes the noise pollution and its diminution on the urban agglomeration. The measurement device was a CENTER 322 Sound Lever
Meter. This study contains the two steps for the "noise map" asked for the Romania integration in the European Union, and was made by the researchers of the Oradea University under the direction of the Prof. Dr. Ing. Mariana ARGHIR from the Technical University of Cluj-Napoca.

\section{2:20}

1pNSc5. Environmental impact of noise from mobile outdoor equipment. Foort De Roo (TNO Science and Industry, Stieljesweg 1, 2628CK Delft, Netherlands, foort.deroo@tno.nl), Eddy Gerretsen (TNO Science and Industry, Stieljesweg 1, 2628CK Delft, Netherlands, eddy.gerretsen@tno.nl), Jeroen Borst (TNO Science and Industry, Stieljesweg 1, 2628CK Delft, Netherlands, jeroen.borst@tno.nl)

As part of an evaluation study of European Directive 2000/14/EC relating to the noise emission by equipment for use outdoors, an environmental impact assessment was required to investigate the need for new or tighter noise limits. In contrast to other environmental noise sources, the operating location of outdoor machines is not fixed. As conventional environmental noise mapping could not be used for this purpose, an environmental indicator was developed. This indicator takes the following factors into account: numbers of equipment in use in the EU, typical average sound power level, tonal/impulsive/intermittent sound content, annual duration of operation, day/night operation and typical areas of use. The numbers of people affected were assessed by means of a series of noise maps, resulting in distribution curves characteristic for different types of environment (urban, suburban, rural, and others). The newly developed environmental indicator is a relatively simple quantity taking all relevant factors into account including annoyance.

\section{Invited Papers}

1pNSc6. Procedures of large scale noise mapping - examples based on German projects. Markus Petz (ACCON GmbH, Ingenieurbüro für Schall- und Schwingungstechnik, Gewerbering 5, 86926 Greifenberg, Germany, markus.petz@accon.de)

Noise mapping projects for the German Federal States Baden-Wuerttemberg, Hesse, and Schleswig-Holstein and for the cities Munich, Frankfurt, and Wiesbaden are presented. These large projects have been performed using newest software technology like fully automated parallel computing applying the PCSP technique (Program Controlled Segmented Processing) and by using all CPU cores of the noise mapping computer cluster (multithreading). With the noise mapping for all main roads and railways in Austria the 64 Bit technology has been applied - this allows to handle even files of more than $10 \mathrm{~GB}$ without the need of manual intervention. The influence of these techniques on uncertainties is discussed using the strategy of DIN 45687 and it is shown that such complete 3D models are the optimal basis to develop noise reduction measures in the frame of Action Planning.

\section{3:00}

1pNSc7. Noise mapping for large urban areas - the city of Vienna. Werner Talasch (Magistrat der Stadt Wien - Magistratsabteilung 22, Dresdner Straße 45, A-1200 Wien, Austria, office@talasch.at)

The Environmental Noise Directive 2002/49/EG requires noise maps for the main noise sources railway, road and aircraft and as well for agglomerations. For agglomerations there are also industrial sites (restricted to IPPC plants) a relevant noise source. The noise maps for the agglomeration of Vienna includes the City of Vienna and 5 further municipalities. These are Brunn am Gebirge, Maria Entersdorf, Mödling, Perchtoldsdorf, and Wiener Neudorf. The area of the agglomeration is about $460 \mathrm{~km}^{2}$. The most difficult part in the task of creating the noise maps was the 3D model of the agglomeration. Most of the relevant objects were available as 3D objects. Only some objects are only available as 2D objects and caused therefore a lot of work.

\section{3:20-3:40 Break}

3:40

1pNSc8. The United Kingdom noise mapping experience. Nick Hawkins (ACCON UK Limited, Unit B, Fronds Park, Frouds Lane, Aldermaston, RG7 4LH Reading, UK, nick.hawkins@accon-uk.com), Graham Parry (ACCON UK Limited, Unit B, Fronds Park, Frouds Lane, Aldermaston, RG7 4LH Reading, UK, graham.parry@accon-uk.com)

The paper describes the noise mapping of the Bristol and Bournemouth agglomerations for the United Kingdom Noise Mapping Initiative in compliance with the European Directive on Environmental Noise and the challenges of delivering both compliant noise mapping, noise mapping outputs and shape files, and the associated methodology reports. The paper examines the noise model utilised for the mapping, the various efficiencies built into the system to provide error checking, the external data acquisition and the effects that any uncertainties may have in strategic noise mapping. Additionally, the use of a GIS based toolkit for identifying and amending the location of noise barrier objects was utilised. 


\section{Contributed Paper}

\section{4:00}

1pNSc9. A comparison between predicted and measured noise levels at the Rijeka - Zagreb motorway. Sanja Grubesa (Faculty of EE and Computing, Unska 3, Department of Electroacoustics, HR-10000 Zagreb, Croatia, sanja.grubesa@fer.hr), Marko Horvat (Faculty of EE and Computing, Unska 3, Department of Electroacoustics, HR-10000 Zagreb, Croatia, marko.horvat@fer.hr), Hrvoje Domitrovic (Faculty of EE and Computing, Unska 3, Department of Electroacoustics, HR-10000 Zagreb, Croatia, hrvoje .domitrovic@fer.hr)

The current calculations used for predicting the levels of road traffic noise can and usually do give results that can differ significantly from the results of measurements of noise levels performed in the field. In order to examine these discrepancies, levels of road traffic noise have been measured on two locations on the Rijeka - Zagreb Motorway. After that, the geographical layout of these locations has been prepared and then input into a computer simulation program with the goal of obtaining simulated road traffic noise levels. Following the assumption that these results will be different, the goal is to determine which of the simulation models used today will provide results that are in the best agreement with the results obtained from the actual field measurements, with the emphasis on this particular geographic region, namely, the Republic of Croatia.

\section{Invited Paper}

\section{4:20}

1pNSc10. City noise mapping without traffic data. Laurent Droin (Acouphen-Environnement, Campus de La Doua, 66 BD Niels Bohr, BP 52132, 69603 Villeurbanne, France, laurent.droin@acouphen-environnement.com), Pierre Odent (MVAConsultancy, 12-14 rue Jules Cesar, 75012 Paris, France, podent@mvaconsultancy.com)

The authers have a large experience on transport and noise planning and mapping with projects on many communities of different size in France. Within the time scale of a mapping project, it is very common to have a large variation of the accuracy in traffic data, especially for roads (largest source data base). In many cases, no traffic data is available on less exposed roads. This paper illustrates how variation of input data accuracy and lack of data were tackled in both large and small territories (population between 50000 and $1000000)$ in order to provide maps with the required accuracy and information for political action and communication.

\section{Contributed Papers}

\section{4:40}

1pNSc11. Definition of traffic scennaries, application on a practise case of the criteria followed by the guide of good practises for elaboration of strategic traffic noise maps in urban routes. Igone García Pérez (Labein-Tecnalia, C/Geldo-Parque Tecnologico de Bizkaia, 48160 Derio, Spain, igarcia@labein.es), Itziar Aspuru Soloaga (Labein-Tecnalia, C/Geldo-Parque Tecnologico de Bizkaia, 48160 Derio, Spain, iaspuru @labein.es)

Configuration of noisy environment in towns mostly depends upon the characterization of traffic noise in urban routes. Frequently there is a lack of information when it is a question of having data from the whole urban area. To solve this lack of information the above-mentioned guide (WG-AEN 002 .2006) proposes some suppositions for this characterization which can turn out to be very general in practical applications. Through the presentation of these criteria applied to a practical case it can be seen the tools necessary to adjust real traffic characterization to the elaboration of noise maps and definition of an Action Plan. The analyzed variables are: AVT, \% of heavy vehicles, time distribution and speed considering, for this last factor, the lacks shown by the interim method and the applicable supposes based upon the state of the art.

\section{5:00}

1pNSc12. Urban noise mapping - an approach to the establishment of standard making procedure. Predrag Vukadin (Brodarski institut d.o.o., Avenija V.Holjevca 20, 10020 Zagreb, Croatia, prevuk@hrbi.hr), Ivan Bublic (Brodarski institut d.o.o., Avenija V.Holjevca 20, 10020 Zagreb, Croatia, ivan.bublic@hrbi.hr), Ivan Tudor (Brodarski institut d.o.o., Avenija V.Holjevca 20, 10020 Zagreb, Croatia, ivan.tudor@hrbi.hr)

The Directive 2002/49 relating to the assessment and management of environmental noise and the Croatian legislative derived from it defines the basic rules to carry out strategic noise mapping. However, in real life situations, each mapping project implies a different approach, depending on various case related distinctions e.g. availability and quality of input data, extent of noise annoyance, the form and the volume of output data to be presented, etc. Based on the experience from various urban noise mapping projects, this work is an approach to defining key steps in urban noise map production and establishing standard step-by step procedure, and an attempt to define the procedure for each step. Examples for each step are given based on the case studies from our previous urban noise mapping projects. 


\title{
Session 1pNSd
}

\section{Noise and EURONOISE: Environmental Noise Mapping I}

\author{
Kenneth Cunefare, Cochair \\ Woodruff School of Mechanical Engineering, The Georgia Inst. of Technol., Atlanta, GA 30332 USA \\ Gaetano Licitra, Cochair \\ ARPAT - Dept. Firenze, Via Porpora, 22, Firenze, 50144, Italy
}

\section{Invited Papers}

$5: 40$

1pNSd1. Differences among European noise mapping methods. Stylianos Kephalopoulos (European Commission, via e. fermi, 1, 21020 Ispra, Italy, Stylianos.Kephalopoulos@jrc.it), Marco Paviotti (European Commission, via e. fermi, 1, 21020 Ispra, Italy, marco.paviotti@jrc.it)

This article will present the differences among noise maps of Lden and Lnight levels produced following the EU Interim methods and those produced following other national methods used in the EU MS. Lden and Lnight are the two indicators required by the European Environmental Noise Directive (2002/49/EC) defined at the position of $4 \mathrm{~m}$ height and $2 \mathrm{~m}$ away from the facade of a building. Four different comparisons will be presented for road, railway, industrial, and aircraft noise. This article will also focus on the methodology used to assess the equivalence among the national methods against the interim ones as well as the statistical approach used to consider uncertainties. Finally, the input values used, the parameters and the lay-outs of the four protocols corresponding to the four environmental noise sources (i.e., road traffic, railway, industrial, and aircraft noise) adopted for the equivalence exercise will be also presented.

\section{6:00}

1pNSd2. Challenges and opportunities for noise mapping in the United States. Kenneth Kaliski (Resource Systems Group (RSG), 55 Railroad Row, White River Junction, VT 05001, USA, kkaliski@ @rginc.com)

Primarily through the impetus of European Union Directive 2002/49/EC, EU cities have been on the forefront of noise mapping and the community planning that results from it. The United States has no similar legislation at federal or state levels. As a result, noise mapping for towns and cities in the U.S., and indeed, awareness of noise issues by planners, appears to lag behind their European counterparts. However, metropolitan areas in the U.S. have invested a great deal in transportation modeling. As a result, the data foundation for noise mapping, that is, road and rail geometry and traffic volumes, is in place in many metropolitan areas. In addition, digital terrain elevation data, aerial photography, and GIS data are generally available on a state or national basis at no charge over the internet. The Chittenden County, Vermont noise map is one example of how publically available data can be used to create a useful community noise map over a large area. Other examples will be discussed.

6:20

1pNSd3. Presenting Noise Mapping Information to the Public. John F. Hinton (Birmingham City Council, Regulatory Services, 581 Tyburn Road, B24 9RF Birmingham, UK, bham.acoustics@dsl.pipex.com), Soren Rasmussen (Cowi-Odense, Odensevej 95, Postboks 444, Hjallese, DK-5260 Odense, Denmark, SRS@cowi.dk)

Directive 2002/49/EC of the European Parliament and of the Council of 25 June 2002 relating to the assessment and management of environmental noise requires Member States to undertake strategic noise mapping. It also refers to legislation regarding access to the information from strategic noise maps, since it requires "that the strategic noise maps ... are made available and disseminated to the public in accordance with relevant Community legislation ... and in conformity with Annex IV ... to this Directive." Under its terms of reference for 2007 the European Environment Agency's (EEA) Working Group on the Assessment of Exposure (WG-AEN) was asked to produce a Position Paper containing the Group's ideas and suggestions on good practice and associated strategies for presenting noise mapping information to the public at local and national levels. Many of these ideas and suggestion were developed from the outcomes of a workshop held in London in October, 2006. The final draft of the Position Paper was completed in December, 2007. This conference paper and associated verbal presentation will provide an overview of the contents of the Position Paper and report on developments since December, 2007. 
1pNSd4. Limits and advantages of Good Practice Guide to Noise Mapping. Gaetano Licitra (ARPAT - Dept. Firenze, Via Porpora, 22, 50144 Firenze, Italy, g.licitra@arpat.toscana.it), Gianluca Memoli (Imperial College London, Department of Chemical Engineering, Exhibition road, SW7 2AZ London, UK, g.memoli@imperial.ac.uk)

The Pisa Noise Mapping Project has recently presented to the public what turned out to be the first noise map for road traffic in Italy, developed taking into account the Good Practice Guide version 2 (GPG2) of WG-AEN and the main results of the IMAGINE project. This paper will discuss the results of this noise map, relative to road traffic, in terms of Lden and Lnight and their uncertainties, obtained by comparing the calculated values with a set of noise measurements taken across the territory. The uncertainties so defined were compared with the ones predicted by GPG2 considering, in particular, two different ways to model the source. To do this, input traffic flow were assigned first by taking direct measurements and performing a road classification and then, at a second stage, using a static traffic model (the latter method should give less uncertainty, according to GPG2). The expected change in uncertainty will be discussed, together with advantages and disvantages of the two different choices. A comparison of exposed population with other EU realities will be also presented.

\title{
Contributed Paper
}

7:00

1pNSd5. A noise mapping study of a large metropolitan city with a heterogeneous traffic. Ramachandra Alur (Indian Institute of Technology, Acoustics Laboratory, Dept of Civil Engg, IIT- M, 600036 Chennai, India, ram_alur@yahoo.com), Kalaiselvi Ramasamy (Indian Institute of Technology, Acoustics Laboratory, Dept of Civil Engg, IIT- M, 600036 Chennai, India,kalai_archi@yahoo.com)

Traffic noise characteristics in some of the cities in a developing country like India are slightly varied by virtue of the fact that the composition of the traffic is heterogeneous accompanied by variance in road geometry and varying density of the buildings on the either side of the road. To study the feasibility of noise reduction in some of the areas a noise mapping study has been attempted along with field measurements of L10, L50, L90 and Leq. In the noise mapping parameters such as Ld, LN, Lden have been arrived at by taking into consideration the geometrical features of the roads and varying heights of the buildings. In this study noise mapping through computer simulation model (soundplan) is used by considering several noise sources and propagation of noise to the receiver point. This paper describes how the local characteristics of the city affect the propagation of noise and describes an integrated approach to control noise pollution in these areas. A three dimensional acoustical model has been developed for two locations of the city. Methodologies of noise control through noise contours around traffic are illustrated.

MONDAY AFTERNOON, 30 JUNE 2008

AMPHI HAVANE, 1:00 TO 7:40 P.M.

\section{Session 1pPAa}

\section{Physical Acoustics: Acoustics of Porous Media I}

\author{
Walter Lauriks, Cochair \\ Lab. ATF, Katholieke Universiteit Leuven, Celestijnenlaan 200D, Leuven, B-3001, Belgium
}

Keith Attenborough, Cochair

Open University, Department of Design, Development, Materials and Environment, Walton Hall, Milton Keynes, MK7 6AA, UK

\section{Invited Papers}

1pPAa1. Nonlinear acoustic acceleration waves in porous media flow. Pedro M. Jordan (U.S. Naval Research Lab., Code 7181, Stennis Space Center, MS 39529-5004, USA, pjordan@nrlssc.navy.mil)

Acoustic acceleration waves are defined as jumps in the first derivatives of the velocity, pressure, or density across a propagating singular surface (or wavefront). In this talk, the temporal evolution of the amplitude and the propagation speed of such waves are investigated in the context of finite-amplitude acoustic propagation in Darcy-type porous media. It is shown that there exists a critical value, $\alpha^{*}(>0)$, of the initial jump amplitude such that the acceleration wave magnitude either goes to zero, as $t \leftarrow \infty$, or blows up, in finite time, depending on whether the given initial jump amplitude is less than or greater than $\alpha^{*}$. In addition, a connection to traveling wave solutions is noted and the linearized case is examined. Finally, the numerical solution of a (1D) nonlinear IBVP involving sinusoidal signaling in a fluid-saturated porous slab is used to illustrate the finite-time transition from acceleration wave to shock wave, which occurs when the initial jump amplitude is greater than $\alpha^{*}$. [Supported by ONR/NRL funding (PE 061153N).] 
1pPAa2. New equipment for the measurements of flow resistivity and porosity of open cell ceramic and metal foams. Benjamin Jados (Illinois Institute of Technology, Civil, Architectural and Environmental Engineering, 3201 S. Dearborn St., Room 228, Chicago, IL 60616, USA, jadoben@iit.edu), Ralph T. Muehleisen (Illinois Institute of Technology, Civil, Architectural and Environmental Engineering, 3201 S. Dearborn St., Room 228, Chicago, IL 60616, USA, muehleisen@iit.edu)

Two of the most important measurements in the acoustic analysis of porous media are the flow resistivity and porosity. To reduce the errors in the measurements of these parameters, new instruments have been developed, which incorporate recent technological advances. The new equipment helps to streamline the user experience and improve the confidence of the results. The current design includes the use of LabVIEW and COMSOL Multiphysics software, the use of thermal press-fit sample holders, and the use of low differential pressure transducers. The new instruments were used to study the flow resistivity and porosity of various pore sizes of open cell ceramic and metal foams. Data are presented with accompanying errors, as well as a discussion of the shortcomings and possible improvements of the measurement system.

\section{$1: 40$}

1pPAa3. Semi-empirical time domain model of sound attenuation in porous materials. Olga Umnova (University of Salford, Acoustics Research Centre, Newton Building, M5 4WT Salford, UK, o.umnova@salford.ac.uk), Diego Turo (University of Salford, Acoustics Research Centre, Newton Building, M5 4WT Salford, UK, d.turo@pgr.salford.ac.uk)

A semi-empirical model for complex tortuosity function, which satisfies physically correct low and high frequency limits and allows analytical transformation into the time domain has been developed. It is based on the assumption, that a network of pores with two characteristic sizes can approximate the internal structure of the material, and thus requires the knowledge of two relaxation times. It is proven, however, that the model can predict sufficiently well the acoustical properties of rigid porous materials with various microstructures when is complete with the tortuosity as an additional parameter. It is shown that relaxation times can be easily related to the "equivalent fluid" model parameters. Numerical time domain calculations confirm that the contribution of both viscous and inertial effects on the evolution of acoustical pulses as they propagate through a porous material can be accounted for simultaneously within the framework of the model. It is shown that the extended version of the model, which accounts for the thermal effects can also be explicitely used for time-domain computations.

\section{2:00}

1pPAa4. Acoustical and micro-structural properties of recycled grains and fibres. Kirill V. Horoshenkov (University of Bradford, School of Engineering, Design and Technology, BD7 1DP Bradford, UK, k.horoshenkov@ Bradford.ac.uk), Amir Khan (University of Bradford, School of Engineering, Design and Technology, BD7 1DP Bradford, UK, a.khan72@bradford.ac.uk), Hadj Benkreira (University of Bradford, School of Engineering, Design and Technology, BD7 1DP Bradford, UK, h.benkreira@bradford.ac.uk), Giulio Pispola (Umbra Group, Umbra Cuscinetti S.p.A., Zona Industriale, Localita Paciana, 06034 Foligno, Italy, gpispola@umbracus.com)

This paper presents a systematic study into the production, characterisation and modeling of the acoustic behaviour of highly heterogeneous, low density porous layers having a complex pore size distribution. A new cold extrusion production method was developed at the University of Bradford to process recycled polymeric fibres and grains so that accurate control of the pore size distribution and the porosity of the resultant porous product could be attained. In this way high values of the acoustic absorption coefficient could be obtained in a relatively thin porous layer throughout the design frequency range. Two approaches were used to model the acoustic performance of the manufactured porous media. The first approach requires the direct numerical integration of the Biot viscosity correction function which depends on the probability density function of the pore size. The other approach assumes a low permeability contrast between the two porous scales so that the acoustic properties could be estimated using the semi-phenomenological models of Johnson and Lafarge for the viscous and thermal dynamic permeabilities. Numerical results predicted by the two models were then compared with impedance tube experimental data showing good accuracy of the selected prediction methods.

1pPAa5. Deduction of porous material properties using a point source. Jean François Allard (Laboratoire d'Acoustique de l’Université du Maine, Avenue Olivier Messiaen, 72085 Le Mans, France, jean-francois.allard@univ-lemans.fr), Olivier Dazel (Laboratoire d'Acoustique de l'Université du Maine, Avenue Olivier Messiaen, 72085 Le Mans, France, olivier.dazel@univ-lemans.fr), Laurens Boeckx (Lab. ATF, Katholieke Universiteit Leuven, Celestijnenlaan 200D, B-3001 Leuven, Belgium, laurens.boeckx@fys.kuleuven.be), Nathalie Geebelen (Lab. ATF, Katholieke Universiteit Leuven, Celestijnenlaan 200D, B-3001 Leuven, Belgium, Nathalie.Geebelen@bwk.kuleuven.be), Walter Lauriks (Lab. ATF, Katholieke Universiteit Leuven, Celestijnenlaan 200D, B-3001 Leuven, Belgium, Walter.Lauriks@fys.kuleuven.be)

The acoustic field created by a point source above the plane boundary between two semi-infinite fluid layers has been previously studied by Brekhovskikh. A straightforward generalization can be performed by replacing the fluid under the source by the fluid equivalent to a rigid-framed porous medium. This leads to a method of evaluating, from pressure measurements, the surface impedance close to grazing incidence and the Brewster angle of total refraction of the porous medium. The model by Brekhovskikh can also be generalized when a thin porous layer is set under the point source. A pole of the reflection coefficient exists for an angle of incidence close to grazing incidence. This pole is related to an acoustic field similar to a surface wave above the layer. The measurement of this angle provides an evaluation of the surface impedance close to grazing incidence. The point source induces frame vibrations that can be predicted with the Biot theory. The rigidity coefficients of the frame at audible frequencies can be evaluated from measurements of the frame velocity with a laser velocimeter. 
1pPAa6. Acoustic waves propagation along mechanically free surface of unconsolidated granular porous media. Xavier Jacob (LPEC/UMR 6087/CNRS/Université du Maine, Avenue Olivier Messiaen, 72085 Le Mans Cedex 09, France, xavier.jacob@univ-lemans.fr), Vladislav Aleshin (LPEC/UMR 6087/CNRS/Université du Maine, Avenue Olivier Messiaen, 72085 Le Mans Cedex 09, France, aleshinv@mail.ru), Vincent Tournat (LPEC/UMR 6087/CNRS/Université du Maine, Avenue Olivier Messiaen, 72085 Le Mans Cedex 09, France, vincent.tournat@univ-lemans.fr), Philippe Leclaire (Lab. de Recherche en Mécanique et Acoustique, I.S.A.T - Univ. de Bourgogne, 49, rue Mademoiselle Bourgeois, 58000 Nevers, France, philippe.leclaire@gmail.com), Walter Lauriks (Lab. ATF, Katholieke Universiteit Leuven, Celestijnenlaan 200D, B-3001 Leuven, Belgium, Walter.Lauriks@fys.kuleuven.be), Vitali Gusev (LPEC/UMR 6087/CNRS/Université du Maine, Avenue Olivier Messiaen, 72085 Le Mans Cedex 09, France, vitali.goussev@univ-lemans.fr)

Unconsolidated granular materials exhibit strong dependence of elastic properties on pressure due to high sensitivity of the intergrain contacts to magnitude of loading. As a consequence the gravity field makes these granular assemblages highly elastically inhomogeneous particularly near mechanically free surface. Theoretical and experimental investigations conducted recently have demonstrated that multiple waveguide surface acoustic modes propagate along the free surface of the solid-state skeleton in the disordered air-saturated granular packings. These modes are localized near the surface. In ordered granular packing the acoustic waves can travel along the surface inside the horizontal channels, which are localized beneath the surface. The waves of different frequencies are travelling at different depths. The anisotropy of these inhomogeneous granular phononic crystals has an important influence of the existence of the waveguide modes. The experiments with acoustic waves traveling in the vicinity of a free surface provide fundamental information on the mechanical behaviour of unconsolidated granular media at very low pressures in the vicinity of the jamming transition. The perspectives of this research could be related to the analysis of the role which could be played in the considered phenomena by the acoustic waves predominantly travelling in these granular porous assemblages through the fluid-saturated pores.

\section{3:00}

1pPAa7. The Biot Type II wave in the fluid and matrix of soils. James M. Sabatier (University of Mississippi, NCPA, 1 Coliseum Drive, University, MS 38677, USA, sabatier@olemiss.edu), Chris McNeill (University of Mississippi, NCPA, 1 Coliseum Drive, University, MS 38677, USA, cmcneil1@olemiss.edu)

When an acoustic wave is incident on the surface of the porous ground energy is coupled into the soil matrix and fluid. To investigate the depth dependence of the Type I and II waves from the ground surface, the ground is modeled as a semi-infinite air-filled poroelastic medium. Using a modified form of Biot-Stoll theory the magnitude and phase of the matrix velocity and fluid pressure caused by the two possible dilatational waves are determined. Traditionally speaking the type I (fast) wave is the nondispersive wave traveling in the solid, and the type II (slow) wave is dispersive and travels primarily in the fluid (air). Previous experiments have shown the possibility that the type II wave travels in the solid near the surface and influences the measured matrix velocity and fluid pressure. Calculations have been done showing that near the surface of the ground the type II wave actually does have a large effect on the particle velocity of the matrix. After the first few centimeters the type II wave is completely attenuated and the type I wave is responsible for the displacement and pressure in the medium

1pPAa8. Anisotropy effects on the acoustical properties of porous materials. Olivier Dazel (Laboratoire d'Acoustique de l’Université du Maine, Avenue Olivier Messiaen, 72085 Le Mans, France, olivier.dazel@univ-lemans.fr), Jean François Allard (Laboratoire d'Acoustique de l'Université du Maine, Avenue Olivier Messiaen, 72085 Le Mans, France, jean-francois.allard@univ-lemans.fr), Laurens Boeckx (Lab. ATF, Katholieke Universiteit Leuven, Celestijnenlaan 200D, B-3001 Leuven, Belgium, laurens.boeckx@fys.kuleuven.be), Nathalie Geebelen (Lab. ATF, Katholieke Universiteit Leuven, Celestijnenlaan 200D, B-3001 Leuven, Belgium, Nathalie.Geebelen@bwk.kuleuven.be), Poonam Khurana (Lab. ATF, Katholieke Universiteit Leuven, Celestijnenlaan 200D, B-3001 Leuven, Belgium, Poonam.Khurana@fys.kuleuven.be), Walter Lauriks (Lab. ATF, Katholieke Universiteit Leuven, Celestijnenlaan 200D, B-3001 Leuven, Belgium, Walter.Lauriks@fys.kuleuven.be)

Porous materials are now widely used in noise control for their acoustic properties in sound absorption and transmission. These properties are function of the internal porous medium structure. Generally, most of the models assume that the porous medium is isotropic. Mineral wools (as well as some foams) clearly present an anisotropic structure. This communication is concerned with the adaptation of recent works on isotropic materials to the case of anisotropic porous materials. The general theory will be exposed for sound absorbing materials with anisotropic acoustical and mechanical parameters. The case of transverse isotropic materials (with fibers organized in planes running parallels one to each other) will then be detailed in particular in the case when the plane of fibers is not the same than the normal plane of the sample. The influence on the acoustical properties anisotropic porous materials will then be presented. Illustrations are then provided with measurements performed on wools and it will be shown that the rigidity coefficients of a sample can be evaluated at audible frequencies from measurements in particular from the speed of the Rayleigh wave in different directions. 


\section{Contributed Papers}

3:40

1pPAa9. Low frequency implementation of the full Kramers-Kronig relationships for the description of dispersion and attenuation of acoustical waves in porous media. Bernard R. Castagnede (Laboratoire d'Acoustique de l'Université du Maine, Avenue Olivier Messiaen, 72085 Le Mans, France, bernard.castagnede@univ-lemans.fr), Denis Lafarge (Laboratoire d'Acoustique de l'Université du Maine, Avenue Olivier Messiaen, 72085 Le Mans, France, denis.lafarge@univ-lemans.fr), Claude_Depollier (Laboratoire d'Acoustique de l'Université du Maine, Avenue Olivier Messiaen, 72085 Le Mans, France, claude.depollier@univ-lemans.fr), Naima Sebaa (Laboratoire d'Acoustique de l'Université du Maine, Avenue Olivier Messiaen, 72085 Le Mans, France, naima.sebaa@univ-lemans.fr), Michel Henry (Laboratoire d'Acoustique de l'Université du Maine, Avenue Olivier Messiaen, 72085 Le Mans, France, michel.henry@univ-lemans.fr)

It is well known that the causality principle which applies during the acoustic propagation of a wave packet inside any material should be described by the universal Kramers-Kronig (K-K) relationships, enabling to formally link dispersion to attenuation curves. The application of the K-K formalism to porous networks has been studied lately, but unfortunately, the metrology of dispersion features at very low frequency is intricate. The use of parametric arrays of nonlinear acoustics enables to get at the same time, some precise information on dispersion and absorption on various porous media, for instance in the frame of the "equivalent fluid" model. In the present work, we describe some very recent findings obtained along these ideas for a truly relevant application of the full KK formalism in various fibrous materials. We have checked numerical predictions of the absorption versus frequency computed from the dispersion curves with the K-K modeling, as well as confrontation of these numerical predictions with experimental data on absorption obtained with various set-ups, on different fibrous materials.

\section{4:00-4:20 Break}

\section{$4: 20$}

1pPAa10. On the diffusion of sound energy in porous materials Nicola Prodi (Engineering Dept. - Univ. of Ferrara, Via Saragat, 1, 44100 Ferrara, Italy, nicola.prodi@unife.it)

The sound intensity was used in the past to describe the surface properties of porous materials. Actually no extensive study used the same concepts to describe the properties of the energy flow inside the material. As known, the sound intensity has a twofold nature, that is an active part transferring the energy out of a point and a reactive one localizing the energy. These features are crucial in the development of the new model for the propagation of the energy inside porous materials developed in this work. In fact, by means of the intensimetric approach, it is shown that the one-dimensional transfer of sound energy can be fully described as a diffusion process. The role of the active and reactive sound intensities is outlined and specific parameters are defined to account for the material behaviour with respect to the transfer of the sound energy. Moreover the sinks of energy and the sources of reactivity in the material are derived and discussed.

\section{4:40}

1pPAa11. Optimal sound absorbing and manufacturable two-dimensional, hexagonal-like porous structure. Camille Perrot (Groupe d'Acoustique de l'Université de Sherbrooke, 2500, Boul. de 1'Université, Département de génie mécanique, Sherbrooke, QC J1K-2R1, Canada, camille.perrot@usherbrooke.ca), Fabien Chevillotte (Groupe d'Acoustique de l'Université de Sherbrooke, 2500, Boul. de l'Université, Département de génie mécanique, Sherbrooke, QC J1K-2R1, Canada, fabien.chevillotte@usherbrooke.ca), Raymond_Panneton (Groupe d'Acoustique de l'Université de Sherbrooke, 2500, Boul. de l'Université, Département de génie mécanique, Sherbrooke, QC J1K-2R1, Canada, raymond.panneton@usherbrooke.ca)

Results from a numerical study examining micro-macro relations linking local geometry parameters to sound absorption properties are presented. For an hexagonal structure of solid fibers, the porosity $\phi$, the thermal characteristic length $\Lambda^{\prime}$, the static viscous permeability ko, the tortuosity $\alpha_{\infty}$, the viscous characteristic length $\Lambda$, and the sound absorption coefficient are computed. Numerical solutions of the steady Stokes and electrical equations are employed to provide ko, $\alpha_{\infty}$, and $\Lambda$. Hybrid estimates based on direct numerical evaluation of porosity $\phi, \Lambda^{\prime}, \mathrm{ko}, \alpha_{\infty}, \Lambda$ and the analytical model derived by Johnson, Champoux, and Allard are used to relate varying (i) throat size, (ii) pore size, and (iii) fibers cross-section shapes to the sound absorption spectrum. The result of this paper tends to demonstrate the important effect of throat size in the sound absorption level, cell size in the sound absorption frequency selectivity, fibers cross-section shape in the porous material weight reduction. In a hexagonal porous structure with solid fibers, the sound absorption level will tend to be maximized with a $48 \pm 10$ $\mu \mathrm{m}$ throat size corresponding to an intermediate resistivity, a $13 \pm 8 \mu \mathrm{m}$ fiber radius associated with small pores, and convex triangular cross-section shape fibers allowing weight reduction.

\section{5:00}

1pPAa12. Analytical microstructural model for acoustical porous materials with single or double porosity. Emmanuel Gourdon (Université de Lyon, Ecole Nationale des Travaux Publics de l'Etat, CNRS, URA 1652, Département Génie Civil, 3, rue Maurice Audin, F-69120 Vaulx-en-Velin, France, emmanuel.gourdon@entpe.fr), Luc Jaouen (Matelys - Acoustique \& Vibrations, 20/24 rue Robert Desnos, 69120 Vaulx-en-Velin, France, luc.jaouen@matelys.com)

An analytical model of sound propagation for porous materials with single or double scale of porosity is described. For each scale, pores and interconnections between them are modeled by a serie of two cylinders; a cylindrical periodical cell is thus considered. Scales are supposed to be separated, the porous medium is supposed to be periodic and to have a motionless skeleton. The geometrical parameters needed to quantify visco-thermal effects are directly related to the microstructure of the material. These parameters: lengths and radii of pores and interconnections can be extracted from image analysis for example. From additional conditions on cell morphology, independent parameters per porosity scale can be reduced to a number of three. Good comparisons between theoretical calculations of the sound absorption coefficient at normal incidence and impedance tube measurements are obtained for single and double porosity (meso-perforated) materials.

\section{$5: 20$}

1pPAa13. Adsorption in activated carbon and its effects on the low frequency performance of hearing defenders. Fouad Bechwati (University of Salford, Acoustics Research Centre, Newton Building, M5 4WT Salford, UK, f.bechwati@pgr.salford.ac.uk), Trevor J. Cox (University of Salford, Acoustics Research Centre, Newton Building, M5 4WT Salford, UK, t.j.cox@ @alford.ac.uk), Mark R. Avis (University of Salford, Acoustics Research Centre, Newton Building, M5 4WT Salford, UK, m.r.avis@salford.ac.uk), Olga Umnova (University of Salford, Acoustics Research Centre, Newton Building, M5 4WT Salford, UK, o.umnova @ salford.ac.uk)

Activated carbon displays interesting behaviours at low frequencies due to its large internal surface area and complex network of pores of various sizes and shapes. The material can produce larger than expected absorption and change the compliance of acoustic enclosures. This paper investigates the performance of hearing defenders which utilize activated carbon as the lining material of the cup. Compared to a standard foam liner, the introduction of activated carbon increases the insertion loss by up to 15 to $20 \mathrm{~dB}$ at frequencies between 31.5 and $250 \mathrm{~Hz}$. The enhanced insertion loss is due to the increase in stiffness and apparent volume of the cup cavity as the activated carbon is introduced. This is probably due to the substantial change in local density as air molecules adsorb onto, and desorb from, the activated carbon pores during sound propagation. There is a change in entropy and energy loss from the sound wave during the adsorption/ desorption process due to the existence of a hysteresis loop. This additional absorption enhances performance. 


\section{5:40}

1pPAa14. Ultrasonic behaviour of high pressure air filled porous media. Stephane Griffiths (LAUM, CNRS, Université du Maine, Av. O. Messiaen, 72085 Le Mans, France, stephane.griffiths@live.fr), Christophe Ayrault (LAUM, CNRS, Université du Maine, Av. O. Messiaen, 72085 Le Mans, France, christophe.ayrault@univ-lemans.fr)

This paper deals with the acoustic behaviour of porous media when the saturating fluid is high pressured. These observations are performed by ultrasonic transmission through a porous sample with variations of the static pressure of the saturating fluid. In order to characterize high damping materials, measurements are performed for high static pressure (up to 18 bars). It is shown that the behaviour of transmission coefficient and speed with pressure follow the Biot's theory. Moreover, measurements are strongly dependant on temperature, which is not visible in modelizations with Biot's model, although this parameter is taken into account in thermodynamical parameters. It is therefore assumed that mechanical characteristics vary with temperature and pressure. An estimation of mechanical parameters is then performed by minimization between the Biot's model and experimental data. First results, obtained in suitable cases for which measurements quality is good and minimization process converges correctly, show that mechanical parameters follow the evolution with frequency described by Pritz at low frequencies. Further researches are still necessary to determine dependance to temperature.

\section{6:00}

1pPAa15. Automatic characterisation of ground surfaces from in situ measurements. Guillaume Dutilleux (Lab. Régional des Ponts et Chaussées, 11, rue Jean Mentelin, BP 9, 67035 Strasbourg Cedex 2, France, Guillaume.Dutilleux@equipement.gouv.fr), David Ecotiere (Lab. Régional des Ponts et Chaussées, 11, rue Jean Mentelin, BP 9, 67035 Strasbourg Cedex 2, France, david.ecotiere@equipement.gouv.fr)

The study of long term acoustic performance of road pavements requires to access the evolution of the intrinsic parameters of these surfaces, such as resistivity or tortuosity. In long range sound propagation as well, it is well known that ground absorption is time varying. The understanding of this variation is an important issue. In both application cases, nondestructive measurement methods are required. The present contribution focuses on the 2-parameter model by Delany and Bazley for outdoor surfaces and the 4-parameter one by Hamet-Berengier for porous road pavements. It is shown that for both models the absorption coefficient spectrum obtained by an ISO 13472-1 compliant measurement contains enough data for the automatic identification. For the 2-parameter model the identification is based on a suitable cost function. The complexity of the function is low enough for a resolution by exhaustive search. It turns out that, for a large set of soft ground samples, the cost function is convex. Therefore the resolution can be done quite efficiently by classical gradient descent methods. Regarding the 4-parameter model, although the cost function is no longer convex, the resolution can be performed using a global optimization tool, like an evolutionary algorithm.

\section{6:20}

1pPAa16. A new setup for measuring the mechanical properties of porous materials. Nicolas Dauchez (Laboratoire d'Acoustique de l'Université du Maine, Avenue Olivier Messiaen, 72085 Le Mans, France, nicolas.dauchez@univ-lemans.fr), Olivier_Doutres (Laboratoire d'Acoustique de l'Université du Maine, Avenue Olivier Messiaen, 72085 Le Mans, France, olivier.doutres.etu@univ-lemans.fr), Jean Michel Genevaux (Laboratoire d'Acoustique de l'Université du Maine, Avenue Olivier Messiaen, 72085 Le Mans, France, jean-michel.genevaux@univ-lemans.fr), Guy Lemarquand (Laboratoire d'Acoustique de l'Université du Maine, Avenue Olivier Messiaen, 72085 Le Mans, France, guy.lemarquand@univ-lemans.fr)

A new device for determining complex Young's modulus of porous materials in a extended frequency range is proposed. Classical methods are based on quasitatic or dynamic response of porous material. These methods generaly neglect the coupling between the surrounding fluid and the porous frame so that they are restricted to low frequency range $(<100 \mathrm{~Hz})$ or specific sample shape. Dynamic methods provide relevant information only at the resonance frequencies of the frame. The proposed method extends the quasistatic method towards high frequencies: 1 . the porous sample is setup in a cavity in order to avoid the coupling with the external fluid, 2. a specific electrodynamic transducer has been developped to get the mechanical impedance of the sample from the measurement of the electrical impedance, 3 . mechanical properties of the frame are derived by inverse method using Biot theory so that the frequency range is not restricted to the quasistatic domain. First results obtained with a prototype validate the method in comparison with two classical methods.

\section{6:40}

1pPAa17. Quasistatic evaluation of mechanical properties of poroelastic materials: static and dynamic strain dependence and in vacuum tests. Paolo Bonfiglio (Dipartimento di Ingegneria - University of Ferrara, Via Saragat 1, 44100 Ferrara, Italy, paolo.bonfiglio@unife.it), Francesco Pompoli (Dipartimento di Ingegneria - University of Ferrara, Via Saragat 1, 44100 Ferrara, Italy, francesco.pompoli@unife.it), Paresh Shravage (Dipartimento di Ingegneria - University of Ferrara, Via Saragat 1, 44100 Ferrara, Italy, paresh.shravage @unife.it)

A complete description of the vibro-acoustical behavior of a poroelastic material requires the knowledge of both geometrical quantities, related to the structure of the fluid-filled pores to account the sound propagation within them, and mechanical parameters (i.e. Young Modulus, Poisson's ratio, and loss factor) in order to model the wave propagation through the elastic structure constituting its skeleton. Because the nonlinear nature of poroelastic media, those mechanical properties are shown depending on static preload and dynamic strain applied to them. In the present work a well established quasi-static method, based on the measurement of mechanical impedance and the use of adequate polynomial relations, has been used to determine the dependence of the mechanical properties on the applied deformations. Furthermore, tests have been also carried out in a vacuum chamber in order to evaluate the real contribution of the filling fluid on the total vibro-acoustical response of the material.

\section{7:00}

1pPAa18. Sound absorbing properties of materials made of rubber crumbs. Francesco Asdrubali (Università degli studi di Perugia, Via G. Duranti 67, 06125 Perugia, Italy, fasdruba@unipg.it), Francesco D’Alessandro (Università degli studi di Perugia, Via G. Duranti 67, 06125 Perugia, Italy, dalessandro.unipg @ ciriaf.it), Samuele Schiavoni (Università degli studi di Perugia, Via G. Duranti 67, 06125 Perugia, Italy, schiavo81 @ libero.it)

Recycled tyre granules can be used for manufacturing acoustic insulating and absorbing materials, with applications in buildings and road barriers. Therefore, the production of these materials is a valid alternative to the disposal into landfill or incineration of used tyres. This paper presents the results of sound absorbing coefficient measurements of several samples manufactured at the Acoustics Laboratory of the University of Perugia. The sound absorbing panels were produced by mixing rubber crumbs and an adequate binder in a proper proportion and then by compacting the obtained mix. The methodology used to evaluate coefficient of absorption coefficient is indicated in ISO 10534-2 standard, thanks to an impedance tube. The influence on the absorption performance of granules size, binder concentration, thickness and compaction ratio of the samples was investigated and an optimization process was carried out, in order to produce a sample with satisfying acoustical performances.

\section{$7: 20$}

1pPAa19. Measurement of acoustic and mechanical parameters of poroelastic materials by mean of active control and wave-based methods. Cedric Batifol (Centre Acoustique du LMFA, Ecole Centrale de Lyon, 36 avenue Guy de Collongue, 69134 Ecully cedex, France, cedric.batifol@ec-lyon.fr), Marie-Annick Galland (Centre Acoustique du LMFA, Ecole Centrale de Lyon, 36 avenue Guy de Collongue, 69134 Ecully 
cedex, France, marie-annick.galland@ec-lyon.fr),Mohamed Ichchou(Centre Acoustique du LMFA, Ecole Centrale de Lyon, 36 avenue Guy de Collongue, 69134 Ecully cedex, France, mohamed.ichchou@ec-lyon.fr)

Poroelastic materials are used in sound barriers for sound insulation and absorption. At low frequencies, the medium is modeled using the Biot theory. This homogenised model needs to be feed by at least eight parameters using the Johnson-Allard approach. The presented characterisation method relies on two specific test facilities. First, acoustic parameters are obtained thanks to a modified Kundt tube. The porosity and resistivity are obtained by direct measurements of the surface impedance with hard wall and zero pressure boundary conditions on the rear face of the sample, respectively. This last boundary conditions is achieved thanks to active control procedure. Finally, the remaining acoustic parameters are obtained in a least mean square sense. Mechanical parameters are then measured on a second test bench. A beam made of the poroelastic sample is subjected to mechanical broadband forcing. The transverse displacement is measured along the beam thanks to a laser vibrometre. Dispersion curves are then obtained by calculating the Inhomogeneous Wave Correlation (IWC) ratio. The Young modulus and loss factor of the sample are estimated thanks to an optimisation procedure applied to the finite element model of the test. Results are compared to those obtained using more classical quasi-static methods.

MONDAY AFTERNOON, 30 JUNE 2008

ROOM 351, 1:00 TO 7:00 P.M.

\title{
Session 1pPAb
}

\section{Physical Acoustics: Phononic Crystals I}

\author{
Pierre Deymier, Cochair \\ University of Arizona, Materials Science and Engineering Department, Mines Bldg., P.O. Box 210012, Tucson, AZ 85721, USA \\ Jérôme Vasseur, Cochair \\ IEMN, UMR CNRS 8520, avenue Poincaré, BP 60069, Villeneuve d'Ascq, 59652, France
}

\section{Contributed Papers}

\section{1:00}

1pPAb1. Rubber/air acoustic band gap materials: Elastic and viscoelastic effects. Bassam Merheb (University of Arizona, Materials Science and Engineering Department, Mines Bldg., P.O. Box 210012, Tucson, AZ 85721, USA, bassam @merheb.net), Pierre Deymier (University of Arizona, Materials Science and Engineering Department, Mines Bldg., P.O. Box 210012, Tucson, AZ 85721, USA, deymier@u.arizona.edu), Manish Jain (3M Corporation, Corporate Research Materials Laboratory, Bldg. 201-3N-04, 3M Center, St. Paul, MN 55144-1000, USA, mjain@mmm.com), Marie Aloshyna-Lesuffleur (3M Corporation, Corporate Research Materials Laboratory, Bldg. 201-3N-04, 3M Center, St. Paul, MN 55144-1000, USA, mlesuffleur@mmm.com), Richard Greger (3M Corporation, Corporate Research Materials Laboratory, Bldg. 201-3N-04, 3M Center, St. Paul, MN 55144-1000, USA, rgreger@mmm.com), Sanat Mohanty (3M Corporation, Corporate Research Materials Laboratory, Bldg. 201-3N-04, 3M Center, St. Paul, MN 55144-1000, USA, smohanty@mmm.com), Ali Berker (3M Corporation, Corporate Research Materials Laboratory, Bldg. 201-3N-04, 3M Center, St. Paul, MN 551441000, USA, aberker@mmm.com)

Two-dimensional elastic and viscoelastic phononic crystals structures are investigated theoretically and experimentally. These are two-dimensional arrays of cylinders of air inclusions in a solid silicone rubber matrix as well as arrays of silicone rubber cylinders in an air matrix. In this study, viscoelasticity is modeled with a compressible general linear viscoelastic fluid model. Numerical calculations of transmission spectra and band structures are conducted by extending the finite difference time domain (FDTD) method to account for linear viscoelastic materials which exhibit timedependent moduli. These systems demonstrate very wide band gaps in their transmission spectra that extend to frequencies in the audible range of the spectrum. Experimental and calculated transmission spectra for a rubber matrix/air inclusions phononic crystal show that the system behaves as a flu$\mathrm{id} /$ fluid composite with respect to the longitudinal polarization of acoustic waves due to the large contrast between the transverse and longitudinal speeds of sound. We also demonstrate that viscoelasticity can attenuate transmission over very wide ranges of frequency leaving only passing bands at very low frequency. These phononic crystals demonstrate the practical design of elastic or viscoelastic solid rubber/air acoustic band gap sound barrier with small dimensions.

\section{$1: 20$}

1pPAb2. Development of a suitable PML for an harmonic study of a finite 1D phononic crystal. Maxime Bavencoffe (LOMC FRE-3102 CNRS, Groupe Ondes Acoustiques, University of Le Havre, Place Robert Schuman, BP 4006, 76610 Le Havre, France, maxime.bavencoffe@univ-lehavre.fr), Bruno Morvan (LOMC FRE-3102 CNRS, Groupe Ondes Acoustiques, University of Le Havre, Place Robert Schuman, BP 4006, 76610 Le Havre, France, bruno.morvan@univ-lehavre.fr), Anne-Christine Hladky (IEMN, UMR CNRS 8520, avenue Poincaré, BP 60069, 59652 Villeneuve d'Ascq, France, anne-christine.hladky@isen.fr), Olivier Bou Matar (IEMN, UMR CNRS 8520, avenue Poincaré, BP 60069, 59652 Villeneuve d'Ascq, France, olivier.boumatar@iemn.univ-lille1.fr), Jean-Louis Izbicki (LOMC FRE3102 CNRS, Groupe Ondes Acoustiques, University of Le Havre, Place Robert Schuman, BP 4006, 76610 Le Havre, France, jean-louis.izbicki @univ-lehavre.fr)

This paper deals with the interaction of ultrasonic Lamb waves with a 1D phononic crystal. The studied structure is a finite plate with a periodic corrugated surface. Two types of forbidden bands arise in the Lamb wave dispersion curves. The first one is located at the limit of the first Brillouin zone, the second one exists at the crossing of dispersion curves of two different Lamb modes. These forbidden bands lead to conversion phenomena. In order to study accuratly the conversion phenomena in the band gaps, harmonic finite element analysis are performed. Perfect matching layers (PML) on both sides of the plate are then necessary to avoid stationnary waves. PML, adapted for Lamb modes involved at a given frequency, must be designed. By using these PML, the attenuation of Lamb waves propagating in the phononic crystal is clearly shown and is related to the existence of the forbidden band.

\section{1:40}

1pPAb3. Plate waves in phononic crystals slabs. Jiu-Jiu Chen (CNRS and Paris VI University, INSP - 140 rue de Lourmel, 75015 Paris, France, chen99nju@gmail.com), Bernard Bonello (CNRS and Paris VI University, INSP - 140 rue de Lourmel, 75015 Paris, France, bernard.bonello@insp .jussieu.fr)

We have computed the dispersion curves of plate waves propagating in periodic composite structures composed of isotropic tungsten cylinders 
embedded in a isotropic silicon background. The phononic crystal has a square symmetry and the calculation is based on the plane wave expansion method. The direction of propagation is defined by the angle $\varphi$ between $\Gamma \mathrm{X}$ in the reduced Brillouin zone and the wave vector k. If $\varphi=0\{0\}$ or $\varphi=90$ $\{0\}, \mathrm{SH}$ modes are simple hyperbolas and do not couple to the Lamb wave modes polarized in the sagittal plane. If is different from $0\{0\}$ or $90\{0\}, \mathrm{SH}$ modes convert to Lamb wave modes and couple with the flexural and dilatational modes. The symmetric modes splitting in the band structures along this direction and all three components of the particle displacements demonstrate this phenomenon which is different from the pure isotropic plate case where $\mathrm{SH}$ waves decouple to Lamb waves whatever the propagating direction.

\section{2:00}

1pPAb4. Analysis of bandpass spectra of phononic defect-mode waveguides based on mode coupling between point defects. Toyokatsu Miyashita (Dept. Electronics \& Informatics, Ryukoku University, Seta Oecho Yokotani 1-5, 520-2194 Otsu, Japan, miya@rins.ryukoku.ac.jp)

Pass bands of defect-mode waveguides fabricated in sonic/phononic crystals have desirable characteristics for their practical applications to acoustic bandpass filters. Their mechanism is considered to originate in mode-coupling characteristics of point defects, a chain of which composes a defect-mode waveguide. Especially, mode confinement in each point defect or their mode coupling is found to decide the flatness of the pass band. For example, with a relatively strong mode confinement, the transmission of the pass band swings between 0 and $-16 \mathrm{~dB}$ with frequency, although the transmission outside the pass band remains still typically at $-50 \mathrm{~dB}$. On the contrary with a moderate mode confinement, the transmission remains fairly flat only with a swing of $2 \mathrm{~dB}$. We have obtained these characteristics not only by an elastic FDTD method but also by acoustic experiments. These have been investigated theoretically and experimentally based on analyses of the mode coupling between point defects. Two sonic/phononic crystals are considered; one is composed of acrylic-resin cylinders in air, and the other steel cylinders in water.

\section{2:20}

1pPAb5. Design and characterization of stop-band filters using PZT layer on silicon substrate phononic crystals. Jérôme Vasseur (IEMN, UMR CNRS 8520, avenue Poincaré, BP 60069, 59652 Villeneuve d'Ascq, France, jerome.vasseur@univ-lille1.fr), Anne-Christine Hladky-Hennion (IEMN, UMR CNRS 8520, avenue Poincaré, BP 60069 59652 Villeneuve d'Ascq, France, Anne-Christine.Hladky@isen.fr), Bertrand Dubus (IEMN, UMR CNRS 8520, avenue Poincaré, BP 60069, 59652 Villeneuve d'Ascq, France, Bertrand.Dubus@isen.fr), Bahram Djafari-Rouhani (IEMN, UMR CNRS 8520, avenue Poincaré, BP 60069, 59652 Villeneuve d'Ascq, France, Bahram.Djafari-Rouhani@univ-lille1.fr), Bruno Morvan (LOMC FRE3102 CNRS, Groupe Ondes Acoustiques, University of Le Havre, Place Robert Schuman, BP 4006, 76610 Le Havre, France, bruno.morvan@univlehavre.fr)

Phononic crystals are periodic structures exhibiting absolute band gaps i.e. frequency bands in which the propagation of elastic waves is forbidden in all directions. Filtering is then a possible application of phononic crystals. Recently, the existence of absolute band gaps has also been theoretically demonstrated for guided elastic waves in a piezoelectric plate on a substrate [J. Vasseur et al, J. Appl. Phys, 101, 114904 (2007)], which is a geometry of interest for possible co-integration on silicon chip. The $2 \mathrm{D}$ phononic crystal was constituted by a square arrangement of cylindrical holes in a PZT layer deposited on a silicon substrate. In this communication, the realization of a stop-band filter constituted by a periodically patterned PZT layer, polarized along thickness, on silicon substrate and interdigitated electrodes (IDE) for emission/reception of guided elastic waves, is investigated. The filter characteristics are theoretically evaluated by using finite element simulations: dispersion curves of patterned PZT layer are computed for various pattern geometries to obtain the absolute band gap. Complete structure is then modeled, with appropriate IDE to propagate a guided mode in the piezoelectric layer. Finally, filtering capability of the structure is evaluated. Work supported by STMicroelectronics (Nano2008 program of French ministry of industry)

\section{2:40}

1pPAb6. Acoustic response of a soniclike crystal within a rigid frame porous plate. Jean-Philippe Groby (DRE/L2S - UMR8506 CNRS/Supelec/Univ. Paris Sud 11, Département de Recherche en Electromagnétisme/Laboratoire des signaux et systèmes, 3 rue Joliot-Curie, 91192 Gif-sur-Yvette cedex, France, groby@1ss.supelec.fr), Armand Wirgin (CNRS-Laboratoire de Mécanique et d'Acoustique, 31 Chemin Joseph Aiguier, 13402 Marseille, France, wirgin@1ma.cnrs-mrs.fr), Erick Ogam (Laboratoire de Mécanique et d'Acoustique CNRS UPR-7051, 31, Chemin Joseph Aiguier, 13402 Marseille Cedex 20, France, ogam@1ma.cnrs-mrs.fr), Laurent De Ryck (Lab. ATF, Katholieke Universiteit Leuven, Celestijnenlaan 200D, B-3001 Leuven, Belgium, Laurent.DeRyck@fys.kuleuven.be), Walter Lauriks (Lab. ATF, Katholieke Universiteit Leuven, Celestijnenlaan 200D, B-3001 Leuven, Belgium, Walter.Lauriks@fys.kuleuven.be), Claude Depollier (Laboratoire d'Acoustique de l'Université du Maine, Avenue Olivier Messiaen, 72085 Le Mans, France, claude.depollier@univ-lemans.fr)

The acoustic response (in particular, the transmission) of a periodic distribution of macroscopic inclusions within a rigid frame, porous plate (similar to a sonic crystal) is studied by the multipole method. Numerical results show that the addition of grating stacks leads to band gaps within the audible frequency range for a small number of stacks, this being associated with a large decrease of the transmission coefficient of the initial plate. The first band gap is of practical interest for noise suppression. The second band gap enables total acoustic absorption within a narrow frequency range due to the fact that a modified mode of the plate lies within this band gap.

\section{3:00}

1pPAb7. K-dependent polarization of sagittal acoustic waves in phononic crystals. Felipe Ramos-Mendieta (Universidad de Sonora, Rosales y Boulevard Luis Encinas, Colonia Centro, 83000 Hermosillo, Mexico, framos@cajeme.cifus.uson.mx), Betsabe Manzanares-Martinez (Division de Ciencias e Ingenieria, Unidad Sur Universidad de Sonora, Boulevard Lazaro Cardenas 100, 85390 Navojoa, Mexico, mbmm@cajeme.cifus.uson $. \mathrm{mx})$

By calculating the longitudinal and transverse strain energy averages we studied the polarization states of sagittal acoustic waves in one- and twodimensional phononic crystals. Our theoretical results show the continuous variation of the field displacement components when the Bloch wave vector sweeps the Brillouin zone; thus, the vibrational modes of a same dispersion curve can have different polarization. First we present the polarization map of sagittal waves for an epoxy/Sn superlattice. Then we discuss the band polarization of an array of cylindrical holes in epoxy. As we shall see, the mixed modes can be either predominantly transverse of predominantly longitudinal. For calculations we have used an energy balance criterion.

\section{3:20}

1pPAb8. Proof of the existence of large complete band gaps in high frequency silicon phononic crystal plates. Saeed Mohammadi (Georgia Institute of Technology, 338083 Georgia Tech Station, Atlanta, GA 30332, USA, saeedm@gatech.edu), Ali Asghar Eftekhar (Georgia Institute of Technology, 338083 Georgia Tech Station, Atlanta, GA 30332, USA, eftekhar@ece.gatech.edu), Abdelkrim Khelif (Institut FEMTO-ST/CNRS, 32 avenue de l'Observatoire, 25044 Besançon cedex, France, abdelkrim.khelif@femto-st.fr), William D. Hunt (Georgia Institute of Technology, 338083 Georgia Tech Station, Atlanta, GA 30332, USA, bill.hunt@ee.gatech.edu), Ali Adibi (Georgia Institute of Technology, 338083 Georgia Tech Station, Atlanta, GA 30332, USA, adibi@ece.gatech .edu)

We show, for the first time, the evidence of the existence of large phononic band gaps (PBGs) in two-dimensional phononic crystal (PC) plates formed by etching a hexagonal (honeycomb) array of air holes through a free standing plate of silicon (Si). A CMOS compatible fabrication process is used on a Si on insulator (SOI) substrate to realize the PC devices. More than $30 \mathrm{~dB}$ attenuation is observed for eight periods of the hexagonal lattice $\mathrm{PC}$ at high frequency region, i.e. $133 \mathrm{MHz}$ with a band gap to mid gap ratio of $25 \%$. We show that the experimental results agree very well 
with the theoretical predictions for the PBG. This result opens a new direction in the implementation of high frequency practical PC structures for a variety of applications especially wireless communication, and sensing.

\section{3:40}

1pPAb9. Acoustic defect mode in a finite 1-dimension lattice. Kanghyun Chu (Center for Noise and Vibration Control, Korea Advanced Institute of Science and Technology, 4114, Department of Mechanical Engineering, Guseong-dong, Yuseong-gu, 305-701 Daejon, Republic of Korea, kanghyunchu@kaist.ac.kr), Yang-Hann Kim (Center for Noise and Vibration Control, Korea Advanced Institute of Science and Technology, 4114, Department of Mechanical Engineering, Guseong-dong, Yuseong-gu, 305701 Daejon, Republic of Korea, yanghannkim@kaist.ac.kr)

If there is a defect in a periodic lattice, then the defect modes are expected. The wave localization can be one of representative examples of defect modes applications. However, it is not likely possible to deal with an infinite-periods lattice in practice, and this implies that it is impossible to obtain a perfect wave localization. This motivates us to study a lattice with a finite period which allows the wave tunneling through it. Therefore, the behavior of the acoustic wave in a 1-dimension periodic lattice containing a defect with a finite period has been studied. For simplicity, we considered the lattice composed of two kinds of media with different characteristic impedance in the repeating order with finite periods and the defect with arbitrary size and characteristic impedance Finally, the limitation of wave localization in the real world application is discussed.

\section{4:00-4:20 Break}

\begin{abstract}
$4: 20$
1pPAb10. Review of phononic crystals, nonlinear processes, devices and prospects. Sergey Nikitov (Institute of Radioengineering and Electronics, Russian Academy of Sciences, 11, bldg. 7, Mokhovaya St., 125009 Moscow, Russian Federation, nikitov@cplire.ru), Yurii Gulyaev (Institute of Radioengineering and Electronics, Russian Academy of Sciences, 11, bldg. 7, Mokhovaya St., 125009 Moscow, Russian Federation, nikitov@cplire.ru), Valery Grigorevsky (Institute of Radioengineering and Electronics, Russian Academy of Sciences, 11, bldg. 7, Mokhovaya St., 125009 Moscow, Russian Federation,vvig324@ms.ire.rssi.ru), Alexander Grigorevsky (Institute of Radioengineering and Electronics, Russian Academy of Sciences, 11, bldg. 7, Mokhovaya St., 125009 Moscow, Russian Federation, vig324@ms.ire.rssi.ru), Ivan Lisenkov (Institute of Radioengineering and Electronics, Russian Academy of Sciences, 11, bldg. 7, Mokhovaya St., 125009 Moscow, Russian Federation, lisenkov@cplire.ru), Roman Popov (Institute of Radioengineering and Electronics, Russian Academy of Sciences, 11, bldg. 7, Mokhovaya St., 125009 Moscow, Russian Federation, rspopov@gmail.com)
\end{abstract}

The review of current state of the art of bulk and surface linear and nonlinear acoustic wave propagation in phononic crystals $(\mathrm{PhC})$ is given. First, theoretical analysis of bulk acoustic waves propagation in 2D phononic crystals composed of elastic medium with periodic systems of air holes with different symmetry is considered. The properties of hypersonic bulk and surface acoustic waves (Love, Lamb) in $\mathrm{PhC}$ are considered theoretically and expermentally. We studied bulk waves propagation in microstructured optical fiber preforms made of quartz. The study of simultaneous propagation of acoustic waves and light in structures being both photonic and phononic crystals was done. We study also properties of guided waves (Lamb modes) propagating in layered structures containing the magnetic films with twodimensional periodic structures. In these structures we discovered a strong coupling between elastic and magnetic properties leading to effective waves transformation between magnetic and elastic systems. Such structures are so called magnonic-phononic crystals. The recommendations of use such unusual BAW and SAW properties in PhC for development of solid state devices are given. This work is supported by RFBR grant 08-02-00785.

\section{4:40}

1pPAb11. Omnidirectional phononic reflection and selective transmission in solid/fluid superlattices. El Houssaine El Boudouti (Faculté des Sciences, Département de Physique, Université Mohamed I,
60000 Oujda, Morocco, elboudouti@yahoo.fr),Youssef El Hassouani (Faculté des Sciences, Département de Physique, Université Mohamed I, 60000 Oujda, Morocco, hassouani@yahoo.fr), Hassan Aynaou (Faculté des Sciences, Département de Physique, Université Mohamed I, 60000 Oujda, Morocco, haynaou_2004@yahoo.fr), Bahram Djafari-Rouhani (IEMN, UMR CNRS 8520, avenue Poincaré, BP 60069, 59652 Villeneuve d'Ascq, France, Bahram.Djafari-Rouhani@univ-lille1.fr)

We present a theoretical analysis of the occurrence of omnidirectional reflection in one-dimensional phononic crystal. We discuss the conditions for a one-dimensional layered structure, made of alternating solid and fluid layers, to exhibit total reflection of acoustic incident waves in a given frequency range for all incident angles. In general, this property cannot be fulfilled with a simple finite superlattice if the incident wave is launched from an arbitrary fluid. Therefore, we propose two solutions to obtain such an omnidirectional band gap, namely: (i) cladding of the superlattice with a layer of high acoustic velocities that acts like a barrier for the propagation of phonons, or (ii) the association in tandem of two different superlattices in such a way that the superposition of their band structures exhibits an absolute acoustic band gap. We discuss the appropriate choices of the material and geometrical parameters to realize such structures. The behavior of the transmission coefficients is discussed in relation with the dispersion curves of the finite structure embedded between two fluids. By inserting a defect layer in the structure, we show that selective transmission may occur in the forbidden bands.

\section{5:00}

1pPAb12. Bubble phononic crystals. Alice Bretagne (Laboratoire Ondes et Acoustique, ESPCI, Université Paris 7, CNRS, 10 rue Vauquelin, 75005 Paris, France, alice.bretagne@espci.fr), Arnaud Tourin (Laboratoire Ondes et Acoustique, ESPCI, Université Paris 7, CNRS, 10 rue Vauquelin, 75005 Paris, France, arnaud.tourin@espci.fr), Patrick Tabeling (MMN ESPCI, 10 rue vauquelin, 75005 Paris, France, patrick.tabeling@espci.fr), Valentin Leroy (Laboratoire Ondes et Acoustique, ESPCI, Université Paris 7, CNRS, 10 rue Vauquelin, 75005 Paris, France,valeroy77@yahoo.fr), Nicolas Pannacci (MMN ESPCI, 10 rue vauquelin, 75005 Paris, France, nicolas.pannacci@espci.fr), Hervé Willaime (MMN ESPCI, 10 rue vauquelin, 75005 Paris, France, herve.willaime@espci.fr), Mathias Fink (Laboratoire Ondes et Acoustique, ESPCI, Université Paris 7, CNRS, 10 rue Vauquelin, 75005 Paris, France, mathias.fink@espci.fr)

Our aim is to investigate phononic crystals consisting of periodic arrangements of micron size gas bubbles. Such crystals are of great potential interest. Because of the high compressibility and high acoustic impedance contrasts between gas and water, a gas filled bubble in water is indeed a strong acoustic scatterer with a large value of its resonant wavelength compared with its size and a scattering cross section several orders of magnitude larger than its geometrical cross section. In that sense a microbubble can be viewed as the equivalent of an acoustic atom and even a small size sample made with such bubbles is expected to have a dramatic impact on acoustic propagation. Using microfluidics techniques we were able to produce perfectly monodisperse gas bubbles in a liquid. When these bubbles (radius $=50$ $\mu \mathrm{m})$ are then injected into a liquid with surfactant, they are found to organize themselves to form an hexagonal close packed crystal. Its stability has still to be optimised before testing it successfully with acoustic waves. The calculation of the band structure of that crystal with the plane wave expansion (PWE) method confirms its interest for controlling propagation of $\mathrm{MHz}-$ ultrasounds: a $800-\mathrm{kHz}$ wide band gap around $1.85 \mathrm{MHz}$ is predicted.

\section{$5: 20$}

1pPAb13. Floquet Lamb modes in periodic plates. Vincent Pagneux (Laboratoire d'Acoustique de l'Université du Maine, UMR CNRS 6613, AV. O. Messiaen, 72085 Le Mans, France, vincent.pagneux@univ-lemans.fr), Agnes Maurel (LOA/ESPCI, 10 rue Vauquelin, 75005 Paris, France, agnes.maurel@espci.fr)

An elastic waveguide with periodic perturbation of its thickness or of its elastic properties can support Floquet modes that are the extension of the classical Lamb modes. In this work, we obtain the spectra of the Floquet modes by using a new numerical coupled mode method which is based on a 
pseudospectral discretization in the transverse direction and a Magnus exponential integration in the longitudinal direction. The Floquet mode spectra are analyzed and compared to the Lamb mode spectra for different configuration of the periodic perturbation of the plate. A particular emphasis is given to the existence of backward wave propagation and to the number of propagating modes as the frequency is increased.

\section{$5: 40$}

1pPAb14. Acoustic transparency by switching of phononic band gaps. José Sanchez-Dehesa (Polytechnic University of Valencia, Cami de Vera s/n, $46022 \quad$ Valencia, Spain, jsdehesa@upvnet.upv.es), Helios Sanchis-Alepez (Polytechnic University of Valencia, Cami de Vera s/n, 46022 Valencia, Spain, hesana@alumni.uv), Daniel Torrent (Politechnic University of Valencia, Wave Phenomena Group. Department of Electronic Engineering, C/Camino Vera s.n, ES-46022 Valencia, Spain, datorma1@upvnet.upv.es), Liang-Wu Cai (Kansas State University, Mechanical and Nuclear Engineering, Manhattan, KS 66506, USA, cai@ksu .edu)

Acoustic transparency is here studied in two-dimensional phononic crystals made of hexagonal arrangements of layered cylinders. These layered cylinders, which have their acoustical properties radially dependent, can be achieved by using the recently proposed acoustical metamaterials [D. Torrent and J. Sanchez-Dehesa, New J. Phys., 9, 323 (2007)]. The transparency condition is here achieved by switching of the phononic band gaps, which is governed by the scattering form factor of the acoustic parameters (bulk modulus and sound speed). Various dependence have been studied. The feasibility of this proposal is demonstrated by multiple scattering simulations of the proposed metamaterials [Work supported by NSF of USA and MEC of Spain.]

\section{6:00}

1pPAb15. Three-dimensional phononic crystals made by brazing aluminum beads to form an opal structure. John H. Page (Dept. of Physics and Astronomy, Univ. of Manitoba, Winnipeg, MB R3T 2N2, Canada, jhpage@cc.umanitoba.ca), Hefei Hu (Dept. of Physics and Astronomy, Univ. of Manitoba, Winnipeg, MB R3T 2N2, Canada, hefeihu2@uiuc.edu), Yukihiro Tanaka (Division of Applied Physics, Graduate School of Engineering, Hokkaido University, 0608628 Sapporo, Japan, yuki@eng.hokudai.ac.jp), Takuro Okada (Department of Applied Physics, Hokkaido University, 060-8628 Sapporo, Japan, broaden_my_outlook@yahoo.co.jp), Shin Tamura (Department of Applied Physics, Hokkaido University, 060-8628 Sapporo, Japan, s-tamura@eng .hokudai.ac.jp)

Most phononic crystals studied so far are constructed from periodically arranged objects (e.g., spheres or rods) immersed in a continuous solid or fluid medium. In this presentation, we investigate the properties of a different type of phononic crystal made by weakly brazing 4-mm-diameter aluminum beads in a face-centred cubic array, producing a large-scale opal. In this crystal, wave propagation proceeds through the network of coupled beads rather than through the surrounding medium (air in our case), and the main band gaps that we observe result from coupled resonances of the beads rather than from Bragg scattering. Using ultrasonic techniques, we measure the transmission coefficient and the dispersion relation along the $\Gamma \mathrm{L}$ direction, and show that the lowest-frequency gap occurs between about 500 and
$600 \mathrm{kHz}$ - a frequency range that is just below the lowest resonance of an isolated aluminum sphere. We have also measured the group velocity, which we find to be negative in the lowest frequency band gap. Further insight into the properties of these phononic crystals is obtained by performing finite difference time domain (FDTD) calculations, in which the coupling between spheres is taken into account by allowing the spheres to overlap.

\section{6:20}

1pPAb16. Band gaps in a phononic crystal constituted by cylindrical dots on a homogeneous plate. Bahram Djafari-Rouhani (IEMN, UMR CNRS 8520, avenue Poincaré, BP 60069, 59652 Villeneuve d'Ascq, France, Bahram.Djafari-Rouhani@univ-lille1.fr), Yan Pennec (IEMN, UMR CNRS 8520, avenue Poincaré, BP 60069, 59652 Villeneuve d'Ascq, France, yan.pennec@univ-lille1.fr), Hocine Larabi (IEMN, UMR CNRS 8520, avenue Poincaré, BP 60069, 59652 Villeneuve d'Ascq, France, hocine.larabi@wanadoo.fr), Jérôme Vasseur (IEMN, UMR CNRS 8520, avenue Poincaré, BP 60069, 59652 Villeneuve d'Ascq, France, jerome.vasseur@univ-lille1.fr), Anne-Christine Hladky (IEMN, UMR CNRS 8520, avenue Poincaré, BP 60069, 59652 Villeneuve d'Ascq, France, anne-christine.hladky@isen.fr)

Using the finite difference time domain (FDTD) and the finite element (FE) methods, we investigate theoretically the band structure of a phononic crystal constituted by a periodic array of cylindrical dots deposited on a thin homogeneous plate. We demonstrate that such a structure can exhibit a very low frequency absolute band gap as compared to the wavelength in the constituent materials. The occurrence of this gap requires an appropriate choice of the geometrical parameters and, in particular, of the thickness of the plate as compared to the period or to the height of the cylinders. However, the same behavior can be obtained for various combinations of the materials constituting the homogeneous plate and the dots. We study in detail the width of this band gap as a function of the geometrical and physical parameters of the structure. On the other hand, the structure can also exhibit one or more higher gaps whose numbers can be increased by increasing the height of the cylinders.

\section{$6: 40$}

1pPAb17. Laser-generated surface acoustic waves in copper line arrays on silicon. Alexei A. Maznev (Department of Applied Physics, Hokkaido University, Kita 13 Nishi 8, Kita-ku, 060-8628 Sapporo, Japan, alexei .maznev@gmail.com)

Laser-generated acoustics waves are widely used to control the fabrication on metal interconnect structures in the semiconductor industry. On the other hand, acoustic properties of such structures, typically comprised of periodic arrays of metal lines embedded in a dielectric film on a silicon substrate, pose some interesting wave propagation problems. We will report measurements of surface acoustic waves (SAWs) performed using laserinduced transient grating technique on periodic arrays of micron-wide copper lines. As expected, SAW dispersion curves yield small band gaps at the Brillouin zone boundary. However, much larger band gaps are observed within the 1st Brillouin zone. We believe that these giant band gaps are formed due to avoided crossing of the 1st and 2nd order surface acoustic modes of the film/substrate structure when the 1st mode dispersion curve becomes folded due to periodicity. Another observed phenomenon is a spectacularly sharp increase in the acoustic attenuation occurring when the folded surface mode becomes coupled to a bulk wave. 


\title{
Session 1pPAc
}

\section{Physical Acoustics and Signal Processing in Acoustics: Acoustic Landmine Detection I}

\author{
James M. Sabatier, Cochair \\ University of Mississippi, NCPA, 1 Coliseum Drive, University, MS 38677, USA \\ Keith Attenborough, Cochair \\ Open University, Department of Design, Development, Materials and Environment, Walton Hall, Milton Keynes, MK7 6AA, UK
}

\section{Invited Papers}

\section{1:00}

1pPAc1. Combined seismic, radar, and induction sensor for landmine detection. Waymond R. Scott (Georgia Institute of Technology, School of Electrical and Computer Engineering, 777 Atlantic Dr., Atlanta, GA 30332-0250, USA, waymond.scott@ece.gatech.edu), Kangwook Kim (Dept. Machatronics, GIST, 1 Oryong-dong, Buk-gu, 500-712 Gwangju, Republic of Korea, mkkim@gist.ac.kr), Gregg D. Larson (Georgia Institute of Technology, G. W. Woodruff School of Mechanical Engineering, 777 Atlantic Dr., Atlanta, GA 30332-0250, USA, gregg.larson@me.gatech.edu), Ali C. Gurbuz (Georgia Institute of Technology, School of Electrical and Computer Engineering, 777 Atlantic Dr., Atlanta, GA 30332-0250, USA, gtg173p@mail.gatech.edu), James H. McClellan (Georgia Institute of Technology, School of Electrical and Computer Engineering, 777 Atlantic Dr., Atlanta, GA 303320250, USA, jim.mcclellan@ece.gatech.edu)

An experimental system to collect co-located ground penetrating radar (GPR), electromagnetic induction (EMI), and seismic data was developed to investigate possibilities of using the sensors in a cooperative manner and to investigate the benefits of the fusion of the sensors. These sensors were chosen because they can sense a wide range of physical properties. The seismic sensor is sensitive to the differences between the mechanical properties of a landmine and the soil while the GPR is sensitive to the dielectric properties, and the EMI sensor is sensitive to the conductivity. In the experiments, a range of mines and clutter objects were buried at various depths in a sandbox. Multiple burial scenarios were investigated with a variety of antipersonnel and antitank mines and typical clutter objects. The GPR makes use of modified resistive-vee antennas. The EMI sensor collects broadband data so that the relaxation spectra of the buried targets can be used to aid discrimination. The system uses electrodynamic shakers to generate seismic waves, and a radar is used to measure the displacement of the surface caused by the seismic waves. The responses of each of these sensors to the buried targets will be shown. [Work supported by ARO.]

\section{$1: 20$}

1pPAc2. Information-based sensor management for landmine detection using electromagnetic induction, ground-penetrating radar, and seismic sensors. Mark P. Kolba (Duke University, Department of Electrical and Computer Engineering, Box 90291, Durham, NC 27708, USA, mpk6@ee.duke.edu), Peter A. Torrione (Duke University, Department of Electrical and Computer Engineering, Box 90291, Durham, NC 27708, USA, pt@ee.duke.edu), Waymond R. Scott (Georgia Institute of Technology, School of Electrical and Computer Engineering, 777 Atlantic Dr., Atlanta, GA 30332-0250, USA, waymond.scott@ece.gatech.edu), Leslie M. Collins (Duke University, Department of Electrical and Computer Engineering, Box 90291, Durham, NC 27708, USA, lcollins@ee .duke.edu)

An information-based sensor management framework is presented that enables the automated tasking of a suite of sensors when detecting static targets. The sensor manager chooses the sensors to use and the grid-based locations to observe in order to maximize the expected information gain that will be obtained with each new sensor observation. Initially, sensor probabilities of detection and false alarm, Pd and Pf, are assumed to be known by the sensor manager. In a field setting, however, Pd and Pf cannot be known exactly, and so uncertainty modeling for $\mathrm{Pd}$ and $\mathrm{Pf}$ is also presented. The sensor manager is tested on real landmine data using electromagnetic induction (EMI), ground-penetrating radar (GPR), and seismic sensors. A matched subspace detector is used to process the EMI data, an adaptive pre-screening algorithm based on the least mean squares (LMS) adaptive filter is used to process the GPR data, and whitening followed by an energy detector is used to process the seismic data. The sensor manager is able to detect the landmines more quickly and more effectively than an unmanaged, blind-search approach. Using all three sensor modalities also results in superior detection performance to that achieved by only a single modality.

\section{$1: 40$}

1pPAc3. Nonlinear seismo-acoustic landmine detection. Dimitri Donskoy (Stevens Institute of Technology, Dabvidson Lab., 711 Hudson Street, Hoboken, NJ 07030, USA, ddonskoy@ stevens.edu)

The seismo-acoustic methods are among the most promising emerging techniques for the detection of landmines. Numerous field tests have demonstrated that buried landmines manifest themselves at the surface through resonance and nonlinear responses (or signatures) to acoustic/seismic excitation at the frequencies below $1000 \mathrm{~Hz}$. The resonance signatures are primarily due to mine's housing structural resonances. The nonlinear signatures explained by high contact nonlinearity at the mine-soil interface. These phenomena are utilized for landmine detection demonstrating high provability of detection and low false alarms. The paper provides overview of theoretical and experimental investigations conducted by the author and his colleagues at Stevens Institute of Technology. Among major 
accomplishments are discovery and explanation of mine's resonance behavior; soil/depth effect on buried mine's resonances; discovery and analysis of nonlinear acoustic interactions at the soil-mine interface; development of nonlinear quadratic and intermodulation detection algorithms based on dual-frequency excitation. Work supported by the U.S.Army NVESD, ARO and ONR.

1pPAc4. Hysteresis effects in nonlinear acoustic landmine detection. Murray S. Korman (Physics Dept., United States Naval Academy, Chauvenet Hall Room 295, 572 C Holloway Road, Annapolis, MD 21402, USA, korman@usna.edu)

In nonlinear acoustic landmine detection the vibration interaction of the top-plate of the buried plastic landmine with the adjacent soil is a subject of interest due to the strong nonlinear coupling. Using airborne sound excitation (in buried plastic anti-tank inert VS 1.6 and VS 2.2 landmine experiments) tuning curve behavior and generation of combination frequencies from two-tone tests were similar to the mesoscopic/nanoscale nonlinear elastic behavior observed in geomaterials like sandstone [J. Acoust. Soc. Am. 116, 3354-3369 (2004)]. Tuning curve measurements near resonance for increased acoustic amplitude (exhibiting softening) can be explained if hysteresis effects (characteristic of mesoscopic nonlinearity) are considered. The backbone curve (peak amplitude vs. corresponding resonant frequency from a family of tuning curves) exhibits mostly linear behavior for "off target" soil surface vibration measurements of a soil layer resonating over a rigid boundary. Backbone curves for "on target" measurements exhibit significantly more bending and curvature when a soil layer resonates over the compliant top-plate of the landmine. An oscillator with hysteresis modeled by a distribution of parallel spring elements each with a different threshold slip condition seems to describe the "off target" behavior, while a single bilinear hysteresis element describes the "on target" results. [Support by USNA.]

\section{$2: 20$}

1pPAc5. Accelerometer measurements of acoustic-to-seismic coupling above buried objects. Keith Attenborough (Open University, Department of Design, Development, Materials and Environment, Walton Hall, MK7 6AA Milton Keynes, UK, Keith .Attenborough@ioa.org.uk)

The surface velocity of sand inside a large PVC container, induced by the sound pressure from either a large loudspeaker radiating into an inverted cone and pipe or a B\&K point source loudspeaker mounted with its axis vertical, has been measured using accelerometers. Results of white noise and stepped frequency excitation are presented. Without any buried object the mass loading of an accelerometer creates resonances in the spectral ratio of sand surface velocity to incident acoustic pressure i.e. the acoustic-to-seismic (A/S) admittance spectra. The A/S responses above a buried compliant object are larger and distinctive. The linear A/S admittance spectra in the presence of a buried electronic components box have been studied as a function of burial depth and sand state. The nonlinear responses above the buried box have been studied as a function of depth, sand state and amplitude. Predictions of a modified 1D lumped parameter model have been found to be consistent with the observed nonlinear responses. Also the modified model has been used to explain features of the A/S responses observed when using an accelerometer without any buried object [Work supported by DSTL, UK.]

\section{2:40}

1pPAc6. Acoustic-laser land mine detection: Operationally enabling technologies. Robert Haupt (MIT Lincoln Laboratory, 244 Wood St., Lexington, MA 02421, USA, haupt@LL.mit.edu)

Reliable and cost effective methods are critically needed to locate the vast number of land mines left behind from military conflicts. Many mine location systems being developed fall short of being operationally practical since they may detect only metal or a specific mine feature, have limited standoff ranges, or have slow search speeds. An approach that may overcome operational issues is the acoustic-laser method. Acoustic waves generated by a noncontact/standoff source and transmitted through air can couple to the ground and induce resonances from a large variety of mines. The vibration field above these mines can be measured remotely using laser Doppler vibrometry. In this paper, enabling technologies that can significantly increase search area speed, improve standoff range, and reduce size in an acoustic-laser system are discussed. We take a novel approach to excite mines by using a high powered parametric acoustic array source developed at MIT Lincoln Laboratory that transmits a highly directive sound beam from a safe distance. The avalanche photo diode (APD) array developed at MIT Lincoln Laboratory will also be discussed as the enabling technology of a proposed 4096 multipixel laser system that can sense a wide vibration field out in front of a moving vehicle.

\section{3:00}

1pPAc7. Fast acoustic landmine detection using multiple beam laser Doppler vibrometry. Vyacheslav Aranchuk (University of Mississippi, NCPA, 1 Coliseum Drive, University, MS 38677, USA, aranchuk@olemiss.edu), James M. Sabatier (University of Mississippi, NCPA, 1 Coliseum Drive, University, MS 38677, USA, sabatier@olemiss.edu), Richard D. Burgett (Planning Systems, Inc., NCPA, 1 Coliseum Drive, University, MS 38677, USA, rburgett@olemiss.edu)

An acoustic method of buried landmine detection consisting of excitation of the ground using airborne sound or seismic waves in the frequency range from about $50 \mathrm{~Hz}$ to $1000 \mathrm{~Hz}$, and obtaining a velocity image of the ground surface with a laser Doppler vibrometer (LDV) has been developed and successfully tested in the laboratory and field experiments. The presence of a buried landmine can be detected by an abnormality in the velocity image. Initially, the scanning single-beam LDV created a velocity image of the ground through point-by-point measurements, which resulted in a long measurement time. To reduce this measurement time, a multiple beam LDV having 16 beams configured in a linear array was developed and successfully used in field experiments. To further reduce the time of measurement, a full-field LDV capable of making 256 vibration measurements in parallel on the target, configured as 16x16 square array of points equally separated in $\mathrm{x}$ - and $\mathrm{y}$-directions has been recently developed. The system is capable of creating a twodimensional vibrational velocity image of the ground surface in the time defined by the required frequency resolution. The parallel measurement makes the system a unique tool to measure transient vibrations. 
1pPAc8. Mechanical resonances in the low-frequency vibration spectrum of a cylindrically symmetric, anti-tank landmine. William C. Alberts (U.S. Army Research Laboratory, 2800 Powder Mill Rd, Adelhi, MD 21044, USA, kirk.alberts@arl.army.mil), James M. Sabatier (University of Mississippi, NCPA, 1 Coliseum Drive, University, MS 38677, USA, sabatier@olemiss.edu), Roger Waxler (University of Mississippi, NCPA, 1 Coliseum Drive, University, MS 38677, USA, rwax @olemiss.edu)

Acoustic-based landmine detection methods have enjoyed success, in part, because of structural resonances in many landmines. The unburied VS 1.6, a member of a family of plastic, cylindrically symmetric anti-tank landmines, exhibits seven modes below $1.6 \mathrm{kHz}$ and a large frequency shift of the first symmetric mode such that its frequency is greater than that of the first asymmetric mode, a phenomenon observed in timpani of reduced kettle volume [Christian et al., J. Acoust. Soc. Am., 76(5), 1336-1345 (1984)]. An elastically supported, thin, elastic plate acceptably models the unperturbed modes of the pressure plate. Coupling of the acoustic analog of the first symmetric mode to the cavities beneath the plate shows those cavities to be the cause of the perturbation. Shallow burial in sand effectively removes the frequency shift of the first symmetric mode. Comparisons to burial simulation experiments in water will be made, thus allowing a qualitative explanation of the effect sand has on the first symmetric mode of the plate. [This work was sponsored by US Army Research, Development, and Engineering Command, Night Vision and Electronic Sensors Directorate under Contract DAAB15-02-C-0024.]

3:40

1pPAc9. Review of a model for the response of a buried landmine to an airborne acoustic signal. Doru Velea (QinetiQ North America, Technology Solutions Group, 12030 Sunrise Valley Dr., Suite 400, Reston, VA 20191, USA, dvelea@ plansys.com), Roger Waxler (University of Mississippi, NCPA, 1 Coliseum Drive, University, MS 38677, USA, rwax@olemiss.edu), James M. Sabatier (University of Mississippi, NCPA, 1 Coliseum Drive, University, MS 38677, USA, sabatier@olemiss.edu)

Buried landmines can be found acoustically by insonifying the ground and detecting a contrast between the vibratory motion of the ground surface directly above the mine and a short distance away from the mine. Experiments have revealed a short-ranged spatial response and a frequency response that peaks around $100-200 \mathrm{~Hz}$ for anti-tank mines. The peak is believed to be a response to mechanical resonances of the mine itself. A model was developed to explain these experimental observations and to predict how the mine's resonance is influenced by the surrounding soil. In the model the soil was assumed to be an effective fluid while the mine was modeled as a right cylinder with a compliant top. The response of the top of the mine was modeled by an impedance condition. Predictions of the model are presented and compared with the phenomena observed in the field.

MONDAY AFTERNOON, 30 JUNE 2008

ROOM 352A, 4:00 TO 8:00 P.M.

\title{
Session 1pPAd
}

\section{Physical Acoustics: Sonic, Ultrasonic, and Megasonic Cleaning I}

\author{
Ralph T. Muehleisen, Cochair \\ Illinois Institute of Technology, Civil, Architectural and Environmental Engineering, 3201 S. Dearborn St., Room 228, \\ Chicago, IL 60616, USA \\ Claus-Dieter Ohl, Cochair \\ University of Twente, P.O. Box 217, Department of Science and Technology, Enschede, 7500 AE, Netherlands
}

\section{Invited Papers}

\begin{abstract}
4:00
1pPAd1. Global Trend of Precision Cleaning Technology and the Application in Hard Disk Drive Industry. Jun Zhang (Data Storage Institute, DSI bldg, 5 Eng Dr 1 (Off Kent Ridge Crescent, NUS), 117608 Singapore, Singapore, zhang_jun@dsi.a-star.edu.sg) , Yun Fook Liew (Data Storage Institute, DSI bldg, 5 Eng Dr 1 (Off Kent Ridge Crescent, NUS), 117608 Singapore, Singapore, zhang_jun@dsi.a-star.edu.sg)

As industry trends for hard disk drive and related electronic devices are moving toward high capacity and miniaturization. A big challenge is to find increasing the efficient ways to clean the parts which are regarded as the most stringent in cleanliness. The critical issues include the methodology for identifying the contaminants and how to choose a correct cleaning chemical and cleaning technology. Today's hard disk media, particularly the perpendicular recording media, is capable of storing up to 300GB per disk under which the distance between the recording head/disk have to be reduced to a few nanometers. It will bring more difficulties for increasing the reliability and disk capacities. On the other hand, all components within the hard disk drive are required to avoid submicron particulate contamination $(<0.1 \mu \mathrm{m})$. Megasonic and ultrasonic cleaning are the most effective and promising technologies to remove such contaminants. This presentation will discuss the technical trend and possible solution of precision cleaning and the strategy for selecting the cleaning chemicals and technologies to meet the cleanliness requirement through a case study.
\end{abstract}


1pPAd2. Accoustic cleaning in nanoelectronics. Paul Mertens (Imec, Kapeldreef 75, B-3001 Leuven, Belgium, paul.mertens @imec.be)

The fabrication of state-of-the-art electronic integrated circuits involves multiple cleaning steps in which residues of the order of ten or a few tens of $\mathrm{nm}$ need to be removed with very high efficiency. Historically cleaning was obtained by chemical undercutting (order of a few $\mathrm{nm}$ ). The dimensions of nanoelectronic devices become so small that the amount of substrate etching involved in a cleaning step should be kept below $0.05 \mathrm{~nm}$. Therefore other cleaning mechanism, involving a mechanical force, have to be considered such as acoustic agitation of the cleaning liquid or high-velocity aerosol bombardment. These forces, however, can damage fine structures already fabricated on the substrate, particularly the "up-features." This implies that a trade-off needs to be made between avoiding damage and obtaining an acceptable cleaning performance. This presentation includes typical hardware configurations and possible mechanisms for particle removal and use of megasonic agitation in nonaqueous liquid solutions.

\section{4:40}

1pPAd3. Cleaning of semiconductor substrates by controlled cavitation. Frank Holsteyns (SEZ AG, SEZ-Strasse 1, 9500 Villach, Austria, F.Holsteyns@at.sez.com), Alexander Lippert (SEZ AG, SEZ-Strasse 1, 9500 Villach, Austria, A.Lippert@at.sez.com), Florian Lechner (SEZ AG, SEZ-Strasse 1, 9500 Villach, Austria, F.Lechner@at.sez.com), Andrea Otto (Göttingen University, Friedrich-Hund-Platz 1, 37077 Göttingen, Germany, aotto@physik3.gwdg.de), Till Nowak (Göttingen University, Friedrich-Hund-Platz 1, 37077 Göttingen, Germany, tnowak@physik3.gwdg.de), Robert Mettin (Göttingen University, Drittes Physikalisches Institut, Friedrich-Hund-Platz 1, 37077 Göttingen, Germany, R.Mettin@physik3.gwdg.de), Alexander A. Doinikov (Belarus State University, 11 Bobruiskaya Street, 220030 Minsk, Belarus, doinikov@bsu.by), Harald Okorn-Schmidt (SEZ AG, SEZStrasse 1, 9500 Villach, Austria, H.Okorn-schmidt@at.sez.com)

The continuing downscaling of device geometries in the semiconductor industry is driving the requirements for both process and contamination control. Historically, the physical and the chemical processes required for contamination control were evolutionarily scaled with device geometry. However, today's tailored wet-chemical cleaning approaches must strive to meet stringent requirements to assure a minimal material loss and no damage to extremely fragile structures. While chemical solutions exist for the control of molecular-organic and metallic ion contamination, the physico-chemical solutions for the removal of nanosized particulate contamination to critical diameters below $20 \mathrm{~nm}$ are still undetermined. Therefore, the potential and the limitations of megasonic cleaning, which is mainly based on cavitation, are carefully balanced and a detailed understanding of the ongoing physical mechanisms is necessary to maintain a stable window of operation. The relevant active mechanisms present in such a cavitation driven cleaning process, will depend in great part on the characteristics of the applied sound field; that is to say on frequency, amplitude and uniformity and also on the properties of the cleaning liquid, i.e., the level of gasification, temperature, surface tension, and many others. Different techniques in conjunction with simulations and modeling are under development to improve the design of a novel cleaning tool that can address the challenges.

\section{5:00}

1pPAd4. Using megasonic agitation to extend chemical cleaning for nanotechnology device production. Cole Franklin (1241 East Dyer Road, Santa Ana, CA 92672, USA, colesfranklin@yahoo.com)

Aqueous and solvent chemicals are widely used in the semiconductor industry and as the technology nodes shrink device makers are working to extend these cleaning fluids to the next technology node. Internal data shows megasonic agitation can enhance polymer removal without complete dissolution for both aqueous and solvent chemicals. Data has shown solvents and semi-aqueous solutions show better selectivity with advanced material stacks over aqueous solution. It has been established that megasonic energy can enhance particle removal from semiconductor devices during cleaning processes. However, applied megasonic energy can also damage sensitive semiconductor devices during cleaning, especially in aqueous solutions. Aqueous fluids appear to promote much more damage for the same applied megasonic power than do most solvents. We show that some solvents have a higher threshold for cavitation than deionized water mixes. Since device manufacturers are working to extend their current cleaning chemicals to the 65 and $45 \mathrm{~nm}$ pilot production and research nodes, we studied several ways to accomplish this goal for aqueous or solvent chemicals. Described here we apply a methods to predict the damage of nanostructures by various liquids using signal analysis to better understand the mechanical that promotes improved control of cleaning without damage.

$$
5: 20
$$

1pPAd5. On frequency scaling of ultrasonic cavitation and cleaning. Robert Mettin (Göttingen University, Drittes Physikalisches Institut, Friedrich-Hund-Platz 1, 37077 Göttingen, Germany, R.Mettin@physik3.gwdg.de)

In virtually all applications of acoustic cavitation, variations of the effects with sound frequency are observed. However, a simple comparison of analogical setups at different frequencies can be misleading, as other parameters, like wavelength or emitted transducer power, change jointly with the driving frequency. Indeed, the similarity of acoustically cavitating systems under scaling of frequency is not well defined yet, in particular because it is not clear what observable should stay the same. A possible way of characterization of "similar" systems could be the observation of similar cavitation bubble structures, "such as" for hydrodynamic flow patterns or hydrodynamic cavitation structures. This idea is debated on experimental and theoretical basis, and the consequences for cleaning applications in the higher frequency range are discussed.

\section{$5: 40$}

1pPAd6. Surface cavitation on micro- and nanometer scales. Detlef Lohse (Physics of Fluids, University of Twente, P.O. Box 217, 7500 AE Enschede, Netherlands, d.lohse@utwente.nl), Nicolas Bremond (University of Twente, P.O. Box 217, Department of Science and Technology, 7500 AE Enschede, Netherlands, Nicolas.Bremond@espci.fr), Bram Borkent (University of Twente, P.O. 
Box 217, Department of Science and Technology, 7500 AE Enschede, Netherlands, b.m.borkent@tnw.utwente.nl),Claus-Dieter Ohl (University of Twente, P.O. Box 217, Department of Science and Technology, 7500 AE Enschede, Netherlands, c.d.ohl@utwente.nl), Manish Arora (University of Twente, P.O. Box 217, Department of Science and Technology, 7500 AE Enschede, Netherlands, m.arora@tnw.utwente.nl), Julius Vancso (University of Twente, P.O. Box 217, Department of Science and Technology, 7500 AE Enschede, Netherlands, g.j.vancso@tnw.utwente.nl), Holger Schoenherr (University of Twente, P.O. Box 217, Department of Science and Technology, 7500 AE Enschede, Netherlands, h.schonherr@utwente.nl)

Heterogeneous bubble nucleation at surfaces has been notorious because of its irreproducibility. Here controlled multibubble surface cavitation is achieved by heterogenous nucleation of bubbles on a hydrophobic surface patterned with microcavities. The expansion of the nuclei in the microcavities is triggered by an impulsive lowering of the liquid pressure. The procedure allows to control and fix the bubble-distance within the bubble cluster. We observe a perfect quantitative reproducibility of the cavitation events where the inner bubbles in the two-dimensional cluster are shielded by the outer ones, reflected by their later expansion and their delayed collapse. Apart from the final bubble collapse phase (when jetting flows directed towards the cluster's center develop), the bubble dynamics can be quantitatively described by an extended Rayleigh-Plesset equation, taking pressure modification through the surrounding bubbles into account. When repeating the same experiments with flat polyamide and hydrophobized silicon surfaces populated with surface nanobubbles (as can be seen through atomic force microscopy), these nanobubbles do not act as nucleation sites for cavitation bubbles, in contrast to the expectation. This implies that surface nanobubbles are not just stable under ambient conditions but also under enormous reduction of the liquid pressure down to $-6 \mathrm{MPa}$. We denote this feature as superstability.

\section{6:00}

1pPAd7. In-situ monitoring of megasonic cleaning. Aaldert G. Zijlstra (Physics of Fluids, University of Twente, P.O. Box 217, 7500 AE Enschede, Netherlands, a.g.zijlstra@tnw.utwente.nl), Tom Janssens (IMECvzw, Kapeldreef 75, 3001 Heverlee, Belgium, janssent@imec.be), Paul Mertens (Imec, Kapeldreef 75, B-3001 Leuven, Belgium, paul.mertens@imec.be), Michel Versluis (Physics of Fluids, University of Twente, P.O. Box 217, 7500 AE Enschede, Netherlands, m.versluis@utwente.nl)

Acoustic agitation is used in ultrasonic cleaning to induce a physical force to remove nanoparticles in semiconductor processing. The performance of cleaning tools is commonly quantified ex-situ by the particle removal efficiency $\eta$ (i.e. the ratio of the remaining to the original particle concentration). The resulting $\eta$-wafer maps often reveal spatial nonuniformities related to tool design. The local study of the cleaning dynamics, quantified by the removal frequency $\left(f_{R}\left[s^{-1}\right]\right)$ also indicates that the density of cleaning events over a wafer can be strongly non homogeneous. Because the sound frequencies used in industrial tools are in the $\mathrm{MHz}$ range, visualization of the cavitating bubbles (resonant size $3 \mu \mathrm{m}$ ) is highly challenging, both in time and in spatial resolution. Considering that the cleaning effect of a cavitating bubble is permanent a method is presented here to determine the acoustic cleaning event size $\left(A_{\text {event }}\left[\mu \mathrm{m}^{2}\right]\right)$ and the event flux $\left(\phi\left[\mu \mathrm{m}^{-2} \mathrm{~s}^{-1}\right]\right)$ through visualization techniques. The method consists of time-resolved imaging of the removal of a nanoparticle layer deposited on a transparent substrate. The microscopic cleaning activity can then be correlated to the actual removal frequencies $f_{R}$ through the relation $f_{R}=\phi A_{\text {event }}$ to further clarify the cleaning mechanisms in industrial tools.

\section{6:20}

1pPAd8. Potential uses of ultrasound in the dairy ultrafiltration processes. Shobha Muthukumaran (School of Engineering and Information Technology, Deakin University, Pigdons Road, Waurn Ponds, Victoria, 3217 Geelong, Australia, shobha.muthukumaran@deakin.edu.au), Sandra Kentish (Department of Chemical and Biomolecular Engineering, The University of Melbourne, Parkville, Victoria, 3010 Melbourne, Australia, sandraek@unimelb.edu.au), Muthupandian Ashokkumar (Department of Chemical and Biomolecular Engineering, The University of Melbourne, Parkville, Victoria, 3010 Melbourne, Australia, masho@unimelb.edu.au), Geoff Stevens (Department of Chemical and Biomolecular Engineering, The University of Melbourne, Parkville, Victoria, 3010 Melbourne, Australia, gstevens@unimelb.edu.au)

There has been a growing interest in the industrial application of ultrasound, especially in the food industry. Power ultrasound can have a number of physical effects; it can increase turbulence through both the introduction of vibrational energy and through acoustic streaming, it can cause both particle dispersion and particle agglomeration and clean surfaces with a scouring action. Our work in this area has focused on the use of ultrasound to enhance membrane processing. Low frequency ultrasound has been used to facilitate cross flow ultrafiltration of dairy whey solutions for both during the ultrafiltration production cycle and the cleaning cycle. During the production cycle, the use of ultrasound reduces both pore blockage and the specific resistance of the fouling cake layer. This leads to higher flux rates and the potential for longer production cycles. During the cleaning cycle, ultrasound systematically increases cleaning efficiency, thus has the potential to reduce both total chemical consumption and system downtime. There was no deterioration in cleaning effectiveness or membrane condition, which implies that sonication, has not damaged the membrane itself. Similarly, there was no change in the chemical nature of soluble proteins following sonication.

\section{6:40}

1pPAd9. Ultrasonic cleaning of particle and natural organic matter fouled cross-flow membranes. Linda K. Weavers (The Ohio State University, 470 Hitchcock Hall, 2070 Neil Ave, Columbus, OH 43210, USA, weavers.1@ osu.edu), Harold W. Walker (The Ohio State University, 470 Hitchcock Hall, 2070 Neil Ave, Columbus, OH 43210, USA, walker.455@osu.edu), Dong Chen (Lamar University, Department of Civil Engineering, Beaumont, TX 77710, USA, dchen@my.lamar.edu), Mikko O. Lamminen (The Ohio State University, 470 Hitchcock Hall, 2070 Neil Ave, Columbus, OH 43210, USA, m.o.lamminen@gmail.com)

Membrane filtration has arguably been the most significant development in the area of water purification in the past 50 years. It has enabled many processes to become more reliable and to achieve purities that have not been achieved previously. One of the drawbacks of membrane use is fouling. In this research the ultrasonic cleaning of particle-fouled membranes was investigated. Specifically, we have explored the mechanisms responsible for ultrasonic cleaning and how solution conditions, particle conditions, cake layer effects, 
and ultrasonic conditions affect cleaning using scanning electron microscopy (SEM) and permeate flux measurements. We have investigated continual cleaning during membrane filtration in addition to cleaning after fouling. Results of this work will be discussed for cross-flow system configurations.

\section{Contributed Papers}

\section{7:00}

1pPAd10. Measurements and simulations on the wall shear stress from single cavitation bubbles. Rory Dijkink (University of Twente, Physics of Fluids, Building Meander, Postbus 217, 7500 AE Enschede, Netherlands, r.j.dijkink@tnw.utwente.nl), Evert Klaseboer (Institute of High Performance Computing, 1 Science Park Road, \#01-01 The Capricorn, 117528 Singapore, Singapore, evert@ihpc.a-star.edu.sg), Boo Cheong Khoo (National University of Singapore, Dept of Mechanical Engineering, 10 Kent Ridge Crescent, 119260 Singapore, Singapore, mpekbc@nus.edu.sg), Claus-Dieter Ohl (University of Twente, P.O. Box 217, Department of Science and Technology, 7500 AE Enschede, Netherlands, c.d.ohl@utwente.nl)

Cavitation bubbles collapsing near a solid boundary manifest a jetting effect towards the closest rigid boundary. After impacting on the boundary the jet spreads out radially thereby shearing the surface. It is speculated that cavitation bubbles clean through the resulting shearing forces, e.g. they drag adherent contaminants through the radial spreading jet. Although the cleaning through bubbles is used in a wide set of technologies, for example for pre-cleaning surgical equipment, for bio-film removal in medical and drinking water applications, and in ultraclean processing of semiconductor wafers, very limited experimental studies on the wall shear stress exist. In an effort to shed light on the shear forces caused by no spherical oscillating cavitation bubbles close to boundaries we implement a constant temperature anemometer together with high-speed imaging to elucidate the fluid dynamics near the boundary. Additionally, we perform potential flow simulations with a boundary element method. The boundary layer is resolved with a convolution integral to the solution of Stokes' first problem. In general we find the generation of wall shear stress both in experiment and simulations during the growth and collapse of the bubble. Yet, very high shear stress is generated once the jet impacts and spreads on the boundary.

\section{$7: 20$}

1pPAd11. Cinephotographic observations of particle removal from a surface by acoustic cavitation. Lawrence A. Crum (Center for Industrial and Medical Ultrasound, Applied Physics Lab., University of Washington, 1013 NE 40th St., Seattle, WA 98105, USA, lac@apl.washington.edu), Michael R. Bailey (Center for Industrial and Medical Ultrasound, Applied Physics Lab., University of Washington, 1013 NE 40th St., Seattle, WA 98105, USA, bailey@apl.washington.edu), Michael S. Canney (Center for Industrial and Medical Ultrasound, Applied Physics Lab., University of Washington, 1013 NE 40th St., Seattle, WA 98105, USA, mcanney@u.washington.edu), Hong Chen (Center for Industrial and Medical Ultrasound, Applied Physics Lab. University of Washington, 1013 NE 40th St., Seattle, WA 98105, USA,
hopechen@u.washington.edu),Thomas Matula(Center for Industrial and Medical Ultrasound, Applied Physics Lab., University of Washington, 1013 NE 40th St., Seattle, WA 98105, USA, matula@apl.washington .edu), Christopher McInnes (Ultreo, Inc., 9461 Willows Road NE, Redmond, WA 98052, USA, chris@ultreo.com)

It is commonly believed that ultrasonic cleaners remove particles from a surface through acoustic cavitation, presumably in which a pulsating bubble interacts directly with the particle. We are unaware of any direct evidence of this interaction. Recently, however, we have used a high-speed movie camera to observe the removal of biofilm attached to a solid surface during exposure to a cavitation field. The biofilm consisted of Streptococcus mutans, a common oral bacterium, grown on a glass slide and observed under magnification to be both thinly coating the glass surface and clustered in larger colonies. The cavitation field was created by an Ultreo ${ }^{\mathrm{TM}}$ toothbrush which combines both vibrating bristles and an ultrasound transducer with waveguide, operating at a frequency of $324 \mathrm{kHz}$. When the waveguide was immersed in water containing bubbles from an ultrasound contrast agent $\left(\right.$ Optison $\left.{ }^{\mathrm{TM}}\right)$, visual observations could be made with the naked eye of biofilm removal. With high-speed cinephotography, it was possible to observe bacteria removal by the direct interaction of a cavitation bubble (cloud) and the colony. These observations will be presented along with our interpretations of the data. [Work supported in part by Ultreo $^{\mathrm{TM}}$, Inc.]

\section{7:40}

1pPAd12. Pressure fields and their effects in membrane cleaning applications. Fabian Reuter (Göttingen University, Drittes Physikalisches Institut, Friedrich-Hund-Platz 1, 37077 Göttingen, Germany, freuter@physik3.gwdg.de), Robert Mettin (Göttingen University, Drittes Physikalisches Institut, Friedrich-Hund-Platz 1, 37077 Göttingen, Germany, R.Mettin@physik3.gwdg.de), Werner Lauterborn (Göttingen University, Friedrich-Hund-Platz 1, 37077 Göttingen, Germany, W.Lauterborn @ physik3.gwdg.de)

It is known that the use of ultrasonic cavitation can support the cleaning of filtration membranes. We study the final purification step in drinking water production by ultrafiltration through a submerged filter module. The module consists of parallel polymer membrane sheets. Its filtration efficiency is rapidly decreased by fouling. Here, the removal of membrane attached fouling layers by backflushing can successfully be enhanced using ultrasound without membrane damage. To better understand the cleaning mechanism and cavitation properties for further optimization of efficiency, the sound pressure field is measured and the bubble distribution is characterized. Different sound field arrangements are investigated with respect to cleaning performance, while membrane integrity is controlled by turbidity and particle monitoring of the permeate. Results show that a strong influence due to the geometrical configuration can be present, for instance due to impedance effects of the membrane layers. 


\title{
Session 1pPAe
}

\section{Physical Acoustics: Mathematical and Numerical Methods I}

\author{
Andrew Piacsek, Cochair \\ Central Washington University, Department of Physics, 400 E. University Way, Ellensburg, WA 98926, USA \\ B. D. Zaitsev, Cochair \\ Institute of Radio Engineering and Electronics of RAS, Saratov Branch, Zelyonaya str., 38, 410019 Saratov, Russian Federation
}

\section{Contributed Papers}

7:00

1pPAe1. The peculiarities of energy characteristics of acoustic waves. Boris D. Zaitsev (Institute of Radio Engineering and Electronics of RAS, Saratov Branch, Zelyonaya str., 38, 410019 Saratov, Russian Federation, zai-boris@yandex.ru), Andrey A. Teplykh (Institute of Radio Engineering and Electronics of RAS, Saratov Branch, Zelyonaya str., 38, 410019 Saratov, Russian Federation, teplykhaa@mail.ru), Iren E. Kuznetsova (Institute of Radio Engineering and Electronics of RAS, Saratov Branch, Zelyonaya str., 38, 410019 Saratov, Russian Federation, kuziren@yandex.ru)

Acoustic waves propagating in piezocrystals transport kinetic and potential energies. Potential energy consists of mechanical, electrical, and mutual contributions. At that the change in potential energy of the voluentary unit of medium is identified with the work of mechanical stress for infinitesimal displacement. Versus method of integrating this equation there are two different expressions for density of potential energy. In case 1 (traditional approach) the density of energy is equal to the half-product of mechanical stress and strain. At that for any type of plane acoustic wave the instantaneous density of total energy in given point of medium depends on time and there is no mutual transformation of potential and kinetic energies. For this case there exists well-known traditional expression for power flow. In case 2 suggested by authors the density of potential energy is equal to half-product of mechanical stress gradient and displacement. At that the density of total energy does not depend on time. It means the mutual transformation of potential and kinetic energies according to motion equation. The new expression for power flow is derived for this case. The peculiarities of energy characteristics for piezoactive waves are discussed. The work is supported by RFBR grant $\mathrm{N}^{\circ} 08-08-00793$.

\section{$7: 20$}

1pPAe2. Audio acoustic modeling using full-wave methods. Timo Lahivaara (University of Kuopio, P.O. Box 1627, 70211 Kuopio, Finland, timo.lahivaara@uku.fi), Tomi Huttunen (University of Kuopio, P.O. Box
1627,70211 Kuopio, Finland, tomi.huttunen@uku.fi),Simo-Pekka Simonaho (University of Kuopio, P.O.Box 1627, 70211 Kuopio, Finland, simo-pekka.simonaho@uku.fi)

The numerical simulation of wave propagation poses a significant challenge in scientific computation. Historically, several approaches are explored in order to get a stable method that can be efficiently used for approximating wave propagation without excessive numerical dissipation or dispersion. Unfortunately, the traditional approaches, such as the finite element and the finite difference, require many discretization points per wavelength to obtain reliable solutions. In this study, two alternative full-wave methods for reducing the computational complexity are consider. The methods are the time-domain discontinuous Galerkin method and the ultraweak variational formulation in the frequency domain. Using these techniques, the directivity and the frequency response of a loudspeaker are studied. Moreover, the simulated results are compared to experimental measurements.

\section{7:40}

1pPAe3. Propagation in a dispersive medium: a phase-space approach. Leon Cohen (City University of New York, Hunter-Phyiscs, 695 Park Ave., New York, NY 10021, USA, leon.cohen@ hunter.cuny.edu)

We derive exact moments for pulse propagation in a dispersive medium. These moments are not only inherently interesting but clarify the validity of a recently proposed approximation scheme for wave propagation. The approximation method for pulse propagation is based on the Wigner positionwave/number representation and is very accurate, easy to apply, and moreover is physically illuminating. In particular one obtains the evolved approximate Wigner distribution from the initial Wigner distribution by a simple linear translation in phase space. Propagation with damping is also taken into account. We will show that the reason for the high accuracy of the approximation is that the important low order moments are exactly given by the approximation and that these low order moments preserve very well the basic shape of the pulse. Moreover, now that we understand why the approximation method works well, the approximation can be systematically improved. We give a number of specific examples of exactly calculable moments to illustrate the method and we compare exact and approximate moments. Research supported by the AFOSR. 


\title{
Session 1pPPa
}

\section{Psychological and Physiological Acoustics: Integrated Approaches to Auditory Scene Analysis I}

\author{
Mounya Elhilali, Cochair \\ Johns Hopkins University, Dept of Electrical and Computer Engineering, Barton Hall 105, 3400 N. Charles Street, \\ Baltimore, MD 21218, USA \\ Daniel Pressnitzer, Cochair \\ CNRS UMR 8158 ; Univ. Paris Descartes; Ecole Normale Supérieure, 29 rue d'Ulm, Paris, 75005, France
}

\section{Invited Papers}

1:00

1pPPa1. Sequential integration in the perception of tone sequences. Leon Van Noorden (Rassonstraat 39, B-1030 Brussels, Belgium, leonvannoorden@mac.com)

The seminal book of Albert Bregman on Auditory Scene Analysis had not yet appeared at the time of my dissertation research (1970-1975). One of the major difficulties that I had was to come at grips with the concept of a sequence of tones that form a 'gestalt' of tones 'belonging' together. This Sequential Integration, as coined by Bregman, has not received as much attention as the 'Differentiation' aspect. A number of experiments show that this sequential integration process is characterised by a resonance process with a broad peak near $2 \mathrm{~Hz}$. This resonance process can explain several phenomena such as subjective rhyhmisation, the data on tapping along polyrhythms and the histogram of musical tempi. Confronting these findings with the measurements of the Temporal Cohence Boundary may indicate where pitch movement detectors may play a role. Recent publications have linked this resonance with a movement control process.

$1: 20$

1pPPa2. On the binding of successive tones: Implicit versus explicit pitch comparisons. Laurent Demany (CNRS UMR 5227; Univ. Victor Segalen, 146 rue Léo Saignat, 33076 Bordeaux, France, laurent.demany@psyac.u-bordeaux2.fr), Daniel Pressnitzer (CNRS UMR 8158; Univ. Paris Descartes; Ecole Normale Supérieure, 29 rue d'Ulm, 75005 Paris, France, Daniel.Pressnitzer@ens.fr), Catherine Semal (CNRS UMR 5227; Univ. Victor Segalen, 146 rue Léo Saignat, 33076 Bordeaux, France, catherine.semal@u-bordeaux2.fr)

Listeners were presented with sound sequences in which one pure tone (T) was followed by a set (S) of five synchronous or asynchronous pure tones 550 cents apart. In a "present/absent" condition, T was either identical to a randomly selected component of S or halfway in frequency between two components, and listeners had to indicate if T was present in S or not. In an "up/down" condition, $\mathrm{T}$ was 100 cents below or above a randomly selected component of $\mathrm{S}$, and listeners had to identify the direction of the frequency shift. When the components of $\mathrm{S}$ were asynchronous, the present/absent task was easier than the up/down task. When the components of $\mathrm{S}$ were synchronous, the opposite trend was observed. In case of asynchrony, the components of $\mathrm{S}$ could be heard out individually, so listeners presumably compared explicit (conscious) pitch percepts to make their judgments. In case of synchrony, the components of $\mathrm{S}$ were difficult to hear out individually; apparently, pitch comparisons were then made implicitly by "frequency-shift detectors" (Demany and Ramos, 2005) which, we argue, participate in auditory scene analysis. It seems that such detectors relate automatically consecutive tones, and are generally less efficient for comparisons between nonconsecutive tones.

\section{$1: 40$}

1pPPa3. Concurrent sound segregation and peripheral frequency resolution. Christophe Micheyl (University of Minnesota, Department of Psychology, 75 E. River Road, Elliott Hall N218, Minneapolis, MN 55455, USA, cmicheyl@umn.edu), Michael V. Keebler (University of Minnesota, Department of Psychology, 75 E. River Road, Elliott Hall N218, Minneapolis, MN 55455, USA, keeb0002@umn.edu), Andrew J. Oxenham (University of Minnesota, Department of Psychology, 75 E. River Road, Elliott Hall N218, Minneapolis, MN 55455, USA, oxenham@umn.edu)

In everyday environments where multiple sound sources coexist, listeners often have to follow a harmonic source of interest (e.g., someone's voice) among other such sources (e.g., other talkers). Here, we review psychoacoustical findings suggesting that this form of auditory scene analysis is related to the degree of peripheral "resolvability" of harmonics in the auditory system. We present experimental results, which indicate a systematic relationship between harmonic resolvability and the ability of normal-hearing listeners to "track" (or discriminate) changes in the fundamental frequency (F0) of a target harmonic complex mixed with another (interferer). We show that poor harmonic resolvability is associated not only with poor performance, but also with an inability to take advantage of normally potent cues for concurrent sound segregation, including F0 differences, laterality differences, and onset-offset asynchronies. While it remains unclear whether this relationship is causal, these findings may have important implications for the design of artificial auditory-scene-analysis systems, and for our understanding of the listening difficulties experienced by hearing-impaired listeners in whom peripheral frequency resolution is reduced. [Work supported by NIDCD R01DC05216.] 
1pPPa4. Temporal fine structure cues in auditory stream segregation of complex tones. Nicolas Grimault (Laboratoire Neurosciences Sensorielles, Comportement, Cognition, UMR 5020, Université Lyon 1 - CNRS, 50 av. Tony Garnier, 69366 Lyon Cedex 07, France,ngrimault@olfac.univ-lyon1.fr), Etienne Gaudrain (Laboratoire Neurosciences Sensorielles, Comportement, Cognition, UMR 5020, Université Lyon 1 - CNRS, 50 av. Tony Garnier, 69366 Lyon Cedex 07, France, egaudrain@gmail.com)

The ability to understand speech-in-speech is generally described to be related to the capacity to segregate one auditory stream among others sound sources. The channeling theory suggests that sequential stream segregation is basically sustained by frequency selectivity. However, it has been evidenced that sequences of stimuli that evoked the same excitation pattern can be streamed apart based on temporal cues only. Other results involving hearing impaired listeners with enlarged auditory filters hardly fit in with this theory. More recently, several reports in the literature and new attempts of modelization underline the potential interest of temporal fine structure cues for stream segregation. The current study is dedicated to test further the potential interest of temporal fine structure cues to stream apart sequences of unresolved complex tones with alternating fundamental frequencies. Several conditions of envelope saliency (positive and negative Schroeder phase complex tones) and several conditions of fine structure will be used in a streaming test leading to an objective measure related to segregation.

2:20

1pPPa5. Auditory streaming and coherence. Shihab Shamma (ECE Department, AV Williams Bldg., 2203, University of Maryland, College Park, MD 20742, USA, sas@eng.umd.edu), Mounya Elhilali (Johns Hopkins University, Dept. of Electrical and Computer Engineering, Barton Hall 105, 3400 N. Charles Street, Baltimore, MD 21218, USA, mounyaelhilali@gmail.com)

Auditory streaming is a phenomenon that manifests itself in the everyday ability of humans and animals to parse complex acoustic information arising from multiple sound sources into meaningful auditory "streams." For instance, the ability to follow a conversation at a noisy cocktail party or hear the violin in the orchestra both rely on the formation of auditory streams. While seemingly effortless, the neural mechanisms underlying auditory streaming remain a mystery. In this talk, we shall discuss physiological experiments that address the role of coherence in mediating this percept. The experiments contrast the perception and neural responses evoked by the classic two-tone stimuli when presented in alternating or synchronous modes. Briefly, while the percepts in these two conditions are very different, being much more "streamed" in the alternating case, physiological responses to the tones did not differ significantly enough to explain this change in percept. This result casts doubt on the simple spatial segregation hypothesis, and instead argues for a substantial role of coherence of stimuli across different channels in mediating streaming.

\section{Contributed Paper}

\section{2:40}

1pPPa6. Auditory temporal edge detection in human auditory cortex. Maria Chait (UCL Ear Institute, 332 Gray's Inn Road, WC1X 8EE London, UK, m.chait@ucl.ac.uk)

Auditory objects are detected if they differ acoustically from the ongoing background. In simple cases, the appearance or disappearance of an object involves a transition in power, or frequency content, of the ongoing sound. However, it is more realistic that the background and object possess substantial nonstationary statistics, and the task is then to detect a transition in the pattern of ongoing statistics. How does the system detect and process such transitions? We use magnetoencephalography (MEG) to measure early auditory cortical responses to transitions between constant tones, regularly alternating, and randomly alternating tone-pip sequences, where the dimension of change is either frequency, loudness, or spatial location. Such transitions embody key characteristics of natural auditory temporal edges. Our data demonstrate that the temporal dynamics and response polarity of the neural temporal-edge-detection processes depend in specific ways on the generalized nature of the edge (the context preceding and following the transition) and suggest that distinct neural substrates in core and noncore auditory cortex are recruited depending on the kind of computation (discovery of a violation of regularity, vs the detection of a new regularity) required to extract the edge from the ongoing fluctuating input entering a listener's ears.

\section{Invited Papers}

1pPPa7. Perception of simultaneous sentences with frequency-shifted voiced excitation. Brian Roberts (Aston University, Psychology, Life and Health Sciences, B4 7ET Birmingham, UK, b.roberts@aston.ac.uk), Stephen D. Holmes (Aston University, Psychology, Life and Health Sciences, B4 7ET Birmingham, UK, S.D.Holmes@ aston.ac.uk), Chris J. Darwin (University of Sussex, Psychology, School of Life Sciences, BN1 9QG Brighton, UK, cjd@sussex.ac.uk)

Identification of key words in one of two simultaneous sentences is substantially improved when the sentences differ in fundamental frequency (F0). When the sentences are constructed to be almost continually voiced, identification can improve from $20 \%$ at 0 semitones difference to 80\% at 8 semitones difference (J. Bird \& C.J. Darwin 1998 in Psychophysical and Physiological Advances in Hearing, Ed A.R. Palmer et al., Whurr, London, pp. 263-269). We recorded the same monotone sentences as Bird and Darwin. These were first resynthesised using PSOLA to give a range of harmonic F0 differences $(0,1,3$, and 10 semitones) and then additionally resynthesised by LPC with the LPC residual frequency shifted by $25 \%$ of F0, to give excitation with inharmonic but regularly spaced components. Experiment 1 showed that frequency-shifted sentences gave the same large improvement with nominal F0 difference as did the harmonic sentences, although overall performance was about 10\% poorer. Experiment 2 replicated the finding by Bird and Darwin that swapping the F0 difference across spectral regions only reduced the improvement in recognition for large F0 differences, and extended this finding to frequency-shifted voiced excitation. The results extend the range of perceptual phenomena originally attributed to harmonic processing. 


\section{3:20-3:40 Break}

3:40

1pPPa8. Signal duration modulates age differences in neuromagnetic brain activity associated with concurrent sound segregation. Claude Alain (Rotman Research Institute, Baycrest Centre, 3560 Bathurst Street, Toronto, ON M6A 2E1, Canada, calain@rotman-baycrest.on.ca), Kelly McDonald (Rotman Research Institute, Baycrest Centre, 3560 Bathurst Street, Toronto, ON M6A 2E1, Canada, kmcdonald@ rotman-baycrest.on.ca)

Listeners' ability to parse concurrent sounds is a prerequisite in solving the cocktail party problem. Here, we examine whether deficits in periodicity coding can account for older adults' difficulties in understanding speech in noise. In two experiments, we measured auditory evoked fields (AEFs) while young and older adults listened to complex sounds that either had all of their harmonics in tune or had the third harmonic mistuned by 4 or $16 \%$ of its original value. For each participant, the AEFs were modeled with a pair of dipoles in auditory cortex and the effects of age on the resulting source waveforms were examined. Older adults were less likely to report hearing the mistuned harmonic as a separate sound than young adults, but only for short duration signals (50 ms). This age difference was paralleled by reduced neuromagnetic activity indexing the processing of the mistuned harmonic. For longer duration signals (e.g., $200 \mathrm{~ms}$ ), older adults show comparable amplitude, but a delay in latency. These findings show an age-related decline in concurrent sound perception based on harmonicity, which could partly be alleviated by increasing signal duration. The implications of these findings for speech perception in noise will be discussed.

\section{Contributed Paper}

4:00

1pPPa9. Influence of noise type, degree of comodulation and interaural phase difference on the combined monaural and binaural masking release. Bastian Epp (Universität Oldenburg, Medizinische Physik, Carlvon-Ossietzky Str. 9-11, 26111 Oldenburg, Germany, Bastian.Epp@uni-oldenburg.de), Jesko Verhey (Universität Oldenburg, Medizinische Physik, Carl-von-Ossietzky Str. 9-11, 26111 Oldenburg, Germany, jesko.verhey@uni-oldenburg.de)

Several masking experiments have shown that the auditory system is able to use coherent envelope fluctuations of the masker across frequency within one ear as well as differences in interaural disparity between signal and masker to enhance signal detection. The two effects associated with these abilities are comodulation masking release (CMR) and binaural mask- ing level difference (BMLD). The aim of the present study was to investigate the combination of CMR and BMLD. Thresholds of a sinusoidal signal were measured in a flanking band paradigm, i.e., in the presence of several narrowband noise maskers, (i) for two noise types (Gaussian and multiplied noise), (ii) various degrees of comodulation, and (iii) various interaural phase differences (IPD) of the signal. Thresholds decreased as the signal IPD increased and decreased as the masker comodulation increased. For both noise types, the maximum CMR was about $10 \mathrm{~dB}$ and the maximum BMLD was about $14 \mathrm{~dB}$. Thresholds where monaural and binaural cues were present showed an addition of the single effects, i.e., a maximum masking release of $24 \mathrm{~dB}$. A simplified model of the auditory system assuming a serial alignment of the across-frequency and the binaural processing stages is able to reproduce the dataset.

\section{Invited Papers}

1pPPa10. Speech in noise and the medial olivo-cochlear efferent system. Raymond Meddis (University of Essex, Department of Psychology, Wivenhoe Park, CO7 9SW Colchester, UK, rmeddis@essex.ac.uk), Robert Ferry (University of Essex, Department of Psychology, Wivenhoe Park, CO7 9SW Colchester, UK, rferry@essex.ac.uk), Guy J. Brown (University of Sheffield, Dept. of Computer Science, Regent Court, 211 Portobello Street, S1 4DP Sheffield, UK, g.brown@dcs.shef.ac.uk)

A central question in auditory scene analysis is how we are able to follow speech against a background of interfering noise. The question is particularly acute for artificial speech recognition algorithms and the hearing impaired. The medial efferent system has been suggested as one contributor to our ability to hear speech in noise. We have recently added efferent suppression to our model of the auditory periphery and evaluated it against physiological observations at the level of the basilar membrane and auditory nerve. We have also replicated a study using compound action potentials where a tone in a noise background became more salient when the efferent system was artificially activated. Visual representations of the computed auditory nerve response to speech in noise show considerable improvement when the efferent system is activated. Tests using the auditory model as a front end to a connected-word recognition algorithm also showed improved performance in the presence of noise when efferent effects were included. The benefits of efferent suppression include reduced compression and extension of the dynamic range of individual auditory nerve fibers.

\section{4:40}

1pPPa11. Contributions of the brainstem and below to auditory scene analysis. Ian M. Winter (Centre for the Neural Basis of Hearing, The Physiological Laboratory, Downing Street, CB2 3EG Cambridge, UK, imw1001@cam.ac.uk)

We are increasingly aware of the cues the auditory system uses in the segregation and fusion of auditory objects, however, we have very little evidence for how, or where, these cues are extracted in the auditory pathway. Here I will show how the inner ear and cochlear nucleus contribute to three classical auditory scene analysis phenomena. In the first, grouping by common onset; adaptation, lateral suppression, and recovery from suppression all play a role. In the second phemonenon, a sequential streaming task, commonly referred to as ABA, the contributing low-level mechanisms are frequency tuning and neural adaptation. Finally, in the third phenomenon, comodulation masking release, which describes the seemingly paradoxical situation whereby the detection of a masked signal is improved by the addition of coherently modulated energy remote from the signal frequency, lateral suppression is an important mediator. Taken together these studies suggest that neurophysiological processes at, or before, the level of the cochlear nucleus make an important contribution to auditory scene analysis. 
1pPPa12. The European starling as a model for understanding mechanisms underlying auditory scene analysis. Georg Klump (Oldenburg University, Zoophysiology \& Behavior Group, IBU, Fak 5, Carl von Ossietzky Str. 9-11, 26129 Oldenburg, Germany, georg .klump@uni-oldenburg.de)

The European starling is a songbird that has evolved mechanisms for the analysis of acoustic scenes exhibiting a number of similarities with human perception. Segregation of signals and background noise from different sources is enhanced due to the processing of differences in modulation patterns as is evident in experiments on the comodulation detection difference and and comodulation masking release. Similarly to auditory streaming in human subjects, integration of sequential signals into streams occurs in the European starling and it is affected by spectral and temporal properties of the sounds. The presentation reviews results from psychophysical experiments and compares these to observed neural response patterns of auditory forebrain neurons in starlings.

\section{$5: 20$}

1pPPa13. Identifying auditory events at a cocktail party: Principles of temporal integration and stream segregation. Elyse Sussman (Albert Einstein College of Medicine, Dept. of Neuroscience, 1410 Pelham Parkway S, Bronx, NY 10461, USA, esussman @aecom.yu.edu)

The question of how sequential sound elements are grouped into meaningful percepts has been within the focus of researchers for decades. This talk takes a new perspective by addressing how within-stream temporal integration occurs in the context of a cocktail party. In a noisy environment, the ability to identify specific events depends upon multiple processes: the overall segregation of sounds into streams as well as the within-stream integration of sequential sounds into meaningful units. This talk presents two studies that address the interaction of these two complex processes. Event-related potentials were used to determine effects of timing (Experiment 1) and of attention (Experiment 2) on the perception of within-stream sound events in multistream environments. Experiment 1 shows that within-stream temporal integration of sequential elements uses previously segregated streams as input. Experiment 2 shows that attention can override stimulus-driven processing biases to facilitate task demands. These results demonstrate different time courses for segregation of sounds into separate streams and for integration of sound elements into within-stream units. Thus, the findings suggest that different neural mechanisms interact in the perceptual organization of sequential sound elements.

\section{Contributed Papers}

\section{$5: 40$}

1pPPa14. Object formation versus object selection. Barbara Shinn-Cunningham (Boston University Hearing Research Center, 677 Beacon Street, Boston, MA 02215, USA, shinn@cns.bu.edu)

Auditory scene analysis is intricately linked to our ability to understand one acoustic source amidst competing sources. However, studies of selective attention often fail to distinguish between the roles of object formation (estimating the spectro-temporal content of a particular auditory object) and object selection (determining which object will be the focus of attention) in performance. Part of the difficulty in trying to disentangle these effects is that they are intimately related. However, listeners in a selective attention task often report sound elements that are similar in a sound dimension they know is task irrelevant when trying to selectively attend to a different sound attribute (e.g., location, pitch, intensity, timbre). Such results suggest a model of selective attention in which the focus of attention is an auditory object that is formed through interactions between automatic grouping processes and top-down selection. A conceptual model of these interactions will be presented along with data in support of this framework. [Work supported by AFOSR and ONR.]

\section{6:00}

1pPPa15. Auditory scene analysis emerges from a distributed yet integrated network. Rhodri Cusack (MRC CBU, 15 Chaucer Road,
Cambridge, CB2 7EF Cambridge, UK, rhodri.cusack@mrc-cbu .cam.ac.uk), Daniel Pressnitzer (CNRS UMR 8158; Univ. Paris Descartes ; Ecole Normale Supérieure, 29 rue d’Ulm, 75005 Paris, France, Daniel .Pressnitzer@ens.fr)

Three architectures that have been explicitly or implicitly proposed for auditory scene analysis were evaluated: (1) that some single module is responsible for scene analysis after feature extraction; (2) that scene structure is elaborated through feed-forward stages of auditory processing; and (3) that scene analysis is integrated yet distributed. It is argued that the available evidence points towards the third model. Against (1), scene analysis is influenced by features that are best represented across a broad range nodes in the auditory network, and transmitting this information to a single module would not be parsimonious. Furthermore, neurophysiology has shown a range of nodes reflect scene analysis, ranging from the cochlear nucleus, through auditory cortex to the parietal cortex. Against both (1) and (2) is the high degree of reciprocal interaction between the behavioural derivation of features and scene analysis, and the integration evident from the exclusivity of scene organisations. In contrast, model (3) naturally predicts these observations and is compatible with auditory anatomy, which is not "feed forward" or hublike. Finally, we highlight outstanding issues important to a fuller understanding of scene analysis, such as the neural code for perceptual groups and the mechanism for extraction of statistical regularities.

\section{Invited Papers}

\section{6:20}

1pPPa16. Perceptual dynamics of auditory streaming and its neural correlates. Makio Kashino (NTT Communication Science Laboratories, NTT Corporation, 3-1, Morinosato Wakamiya, 243-0198 Atsugi, Japan, kashino@avg.brl.ntt.co.jp), Hirohito M. Kondo (NTT Communication Science Laboratories, NTT Corporation, 3-1, Morinosato Wakamiya, 243-0198 Atsugi, Japan,hkondo@brl.ntt.co.jp), Minae Okada (ERATO Shimojo Implicit Brain Function Project, JST, 3-1, Morinosato Wakamiya, 2430198 Atsugi, Japan, mokada@ shimojo.jst.go.jp)

Prolonged listening to a repeated sequence consisting of low and high tones produces spontaneous transitions in the perceptual state between a single coherent stream and two segregated streams. The dissociation between constant physical stimulation and fluctuating perceptual experience in auditory streaming provides a compelling means for studying how auditory percepts are formed in the brain. First, we psychophysically examined the nature of such perceptual transitions in various frequency differences $(\Delta \mathrm{f})$ between low and 
high tones. After the initial buildup of streaming, perceptual transitions occurred frequently even at the $\Delta$ fs that were previously thought to produce a stable percept, and the dominant perceptual state changed depending on $\Delta \mathrm{f}$. Next, we explored brain activities correlated with the perceptual transitions using functional magnetic resonance imaging (fMRI). An event- related analysis revealed that the auditory cortex and thalamus were activated at the timing of perceptual transitions. The response onset of the activity in the auditory cortex was earlier than that in the thalamus for the transition from dominant to non-dominant percepts, and later for the transition from nondominant to dominant percepts. These results imply that the interaction of the auditory cortex and thalamus plays a crucial role for perceptual transitions in auditory streaming.

\section{6:40}

1pPPa17. What can binocular rivalry tell us about auditory streaming? Susan Denham (University of Plymouth, Drake Circus, PL4 8AA Plymouth, UK, sdenham@plymouth.ac.uk), István Winkler (Department of General Psychology, Institute for Psychology, Hungarian Academy of Sciences, P.O. Box 398, 1394 Budapest, Hungary, iwinkler@ cogpsyphy.hu), Kinga Gyimesi (Department of General Psychology, Institute for Psychology, Hungarian Academy of Sciences, P.O. Box 398, 1394 Budapest, Hungary, kgyimesi@cogpsyphy.hu), Gábor Stefanics (Department of General Psychology, Institute for Psychology, Hungarian Academy of Sciences, P.O. Box 398, 1394 Budapest, Hungary, gstefanics@ cogpsyphy.hu)

Although sensory systems share the common goal of building accurate representations of the environment, differences in the physical nature of stimuli from different modalities seem to argue against similar processing strategies. Nevertheless, our experiments have revealed surprisingly deep parallels between the dynamics of perceptual organisation in auditory streaming and binocular rivalry. These parallels provide evidence for an interpretation of auditory streaming in terms of rivalry between competing temporal associations, which helps to explain the distribution of perceptual switching with respect to stimulus features, strong differences between first and steady-state phases in ongoing switching, the nonmonotonic dependence of switching rate on stimulus "strength," the range of relationships between phase duration and stimulus features, and the existence and distribution of "transition" phases during which subjects simultaneously experience what are usually thought to be mutually exclusive perceptual states.

\section{7:00}

1pPPa18. Multistability in audiovisual speech scene analysis: behavioral and neurophysiological data on multimodal verbal transformations. Jean-Luc Schwartz (ICP-GIPSA, INPG, 46 Av. Félix Viallet, 38031 Grenoble, France, schwartz@icp.inpg.fr), Anahita Basirat (ICP-GIPSA, INPG, 46 Av. Félix Viallet, 38031 Grenoble, France, basirat@icp.inpg.fr), Marc Sato (ICP-GIPSA, INPG, 46 Av. Félix Viallet, 38031 Grenoble, France, sato@icp.inpg.fr)

The multistable perception of speech refers to the perceptual changes experienced while listening to a speech form cycled in rapid and continuous repetition, the so-called Verbal Transformation Effect. Because distinct interpretations of the same repeated stimulus alternate spontaneously, this effect provides an invaluable tool to examine how speech percepts are formed in the listener's mind. In a series of behavioural studies, we demonstrated that (1) articulatory-based representations play a key part in the endogenously driven emergence of auditory speech percepts; (2) vision penetrates into the transformation process, intervening both in the stability of a given pattern, and in the temporal dynamics of perceptual switches. Furthermore, we determined the cortical circuit in charge for the emergence of a shift of percept associated to a given audio input. This circuit involves a cortical "dorsal route" for speech perception, linking temporal, parietal, and frontal regions. Current experiments involve further tests about the nature of both perceptuo-motor and audiovisual interactions in verbal transformations. Altogether, these results shed some new lights on the cognitive and neurobiological bases of speech perception, in which multimodal interactions between sensory and motor representations play a crucial role. 


\title{
Session 1pPPb
}

\section{Psychological and Physiological Acoustics: Cochlear Implants: Going Beyond the Envelope I}

\author{
Bernhard U. Seeber, Cochair \\ MRC Institute of Hearing Research, Science Rd / University Park, Nottingham, NG7 2RD, UK \\ H. Steven Colburn, Cochair \\ Boston University, 44 Cummington St., Boston, MA 02215, USA
}

\section{Contributed Paper}

1:00

1pPPb1. A new approach to electric-acoustic stimulation. Christopher A. Brown (Arizona State University, Dept. of Speech \& Hearing Science, P.O. Box 870102, Tempe, AZ 85287-0102, USA, c-b@asu.edu), Sid P. Bacon (Arizona State University, Dept. of Speech \& Hearing Science, P.O. Box 870102, Tempe, AZ 85287-0102, USA, spb@asu.edu)

When low-frequency acoustic stimulation is combined with either real or simulated electric stimulation from a cochlear implant (electric-acoustic stimulation, or EAS), speech intelligibility in noise can improve dramatically. This improvement has been shown in simulation to be due in part to the presence of fundamental frequency (F0) and amplitude envelope information in the low-frequency region. The current experiment extends those findings to implant patients. Six patients who had residual lowfrequency hearing in either their implanted or unimplanted ear participated. A target talker was combined with multitalker babble and presented to the implant. In the low-frequency region, patients heard either no stimulus, target speech, or a tone that was modulated in frequency to track the dynamic changes in F0, and in amplitude with the amplitude envelope of the low-pass target speech. Results showed that the tone provided, on average, about 58 percentage points of improvement over electric-only stimulation. Both the tone and target speech provided a statistically significant benefit over electric stimulation only $(\mathrm{p}<0.0001)$, and were statistically equivalent to each other $(\mathrm{p}>0.05)$. These results demonstrate that a tone that conveys F0 and amplitude envelope information can provide significant benefit in EAS.

\section{Invited Papers}

1pPPb2. Temporal pitch processing by cochlear implant users. Robert P. Carlyon (MRC CBU, 15 Chaucer Rd., CB2 7EF Cambridge, UK, bob.carlyon@mrc-cbu.cam.ac.uk), Ying-Yee Kong (Notheastern University, 106A Forsyth, Boston, MA Ma 02115, USA, yingyeekong@googlemail.com), Cathy Lynch (Addenbrooke's NHS Trust, Hills Rd, CB2 2QQ Cambridge, UK, catherine.lynch@addenbrookes.nhs.uk), John Deeks (MRC CBU, 15 Chaucer Rd., CB2 7EF Cambridge, UK, john.deeks@mrc-cbu .cam.ac.uk)

Modern cochlear implants (CIs) convey fundamental frequency (F0) information using a purely temporal code. However, temporal pitch processing - as measured by the discrimination of the changes in the rate of a pulse train applied to a single electrode - is often worse than in normal hearing $(\mathrm{NH})$ at low pulse rates, and usually deteriorates dramatically at rates above about 300 pps. We will describe evidence that at low pulse rates, the value of the pitch perceived can be affected by refractory effects at the level of the auditory nerve $(\mathrm{AN})$, and can also be influenced by small $(<0.5 \mathrm{~dB})$ changes in stimulus level. In contrast, the deterioration in rate discrimination at high rates appears to be impervious to a wide range of manipulations that would be expected to strongly affect the representation of the stimulus at the level of the AN. One example of this is the finding that the variation in rate discrimination performance with baseline rate for single-pulse-per-period pulse trains correlates, across listeners, with discrimination of different rates of sinusoidal amplitude modulation imposed on a 5000-pps carrier. The implications of these findings for attempts to improve pitch coding in CIs will be discussed.

\section{$1: 40$}

1pPPb3. Investigating the influence of pulse rate and duration on pitch perception in cochlear implants. Joshua S. Stohl (Duke University, Department of Electrical and Computer Engineering, Box 90291, Durham, NC 27708, USA, jss@ee.duke.edu), Chandra S. Throckmorton (Duke University, Department of Electrical and Computer Engineering, Box 90291, Durham, NC 27708, USA, cst@ee.duke.edu), Leslie M. Collins (Duke University, Department of Electrical and Computer Engineering, Box 90291, Durham, NC 27708, USA, lcollins@ee.duke.edu)

Multirate sound processing strategies may potentially provide improved speech recognition and music perception in cochlear implants (Nie et al., 2005; Throckmorton et al., 2006), although it may be necessary to tune these strategies using psychophysical data to provide maximum benefit to the user (Throckmorton et al., 2006). To investigate the need for tuning, cochlear implant users participated in several rate-based psychophysical experiments. Subjects performed single- and two-rate pitch ranking tasks to investigate the influence of pulse rate on the overall pitch structure. The introduction of a second rate resulted in frequent pitch reversals between closely spaced electrodes. In addition to the multi-rate pitch structure, the duration necessary to perceive a rate change must also be considered. Experiments were performed using ABA stimuli, where rate B was higher than rate A. Subjects completed an embedded rate discrimination task in which the duration of B was fixed and its rate varied adaptively for durations ranging from 20-200 ms. The rate of segment B was then fixed, and its duration varied adaptively. Results imply that embedded rate difference limens may be a function of duration, and that the minimum duration required for detecting a change in pulse rate is subject specific. 
1pPPb4. Use of simultaneous stimulation to represent fine structure in cochlear implant processors. Leonid Litvak (Advanced Bionics, 12740 San Fernando Rd., Sylmar, CA 91342, USA, LeonidL@ AdvancedBionics.Com), Aniket Saoji (Advanced Bionics, 12740 San Fernando Rd., Sylmar, CA 91342, USA, Aniket.Saoji@AdvancedBionics.Com), Anthony Spahr (Arizona State University, Lattie F. Coor Hall, Room 3462, Tempe, AZ 85287, USA, Tony.Spahr@Asu.Edu), Abhijit Kulkarni (Advanced Bionics, 12740 San Fernando Rd., Sylmar, CA 91342, USA, Abhijit.Kulkarni@AdvancedBionics.Com), Michael Marzalek (Advanced Bionics, 12740 San Fernando Rd., Sylmar, CA 91342, USA, mikemarz@sonic.net)

In cochlear implants, simultaneous stimulation of adjacent electrodes, can be used to shape the electric fields generated within the cochlea. For example, simultaneous in-phase stimulation of nearby electrodes can be used to create activation patterns which may have maxima at locations that are intermediate to the locations of the stimulated electrodes. Psychophysically, such "virtual channels" give rise to intermediate pitch sensations. Simultaneous out-of-phase stimulation of two flanking electrodes in addition to the center electrode can be used to presumably produce more focused activation at the cost of larger overall stimulation currents. Forward masking patterns indicate that for sufficiently large compensation currents, such "focused" stimulation can lead to a more spatially contained excitation. In some cases, current focusing also could lead to sensations that are more tonal relative to monopolar. This presentation will review recent results obtained in our laboratories with both "virtual channel" and "focused" configurations. We will also address how both techniques can be used to better represent the within-channel fine structure in a wearable sound processing strategy.

1pPPb5. Effect of channel interactions on sensitivity to binaural timing cues in electrical hearing. Gary Jones (University of Wisconsin, 1500 Highland Ave, Waisman Center, Madison, WI 53705, USA, gjones@waisman.wisc.edu), Ruth Litovsky (University of Wisconsin, 1500 Highland Ave, Waisman Center, Madison, WI 53705, USA, litovsky@waisman.wisc.edu), Richard Van Hoesel (CRC HEAR, 384-388 Albert St, VIC 3002 East Melbourne, Australia, rvanh@bionicear.org)

With bilateral cochlear implant (BICI) users it is possible to test sensitivity to interaural timing differences (ITDs) while bypassing peripheral auditory processing. Ongoing work in our lab suggests that at low pulse rates ITD JNDs in the tens of microseconds can be achieved by BICI users with postlingual onset of deafness at single pairs of electrodes at the base, middle or apex of the electrode array. The current project is concerned with channel interactions and, specifically, performance when there is activation of more than one electrode pair and when more than one ITD is presented to the auditory system simultaneously. We examined ITD sensitivity in BICI users with bilateral pairs of pitch-matched electrodes on which unmodulated pulse trains were presented at 100 pulses per second. A probe pulse train and an added pulse train were temporally interleaved. ITD JNDs for the probe train were measured while varying the added train's: a) frequency place, b) level, and c) ITD (matched to probe or fixed at $0 \mu \mathrm{s}$ ). Results suggest that stimulation of multiple electrodes can result in a range of interaction effects that can have either facilitatory or interfering effects on binaural sensitivity. Work supported by NIH-NIDCD (R01 DC003083-09, F31 DC009361).

\section{2:40}

1pPPb6. Binaural jitter improves sensitivity to interaural time differences in electric and acoustic hearing. Bernhard Laback (Austrian Academy of Science/Acoustics Research Institute, Wohllebengasse 12-14, 1040 Vienna, Austria, Bernhard.Laback@oeaw.ac.at), Piotr Majdak (Austrian Academy of Science/Acoustics Research Institute, Wohllebengasse 12-14, 1040 Vienna, Austria, piotr@majdak.com), Matthew J. Goupell (Austrian Academy of Science/Acoustics Research Institute, Wohllebengasse 12-14, 1040 Vienna, Austria, matt.goupell@gmail.com)

Cochlear implant (CI) listeners are often sensitive to fine structure ITD at low pulse rates, but their sensitivity declines at higher pulse rates which are required for speech coding. We hypothesized that this limitation is related to the binaural adaptation phenomenon [Hafter and Dye, J. Acoust. Soc. Am. 73 644-651 (1983)], associated with periodic stimulation. With five CI listeners, we tested the effect of introducing binaurally-synchronized jitter (binaural jitter) in the stimulation timing, assuming that this reduces the periodicity in the neural response and thus avoids binaural adaptation. Pulse rates from 400 to 1515 pulses per second (pps) were tested. In addition, we performed a similar experiment with normal-hearing $(\mathrm{NH})$ listeners using bandpass-filtered acoustic pulse trains and testing the pulse rates 600 and 1200 pps. The CI listeners showed large improvements in ITD sensitivity from binaural jitter at high pulse rates (800 1515 pps). The NH listeners also showed large improvements from binaural jitter. The results are consistent with the hypothesis that random temporal variation reactivates the adapted binaural auditory system. Thus, binaurally-jittered stimulation improves the access of bilateral CI listeners to ITD information. Partly supported by the Austrian Science Fund, FWF, project number P18401-B15.

\section{3:00}

1pPPb7. Parameters affecting the precedence-effect with cochlear implants. Bernhard U. Seeber (MRC Institute of Hearing Research, Science Rd., / University Park, NG7 2RD Nottingham, UK, seeber@ihr.mrc.ac.uk), Ervin R. Hafter (University of California, Department of Psychology, 3210 Tolman Hall, Berkeley, CA, CA 94720-1650, USA, hafter@berkeley.edu)

Monaural spectral and temporal cues as well as binaural cues provide the information to analyze the auditory scene. Subjects using bilateral cochlear implants (CIs) have limited access to those cues, particularly to interaural time differences (ITDs) and to spectral information. Nevertheless, many subjects can localize sounds in quiet based on the evaluation of interaural level differences (ILDs) but this might be impaired by the presence of reflections. We studied the precedence effect with patients wearing bilateral CIs and found that localization was abolished in most subjects when the echo was present, but some subjects showed dominance of the leading sound on localization. The reasons for the different outcomes were studied with simulations of CIs. Using a noise-band vocoder simulation, subjects could not fuse lead and lag into a single object and they localized both separately. With a sinusoidal vocoder, lead-lag fusion and the precedence effect could be obtained provided that frequencies of the carrier sinusoids were matched across ears. Interaural phase of the carrier played only a limited role. We conclude that precedence of ongoing sounds can solely be based on ILDs and envelopeITDs and that proper place matching of CI-electrodes would help the analysis of concurrent sounds. 


\section{3:20-3:40 Break}

3:40

1pPPb8. Neural cross-correlation metrics to quantify envelope and fine-structure coding in auditory-nerve responses. Michael Heinz (Purdue University, 500 Oval Drive, West Lafayette, IN 47907, USA, mheinz@purdue.edu), Jayaganesh Swaminathan (Purdue University, 500 Oval Drive, West Lafayette, IN 47907, USA, jswamy@purdue.edu)

Fundamental questions about the relative perceptual importance of envelope and fine structure are often addressed using specialized acoustic stimuli, such as temporal-fine-structure speech or auditory chimaeras. Interpretation of these perceptual studies assumes envelope and fine structure can be isolated at the output of the cochlea. Narrowband cochlear filtering constrains the ability to isolate fine structure from envelope; however, envelope recovery from fine structure has been difficult to evaluate physiologically. Separate neural cross-correlation coefficients (CCCs) for envelope and fine-structure were developed based on shuffled auto- and cross-correlograms. Neural CCCs have a wide dynamic range for both within-fiber, cross-stimulus and cross-fiber, within-stimulus correlations based on both model and recorded spike-train data from auditory-nerve fibers. Results provide physiological evidence consistent with perceptual findings that envelope recovery is reduced as the number of analysis bands increases, but is not completely eliminated for 8- and 16band conditions. Neural CCCs were also used to evaluate across-fiber temporal coding, which has been implicated in the difficulties that hearing-impaired listeners have in understanding speech in complex acoustic backgrounds. Results demonstrate the potential of these neural CCC metrics to quantitatively evaluate a wide range of perceptually significant temporal coding issues relevant to normal and impaired hearing. Supported by NIH-NIDCD.

\section{Contributed Paper}

\section{4:00}

1pPPb9. Transmission of temporal fine structure by a penetrating auditory nerve implant. John C. Middlebrooks (University of Michican, KHRI, 1301 E. Ann St., Ann Arbor, MI 48109-5506, USA, jmidd@umich.edu), Russell L. Snyder (University of California, Epstein Laboratory, Room U-490, P.O. Box 0526, San Francisco, CA 941430526 , USA, rsnyder@ohns.ucsf.edu)

We are quantifying the transmission of temporal fine structure from a penetrating auditory nerve electrode array to the inferior colliculus (IC) in anesthetized cats. We stimulate with biphasic electrical pulse trains and determine the maximum pulse rate eliciting phase-locked activity. Many IC neurons showed significant phase locking to rates as high as 600 pulses per second ( $\mathrm{pps}$ ) when the nerve was stimulated with the penetrating array, whereas phase locking reached only 300 pps when stimulated with a conventional intrascalar cochlear implant. Phase locking to rates $>300$ pps was found primarily among IC units with characteristic frequencies $(\mathrm{CFs})<2$ $\mathrm{kHz}$. Such low frequency fibers are not stimulated selectively by conventional intrascalar cochlear implant. We selectively stimulated apical fibers by placing ball electrodes on the apical spiral lamina. Activation of these balls selectively stimulated low-CF IC neurons. These neurons phase locked to 600 pps. Based on these results, we conclude that transmission of temporal information from a penetrating intraneural electrode array is superior to that obtained with a conventional cochlear implant but only insofar as the intraneural electrodes provide more selective access to low-CF pathways. Supported by NIDCD NO1-DC-5-0005.

\section{Invited Papers}

1pPPb10. Why is sensitivity to interaural time differences (ITD) poorer than normal with bilateral cochlear implants? Neurophysiological considerations. Bertrand Delgutte (Massachusetts Eye \& Ear Infirmary, Eaton-Peabody Lab., 243 Charles St., Boston, MA 02114, USA, Bertrand_Delgutte@meei.harvard.edu), Kenneth E. Hancock (Massachusetts Eye \& Ear Infirmary, EatonPeabody Lab., 243 Charles St., Boston, MA 02114, USA, ken_hancock@MEEI.HARVARD.EDU)

Bilateral cochlear implantation improves sound localization and speech reception in noise. Yet ITD sensitivity is clearly poorer than normal with bilateral implants, even when the stimulation bypasses the processors (which discard the temporal fine structure of the stimulus). This poor behavioral ITD sensitivity is surprising from a physiological perspective because (1) electric stimulation produces precise temporal patterns of activity in the auditory nerve, and (2) most neurons in the inferior colliculus of acutely-deafened, bilaterally-implanted cats show precise tuning to ITD of pulse trains at the appropriate intensity (Smith and Delgutte, J. Neurosci. 27:6740). We will present physiological and modeling results bearing upon two hypotheses for reconciling neural and psychophysical observations. One hypothesis is that plastic changes to the neural circuitry resulting from binaural deprivation may impair the binaural processing in long-term deafened animals (and humans), particularly if deafening occurs during the neonatal period. Alternatively, the abnormal spatio-temporal pattern of activity across the population of binaural neurons (due in part to limited dynamic range with electric stimulation) may impair the ability of the central decoding stages to extract the ITD information available in individual binaural neurons.

\section{4:40}

1pPPb11. Modeling responses of brainstem neurons to electrical stimuli. H Steven Colburn (44 Cummington St., Boston, MA 02215, USA, colburn@bu.edu), Yoojin Chung (44 Cummington St., Boston, MA 02215, USA, yjchung@bu.edu), Andrew Brughera (44 Cummington St., Boston, MA 02215, USA, brughera@bu.edu), Yi Zhou (Johns Hopkins University, Dept. of Biomedical Eng.; School of Medicine, Ross 424 Wang, Baltimore, MD 21205, USA, zhouyi@jhu.edu)

This talk describes modeling efforts to understand brainstem neural responses to electrical cochlear stimulation. Our approach is to combine brainstem models developed for acoustic stimulation with descriptions of auditory-nerve (AN) responses to electric stimulation. Predictions for the behavior of neurons at several levels of the brainstem are compared to available physiological data. Specifically, the AN model predicts differences in discharge probability, degree of phase-locking, and adaptation of discharge rate of the AN response to stimuli, inlcuding both electrical and acoustical cases. Predicted neural responses are generated for several brainstem 
nuclei, including cell populations in the cochlear nucleus (CN), the superior olive (SOC) and the inferior colliculus (IC). Specific attention is given to two special topics: 1) responses to the simultaneous stimulation of multiple electrodes, and 2) the effects of amplitude modulation on the tuning of binaural neurons to interaural time delay (ITD). [Work supported by US National Institutes of Health grants DC04663, DC00100, and DC05775 (Delgutte, PI).]

\section{Contributed Papers}

\section{5:00}

1pPPb12. Higher sensitivity of human auditory nerve fibers to positive electrical currents. Olivier Macherey (MRC CBU, 15 Chaucer Rd., CB2 7EF Cambridge, UK, olivier.macherey@mrc-cbu.cam.ac.uk), Robert P. Carlyon (MRC CBU, 15 Chaucer Rd., CB2 7EF Cambridge, UK, bob.carlyon@mrc-cbu.cam.ac.uk), Astrid Van Wieringen (ExpORL, Dept. Neurosciences, K.U. Leuven, Herestraat 49 bus 721, O. \& N2, B-3000 Leuven, Belgium, astrid.vanwieringen@med.kuleuven.be), John Deeks (MRC CBU, 15 Chaucer Rd., CB2 7EF Cambridge, UK, john.deeks@mrc-cbu.cam.ac.uk), Jan Wouters (ExpORL, Dept. Neurosciences, K.U. Leuven, Herestraat 49 bus 721, O. \& N2, B-3000 Leuven, Belgium, jan.wouters@med.kuleuven.be)

Most contemporary cochlear implants (CIs) stimulate the auditory nerve with trains of amplitude-modulated, symmetric biphasic pulses. Although both polarities of a pulse can depolarize the nerve fibers and generate action potentials, it remains unknown which of the two (positive or negative) phases has the stronger effect. Animal experiments have shown that cathodic (negative) current flows are more effective than anodic (positive) ones in eliciting neural responses, and this finding has motivated the development of novel speech-processing algorithms. Here we show electrophysiologically and psychophysically that the human auditory system exhibits the opposite pattern, being more sensitive to anodic stimulation. We measured electrically evoked compound action potentials in CI listeners for phase-separated pulses, allowing us to tease out the responses to each of the two oppositepolarity phases. At an equal stimulus level, the anodic phase yielded the larger response. This finding was corroborated by a measure of psychophysical masking patterns and may relate to a particular orientation of the nerve fibers relative to the electrode or to a substantial degeneration/demyelination of the peripheral processes. Potential applications to improve $\mathrm{CI}$ speech-processing strategies are discussed.

\section{$5: 20$}

1pPPb13. 3D-localization of virtual sound sources in normal-hearing and cochlear-implant listeners. Piotr Majdak (Austrian Academy of Science/Acoustics Research Institute, Wohllebengasse 12-14, 1040 Vienna, Austria, piotr@majdak.com), Bernhard Laback (Austrian Academy of Science/Acoustics Research Institute, Wohllebengasse 12-14, 1040 Vienna, Austria, Bernhard.Laback@oeaw.ac.at), Matthew J. Goupell (Austrian Academy of Science/Acoustics Research Institute, Wohllebengasse 12-14, 1040 Vienna, Austria, matt.goupell@gmail.com)

The ability to localize sound sources in 3D-space was tested in humans Five normal-hearing (NH) subjects listened via headphones to noises filtered with subject-specific head-related transfer functions. Four bilateral cochlear implant (CI) subjects listened via their clinical speech processors to noises filtered with subject-specific behind-the-ear head-related transfer functions. A virtual structured environment was presented via head mounted display. Two conditions were used: a condition where the subjects were naive and had no response feedback and a learning condition where the subjects were trained by providing extensive feedback during the test. Response feedback was provided via the visual virtual environment. The results show that the CI listeners performed generally worse than NH listeners, both in the horizontal and vertical dimensions. Both subject groups were able to learn to better localize sound sources, which is supported by lower localization errors in the learning condition. However, in the learning condition, the CI listeners showed a front/back confusion rate comparable to naive $\mathrm{NH}$ listeners, which was two times higher than for the trained NH listeners. These results indicate the necessity of new CI processing strategies which include spectral localization cues. Funding by FWF (P18401-B15).

\section{$5: 40$}

1pPPb14. Emergence of sound localization acuity in children who are fitted with bilateral cochlear implants. Ruth Litovsky (University of Wisconsin, 1500 Highland Ave, Waisman Center, Madison, WI 53705, USA, litovsky@waisman.wisc.edu), Tina Grieco-Calub (University of Wisconsin, 1500 Highland Ave, Waisman Center, Madison, WI 53705, USA, grieco@waisman.wisc.edu), Shelly Godar (University of Wisconsin, 1500 Highland Ave, Waisman Center, Madison, WI 53705, USA, godar @ waisman.wisc.edu)

Sound localization was investigated in children who are born deaf and hear with either monaural or bilateral cochlear implants (CIs). Using a 2AFC discrimination task with stimuli to the right/left, in a sound proof booth, loudspeaker locations varied along the horizontal plane between $+/-70$ deg. Minimum audible angle (MAA) thresholds were estimated adaptively. In study 1, 4-14 year-old children who received two CIs in sequential procedures participated. They were first tested while functioning monaurally, then at 3-and 12-months following activation of bilateral hearing. Following bilateral activation, MAA thresholds were significantly lower, especially after 12 months. In study 2 children were 2.5 years old, with 6-20 months of bilateral experience. MAAs were typically better when children had $\geqslant 12$ months of exposure to bilateral stimulation. Overall, these findings suggest that binaural hearing abilities can be established in an auditory system that is strictly stimulated with electrically pulsed signals, but that unlike a normal auditory system, here a year of exposure may be required before localization acuity is better under bilateral than monaural conditions. These findings are especially relevant to considerations of localization in absence of fine-structure, a cue that is discarded by the speech processors utilized in CIs. 


\title{
Session 1pSAa
}

\section{Structural Acoustics and Vibration and EURONOISE: Vibration and Radiation from Complex Structural Systems I}

\author{
David Feit, Cochair \\ Applied Physical Sciences Corp., Ste. 300, 2 State St., New London, CT 06320, USA \\ Jean-Louis Guyader, Cochair \\ INSA de Lyon - LVA, Bâtiment St. Exupéry, 25 bis avenue Jean Capelle, Villeurbanne Cedex, F-69621, France
}

\section{Invited Papers}

1:00

1pSAa1. Development of a general SEA subsystem formulation using FE periodic structure theory. Vincent Cotoni (ESI Group, 12555 High Bluff Dr., Suite 250, San Diego, CA 92130, USA, vincent.cotoni @esi-group-na.com), Phil S. Shorter (ESI Group, 12555 High Bluff Dr., Suite 250, San Diego, CA 92130, USA, pjs@esi-group-na.com), Robin S. Langley (University of Cambridge, Trumpington Street, CB5 8HU Cambridge, UK, rs121@ hermes.cam.ac.uk)

Statistical Energy Analysis (SEA) represents a field of study in which statistical descriptions of a system are employed in order to simplify the analysis of complicated vibro-acoustic problems. In SEA, a vibro-acoustic system is represented by a collection of subsystems that can receive, store, dissipate and transmit vibro-acoustic energy. Traditionally, the SEA parameters for a given subsystem are formulated analytically based on consideration of wave propagation through the subsystem. While such analytical algorithms can be readily applied to the majority of systems encountered in practical problems, there are certain types of sections that are difficult to describe using existing analytical formulations. Examples include: isogrid in launch vehicle fairings, extruded aluminum sections in train floors and modern corrugated aircraft fuselage constructions. This paper describes the development of a generic SEA subsystem formulation based on the use of finite element (FE) periodic structure theory. A small unit cell of the section is created and computationally efficient algorithms are developed to calculate wave propagation through a large array of such cells. The resulting algorithms are used to calculate the SEA parameters for the section. The approach is described and a number of numerical validation examples are presented.

$1: 20$

1pSAa2. Structure/cavity coupling using Statistical Energy Analysis: Coupling loss factors and energy maps into subsystems. Nicolas Totaro (INSA de Lyon - LVA, Bâtiment St. Exupéry, 25 bis avenue Jean Capelle, F-69621 Villeurbanne Cedex, France, nicolas.totaro@insa-lyon.fr), Jean-Louis Guyader (INSA de Lyon - LVA, Bâtiment St. Exupéry, 25 bis avenue Jean Capelle, F-69621 Villeurbanne Cedex, France, jean-louis.guyader@insa-lyon.fr)

Prediction of interior noise is one of the most concerning issues of vehicle industry. Statistical Energy Analysis theoretically allows to determine energy spread over a structure divided into subsystems when one subsystem is submitted to a rain-on-the-roof excitation. Subsystems can be either a part of the structure or a cavity. Recently, a method (SmEdA) based on modal bases of uncoupled subsystems have been derived. This method permits to compute CLF using standard FEM software. This method has been successfully applied on structure/structure coupling and is extended to estimation of CLF between a structure and a cavity in the present article. In addition, in the case of interior noise, SEA can only provide a global energy into the cavity on frequency bands. No information on energy repartition into the subsystem is given. In the present article, an extension of SmEdA method is proposed to quickly estimate energy repartition into subsystems (structure or cavity).

\section{$1: 40$}

1pSAa3. Theory and application of pseudo-damping in structures. Adnan Akay (Carnegie Mellon University, Mechanical Engineering Department, Pittsburgh, PA 15213, USA, akay@cmu.edu), Antonio Carcaterra (Università di Roma, 'La Sapienza,' Dipartimento di Meccanica ed Aeronautica, Via Eudossiana, 18, 00184 Roma, Italy, a.carcaterra@dma.ing.uniroma1.it)

This presentation describes the underlying physics and theory of pseudo damping to reduce vibration of conservative structures. The theoretical approach is based on particular properties of harmonic functions that lead to construction of a complex system that exhibits nearly irreversible energy transfer. This approach also provides the ability to tailor energy absorption from a particular structure in the absence of damping. The authors will also discuss extension of the concept to continuous systems and its application to waveguides. The presentation will illustrate test results including application to reduce vibrations in a satellite. [Research carried out while AA served at NSF.] 
1pSAa4. Localization and mesoscopics in structures and rooms I. Richard Weaver (Dept. of Physics, University of Illinois, Urbana, IL 61801, USA, r-weaver@uiuc.edu)

Diffuse field methods such as reverberation room acoustics and statistical energy analysis predict wave energy flow in complex structures. They are predicated on an incorrect assumption of incoherence. The consequent failures of otherwise successful theories are occasionally striking. The most noteworthy among these are related to Anderson - and other kinds of - localization. Residual coherence manifests also in time-reversal invariance and Green's function retrieval in field-field correlations. It is sometimes possible in special cases to make ad hoc corrections to the diffuse field theories. It would be preferable, however, to augment them with more a fundamental quantitative theory applicable to a wide variety of systems. This talk reviews mesoscopic phenomena such as weak Anderson localization, modal echoes, Anderson localization, Thouless localization, and Greens function retrieval. It sets out the phenomena and concepts behind the new theory to be presented in "Localization and Mesoscopics in Structures and Rooms II" at a later session.

1pSAa5. Spherical harmonic analysis of the field radiated by a source embedded in a two-layer coating of a spherical shell. Jerry H. Ginsberg (Georgia Insitute of Technology, G. W. Woodruff School of Mechanical Engineering, Atlanta, GA 30332-0405, USA, jerry.ginsberg@me.gatech.edu)

The acoustic field generated by a small source embedded in a viscoelastic layer encapsulating a spherical shell was modeled previously by treating each layer as a dissipative fluid [J. H. Ginsberg, JASA, 122, 3067 (2007)]. Using spherical harmonic series for each layer was shown to lead to profoundly ill-conditioned equations because of the enormous differences of scale of the spherical Bessel functions having complex argument and high order. Mie series is not directly applicable because the present source is embedded in a layer. Scaling of the spherical Bessel functions and the alternatives of using Bessel and Neumann or two Hankel functions are examined for their efficacy in addressing ill-conditioning. The specfic system that is analyzed consists of a soft inner layer that coats the shell, covered by an outer layer that encapsulates the source. The impedance of the outer layer nominally matches the surrounding water, so reflection of the source from the soft inner layer might seem to have the effect of creating a dipole. However, depending on the wavelength in the inner layer, it might seem that the source "sees" the shell, in which case one would expect the behavior to be like a simple source whose strength is doubled.

\section{Contributed Papers}

\section{2:40}

1pSAa6. Periodic assembly of multicoupled beams: Wave propagation and natural modes. Guillaume Gosse (Laboratoire Vibrations Acoustique - INSA Lyon, 25 bis avenue Jean Capelle, Bâtiment Saint-Exupéry, F-69621 Villeurbanne cedex, France, guillaume.gosse@insa-lyon.fr), Charles Pezerat (Laboratoire Vibrations Acoustique - INSA Lyon, 25 bis avenue Jean Capelle, Bâtiment Saint-Exupéry, F-69621 Villeurbanne cedex, France, charles.pezerat@insa-lyon.fr), François Bessac (CETIAT, Domaine Scientifique de la Doua, 25 avenue des Arts, BP 2042, F-69603 Villeurbanne cedex, France, francois.bessac@cetiat.fr)

The present work is concerned with the vibrations of a discrete multicoupled periodic system. It lies within a larger study on the vibroacoustic behaviour of a heat exchanger. These structures are usually made of a succession of huge number of identical parallel fins (around 600 per meter) connected by tubes conveying the coolant fluid. By now their behaviour can not be calculated using FE model. As a first step, the periodicity principles are applied on a simpler structure, i.e.. an assembly of identical beams linked by several damped springs. The basic unit is symmetric and composed of one flexural beam with several springs on each side. Using the Floquet-Bloch's theorem and the works of Denys Mead (receptance matrix), it is possible to completely describe the whole structure behaviour (natural modes, response) only from the vibroacoustic knowledge of the basic unit. This has be done analytically and the results were confirmed by a (very time consuming) FE model calculation. The study of the basic unit can also give valuable information on the physical phenomena governing the transmission from one unit to the next, and then the propagation in the whole structure.

\section{3:00}

1pSAa7. The response of a complex dynamical system to a complex external forcing. John McCoy (Catholic University, 620 Michigan Ave, Washington, DC 20064, USA, mccoy@ cua.edu)

The response of a dominant grounded spring/mass to which are attached a large number of significantly less massive sprung masses, when subject to an external forcing acting on the dominant mass, is revisited. Distinguishing the new study is a focus on the net force that the attached systems cause to act on the dominant mass and the derivation of a semi-analytic, time domain representation of the system operator that transforms the history of the ex- ternal force acting on the adorned dominant spring/mass to the history of an effective force that acts on the unadorned dominant spring/mass. The mathematical framework provided by the concept of an "effective force" acting on the unadorned simple system gives additional insight to a commonly accepted concept of an "effective dissipation" modifying the unadorned simple system, when subject to simple external force histories; i.e., force histories with limited time extents or with limited bands of spectral content. Of greater practical interest is the mathematical framework allows investigation of external force histories that are not simple.

\section{3:20}

1pSAa8. General absorbing boundary for acoustic and elastic waves. Denis Duhamel (ENPC, UR Navier, 6 et 8 Avenue Blaise Pascal, Cité Descartes, Champs sur Marne, 77455 Marne la Vallée, France, duhamel@lami .enpc.fr)

In the finite element computation of unbounded acoustic problems, the domains must be of finite size and artificial absorbing conditions have to be introduced in order to avoid reflections at the truncated boundary. This communication proposes a new method in the frequency domain to generate efficient absorbing boundary conditions without the need to consider high order derivatives on the boundary. Moreover the approach is quite general and can consider media with mean flow or be applied to structural vibrations. It needs the knowledge of the dynamic stiffness matrix of only one element which can be obtained from any finite element software. The final result is an impedance condition linking the forces and displacements on the boundary which is determined numerically from a wave analysis of one element of the media. Using this impedance condition a finite element problem can be solved by standard methods. This is then applied to study some examples for 2D standard acoustic, acoustic problems with mean flow and vibrations of plates.

\section{3:40}

1pSA9. Dynamic response of an insonified sonar window. Andrew J. Hull (Naval Undersea Warfare Center, Code 8212, 1176 Howell St., Newport, RI 02841, USA, hullaj@npt.nuwc.navy.mil)

This talk derives and evaluates an analytical model of an insonified sonar window in contact with an array of Tonpilz transducers operating in 
receive mode. The window is fully elastic so that all wave components are present in the analysis. The output of the model is a transfer function of a transducer element output voltage divided by input pressure versus arrival angle and frequency. This model is intended for analysis of sonar systems that are to be built or modified for broadband processing. The model is validated at low frequency with a comparison to a previously derived thin plate model. Once this is done, an example problem is studied so that the effects of higher order wave interaction with acoustic reception can be understood. It was found that these higher order waves cause multiple nulls in the region where the array detects acoustic energy and that their locations in the arrival angle-frequency plane can be determined. The effects of these nulls in the beam patterns of the array are demonstrated.

\section{4:00-4:20 Break}

\section{4:20}

1pSAa10. Circumferential admittance approach for vibro-acoustic prediction of a submerged cylindrical shell with rib aperiodicity and transversal bulkheads. Laurent Maxit (DGA (Defence Agency), CTSN/SDP/CMPF, B.P. 28, 83800 Toulon, France, laurent.maxit@dga .defense.gouv.fr)

The circumferential admittance approach is presented to predict the vibro-acoustic behaviour of an externally fluid loaded shell with aperiodic stiffeners and transversal bulkheads. It consists to consider the circumferential spectral displacements of the shell and to use a partitioning of the structure such that the fluid loaded shell constitutes one subsystem and the stiffeners/bulkheads constitute others subsystems. Each subsystem is characterised independently by the circumferential admittances. For the stiffeners/bulkheads, a standard FE code is used to calculate these admittances with a shell description. The admittances of the fluid load shell are estimated by a spectral approach. Developments are proposed in this paper to improve the numerical convergence of the spectral approach. The final assembly is obtained using the continuity relations. Excitations like mechanical forces, acoustic diffuse fields or plane waves can be easily taken into account. For submarine applications, this model permits to study the noise radiated by the pressure hull and to analyse the acoustic target strength of the shell with its internal frames. The whole submarine pressure hull can be described with the assumption that it is extended by a cylindrical rigid baffle. Numerical validations are presented and results at several $\mathrm{kHz}$ are shown for a submarine application.

\section{4:40}

1pSAa11. Enhanced coupled mechanical-acoustic field computations on nonmatching grids. Simon Triebenbacher (Univ. ErlangenNuremberg, Dept. of Sensor Technology, Paul-Gordan-Str. 3/5, 91052 Erlangen, Germany, simon.triebenbacher@1se.eei.uni-erlangen.de), Manfred Kaltenbacher (Univ. Erlangen-Nuremberg, Dept. of Sensor Technology, Paul-Gordan-Str. 3/5, $91052 \quad$ Erlangen, Germany, manfred.kaltenbacher@1se.eei.uni-erlangen.de), Martin Meiler (Simetris $\mathrm{GmbH}$, Am Weichselgarten 7, 91058 Erlangen, Germany, martin.meiler@simetris.de), Hermann Landes (Simetris GmbH, Am Weichselgarten 7,91058 Erlangen, Germany, hermann.landes@simetris.de)

In this work we extend our research on nonmatching finite element grids, as used in the analysis of coupled mechanical-acoustic systems, from standard (bi-)linear grids to higher order Lagrangian grids. In practical applications, such as the simulation of thin membranes, the use of higher order finite elements is often advantageous to prevent locking effects. For the comparison of the radiated sound from a loudspeaker with measurements an acoustic propagation domain is required which is very large in relation to the size of the speaker. To keep the number of unknowns in this domain low the discretization should be chosen as coarse as possible without becoming susceptible to numerical dispersion. Due to the same reason it is also desirable to use a uniform discretization and to be able to adjust the approximation order. To gain more flexibility, we introduce a nonmatching interface between the subdomains and discretize them independently, both in space and approximation order. Our enhanced scheme applies the mortar finite element method to coupled mechanical-acoustic field problems. We demonstrate the applicability of our approach to practical applications. We analyze the system response of an electrodynamic loudspeaker and the sound radiation of an electric transformer.

\section{5:00}

1pSAa12. Comparison of numerical techniques for the vibro-acoustic behaviour of a flexible structure. Yvon Goth (CETIM, 52 av Felix Louat, BP 80067, F-60304 Senlis Cedex, France, yvon.goth@cetim.fr)

The tractors bonnets are large flexible parts that radiate noise under the excitation of the forces exerted on the contact points and the sound pressure fluctuations coming from the engine. The vibro-acoustic behaviour of such a bonnet, excited by local forces or a diffuse pressure field, has been computed using various techniques: - In the low frequency domain, the finite element method has been used to compute the structural behaviour, while the acoustic radiation has been computed by using the boundary element method and the finite element method with approximated boundary conditions to simulate the free field conditions. - In the high frequency domain, the computation has been made using the SEA method, with an approximation of the radiation coefficients. -In the medium frequency domain, both techniques have been used and compared. Software used where VA-One, from ESI, and Code-Aster, from EDF. The paper presents the numerical results obtained, associated to the computing time. Approximated methods can be largely faster than 'exact' ones (finite elements associated with boundary elements) and, for engineering use, represent a good compromise between quality and efficiency.

\section{$5: 20$}

1pSAa13. Structure-borne modeling of a vehicle in the mid-frequency range using Virtual SEA: experimental validation. Denis Thenail (PSA Peugeot Citroën, Route de Gisy, 78943 Vélizy-Villacoublay Cedex, France, denis.thenail@mpsa.com), Julien Baratier (Faurecia AST, Center of Acoustic Technology, Dämmstoffwerk 100, 38524 Sassenburg, Germany, julien.baratier@faurecia.com), Arnaud Duval (Faurecia AST, Center of Acoustic Technology, Dämmstoffwerk 100, 38524 Sassenburg, Germany, arnaud.duval@faurecia.com), Gérard Borello (InterAC, 10 impasse BordeBasse, ZA. La Violette, 31240 L'Union, France, gerard.borello@interac.fr), Laurent Gagliardini (PSA Peugeot Citroën, Route de Gisy, 78943 Vélizy-Villacoublay Cedex, France, laurent .gagliardini@mpsa.com)

Virtual SEA is a modeling process using FE computations to build an SEA model including equivalent masses, modal densities, and CLF, but excluding DLF since damping modeling in the mid-high frequency range is still an open issue. This technique, previously proposed by some of the authors, is applied to a production vehicle in the range $200-1000 \mathrm{~Hz}$. The actual vehicle is simultaneously measured at a subset of the FE nodes. The automated sub-structuring provided by Virtual SEA (20 subsystems at $630 \mathrm{~Hz}$ ) is used to favorably position 64 sensors on the body. Next, an experimental SEA procedure is performed: a full transfer matrix is measured between more than 1000 excitation (hammer) locations and the sensors. In order to compensate for structural heterogeneity, input mobilities are measured at every point and used to normalize the transfer matrix As all measurement points are associated to FE nodes, computed input mobilities can be compared to measurements. Finally, the SEA model identification is carried out for both experimental and virtual SEA. As far as damping (DLF) can only be known experimentally, comparisons of the numerical and experimental approach only concern the orher SEA parameters (CLFs, modal densities ...) and transfer functions.

\section{$5: 40$}

1pSAa14. Measurement of SEA damping loss factor for complex structures. Maxime Bolduc (Univ. de Sherbrooke, Mechanical Engineering Depart., 2500 Boulevard de 1'Université, Sherbrooke, QC J1K 2R1, Canada, maxime.bolduc@usherbrooke.ca), Noureddine Atalla (Univ. de Sherbrooke, Mechanical Engineering Depart., 2500 Boulevard de 1'Université, Sherbrooke, QC J1K 2R1, Canada, Nouredinne.Atalla @Usherbrooke.ca)

Statistical Energy Analysis has become extremely popular in the transportation industry. As a prediction tool, it offers appealing advantages such as its wide frequency range and short computational time, which conventional methods do not offer. Critical parameter in every SEA model, the 
damping characteristics of the subsystems must be determined by way of experimentations. A variety of different techniques of measuring the damping loss factor were developed. These techniques can be divided into three main groups: (i) method based on the identification of modal damping by curve-fitting frequency response function, (ii) decay techniques based ondetermination of the reverberation time and, (iii) steady-state techniques involving measurements of power input method much closely related to the definition of SEA since its starting point is the power balance. This work presents an experimental study of these techniques for various structures such as flat metallic panels, aircraft side walls (ribbed curved panels) and sandwich-composite panels in both low and high damping configurations (additions of damping materials, sound packages in both single wall and double wall configurations, mounting effects, ...). In particular, a critical discussion is made on the implementation, advantages and drawbacks of each method regarding its use in SEA modeling of complex structures.

\section{6:00}

1pSAa15. Transmission loss of a panel with tuned vibration absorbers. Carl Q. Howard (The University of Adelaide, School of Mechanical Engineering, North Terrace, 5005 Adelaide, Australia, carl.howard@ adelaide.edu .au)

The transmission loss of a rectangular panel with numerous discrete tuned vibration absorbers is investigated both theoretically and experimentally. The 49 tuned vibration absorbers have a linear distribution of resonance frequencies and were arranged in grid pattern on the rectangular plate. The transmission loss of the plate was measured between two reverberation chambers and was compared with theoretical predictions. In addition, theoretical predictions and experimental measurements were conducted on a similar system where each vibration absorber was replaced with the equivalent mass of the absorber. The numerical computations were conducted on a distributed computing network comprising 200 desktop computers. The results indicate the improvement in sound transmission loss that can be achieved, greater than merely increasing the thickness of the panel (mass law), and also provides a data set for comparison with predictions involving the use of fuzzy-structure theory.

\section{6:20}

1pSAa16. A 'concatenation' study of wave energy transport among three coupled plates. Nick Wolff (Dept. of Mechanical Science and Engineering, University of Illinois, 1206 W Green St. MC 244, Urbana, IL 61801, USA, nlwolff@uiuc.edu), Richard Weaver (Dept. of Physics, University of Illinois, Urbana, IL 61801, USA, r-weaver@uiuc.edu)

We address the problem of constructing predictions for the diffuse transport and distribution of mean spectral acoustic energy density in an undamped complex linear structure, without appeal to SEA and by taking information gleaned from short time cost-effective, direct numerical simulations (DNS). A numerical model is constructed consisting of three finite plates coupled by sets of springs. Coupling is chosen to be sufficiently strong to avoid localization and sufficiently weak that the notion of substructures remains valid. Numerical simulations of transient responses to each of several sources are carried out; responses are recorded at each of several receivers. The resulting time-domain diffuse waveforms are analyzed for the work done at each source, and the transient spectral energy density at each receiver at times shortly after the action of the sources. This information is used in a 'concatenation' ansatz to predict the evolution and distribution of spectral energy density at these receivers at later times. The resulting prediction is compared favorably with the actual energy densities observed in the DNS at late times. It is described how the results also apply directly to the more conventional but closely related problem of damped systems and/or steady state input. Work supported by NSF CMS- 0528096 .

\section{6:40}

1pSAa17. Modification of an interface parameter between sub-system and vehicle: case of a fan system attached to the front end of a car. Saul Mapagha (CEVAA, Technopole du Madrillet, 2 Rue Joseph Fourier, 76800 Saint Etienne du Rouvray, France, s.mapagha@cevaa.com), Vincent
Martin(Institut Jean Le Rond d'Alembert, UMR CNRS 7190, UPMC, 2 Place de la Gare de Ceinture, 78210 Saint-Cyr l’Ecole, France, vmartin@ccr .jussieu.fr)

The car industry, as well as many others, is constantly undergoing modifications to comply security and comfort regulations. Among the major causes for concern is the integration of sub-systems on vehicles. In fact, when a sub-system runs on a vehicle, it can lead to vibrations on the body of the car and thus acoustical radiations, causing trouble for the passengers, and also outside the vehicle. The frequency response functions (FRF)-based substructuring technique and impedances coupling methods are used to predict the forces entering the vehicle from those measured on a test bed. When a given constraint on the forces entering the vehicle is not satisfied, the car supplier may choose to modify the interface parameters between the substructures. In this paper an analytical approach to filter the entering forces by elastic suspension is proposed. An application is given by way of numerical experiments on a fan system attached to the front end of a car, both subsystems being identified through measurements.

\section{7:00}

1pSAa18. Sound radiation induced force vibration of submerged and fluid filled multilayered spherical shell. Sadeq Malakooti (Acoustics Research Laboratory, Dept. of Mechanical Engineering, Iran University of Science and Technology, Narmak, 16844 Tehran, Iran, s_malakooti@mecheng .iust.ac.ir), Seyyed M. Hasheminejad (Acoustics Research Laboratory, Dept. of Mechanical Engineering, Iran University of Science and Technology, Narmak, 16844 Tehran, Iran, hashemi@iust.ac.ir), Nader Mohammadi (Univ. of Tehran, School of Mechanical Engineering, 1439957131 Tehran, Iran, nmohamady@ut.ac.ir)

Nowadays sound radiation from spherical shells is attracted for aviation industry. In this paper, the general approach is presented for predicting a coupled fluid solid interaction problem. Therefore, a fully elasticity solution is applied in conjunction with laminated shell model for considering of the sound field over the shell. For each plies the elasticity coefficients are assumed to be constant. The main objectives of this work are investigating the influence of internal and external acoustic mediums and also radially inhomogeneity on acoustic performance of the spherical shell. Numerical results are presented for a three layered spherical shell. For each excitation frequency, sound pressure over zenith angle in polar coordinate is plotted. Also pressure levels against excitation frequency for resonance spectroscopy are investigated.

\section{$7: 20$}

1pSAa19. Particular analysis of vibration energy transmission in connections of thin plates. Jacek Cieslik (AGH-UST University of Science and Technology, Al. Mickiewicza 30, 30-059 Krakow, Poland, cieslik @agh.edu.pl)

Analyses of energy balance in the mechanical systems are done by the quantitative and qualitative assessment. Qualitative assessment is possibly by the use of energy flow descriptors and observation of energy density distribution in the mechanical structure. The work presents the results of investigation of welded connections of rectangular steel plates. The aim of the analysis was the quantitative estimation of vibrational energy transmitted though the welded connection depending on the inclination angle of plates. The structural intensity was used as the parameter for the analysis. Method of structural intensity evaluation enabled the elaboration of intensity vector field. Unification of shape and size of finite elements made possible the summation of only the magnitude of intensity. Summation was performed in the plate's cross sections far from the places of excitation and the damping. The detailed information on vibration energy flow was achieved. The obtained results of calculation give the quantitative information on amount of energy transmitted, reflected and the damped in welded joints of plates. The analyzed case was intentional to show the utility of intensity method in diagnostics of joints in mechanical constructions. The calculation results were verified experimentally with application of thermograph measurements of stress and vibration velocity. 


\title{
Session 1pSAb
}

\section{Structural Acoustics and Vibration and EURONOISE: General Topics in Structural Acoustics and Vibration I}

\author{
Wolfgang Kropp, Cochair \\ Chalmers University of Technology, Division of Applied Acoustics, Gothenburg, SE-41296, Sweden \\ Sean Wu, Cochair \\ Wayne State Univ., 5050 Anthony Wayne Dr., Detroit, MI 48202, USA
}

\section{Contributed Papers}

1:00

1pSAb1. Efficient computation of the radiated sound power of vibrating structures using a modal approach. Nicolas Roy (Top Modal, 5, rue de la ZA de Ribaute, 31130 Quint-Fonsegrives, France, nicolas.roy@topmodal.fr), Marc Lapi (DGA/DCE/CTSN, Site du Mourillon, BP 28, 83800 Toulon Armées, France, marc.lapi@dga.defense.gouv.fr)

The optimal design of structures in terms of noise control is of great interest in many fields such as automotive, aerospace, and naval. Since design strategies require simple design parameters, the sound power is often used to characterize the sound radiated from a structure. The CTSN in collaboration with TOP MODAL has recently developed a software tool to efficiently compute the radiated sound power of a vibrating structure using modes obtained by finite element analysis. The modal analysis has been enhanced to take into account the presence of fluid cavities and damping elements via the introduction of residual modes. The radiated sound power is computed from the volume velocities of the vibrating surface using a lumped parameter method requiring no explicit modeling of the acoustic medium. The modal contributions to the total sound power may also be calculated. The underlying methods are described including the improved coupled fluid-structure modal analysis and the computation of the radiated sound power. Next an overview of the tool's architecture is presented. Finally, several industrial applications are presented to illustrate the features and interest of the tool.

\section{$1: 20$}

1pSAb2. High resolution modal analysis. Kerem Ege (Laboratoire de Mécanique des Solides, Ecole Polytechnique, 91128 Palaiseau Cedex, France, kerem.ege@1ms.polytechnique.fr), Xavier Boutillon (Laboratoire de Mécanique des Solides, Ecole Polytechnique, 91128 Palaiseau Cedex, France, boutillon@1ms.polytechnique.fr), Bertrand David (Télécom Paristech (ENST) / TSI - CNRS LTCI, 46, rue Barrault, 75634 Paris Cedex 13, France, bertrand.david@enst.fr)

The Fourier-transform-based traditional modal analysis techniques perform poorly when modal overlap $\eta$ exceeds $30 \%$, due to the $\Delta \mathrm{T} . \Delta \mathrm{f}$ limitation. In view of filling the gap between the low- and the highfrequency domains where modal analysis and statistical methods respectively apply, a technique based on the high resolution analysis algorithm ESPRIT (Roy \& Kailath, IEEE Transactions on Acoustics Speech and Signal Processing, 37(7), 984-995, 1989) has been developed. A pulselike force is repeatedly applied to the structure and the response is measured in a number of points. In each point, the impulse response of the structure is retrieved by deconvolving the pulselike force and filtering the response with the result. In a second step, the number of modes in the reconstructed impulse response is evaluated by the novel procedure ESTER (Badeau et al., IEEE Transactions on Signal Processing, 54(2), 450-458, 2006) and various preconditioning techniques are applied. The ESPRIT algorithm finally extracts the modal frequencies, damping factors, and complex amplitudes at each point. Two applications are presented: the separation of twin modes of a square plate $(\eta$ $=200 \%$ ) and the partial modal analysis of a $1620 \times 1000 \times 5 \mathrm{~mm}$ aluminum plate up to a modal overlap $\eta=70 \%$.
$1: 40$

1pSAb3. Vibration of a submarine hull under harmonic propeller-shaft excitation. Mauro Caresta (University of New South Wales, 2052 Sydney, Australia, maurorestaca@yahoo.it), Nicole J. Kessissoglou (University of New South Wales, 2052 Sydney, Australia,n.kessissoglou@unsw.edu.au)

A model to describe the dynamic response of submarine hull to a harmonic propeller shaft excitation is presented. The submarine is modeled as a ring stiffened cylindrical shell, with bulkheads and end caps. The stiffeners are introduced using a smeared approach. The bulkheads are modeled as circular plates with in plane and bending motion, and the end closures are modeled as truncated conical shells. External fluid loading is introduced to take into account the interaction of the structure with the acoustic medium. The propeller introduces a harmonic varying force in both the axial and transverse directions. The force is transmitted to the structure through the shaft that is connected to the end plate of the cylindrical hull and supported by the conical end cap. The axial component excites the axisymmetric modes of the structure. The transverse force component excites the hull through the conical shell and excites the higher circumferential modes. Since these modes are mainly flexure in nature, they can result in a high noise signature level. Results are presented in terms of FRFs calculated analytically and compare the axisymmetric vibration caused by the axial excitation and the asymmetric response due to the transverse component of the force.

\section{2:00}

1pSAb4. Application of guided flexural waves in immersed plates to aquatic propulsion of mono-hull marine vessels. Victor $\mathrm{V}$. Krylov (Loughborough University, Department of Aeronautical and Automotive Engineering, Ashby Road, LE11 3TU Loughborough, UK, V.V.Krylov@lboro.ac.uk), Ewan Porteous (Loughborough University, Department of Aeronautical and Automotive Engineering, Ashby Road, LE11 3TU Loughborough, UK, wan2001@ hotmail.com)

The present paper describes the results of the experimental investigation of a small-scale mono-hull model boat propelled by a localised flexural wave propagating along the plate of finite width forming the boat's keel. Forward propulsion of the boat was achieved through flexural wave propagation in the opposite direction, which is similar to the aquatic propulsion used in nature by stingrays. The model boat under consideration underwent a series of tests both in a Perspex water tank and in the experimental pool. In particular, the forward velocity of the boat has been measured for different frequencies and amplitudes of the flexural wave. The highest velocity achieved was $32 \mathrm{~cm} / \mathrm{s}$. The thrust and propulsive efficiency have been measured as well. The obtained value of the propulsive efficiency in the optimum regime was $51 \%$. This indicates that efficiency of this type of aquatic propulsion is comparable to that of dolphins and sharks (around 75\%) and to that of a traditional propeller (around 70\%). In contrast to a propeller though, the wavelike aquatic propulsion has the following advantages: it does not generate underwater noise and it is safe for people and marine animals. 
1pSAb5. The structural acoustic properties of stiffened shells. Yu Luan (DTU, Building 352, Oersted. DTU, 2800 Lyngby, Denmark, yl @ oersted.dtu.dk)

Plates stiffened with ribs can be modeled as homogeneous isotropic or orthotropic plates, and modeling such an equivalent plate numerically with, say, the finite element method is, of course, far more economical in terms of computer resources than modelling the complete, stiffened plate. This is important when a number of stiffened plates are combined in a complicated structure composed of many plates. However, whereas the equivalent plate theory is well established there is no similar established theory for stiffened shells. This paper investigates the mechanical and structural acoustic properties of curved shells with stiffening ribs. Finite element simulations and experimental data will be compared and discussed.

\section{2:40}

1pSAb6. Multi-bay double panel system with heterogeneous blanket treatment: A comparison between theory and experiment. Kamal Idrisi (Virginia Tech, Mechanical Engineering, 143 Durham 0238, Blacksburg, VA 24061, USA, idrisi@vt.edu), Marty Johnson (Virginia Tech, Mechanical Engineering, 143 Durham 0238, Blacksburg, VA 24061, USA, martyj@vt.edu), Alessandro Toso (Virginia Tech, Mechanical Engineering, 143 Durham 0238, Blacksburg, VA 24061, USA, aletoso@vt.edu)

This study is part of an effort to improve the low frequency performance of acoustic blankets used to reduce the noise inside aircraft cabins. This is achieved by embedding small masses inside the poro-elastic layer such that they act like distributed mass spring damper systems. These mass-springdamper systems can then be designed to reduce the sound transmission through the double panel system at low frequencies where traditional poroelastic materials have little effect. A mathematical model of a multi-bay double panel system with frames, stringers, an acoustic cavity and porous/mass layer was developed using impedance and mobility methods (IMM). The multi-bay double panel system includes four skin pockets with four HG blankets of different dimensions such that the interaction between the panels can be analyzed. The predicted responses of the source and receiver panel due to a point force are validated with experimental measurements. The results indicate that proper tuning of the mass insertions can improve the broadband noise reduction below $500 \mathrm{~Hz}$ with less than $10 \%$ added mass without losing the performance of the acoustic foam at high frequencies.

\section{3:00}

1pSAb7. Acoustic radiation from vibrating panels subject to high-frequency broadband excitation. Donald B. Bliss (Duke University, Mechanical Engineering and Materials Science, 148B Hudson Hall, Durham, NC 27708, USA, dbb@duke.edu), Linda P. Franzoni (Duke University, Mechanical Engineering and Materials Science, 148B Hudson Hall, Durham, NC 27708, USA, franzoni@duke.edu)

Radiation from a baffled panel subject to high-frequency broadband excitation is studied. If the panel flexural waves are subsonic, the radiation comes from edges. For supersonic waves the entire surface is important. Characterization of the radiation is surprisingly straightforward in the highfrequency broadband limit, with simple analytical expressions for directivity patterns. For subsonic waves, a series expansion of the surface velocity wave number transform, convergent in the radiating region, can be reinterpreted physically as singularity functions along the panel perimeter, namely the delta function and its derivatives. This interpretation leads to monopole, dipole, and higher-order edge radiators with relative strengths that depend on surface-wave Mach number. Both propagating and evanescent structural waves contribute to the radiation. A proper physical explanation of the radiation is provided both in physical space and transform space. The common interpretation of edge radiation in terms of uncancelled volumetric sources is not correct; the higher order edge singularities are very significant. For low structural damping, this approach leads to a very simple way to calculate the radiated field. The relationship between radiation and structural power flow is potentially useful in energy-intensity based prediction methods with application to vehicle interior noise. (Sponsor NSF)
1pSAb8. Active noise control at a moving location in a modally dense three-dimensional sound field using virtual sensing. Danielle J. Moreau (The University of Adelaide, School of Mechanical Engineering, North Terrace, 5005 Adelaide, Australia, danielle.moreau@adelaide.edu.au), Ben S. Cazzolato (The University of Adelaide, School of Mechanical Engineering, North Terrace, 5005 Adelaide, Australia, benjamin.cazzolato@adelaide.edu.au), Anthony C. Zander (The University of Adelaide, School of Mechanical Engineering, North Terrace, 5005 Adelaide, Australia, anthony.zander@adelaide.edu.au)

Local active noise control systems generate a zone of quiet at the physical error sensor using secondary sources to cancel the acoustic pressure at the sensor location. The resulting zone of quiet is generally limited in size and as such, placement of the physical error sensor at the location of desired attenuation is required, which is often inconvenient. Virtual acoustic sensors overcome this by projecting the zone of quiet away from the physical error sensor to a remote location. While virtual acoustic sensors have shown potential to improve the performance of local active noise control systems, it is, however, likely that the desired location of maximum attenuation is not spatially fixed. The work described here presents a virtual sensing method capable of tracking a desired location in a modally dense three-dimensional sound field. The developed algorithm has been experimentally verified in a three-dimensional enclosure and the experimental results are presented.

\section{3:40}

1pSAb9. Active modal control in rooms - practical approach. Pierre-Jean Rene (Acoustical Engineering \& Research, Ch. de la Vuachère, 20D, 1012 Lausanne, Switzerland, pjrene@a-e-r.ch), Hervé Lissek (Ecole Polytechnique Fédérale de Lausanne, EPFL STI LEMA, Station 11, CH 1015 Lausanne, Switzerland, herve.lissek@epfl.ch)

Engineers dealing with noise reduction in habitations close to transportation traffic or industrial facilities encounter several problems to decrease noise level in rooms at low frequencies. Passive materials and current building construction knowledge enable to avoid noise transmission in habitations at medium and high frequencies and the regulations based on the $\mathrm{dBA}$ scale can often be respected. But these regulations do not take into account the real unpleasantness of noise for the inhabitants who are still disturbed by low frequency noise. Because of the modal behavior of rooms, air-borne and structure-borne noise generate high sound pressure level at the first modal frequencies, even with small amount of energy. The aim of this work is to decrease noise level in rooms at these specific and most annoying frequencies. Usual techniques of room acoustics and acoustic insulation reach their limits and cannot be improved due to cost and volume constraints. To reach our goal, active modal control seems to be the best way. The principle of the AMC is to decrease only the 2 or 3 first modal frequencies with a unique microphone-controller-loudspeaker system. Several results are presented for different excitations and room configurations. The controller setup has to be the more autonomous and flexible system as possible.

\section{4:00-4:20 Break}

\section{$4: 20$}

1pSAb10. Resonant frequencies of a cantilevered timoshenko beam with an attached general point-connected vibratory system. Robert $\mathrm{M}$. Koch (NUWC, 1176 Howell Street, Code 8232, Bldg. 1302, Newport, RI 02841-1708, USA, kochrm@npt.nuwc.navy.mil)

The literature is full of technical papers that have examined the free vibration of cantilevered Bernoulli-Euler beams with both rigidly and elastically-attached point masses at the free end. Additionally, much work has been published in the last ten years on cantilevered Timoshenko beams (which includes the effects of shear deformation and rotatory inertia) with a similar, yet more restricted, set of free end boundary conditions. Rossit and Laura published in the Journal of the Acoustical Society of America in 2001 a formulation for a Timoshenko beam with a simple single DOF spring-mass system attached to the free end. The present paper extends Rossit and Laura's mathematical formulation to account for a general, damped, multi- 
DOF system point-connected to a cantilevered Timoshenko beam's free end. To demonstrate the formulation, mathematical relations and numerical results for a single DOF damped system and both damped/undamped 2DOF vibration absorbers are presented.

\section{4:40}

1pSAb11. Damage detection in composite laminates using coin-tap method. Sung Joon Kim (Korea Aerospace Research Institute, 45 EoeunDong, Youseong-Gu, 305-333 Daejeon, Republic of Korea, yaelin@kari.re $. \mathrm{kr})$

The "coin-tap" test has the ability to indicate damage in a composite structural element due to a localized change of stiffness. The change in vibration signature may be detected by ear or more precisely by measurement of the dynamic contact force. It has been shown that the characteristics of radiated sound from a structure during a tap are changed by the presence of defect beneath the surface of the composite structure. For structurally radiated noise, the sound field is directly coupled to the structural motion. Therefore, impact response analysis should be computed. In this study, the radiated sound induced by impact is obtained by solving the Rayleigh integral equation. Experimental results for the delaminated composite structure are presented and correlations between analytical predictions and measured data are shown. It is shown that the sound based tap test is useful and practical diagnostic tool for detecting localized delamination in composite laminates

\section{5:00}

1pSAb12. Identification of noise sources by means of inverse finite element method using measured data. Matthias Weber (Helmut-Schmidt-Universität - Universität der Bundeswehr Hamburg, Holstenhofweg 85, 22043 Hamburg, Germany, mweber@hsuhh.de), Thomas Kletschkowski (Helmut-Schmidt-Universität - Universität der Bundeswehr Hamburg, Holstenhofweg 85, 22043 Hamburg, Germany, thomas.kletschkowski@hsuhh.de), Delf Sachau (Helmut-Schmidt-Universität - Universität der Bundeswehr Hamburg, Holstenhofweg 85, 22043 Hamburg, Germany, sachau@ @ hsuhh.de)

Identification of noise sources in airplane cabins proves to be difficult particularly at low frequencies. A new approach reconstructs the spatial distribution of sound pressure and particle velocity based on the inverse finite element (FE) method. This procedure requires measurements in the cavity of the cabin. If all sound sources are located on the boundary of the cabin, the equation system resulting from a matching FE model can be resorted in such way that computation of the unknown boundary data is possible, even with distorted measurement data. The method is explained using a simplified 2D laboratory experiment consisting of a flat sound-hard bounded rectangle with a loudspeaker included in the boundary. A corresponding FE model with given boundary conditions is verified by mapping the sound pressure in the test bed. The inner part of the measurement data is used to compute the boundary values. Regularization methods are applied to find an optimal solution. To prepare a verification of this technique in real world conditions, the sound field in the cavity of an airplane mock-up (Airbus A340) exited by both an interior and exterior noise source is mapped with a custom-built microphone array. Taking advantage of the results of the $2 \mathrm{D}$ examination, the current status of this verification process is shown.

\section{$5: 20$}

1pSAb13. Reconstruction of Rayleigh-Lamb dispersion spectrum based on noise obtained from an air-jet forcing. Eric Larose (LGIT CNRS - Université Joseph Fourier, Maison des Géosciences, 1381 rue de la Piscine, BP 53, $38041 \quad$ Grenoble, France, Eric.Larose@obs.ujf-grenoble.fr), Philippe Roux (LGIT - CNRS - Université Joseph Fourier, Maison des Géosciences, 1381 rue de la Piscine, BP 53, 38041 Grenoble, France, philippe.roux@obs.ujf-grenoble.fr), Michel Campillo (LGIT - CNRS - Université Joseph Fourier, Maison des Géosciences, 1381 rue de la Piscine, BP 53, 38041 Grenoble, France, Michel .Campillo@obs.ujf-grenoble.fr)

The time-domain cross-correlation of incoherent and random noise recorded by a series of passive sensors contains the impulse response of the medium between these sensors. By using noise generated by a can of com- pressed air sprayed on the surface of a plexiglass plate, we are able to reconstruct not only the time of flight but the whole waveforms between the sensors. From the reconstruction of the direct $A_{0}$ and $S_{0}$ waves, we derive the dispersion curves of the flexural waves, thus estimating the mechanical properties of the material without a conventional electromechanical source. The dense array of receivers employed here allow a precise frequency-wave number study of flexural waves, along with a thorough evaluation of the rate of convergence of the correlation with respect to the record length, the frequency, and the distance between the receivers. The reconstruction of the actual amplitude and attenuation of the impulse response is also addressed in this paper [Larose et al, J. Acoust. Soc. Am 122 (2007)].

\section{$5: 40$}

1pSAb14. Interaction of a pair of complex dynamical systems under impact conditions. Joseph F. Vignola (Catholic University, 620 Michigan Ave., Washington, DC 20064, USA, vignola@cua.edu), John A. Judge (Catholic University, 620 Michigan Ave., Washington, DC 20064, USA, judge@cua.edu), John McCoy (Catholic University, 620 Michigan Ave., Washington, DC 20064, USA, mccoy@ cua.edu)

An important class of complex dynamical systems has members that are comprised of a dominant simple system, made complex by attaching a large number of significantly less massive subsystems. The response of such systems to a simple forcing that acts on the dominant system has received considerable attention. A simple forcing is understood here to be represented by a time series that has very limited support when represented in either temporal or spectral space. Little attention has be paid to the response of such systems to a complex forcing, understood to be one represented by a time series that has extended support when represented in both temporal and spectral spaces. In the reported study, the interaction of two systems drawn from the described class, under impact conditions are investigated. Significantly, the action of each system on the other during the time interval of their contact is represented by an interaction force that is complex in the sense described. The investigation is accomplished via numerical simulation, physical experiments and analysis.

\section{6:00}

1pSAb15. Reduction of radiated sound from a continuum using low-damping vibration absorbers. Zenzo Yamaguchi (Kobe Steel, Ltd., 1-5-5, Takatsukadai, Nishi-ku, 651-2271 Kobe, Hyogo, Japan, zyamaguc@purdue.edu), Akio Sugimoto (Kobe Steel, Ltd., 1-5-5, Takatsukadai, Nishi-ku, 651-2271 Kobe, Hyogo, Japan, sugimoto.akio@kobelco.com), Yoshio Yano (Kobe Steel, Ltd., 1-5-5, Takatsukadai, Nishi-ku, 651-2271 Kobe, Hyogo, Japan, yano.yoshio @kobelco.com), J. Stuart Bolton (Ray W. Herrick Labs., School of Mech. Eng., Purdue University, 140 S. Martin Jischke Drive, West Lafayette, IN 47907-2031, USA, bolton@purdue.edu)

When reducing the sound radiated from a resonant structure, it is necessary to ensure that the driving frequency does not correspond to any of the system resonance frequencies. In addition, it may be desirable to use dynamic vibration absorbers to reduce the response at the driving frequency. In this paper, a low-damping vibration absorber is studied for solving noise radiation problems from a resonant, light-gauge structure driven by a force whose frequency and amplitude are constant and whose frequency does not match any of the system natural frequencies. When the driving frequency and the structure's natural frequency are different, the structure cannot be represented by as a SDOF system. Therefore, the conventional design method, based on equivalent mass, is inapplicable. In this paper, a design method based on the mobility of the structure is proposed. By means of the proposed method, the sound power radiated from a simply supported beam with an attached absorber is calculated and it leads to the following results: 1) When the absorber is installed at the driving point, it reduces the sound power as predicted; 2) An increase in radiated noise may occur when the absorber is not installed at the driving point. 


\section{6:20}

1pSAb16. A fluid level sensor using A0 Lamb wave mode. Fouad Boubenider (University of Science and Technology Houari Boumediene (USTHB), Laboratoire de Physique des Matériaux BP32, El Alia 16111, DZ Alger, Algeria, fboubenider@yahoo.fr)

The aim of this study was to examine the feasibility of creating a fluid level sensor operating in A0 Lamb wave mode. The experimental sensor is composed of a plate of stainless steel 1.3 meters high, in which, an A0 Lamb wave mode is generated. The choices of the material, the plate thickness and the wave vibration mode are all important. We therefore plotted the phase and group velocity curves, and the transversal and longitudinal displacements versus the product frequency $\mathrm{x}$ thickness. These curves enabled us to visualize the best target point for the excitation energy on a suitably guided wave mode at an appropriate frequency; this point is referred to as the operating point. When the chosen wave mode is generated, the echo from the reflection at the guide-water interface is easily detected by the transmitterreceiver transducer and can be worked efficiently.

MONDAY AFTERNOON, 30 JUNE 2008

ROOM 240, 1:00 TO 4:00 P.M.

\title{
Session 1pSCa
}

\section{Speech Communication: Speech Technology I}

\author{
Deliang Wang, Chair \\ Ohio State University, Department of Computer Science \& Engineering, 2015 Neil Ave., Columbus, OH 43210, USA
}

\section{Contributed Papers}

\begin{abstract}
1:00
1pSCa1. Automatic testing of speech understanding. Tom Francart (ExpORL, Dept. Neurosciences, K.U. Leuven, Herestraat 49 bus 721, O. \& N2, B-3000 Leuven, Belgium, tom.francart@med .kuleuven.be), Marc Moonen (ESAT/SISTA, K.U. Leuven, Kasteelpark Arenberg 10, B-3001 Leuven, Belgium, marc.moonen@esat .kuleuven.be), Jan Wouters (ExpORL, Dept. Neurosciences, K.U. Leuven, Herestraat 49 bus 721, O. \& N2, B-3000 Leuven, Belgium, jan.wouters @ med.kuleuven.be)
\end{abstract}

Speech reception tests are commonly administered by manually scoring the oral response of the test subject. This requires a test supervisor to be continuously present, which can be avoided by having the subject type the response on a computer keyboard and automatically scoring the response. However, spelling errors may then be counted as recognition errors, and hence influence the test results. We demonstrate an autocorrection approach based on two scoring algorithms to cope with spelling errors. The first algorithm deals with sentences and is based on word score. The second algorithm deals with single words and is based on phoneme score. Both algorithms are evaluated with a corpus of typed answers based on three different Dutch speech materials. The percentage of differences between the autocorrection algorithm and the manual correction was calculated, in addition to the mean difference in speech recognition threshold between automatic and manual scoring. The sentence correction algorithm performed at higher accuracy than commonly obtained with these speech materials. The word correction algorithm performed better than the human operator. Both algorithms can be used in practice and allow speech reception tests with open set speech materials over the internet.

\section{$1: 20$}

1pSCa2. A comparison between two methods for speech transmission quality assessment with noise simulation at receiver's side. Marcel Wältermann (Deutsche Telekom Laboratories, Berlin Institute of Technology, Ernst-Reuter-Platz 7, 10587 Berlin, Germany, marcel.waeltermann @ telekom.de), Nicolas Côté (France Télécom, 2 avenue Pierre Marzin, 22300 Lannion, France, nicolas.cote@ orange-ftgroup.com), Alexander Raake (Deutsche Telekom Laboratories, Berlin Institute of Technology, Ernst-Reuter-Platz 7, 10587 Berlin, Germany, alexander.raake @ telekom.de), Sebastian Möller (Deutsche Telekom Laboratories, Berlin Institute of Technology, Ernst-Reuter-Platz 7, 10587 Berlin, Germany, sebastian.moeller@telekom.de)

Two auditory experiments are presented that were designed in order to compare two different methods for the assessment of speech quality when noise at listener's side is present: (1) Headphone presentation with samples pre-recorded over a head and torso simulator, and (2) test participants located in a noisy environment, rendered by a 4.1 loudspeaker system. Realworld types of background noise were employed as well as a variety of user terminals. The correlation between corresponding overall quality scores amounts to $r=0.9$. Hence, a headphone presentation can replace a loudspeaker noise simulation in principle, leading to the consequence that a simpler experimental set-up is sufficient for assessing the speech quality when noise is present at the receiver's side. However, there exist certain differences between both quality scores. For example, in most cases clean wideband $(50-7000 \mathrm{~Hz})$ and standard narrow-band $(300-3400 \mathrm{~Hz})$ speech are rated slightly lower if the samples are presented over a headphone and noise is simultaneously present. Since the separation between speech and noise is potentially easier when the noise is played back over loudspeakers, participants may mainly judge the speech rather than the overall quality, leading to higher scores for the clean channel conditions.

\section{$1: 40$}

1pSCa3. Predicting speech quality under noise in wideband speech transmission. Juan-Pablo Ramirez (Deutsche Telekom Laboratories, Berlin Institute of Technology, Ernst-Reuter-Platz 7, 10587 Berlin, Germany, juan-pablo.ramirez@telekom.de), Alexander Raake (Deutsche Telekom Laboratories, Berlin Institute of Technology, Ernst-Reuter-Platz 7, 10587 Berlin, Germany, alexander.raake@telekom.de), Marcel Wältermann (Deutsche Telekom Laboratories, Berlin Institute of Technology, Ernst-Reuter-Platz 7, 10587 Berlin, Germany, marcel.waeltermann@telekom.de), Sebastian Möller (Deutsche Telekom Laboratories, Berlin Institute of Technology, Ernst-Reuter-Platz 7, 10587 Berlin, Germany, sebastian.moeller@telekom.de)

Prediction of users' opinion of speech quality in telecommunication is of a major issue in speech transmission planning. For this purpose, reliable models, such as the E-model (ITU-T Rec. G.107, 2005) were developed for narrow-band (NB) transmission. Meanwhile, telephony using the Internet Protocol (VoIP) has enabled an enlargement of the transmission band, introducing wideband (WB; $50-7000 \mathrm{~Hz}$ and beyond) to every day communication. The quality improvement thanks to the bandwidth extension was found to be $29 \%$, as compared with NB [3]. Consequently, an updated planning model is required to assess quality in WB speech transmission. We performed two listening only tests to study the quality impairment due to signal attenuation, different noise types and level and various codecs. The tests showed that user's quality perception in case of WB was similar to NB for the noisy conditions. However, considerably higher judgments were given to WB than to NB for the low-noised conditions. With increasing noise level, the quality of NB and WB become more and 
more alike, and quality differences between codecs decrease. Following the lines of thoughts of the E-model, we propose a model assessing the quality impairment in WB speech transmission resulting from noise and/or signal attenuation.

\section{2:00}

1pSCa4. Effect of degradations' distribution in a corpus test on auditory ratings. Nicolas Côté (France Télécom, 2 avenue Pierre Marzin, 22300 Lannion, France, nicolas.cote@orange-ftgroup.com), Virginie Durin (France Télécom, 2 avenue Pierre Marzin, 22300 Lannion, France, virginie.durin@orange-ftgroup.com)

Speech quality of telecommunications systems is usually evaluated thanks to auditory tests, which must be carried out in accordance with ITU-T Recommendations. In these tests, subjects are asked to assess the quality of speech sample by giving a score on a five-level scale. The averaging of subjects' scores yields the Mean Opinion Score (MOS) which represents the speech quality for a given condition. However, MOS values can be strongly influenced by many factors. In this paper, we focus on a specific bias: the distribution of the impairments in the stimuli set. In effect the rating of a narrow-band stimulus $(300-3400 \mathrm{~Hz})$ may have a lower quality score in a mixed-band set (mixed of narrow-band and wideband (50-7000 $\mathrm{Hz}$ ) conditions) than in a purely narrow band set. This effect is detailed through results of auditory tests recently carried out. Consequently, the validity of MOS value is theoretically limited within a test which prevents MOS comparison between different tests. Finally, two solutions are proposed to avoid this effect: first, an improvement of auditory tests methodology and then, a new approach to assess speech quality, based on the subject's behaviour.

\section{2:20}

1pSCa5. Influence of informational content of background noise on speech quality evaluation for VoIP application. Adrien Leman (France Telecom, 2, Avenue Pierre Marzin, 22300 Lannion, France, adrien.leman@orange-ftgroup.com), Julien Faure (France Telecom, 2, Avenue Pierre Marzin, 22300 Lannion, France, julien.faure@orange-ftgroup.com), Etienne Parizet (Laboratoire Vibrations Acoustique, Insa Lyon, 25 bis, av. J. Capelle, 69621 Villeurbanne Cedex, France, etienne.parizet@insa-lyon.fr)

With the rise of mobile communication systems, the background noise in the speaker's environment and its interaction with VoIP network impairment affects speech quality perception. This effect should be taken into account in nonintrusive models in order to improve accuracy of end user perception measurement. The aim of this study is to determine the impact of information contained in background noise (background speech, environmental sources) on speech quality perception. A subjective test on the speech quality perception in real network conditions has been done comparing the effect of stationary background noise mainly due to transmission equipment (electrical humming or blowing noises) with the effect of nonstationary environmental noise (public place, traffic noise, background conversation). Interactions between these different background noise condition and realistic network impairments (coders and packets loss) were also studied. The speech quality was evaluated through the Mean Opinion Score determined from an Absolute Category Rating method.

\section{2:40}

1pSCa6. Speech perception in noise with binary gains. Deliang Wang (Ohio State University, Department of Computer Science and Engineering, 2015 Neil Ave., Columbus, OH 43210, USA, dwang@cse.ohio-state.edu), Ulrik Kjems (Oticon A/S, Kongebakken 9, DK-2765 Smørum, Denmark, uk@Oticon.dk), Michael S. Pedersen (Oticon A/S, Kongebakken 9, DK-2765 Smørum, Denmark, msp@Oticon.dk), Jesper B. Boldt (Oticon A/S, Kongebakken 9, DK-2765 Smørum, Denmark, jeb@Oticon.dk), Thomas Lunner (Oticon Research Centre Eriksholm, Kongevejen 243, DK-3070 Snekkersten, Denmark, TLU @Oticon.dk)

For a given mixture of speech and noise, an ideal binary time-frequency mask is constructed by whether SNR within individual time-frequency units exceeds a local SNR criterion (LC). With linear filters, co-reducing mixture
SNR and LC does not alter the ideal binary mask. Taking this manipulation to the limit by setting both mixture SNR and LC to minus infinity produces an output that contains only noise with no target speech at all. This particular output corresponds to turning on or off the filtered noise according to a pattern prescribed by the ideal binary mask. Our study was designed to test on speech intelligibility of noise gated by the ideal binary mask obtained this way. It is observed that listeners achieve nearly perfect speech recognition from gated noise. Only sixteen filter channels and a frame rate of one hundred Hertz are sufficient for high intelligibility. The results show that, despite a dramatic reduction of speech information, a pattern of binary gains provides an adequate basis for speech perception in noise.

\section{3:00}

1pSCa7. Low-dimensional, auditory feature vectors that improve vocal-tract-length normalization in automatic speech recognition. Jessica J. Monaghan (Centre for the Neural Basis of Hearing, Department of Physiology, Development and Neuroscience, University of Cambridge, Downing Site, CB23EG Cambridge, UK, jjmm2@cam.ac.uk), Christian Feldbauer (Centre for the Neural Basis of Hearing, Department of Physiology, Development and Neuroscience, University of Cambridge, Downing Site, CB23EG Cambridge, UK, feldbauer@tugraz.at), Tom C. Walters (Centre for the Neural Basis of Hearing, Department of Physiology, Development and Neuroscience, University of Cambridge, Downing Site, CB23EG Cambridge, UK, tcw24@cam.ac.uk), Roy D. Patterson (Centre for the Neural Basis of Hearing, Department of Physiology, Development and Neuroscience, University of Cambridge, Downing Site, CB23EG Cambridge, UK, rdp1@cam.ac.uk)

Human speech recognition is robust to large changes in vocal tract length (VTL) but automatic speech recognition is not. In an effort to improve VTL normalization, an auditory model was used to derive formantlike features from syllables. The robustness supported by these auditory features was compared to the robustness provided by traditional MFCCs (MelFrequency Cepstral Coefficients), using a standard HMM recognizer (Hidden-Markov-Model). The speech database consisted of 180 syllables, each scaled with the vocoder STRAIGHT to have a wide range VTLs and glottal pulse rates. Training took place with syllables from a small, central range of scale values. When tested on the full range of scaled syllables, average performance for MFCC-based recognition was $73.5 \%$, with performance falling close to $0 \%$ for syllables with extreme VTL values. The feature vectors constructed with the auditory model led to much better performance; the average for the full range of scaled syllables was $91 \%$, and performance never fell below $65 \%$ even for extreme combinations of VTL and GPR. Moreover the auditory feature vectors contain just 12 features whereas the standard MFCC vectors contain 39 features. Research supported by the UK-MRC (G0500221) and EOARD (FA8655-05-1-3043).

\section{$3: 20$}

1pSCa8. A Portable robot audition software system for multiple simultaneous speech signals. Hiroshi G. Okuno (Kyoto University, Graduate School of Informatics, Yoshida-Honmachi, Sakyo, 606-8501 Kyoto, Japan, okuno@i.kyoto-u.ac.jp), Shunichi Yamamoto (Kyoto University, Graduate School of Informatics, Yoshida-Honmachi, Sakyo, 606$8501 \quad$ Kyoto, Japan, shunichi@kuis.kyoto-u.ac.jp), Kazuhiro Nakadai (Honda Research Institute Japan Co., Ltd., 8-1 Honcho, Wako, 351-0114 Saitama, Japan, nakadai@jp.honda-ri.com), Jean-Marc Valin (CSIRO ICT Center, Cnr Vimiera \& Pembroke Rds, NSW 2122 Marsfield, Australia, jean-marc.valin@csiro.au), Tetsuya Ogata (Kyoto University, Graduate School of Informatics, Yoshida-Honmachi, Sakyo, 606-8501 Kyoto, Japan, ogata@kuis.kyoto-u.ac.jp), Kazunori Komatani (Kyoto University, Graduate School of Informatics, YoshidaHonmachi, Sakyo, 606-8501 Kyoto, Japan, komatani@kuis.kyoto-u.ac.jp)

Since a robot is deployed in various kinds of environments, the robot audition system should work with minimum prior information on environments to localize, separate and recognize utterances by multiple simultaneous talkers. For example, it should not assume either the number of speakers, the location of speakers for sound source separation (SSS), or specially 
tuned acoustic model for automatic speech recognition (ASR). We developed a portable robot audition that uses eight microphones installed on the surface of robot's body such as Honda ASIMO, and SIG-2 and Robovie-R2 at Kyoto University with different microphone configurations. The system integrates SSS and ASR by using the Missing-Feature Theory. For SSS, we use Geometric Source Separation (GSS) [Parra 2002] and multichannel post-filter [Cohen 2002] to separate each utterance. Since separated speech signals are distorted due to interfering talkers and sound source separation, multichannel post-filter enhanced speech signals. At this process, we create a missing feature mask that specifies which acoustic features are reliable in time-frequency domain. Multiband Julius [Nishimura 2004], a missingfeature-theory based ASR, uses this mask to avoid the influence of unreliable features in recognizing such distorted speech signals. The system demonstrated a waitress that accepts meal orders placed by three actual human talkers and a voice "Rock-Scissors-Paper" game referee that decides who wins the game.

\section{3:40}

1pSCa9. Ambiguity in the recognition of phonetic vowels when using a bone conduction microphone. Véronique Zimpfer (ISL, 5 rue du Général Cassagnou BP 70034, 68301 Saint Louis, France, veronique
.zimpfer@isl.eu),Karl Buck(French German Institut of Saint Louis (ISL), 5 rue du Général Cassagnou, 68301 Saint-Louis, France, karl.buck@isl.eu)

When speaking, not only air conducted noise is generated, but also vibrations can be recorded at different places on the head using accelerometers. The bone conduction microphones are less sensitive to noise than regular acoustical microphones, they are usable in harsh environments and they are compatible with head equipment such as NBC protection devices. This paper reports the first results of a study designed to evaluate the differences in perception between speech recorded via a acoustic microphone and speech recorded using bone conduction. These differences may cause of a bad intelligibility of bone conduction communication, even if presented in an undisturbed environment. We study the recognition of ten French phonetic vowels recorded by a bone conduction microphone. A listening test is designed to show the confusions of phonetic vowels when listening to speech picked up by an air or bone conduction microphone. The tests show confusion between the vowels [i] [y] [u]. All of these have the frequency of their first formant in common. The sonograms are distinctly different when recorded with an aero-acoustic microphone, become almost identical if the bone conducted speech is analyzed. Moreover, the confusions of the phonetic vowels depend on the speaker.

\title{
Session 1pSCb
}

\section{Speech Communication: Measurement of Sociophonetic Variation in Speech}

\author{
Ewa Jacewicz, Cochair \\ The Ohio State University, 110 Pressey Hall, 1070 Carmack Road, Columbus, OH 43210, USA \\ Jonathan Harrington, Cochair \\ Institute of Phonetics and Speech Processing, University of Munich, Munich, 80799, Germany
}

\section{Invited Papers}

1:00

1pSCb1. Inter- and intra-speaker variation: some methodological consequences for sociophonetic research. Gerard J. Docherty (Newcastle University, School of ECLS, KG VI Building, NE3 1YB Newcastle upon Tyne, UK, g.j.docherty@ncl.ac.uk), Paul Foulkes (University of York, Department of Language \& Linguistic Science, Heslington, YO10 5DD York, UK, pf11@ york.ac.uk)

In recent years there has been a rapidly growing awareness of the importance of sociophonetic variation for advancing models of speech production, perception and learning. Meanwhile within sociolinguistic research there has been an equally rapidly evolving recognition that full accounts of the social marking functions of speech communication require greater focus on the manner in which individuals deploy phonetic resources variably as a reflection of the fluid identities which they project across the diverse interactions which they participate in. The aim of this talk is to explore some of the methodological consequences of these developments. We explore the ways in which an approach to sociophonetic variation which rightly focuses on how it plays out in the performance of individual speakers can be reconciled with the development of models of speech production and perception which to date have typically been driven by what is found to be common across individual speakers/listeners and much less by those factors which differentiate them.

$1: 20$

1pSCb2. Sociophonetic methods for studying substratal effects and new dialect formation. Erik R. Thomas (North Carolina State University, Department of English, Box 8105, Raleigh, NC 27695-8105, USA, ethomas@ social.chass.ncsu.edu)

Studies of substratal effects in the formation of new language varieties have seldom combined both acoustic sophistication and the examination of a broad suite of pronunciation features. This study provides an example of how such work can be conducted. The study focuses on Pearsall, Texas, a community in southern Texas in which Mexican Americans have formed an established community for four generations and exhibit a shift from Spanish dominance to English dominance. A variety of segmental and prosodic variables are examined, both for different generations of Mexican Americans and for Anglo speakers of the contact dialect, and the variables are compared statistically. Results show that some Spanish interference features are discarded, while others take on a life of their own as markers of Mexican American identity. 
1pSCb3. Modeling dialect-related variability in American English plosives. Thomas Purnell (University of Wisconsin, 1168 Van Hise, 1220 Linden Drive, Madison, WI 53706, USA, tcpurnell@wisc.edu)

Socially motivated variation in speech often lies in the differential use of phenomena described in identical or near-identical terms. This paper investigates the hypothesis that dialect differences may result from the relative distribution of an acoustic characteristic with respect to trading relations with other co-varying characteristics, or by variations in the characteristic within regions of linguistic stability. Plosive variation among European American and African American English speakers in the upper Midwest is examined. Speakers from both dialect groups may produce plosives instead of interdental fricatives in syllable initial position, and devoice or glottalize apical plosives in syllable final position. Additionally, speakers of both dialects have been observed to hypervoice voiced plosives word-initially. The current experiment explores the boundary characteristics of a trading relations model within the plosive system of both dialect groups. The specific question addressed by the paper is whether the range of plosive variation suggests a shared property of nonstandard dialects, or whether the relation among acoustic measures is dialect specific. Results are discussed in light of acoustic, perceptual and categorical stability.

\section{2:00}

1pSCb4. The acoustic and perceptual bases of diachronic /u:/-fronting in Standard Southern British. Jonathan Harrington (Institute of Phonetics and Speech Processing, University of Munich, 80799 Munich, Germany, jmh@phonetik.uni-muenchen.de), Felicitas Kleber (Institute of Phonetics and Speech Processing, University of Munich, 80799 Munich, Germany, kleber@phonetik.uni-muenchen.de), Ulrich Reubold (Institute of Phonetics and Speech Processing, University of Munich, 80799 Munich, Germany, reubold@phonetik.uni-muenchen.de)

In a recent experiment concerned with diachronic/u:/-fronting in Standard Southern British, it was shown both that the /u:/ category boundary was perceptually retracted for older compared with younger listeners of this variety, and that they compensated perceptually to a greater extent for the coarticulatory fronting effects of the preceding consonant on /u:/ in words like used. In the present study, these perceptual differences were related to the same speakers' productions of words containing a /u:/ nucleus in a fronting (e.g., 'used') and non-fronting (e.g., 'swoop') contexts. The relative acoustic distance of /u:/ between front and back vowels was measured in a threedimensional space formed by applying the discrete-cosine-transformation to the entire F2-trajectory shape separately for each speaker. Compatibly with their perceptual responses, older listeners had a more retracted /u:/ on this measure and exhibited a greater coarticulatory influence of the preceding consonant. The mechanism of this sound change is considered both from the point of view of a shift in the perceptual compensation for coarticulation and recent developments in episodic models of speech perception

\section{2:20}

1pSCb5. Speech processing and dialect variation in the American Midwest. Cynthia G. Clopper (Ohio State University, 1712 Neil Ave, Oxley Hall 222, Columbus, OH 43210, USA, clopper.1@osu.edu)

The Midwestern United States is divided into two dialect regions: North and Midland. The Northern dialect is characterized by the clockwise rotation of the low and low-mid vowels in the acoustic-phonetic vowel space. The Midland dialect is characterized by back vowel fronting and the merger of the low back vowels. A series of experiments exploring the effects of dialect variation on spoken word recognition has revealed systematic linguistic processing costs associated with these dialect-based vowel system differences for Midwestern listeners. While word recognition performance in noise is equally accurate for both dialects, the patterns of errors that listeners produce reveal systematic confusions between phonologically different vowels that overlap in the acoustic-phonetic space across the two dialects. In addition, speeded lexical classification performance is highly accurate for both dialects, but performance is slower for Northern vowels than Midland vowels of the same phonological class. The results from these studies suggest that even in the absence of striking intelligibility differences between the two dialects, processing costs associated with the perception of dialect variation can be observed using traditional speech perception and spoken word recognition tasks.

$$
\text { 2:40 }
$$

1pSCb6. Analysis of total vowel space areas in three regional dialects of American English. Robert A. Fox (The Ohio State University, 110 Pressey Hall, 1070 Carmack Road, Columbus, OH 43210, USA, fox.2@osu.edu), Ewa Jacewicz (The Ohio State University, 110 Pressey Hall, 1070 Carmack Road, Columbus, OH 43210, USA, jacewicz.1@osu.edu)

The acoustic characteristics of vowel systems in different varieties of American English are greatly affected by regional variation. Given the significant positional differences of vowels within the acoustic space across regional dialects of English, one should expect that the size and extent of the vowel space is also affected by this type of variation. Traditionally, the size of the acoustic vowel space has been measured as the triangular area defined by the three corner vowels. An obvious weakness of this approach is that it underestimates the actual "working space" of vowel system in that the onsets and/or offsets of other vowels are often found outside this triangular area. This paper proposes a procedure to estimate the area of a complete vowel space, taking into account dynamic formant pattern of all vowels and diphthongs. Complete vowel space areas are calculated for individual speakers and compared across three distinct regional varieties (representing Inland North, Southern Appalachian, and a Midland variety). The comparison also examines possible changes to vowel space area as a function of speaker generation (younger and older adults).

\section{3:00}

1pSCb7. Regional variation in vowels and vowel systems: normalization and optimization. Roeland Van Hout (Department of Linguistics - Radboud University, Post Box 9103, 6500 HD Nijmegen, Netherlands, hout@let.ru.nl)

We made an acoustical description of regional variation patterns in the vowel system of Dutch spoken in the Netherlands and Flanders. The speech material consisted of read monosyllabic utterances in a neutral consonantal context, representing the vowels of 
Dutch. A discriminant analysis applied on the raw measurements to classify each speaker into one of the eight regions involved (sociogeographic variation) gave a correct regional classification for $72.0 \%$ of the 160 speakers. When normalization procedures were applied, the percentages of proper classification increase to $82.5 \%$. Several questions have to be answered though. Which normalization procedure is the best one and why, and how can we be sure that specific parts of sociophonetic variation are not distorted by the normalization procedure? We will present additional materials collected on the same set of speakers in reading aloud a strictly controlled list of words containing all Dutch vowels in different consonantal contexts. Another question is how to reduce the amount of information used in a normalization procedure. The $\mathrm{z}$ transformation (Lobanov) performs well, but is rather expensive in the amount of information needed. Can we minimize the information needed and yet uncover the patterns of sociophonetic variation?

1pSCb8. A new speaker-intrinsic vowel formant frequency normalization algorithm for sociophonetics. Anne Fabricius (Roskilde University, Department of Culture and Identity, Postboks 260, DK4000 Roskilde, Denmark, fabri@ruc.dk), Dominic Watt (University of York, Department of Language \& Linguistic Science, Heslington, YO10 5DD York, UK, dw539@york.ac.uk)

This paper evaluates the strengths and weaknesses of a speaker-intrinsic vowel formant frequency normalization algorithm initially proposed in Watt and Fabricius (2002) and modeled by Thomas and Kendall (2007) for direct comparison with other normalization algorithms. We evaluate the merits of the new routine as a sociophonetic research tool relative to those of two well-known speakerintrinsic methods documented in Lobanov (1971) and Nearey (1977) through comparisons of the values of two parameters: degree of overlap of vowel spaces and vowel space area ratios. Measurements of angles and Euclidean distances between pairs of points in the vowel space (a method presented in Fabricius 2007) also provide a comparative parameter revealing how different algorithms model the vowel space. The study uses two existing datasets: 1) a corpus of RP vowels compiled from Hawkins and Midgley (2005) and Moreiras (2006) and 2) previously unpublished data from Aberdeen, northeast Scotland.

\section{$3: 40$}

1pSCb9. Stability and variation in the pronunciation of French: A corpus-based approach. Jacques Durand (Laboratoire Cognition, Langues, Langage, Ergonomie (CLLE), Université Toulouse - Le Mirail \& CNRS, 5, Allées Antonio Machado, 31058 Toulouse, France, jacques.durand@univ-tlse2.fr), Noel Nguyen (LPL, Aix-Marseille Université \& CNRS, 29, avenue Robert Schuman, 13100 Aix-en-Provence, France, Noel.Nguyen@lpl-aix.fr)

This contribution relates to the project "La Phonologie du Français Contemporain" (PFC), the largest and most ambitious survey of modern French ever conceived. The PFC project involves over thirty researchers from a variety of countries and aims at the recording, partial transcription and phonetic analysis of over 600 speakers from the francophone world. It aims at a broad coverage of varieties of contemporary French by selecting groups of speakers from approximately 60 different locations in the francophone world. So far around 600 speakers have already been recorded in various parts of the francophone world beside France, including Belgium, Burkina Fasso, Canada, Côte d'Ivoire, Louisianne, and Switzerland. The recordings involve formal and informal conversation, the reading aloud of a word-list and a passage. Current work conducted on the database ranges from auditory exploitation of the data to automatic speech recognition via classical experimental phonetics. We address theoretical and empirical issues related to liaison as well as vowel systems, and examine sociogeographical variation and changes in progress in these respects within French, using a variety of quantitative methods for the empirical assessment of phonological hypotheses. 


\title{
Session 1pSCc
}

\section{Speech Communication: General Topics in Speech Communication I (Poster Session)}

\author{
Erika Levy, Cochair \\ Biobehavioral Sciences, Teachers College, Columbia University, New York, NY 10027, USA \\ Susanne Fuchs, Cochair \\ ZAS, Schuetzenstr. 18, 10117 Berlin, Germany \\ Patti Adank, Cochair \\ F.C. Donders Centre for Cognitive Neuroimaging, University College London, London WCIN 3AR, UK
}

\begin{abstract}
All posters will be on display from 4:20 p.m. to 6:00 p.m. To allow contributors an opportunity to see other posters, contributors of odd-numbered papers will be at their posters from 4:20 p.m. to 5:10 p.m. and contributors of even-numbered papers will be at their posters from 5:10 p.m. to 6:00 p.m.
\end{abstract}

\section{Contributed Papers}

1pSCc1. Acoustic validation of the analysis of diadochokinesis in normal speech using the motor speech profile program. Yu-Tsai Wang (National Yang-Ming University, School of Dentistry, Rm. 411, No. 155, Sec. 2, Li-Nong St. Beitou District, 11221 Taipei, Taiwan, yutsaiwang@ym.edu.tw), Yuh-Mei Chung (Dept. of Physical Medicine and Rehabilitation, Taipei Veterans General Hospital, No. 201, Sec. 2, Shih-Pai Rd., 11217 Taipei, Taiwan, ymchung@vghtpe.gov.tw), Ray D. Kent (Waisman Center, Rm. 435, University of Wisconsin-Madison, 1500 Highland Avenue, Madison, WI 53705-2280, USA, kentray@waisman.wisc.edu), Hsiu-Jung Lu (National Yang-Ming University, School of Dentistry, Rm. 411, No. 155, Sec. 2, Li-Nong St. Beitou District, 11221 Taipei, Taiwan, hsiujung_lu@ hotmail.com)

The purpose of this study is to evaluate the suitability, reliability, and concurrent validity of the analysis of diadochochinesis (DDK) in normal speech using a computer program, Diadochokinetic Rate Analysis (DRA) in the KayPENTAX Motor Speech Profile. Fifteen healthy participants were recorded at UW-Madison as they repeated various syllables as quickly and steadily as possible. The DDK samples were executed by the DRA protocol to generate immediate quantitative information at different thresholds and were also hand-measured. When the lowest peak intensity during consonantvowel syllables is lower than the highest peak intensity during intersyllable pauses, the DRA output is incorrect and the DDK sample is defined as nonexecutable. Analyses were based on the percentage of nonexecutable DDK samples and the comparisons of the results between repeated analyses at different thresholds and between automatic and manual measuring methods. Results: (1) One-ninth of the DDK samples was nonexecutable; (2) when the protocol was executable, the reliability at different thresholds and validity between different measuring methods were both satisfactory; and (3) the temporal variation between different measuring methods were larger than the intensity variation. Implications of the findings will be discussed in terms of the application of the DRA to general clinical purposes.

1pSCc2. Analysing cockpit and laboratory recordings to determine fatigue levels in pilots' voices. Claude Legros (Université de Toulouse-Le Mirail, Laboratoire d'Acoustique LAUTM, 5, allées Antonio Machado, 31058 Toulouse Cedex 1, France, legros@univ-tlse2.fr), Robert Ruiz (Université Toulouse II, LARA, 5 allée Antonio Machado, 31058 Toulouse Cedex, France, robert.ruiz@univ-tlse2.fr), Philippe Plantin De
Hugues(Ministère des Transports, Bureau Enquêtes Analyses, 200 rue de Paris, Bât 153, Aéroport du Bourget, 93352 Le Bourget Cedex, France, plantindehugues@bea-fr.org)

Analysis of pilots' voices was undertaken under controlled laboratory conditions, using aeronautical terminology, standard professional equipment, and a standardized recording environment. The aim of the experiment was to determine the way the acoustic characteristics of the voice are modified after a phase of sleep. The results, which are presented in this paper, show very significant variations in the dispersion parameters, like jitters, associated with the fundamental frequency. In an aircraft cockpit, the electroacoustic and environmental conditions are not so good. Therefore, it is essential to improve and adapt voice analysis methods to achieve reliable results. Cockpit Voice Recorder recordings have poor acoustic characteristics due to microphone quality and the signal to noise ratio, in addition to the non-predetermined vocabulary range. This paper will outline the modifications made to the laboratory methodology and the comparative results obtained, to improve the analysis of CVR recordings. Comparisons of the test recordings meant that it was feasible to determine, via voice analysis, the state of drowsiness of a pilot and, made possible the study of CVR recordings from accidents using new analytical techniques.

1pSCc3. The temporal location of rms peak in coarticulated vowels. Ewa Jacewicz (The Ohio State University, 110 Pressey Hall, 1070 Carmack Road, Columbus, OH 43210, USA, jacewicz.1@osu.edu), Robert A. Fox (The Ohio State University, 110 Pressey Hall, 1070 Carmack Road, Columbus, OH 43210, USA, fox.2@osu.edu)

The acoustic energy peak of the vowel, here defined as the rms peak, occurs typically before the temporal vowel midpoint. This study examines whether and how the location of the rms peak varies as a function of consonantal context and vowel duration. Four measures of rms peak location were explored: its distance (in ms) from vowel onset and from CVC-word onset (absolute measures) and its relative position (ranging from 0 to 100\%) within the vowel and within the CVC-word (relative measures). Analysis of eight American English vowels produced in ten consonantal contexts by twenty speakers shows that the two relative measures yielded the most comparable and consistent patterns. The relative location of rms peak varied significantly as a function of consonantal context. It occurred earlier (i.e., closer to vowel or word onset) in the context of fricatives (and not stops), voiced consonants (as opposed to voiceless), and labials (as opposed to 
alveolars). The temporal location of rms peak was also closer to the vowel onset (or word onset) for long vowels as opposed to short. Explanation of these patterns will be provided.

1pSCc4. Analysis of short-time speech transmission index algorithms Karen Payton (ECE Dept., UMass Dartmouth, 285 Old Westport Rd., Dartmouth, MA 02747, USA, kpayton@umassd.edu), Mona Shrestha (ECE Dept., UMass Dartmouth, 285 Old Westport Rd., Dartmouth, MA 02747, USA,g_mshrestha@umassd.edu)

Various methods have been shown to compute the Speech Transmission Index (STI) using speech as a probe stimulus (Goldsworthy \& Greenberg, J. Acoust. Soc. Am., 116, 3679-3689, 2004). Frequency-domain methods, while accurate at predicting the long-term STI, cannot predict short-term changes due to fluctuating backgrounds. Time-domain methods also work well on long speech segments and have the added potential to be used for short-time analysis. This study investigates the accuracy of two time-domain STI methods: envelope regression (ER) and normalized correlation (NC), as functions of window length, in various acoustically degraded environments with multiple talkers and speaking styles. Short-time STIs are compared with a short-time Theoretical STI, derived from octave-band signal-to-noise ratios and reverberation times. For windows as short as $0.3 \mathrm{~s}$, the ER and NC Methods track the short-time Theoretical STI and both the Theoretical and ER Methods converge to the long-term result for windows greater than $4 \mathrm{~s}$. Short-time STIs are also compared to intelligibility measurements on clear/conversational speech. Correlations between STI and intelligibility scores are high at the sentence and word levels and, consistent with the scores, short-time methods predict a higher average value of STI for clear than for conversational speech.

1pSCc5. An acoustic investigation into coarticulation and speech motor control: high vs. low frequency syllables. Frank Herrmann (University of Sheffield, Human Communication Sciences, 31 Claremont Crescent, S10 2TA Sheffield, UK, F.Herrmann@Sheffield.ac.uk), Sandra P. Whiteside (University of Sheffield, Human Communication Sciences, 31 Claremont Crescent, S10 2TA Sheffield, UK, s.whiteside@sheffield.ac.uk), Stuart Cunningham (University of Sheffield, Human Communication Sciences, 31 Claremont Crescent, S10 2TA Sheffield, UK, s.cunningham@sheffield.ac.uk)

Levelt \& Wheeldon's concept of the mental syllabary (1994) suggests that articulatory routines for syllables are stored in the form of gestural scores in a library. Cholin et al. (2006) found a syllable frequency effect on naming latency of nonsense words and interpret this as supporting evidence for the existence of such a syllabary. This paper presents a data set from a project investigating speech motor learning and coarticulation. Acousticbased coarticulation measures, such as F2 locus equations, are used to indirectly determine the degree of gestural overlap in articulatory movements. Greater degrees of coarticulation can be indicators of cohesive articulatory gestures and therefore, motor speech skill. It is hypothesised that high frequency syllables, as well trained articulatory gestures, should exhibit a greater degree of coarticulation, less variation, and shorter duration times than low frequency syllables. Fifteen native speakers of English listened to, and repeated 16 mono-syllabic stimuli which belonged to either of two categories: high and low frequency (CELEX). Acoustic measurements included the transitions of the first three formants (C-to-V coarticulation), transition times and syllable duration. Patterns of coarticulation were quantified using F2 locus equations and the absolute changes in formant frequency. The results are presented and discussed.

1pSCc6. Segmental and supra-segmental contributions to cross-language speech intelligibility. Melanie Preece-Pinet (University College London, Department of Phonetics and Linguistics, 4, Stephenson Way, NW1 2HE London, UK, m.pinet@ucl.ac.uk), Paul Iverson (University College London, Department of Phonetics and Linguistics, 4, Stephenson Way, NW12HE London, UK, p.iverson@ucl.ac.uk)

Non-native accents affect speech recognition in noise, and previous work has shown that intelligibility is modulated by listener-talker interaction; a matched linguistic background between the two seems to maximise intelligibility. The present study examined the contribution of segmental and supra-segmental cues, as well as how the degree of L2 experience affects non-native speech perception. A group of monolingual native English listeners and two groups of French native listeners with different amounts of L2 experience were presented with English sentences produced by native and non-native (French) speakers in noise. The stimuli were digitally processed to swap the pitch and segment durations between recordings of the same sentences produced by different speakers (e.g., imposing a French-accented prosody onto recordings made from English speakers). The results revealed that English listeners were more accurate at recognizing speech with English segments but were little affected by prosody; low proficiency French listeners were more accurate when the speech had both French-accented segments and prosody; and high proficiency French listeners varied in the cues that they used depending on the noise level. The use of segmental and supra-segmental cues thus appears to vary with language experience and listening context.

1pSCc7. A multidimensional acoustic analysis of vowels in two Polish dialects. Wieslawa Cholewa (UMR 7018, CNRS/Univ. Paris III, 19, rue des Bernardins, 75005 Paris, France, Wieslawa.Cholewa@univ-paris3.fr), Charalampos Karypidis (UMR 7018, CNRS/Univ. Paris III, 19, rue des Bernardins, 75005 Paris, France, ch _karypidis@yahoo.com)

In this paper, we attempt to examine the acoustic (oral) vowel spaces of two main Polish dialects: the Polish spoken in Cracow and that spoken in the wider region of Warsaw. A semi-spontaneous corpus consisting of four speakers (two male, two female) of each dialect was recorded with approximately 2000 vowel occurrences for each speaker. The segmentation was performed manually and was subsequently corrected with an intensity-based algorithm. The tokens were submitted to acoustic analyses, and two multidimensional representations were then prepared, one based on formants (accompanied with bandwidths and amplitudes) and the other on critical bands. Both representations included measurements for duration, F0, spectral center of gravity, skewness, and kurtosis. These values served as input for discriminant and principal component analysis which allowed us: (a) to compare the relative weight of each cue, (b) to isolate those parameters that allow a differentiation of the members of a vowel system (in a language such as Polish, F1 and F2 are traditionally the sole cues regarded as relevant), and (c) to detect any (dis)similarities between the two dialects in the three aforementioned aspects.

1pSCc8. Automatic data enhancement for language identification using voice generation. Aaron Lawson (RADC, Inc., 525 Brooks Road, Rome, NY 13441, USA, aaron.lawson.ctr@rl.af.mil), Matthew Linderman (Air Force Research Laboratory, 525 Brooks Road, Rome, NY 13441, USA, matthew.linderman@gmail.com), Michael Carlin (Air Force Research Laboratory, 525 Brooks Road, Rome, NY 13441, USA, Michael.Carlin@rl.af.mil), Allen Stauffer (RADC, Inc., 525 Brooks Road, Rome, NY 13441, USA, stauffar@clarkson.edu)

Approaches to LID require very large sets of training (often greater than six hours per language) for accurate results. This study looks at ways of automatically reducing the amount of training data required to train a LID model, while maintaining or increasing accuracy. Initial experiments found that speaker density, i.e., the number of speakers per time unit, had a very dramatic influence on the accuracy of models (absolute increase of 15\%). In order to accomplish the goal of increasing the number of speakers available for LID training without having to collect additional audio, the STRAIGHT algorithm was used to synthesize "novel" speakers for use in training language models for a LID system. The mean pitch and vocal tract length of the speaker in each LID training file was scaled to generate four additional voices per original speaker to artifically augment the training data. The resulting models yielded an improvement of $10 \%$ over the baseline system (from $80 \%$ to $90 \%$ ). This study shows that automatically generated speakers 
have a great impact on the accuracy of LID models, in this case an initial training set of 15 minutes per language was augmented and performed comparably to a set trained with six hours.

1pSCc9. Channel mitigation approach for automatic language identification. Allen Stauffer (RADC, Inc., 525 Brooks Road, Rome, NY 13441, USA, stauffar@clarkson.edu), Aaron Lawson (RADC, Inc., 525 Brooks Road, Rome, NY 13441, USA, aaron.lawson.ctr@rl.af.mil)

A major obstacle to overcome in language identification (LID) performance is the impact of varying channel conditions. In house experiments show that LID performance drops between $10-12 \%$ across channels. The focus of this project is to mitigate the impact of channel conditions and artifacts on LID, and to provide an understanding of how channel robust models may be created by combining data from across corpora. Our approach involved creating composite cross-channel language models from multiple corpora that were tested with data from three corpora whose results were compared to results obtained from same-channel and pure cross-channel experiments. Our hypotheses were that 1) same-channel models would be the most accurate, 2) purely cross-channel models would be considerably less accurate, and 3) composite model accuracy would fall in between that of the same-channel and cross-channel models. Results were surprising: while pure cross-channel tests performed the worst, with an average of $11 \%$ loss in accuracy, composite models outperformed matched condition models in tests by $0.5 \%$ to $15 \%$ for all four combinations tested. These findings provide evidence that cross-channel augmentation does not just make for more robust LID models across channel conditions, but improves model robustness within channel conditions as well.

1pSCc10. Newborn pain cry analysis based on pitch frequency tracking. Delphine Bard (Lund University, Division of Engineering Acoustics, John Ericsson väg 1, 22100 Lund, Sweden, delphine.bard@acoustics.lth.se), Per Runefors (Department of Paediatrics, Växjö Hospital, $351 \quad 85 \quad$ Växjö, Sweden, per.runefors@vaxjo.mail.telia.com), Einar Arnbjörnsson (Department of Paediatric Surgery, University Hospital Lund, 22100 Lund, Sweden, einar .arnbjornsson@telia.com)

The aim of the newborn pain cry analysis is to test the hypothesis that cry can be used as a tool to detect signs of nocioceptive pain. Previous studies applying signal processing techniques to analyze the sound of these cries have been done. The subject of this paper is to adapt and improve the original method with the help of new signal processing methods. The pitch frequency is extracted from the waveform of the recorded babies' cries using time domain methods. The fluctuations of this parameter are analyzed in terms of jitter. In particular, a sliding buffer approach is presented, as well as an improvement of the Average Mean Difference Function (AMDF). Comparison between original and news results has been done.

1pSCc11. Age effects of pitch-shifted auditory feedback on reflexive and volitional voice F0 control. Kyoko Nagao (Center for Pediatric Auditory and Speech Sciences, Nemours Biomedical Reserach, A.I. du Pont Hospital for Children, 1600 Rockland Road, Wilmington, DE 19803, USA, nagao@asel.udel.edu), Katie E. McCurdy (Indiana University, Dept. of Speech and Hearing Sciences, $200 \mathrm{~S}$ Jordan Avenue, Bloomington, IN 47405, USA, kemccurd@indiana.edu), Theresa A. Burnett (Indiana University, Dept. of Speech and Hearing Sciences, 200 S Jordan Avenue, Bloomington, IN 47405, USA, burnett@indiana.edu)

Research on voice fundamental frequency (F0) control suggests that when making a voluntary vocal response to a pitch change in voice auditory feedback, the pitch-shift reflex typically occurs before the volitional response is initiated. The current study examined the effects of aging on reflexive and volitional voice F0 responses to pitch-shifted auditory feedback. Thirty participants (age range 19 to 78 years) repeatedly sustained an /u/ vocalization at a steady pitch and loudness while listening to their voice fed backed over headphones. Once per vocalization, feedback pitch was randomly shifted upward or downward for 100 milliseconds and 20 or 100 cents. In the Sustain condition, participants attempted to ignore the pitch change in auditory feedback voice. In the Follow condition, participants immediately changed their pitch in the same direction as the auditory pitch shift they heard. Preliminary results from thirteen participants indicate that there appears to be no effect of aging on the first F0 responses for both magnitude and latency. Voluntary voice F0 changes were delayed in older participants compared to younger participants as expected due to age-related general slowing. Results broaden our understanding of typical reflexive and volitional voice control capabilities across the age span.

1pSCc12. Do Japanese speakers perceive nonexistent vowels in non-native consonant clusters? Seiya Funatsu (The Prefectural Univ. of Hiroshima, 1-1-71 Ujinahigashi Minami-ku, 734-8558 Hiroshima, Japan, funatsu@pu-hiroshima.ac.jp), Satoshi Imaizumi (The Prefectural Univ. of Hiroshima, 1-1 Gakuen-machi, 723-0053 Mihara, Japan, imaizumi@pu-hiroshima.ac.jp), Masako Fujimoto (The National Inst. for Japanese Language, 10-2 Midori-machi, 190-8561 Tachikawa, Japan, mfuji@ viola.ocn.ne.jp), Akira Hashizume (Hiroshima Univ., 1-2-3 Kasumi Minami-ku, 734-8553 Hiroshima, Japan, wj8uc6@bma.biglobe.ne.jp), Kaoru Kurisu (Hiroshima Univ., 1-2-3 Kasumi Minami-ku, 734-8553 Hiroshima, Japan, kuka422@hiroshima-u.ac.jp)

Vowel epenthesis is a well known phenomenon that non-native speakers insert epenthetic vowels inside non-native consonant clusters. Vowel epenthesis is assumed as perceptual "illusory vowels" (Dupoux et al. 1999). We analyzed vowel epenthesis shown by native Japanese speakers during reading and repetition tasks for non-native consonant clusters, and analyzed their brain responses using magnetoencephalographic methods. Under the reading task, in which subjects read English words and nonsense words, native Japanese speakers (eight females) inserted vowel /o/ after /t/ and /d/ in consonant clusters, and vowel /u/ after other consonants. Under the repetition task, in which subjects repeated utterances of a native English speaker, native Japanese speakers did not produce epenthetic vowels with few exceptions. The length of exceptional epenthetic vowels found under the repetition task was shorter than those under the reading task. The magnetoencephalographic mismatch responses were elicited by epenthetic vowels, suggesting that the native Japanese detected epenthetic vowels as real segments. Based on these results, we conclude that vowel epenthesis by native Japanese speakers did not arise from "illusory vowels", but rather from the difficulty of articulating consonant clusters which are not found in Japanese and/or Japanese phonological rules.

1pSCc13. Fine-phonetic variation in a computational model of word recognition. Odette Scharenborg (Centre for Language and Speech Technology, Radboud University Nijmegen, Erasmusplein 1, 6525 HT Nijmegen, Netherlands, O.Scharenborg@let.ru.nl)

There is now considerable evidence from psycholinguistic and phonetic research that fine-phonetic variation in the speech signal modulates human speech processing (HSP), and helps the listener segment a speech signal into syllables and words [e.g., Salverda et al., Cognition 90, 51-89 (2003)]. This kind of information also appears to help the human perceptual system distinguish short words (like 'ham') from the longer words in which they are embedded (like 'hamster'). Salverda et al. showed that the lexical interpretation of an embedded sequence is related to its duration; a longer sequence tends to be interpreted as a monosyllabic word more often than a shorter one. Until recently, no computational models of HSP existed that are able to model this fine-phonetic variation [Hawkins, J. of Phonetics 31, 373-405 (2003)]. In this paper, we present Fine-Tracker, a novel computational model of human word recognition that it is able to capture and use this finegrained acoustic-phonetic variation during speech recognition. Simulations using the recordings from the listener experiments by Salverda et al. show that Fine-Tracker is able to capture and use duration variation in the speech signal to distinguish short words from the longer words in which they are embedded. [Research supported by NWO.]

1pSCc14. Lombard speech: effects of task and noise type. Youyi Lu (Sheffield University, Computer Science Department, Regent Court, 211 Portobello St., S1 4DP Sheffield, UK, y.lu@dcs.shef.ac.uk), Martin 
Cooke(Sheffield University, Computer Science Department, Regent Court, 211 Portobello St., S1 4DP Sheffield, UK, m.cooke@dcs.shef.ac.uk)

An analysis of the effect of noise on speech production requires material recorded while undertaking realistic tasks in the presence of realistic noise backgrounds. This study examined speech produced in a number of types of "noise" in tasks with and without a communication factor. In one task, individual speakers were asked to speak aloud while solving "sudoku" puzzles, while in another task pairs of speakers solved these puzzles cooperatively. In both cases the background was quiet or contained one of three types of noise: competing talker, babble-modulated noise and speech-shaped noise. Individual digit words "one" to "nine" and speech/nonspeech sections were manually segmented for acoustic analysis. Both tasks produced Lombard effects observed in previous studies: increases in duration, energy, F0 and spectral centre of gravity. Relative to the non-communicative task, the presence of a communication factor led to words with shorter duration, more overall energy and higher spectral centre of gravity. Speakers actively avoided overlapping with the competing speech masker, with a stronger effect in the communicative task. These results suggest that speakers can, to some extent, compensate for both energetic and informational masking at the ears of the interlocutor.

1pSCc15. Differences in the time-course of accent adaptation: a comparison of adaptation to foreign-accented and unfamiliar regionally-accented speech. Bronwen G. Evans (Department of Phonetics and Linguistics, University College London, 4 Stephenson Way, NW12HE London, UK, bronwen.evans@ucl.ac.uk), Patti Adank (F.C. Donders Centre for Cognitive Neuroimaging, Kapittelweg 29, 6525EN Nijmegen, Netherlands, patti.adank@fcdonders.ru.nl)

Recent work in speech perception has demonstrated that listeners are able to rapidly adapt to foreign- accented speech (Clarke and Garrett, 2004) However, it is not clear whether adaptation to an unfamiliar regional accent operates in the same way. Although some studies have shown that listeners are able to adapt to different accents within the same language after only a short amount of exposure (e.g., Maye et al., in press), others have shown that listeners do not always alter their perceptual representations when listening to a non-native regional accent even if they are highly familiar with that accent (Evans and Iverson, 2004, 2007). In this study, we further explored perceptual adaptation to different accents by comparing the time course of adaptation to an unfamiliar regional accent with adaptation to foreign- accented speech. Listeners identified sentences in noise produced in either an unfamiliar regional accent (Glaswegian) or foreign-accented speech (Spanish-accented English). Preliminary results suggest a different pattern of adaptation for each accent: although listeners show greater adaptation to foreign- accented speech, they perform more poorly with foreignaccented speech overall and the rate of adaptation is slower.

1pSCc16. Production of Parisian French front rounded vowels by second-language learners. Erika S. Levy (Teachers College, Columbia University, Biobehavioral Sciences, Box 180, 525 W120th St., New York, NY 10027, USA, elevy@tc.columbia.edu), Franzo Law (CUNY Graduate Center, 365 5th Avenue, Program in Speech-Language-Hearing Sciences, New York, NY 10016, USA, flaw@gc.cuny.edu)

This study examined the effects of language experience and consonantal context on the production of Parisian French (PF) vowels by American English (AE) learners of French. A repetition task was performed, involving French vowels /y-œ-i-a-u/ uttered by a native speaker of Parisian French $(\mathrm{PF})$ in bilabial $/ \mathrm{bVp} /$ and alveolar $/ \mathrm{dVt} /$ contexts embedded in the nonsense word/phrase $/ \mathrm{raCVCa} /$. Three groups of native $\mathrm{AE}$ speakers participated: speakers without French experience (NoExp), speakers with formal French experience (ModExp), and speakers with formal and extensive immersion experience (HiExp). Production accuracy was assessed by native PF listeners' judgments and by acoustic analysis. Native PF listeners identified second-language (L2) learners' productions more accurately as a function of speakers' increased language experience, although /u/, /y/ and /œ/, even produced by the HiExp group, were frequently misidentified. A consonantal context effect was revealed, including / $\mathrm{u} /$ being misidentified more often in alveolar context than in bilabial context by all three groups. Overall, all groups distinguished front rounded $/ \mathrm{y} / \mathrm{from} / \mathrm{u} /$ in production, but often in a non-native manner, e.g., producing /y/ as /ju/. Individual differences, interactions between consonantal context and vowels, as well as implications for the perception-production link in L2 learning, are discussed.

1pSCc17. Development of assisting system for learning Chinese aspirated sounds. Akemi Hoshino (Toyama College of Maritime Technology, Ebie, Neria, 933-0293 Imizu, Toyama Prefecture, Japan, hoshino@toyama-cmt.ac.jp), Jianwu Dang (Japan Advanced Institute of Science and Technology, 1-1 Asahidai, Nomi, 923-1292 Ishikawa, Japan, jdang@jaist.ac.jp), Akio Yasuda (Tokyo University of Marine Science and Technology, 2-1-6 Etchujima, 135-8533 Tokyo, Japan, yasuda@kaiyodai.ac .jp)

Chinese aspirates are generally difficult for Japanese to pronounce and perceive. In order to assist Japanese students to learn Chinese, the authors attempt to build a system to automatically evaluate the pronunciation of Chinese aspirates by Japanese students. To do so, this study analyzed 21 singlevowel syllables of six different Chinese aspirates, bilabial, alveolar, velar, palatal, retroflex, and dental using two evaluation parameters: the voice onset time (VOT) and mean breathing power during VOT. The speech materials were uttered by nine Japanese students and nine native Chinese speakers. It is found that for a give aspirate, the longer the VOT is, the better the score is, as is generally said. But we found that an aspirate with a larger breathing power acquires good mark even with a shorter VOT in a certain range of VOT. The testing results showed that a combination of these two parameters can distinguish aspirated and unaspirated sounds quite well. To achieve an assisting system, we developed a method to automatically detect the VOT. To detect the burst of an aspirate, a filter bank was used to measure signal energy in frequency domain, and the burst timing was determined.

1pSCc18. Articulatory perturbation due to ultrasound imaging: an acoustic analysis. Rachael-Anne Knight (City University, Department of Language and Communication Science, Northampton Square, EC1V OHB London, UK, knight@city.ac.uk), Christina Villafana Dalcher (City University, Department of Language and Communication Science, Northampton Square, EC1V 0HB London, UK, cvdalcher@mac.com), Mark J. Jones (University of Cambridge, Phonetics Lab, Department of Linguistics, Raised Faculty Building, Sidgwick Avenue, CB3 9DA Cambridge, UK, markjjones@hotmail.com)

A safe, non-invasive imaging technique which captures articulatory movements in real time is the Holy Grail of articulatory analysis. Ultrasound imaging of the tongue seems suitable, albeit for a single articulator. However, ultrasound requires positioning a probe under the chin which may inhibit jaw movement. It is well known that speakers compensate rapidly for similar articulatory perturbations such as bite-blocks. Consequently, the images obtained by ultrasound may show compensatory articulations due to jaw inhibition, rather than natural articulations. This study assesses the effects of articulatory compensation during ultrasound imaging using acoustic analysis. The experiment compares acoustic data from three speech conditions: probe-free, probe held manually, probe fixed non-manually. It is hypothesised that sounds requiring a low jaw position, such as low vowels, velars, and velarised laterals, will be worst affected. High jaw sounds such as $/ \mathrm{s} /$ and $/ \mathrm{i} /$ are also assessed. Any differences between conditions are assumed to be due to compensation for jaw immobility rather than learned segment-specific register effects and are therefore unlikely to diminish as the subjects relax. Variability may diminish, however, as compensatory strategies become more practised. We expect to find some effects on certain segments, but that manual probe holding allows for more natural speech.

1pSCc19. Cortical mechanisms of speech perception in noise in elderly listeners. Edward R. Lee (Northwestern University, 860 Hinman Ave., Apartment 715, Evanston, IL 60202, USA, EdwardLee86@gmail .com), Patrick C. Wong (Northwestern University, 2240 Campus Drive, Evanston, IL 60208, USA, pwong@ northwestern.edu), Sumitrajit Dhar (Northwestern University, 2240 Campus Drive, Evanston, IL 60208, USA, s-dhar@northwestern.edu), Geshri M. Gunesekera (Northwestern 
University, 2240 Campus Drive, Evanston, IL 60208, USA, geshri-g@northwestern.edu), Rebekah A. Abel (Northwestern University, 2240 Campus Drive, Evanston, IL 60208, USA, r-abel@northwestern.edu)

The present study examines the cerebral hemodynamics (measured by fMRI) associated with listening to speech in noise in elderly listeners with relatively normal peripheral hearing. We asked subjects to identify single words in quiet and in two multi-talker babble noise conditions $(+20$ and -5 $\mathrm{dB}$ SNRs). The behavioral results indicated that subjects performed similarly between the Quiet and +20 SNR conditions, both in accuracy and reaction time, but were less accurate and responded slower in the -5 SNR condition. There were performance-independent and SNR-dependent effects in the brain. A contrast of the +20 SNR and Quiet conditions revealed bilateral (especially left) auditory and prefrontal activation in noise despite equal behavioral performance (performance-independent but noisedependent activations). As noise increased and behavioral performance decreased (as in the -5 SNR condition), activation in these regions increased. These results reveal a cortical network involving acoustic analysis, working memory, and auditory attention associated with speech perception in noise by the elderly. [Work supported by NIH.]

1pSCc20. Computer simulations of intraoral pressure patterns during voiced stop consonant production. Jorge C. Lucero (Univ. Brasilia, Dept. Mathematics, 70910-900 Brasilia - DF, Brazil, lucero@unb.br), Laura L. Koenig (Haskins Labs. and Long Island Univ., Brooklyn, New York, NY 11201-8423, USA, koenig@ haskins.yale.edu)

Several past studies have used laryngeal and/or upper vocal tract models to explore aerodynamic control during production of obstruent consonants, yet many aspects of such control remain unclear. Of particular interest in this work is how varying laryngeal and vocal tract dimensions such as occur among men, women, and children influence phonatory behavior and the time course of intraoral pressure (Pio) increase during voiced stop consonant production. To maintain phonation during a voiced stop closure, Pio must remain below the subglottal pressure. Physiological and modeling studies indicate that adult speakers actively increase supraglottal volumes during voiced stops, which slows the rate of Pio increase. Speakers with smaller vocal tracts may either be less successful in slowing the rate of Pio buildup, or else may perform more extreme volumetric changes to achieve similar end results. The current study uses data recently collected on voicing and Pio during stop production in men, women, and children. We use a modified two-mass model of the vocal folds coupled to a model of the upper vocal tract to reproduce the observed Pio patterns, and explore how the behavior of the system reflects differences in anatomical and physiological dimensions. [Work supported by MCT/CNPq-Brazil.]

1pSCc21. The production of Mandarin and American English vowels by Mandarin-speakers. Jia-Shiou Liao (Department of Applied Foreign Languages, Chung Shan Medical University, No.100, Sec. 2, Dah-Ching St., 402 Taichung, Taiwan, jsliao@csmu.edu.tw)

This study examines whether there is any correspondence between how Mandarin-speakers pronounce Mandarin vowels and how they pronounce those vowels' English equivalents and non-equivalents. Twenty Mandarin graduate students, who spoke English as a second language and had stayed continuously in America for at least two years, produced a series of American English vowels in $/ \mathrm{hVd} /$ contexts from a randomly ordered list. To approximate English syllable structure, they also read a list of $/ \mathrm{hVdV} /$ syllables containing Mandarin vowels in the first syllable. The vowel in the second syllable is always a schwa. First- (F1) and second-formant (F2) values of each Mandarin and English vowel were analyzed. The statistical results suggest that the distances between the Mandarin corner vowels in the vowel space are similar to those between the English ones. The acoustic characteristics of the English vowels with Mandarin equivalents do not differ significantly from those equivalents. For the other English vowels, the formant values most often differ from those of their closest Mandarin equivalents in only one formant or in neither. For instance, Mandarin sub- jects do not distinguish English /u/ from the near-close near-back vowel in either formant - attributable to the absence of the near-close near-back vowel in Mandarin.

1pSCc22. The influence of the feature (sonorant) in lexical access in Spanish. Danny R. Moates (Ohio University, Department of Psychology, Porter Hall, Athens, OH 45701, USA, moates@ohio.edu), Emilia A. Marks (Ohio University, Department of Modern Languages, Athens, OH 45701, USA, markse@ohio.edu), Dario Barrera (Universidad de Sevilla, Facultad de Filologia, Departmento de Filologia Inglesa, Palos de la Frontera, s/n, 41004 Sevilla, Spain, dario@us.es)

Does the feature (sonorant) influence access to lexical entries? In current feature geometry, the feature (sonorant) is a root node feature, distinguishing resonants from obstruents. Marks, et al. (2002) demonstrated its influence in lexical access using the word reconstruction task in Spanish. Participants heard a nonword, e.g., dirmar, and were asked to change it into a real word (firmar) by changing just one consonant. Errors in recovering the correct word (firmar) were much higher when the word contained an obstruent that had been replaced with a resonant (nirmar) than when the word contained an obstruent that had been replaced by another obstruent (dirmar). Obstruent target words were easier to recover when the target segment and the replacing segment matched on the feature [sonorant]. Why do obstruents show this matching effect? The present study subdivided obstruents into stops and fricatives. Two experiments compared them separately to resonants in the word reconstruction task. Are fricatives alone or stops alone responsible for the observed obstruent effect? Results showed greater errors in the mismatch condition for both fricatives and stops, but significantly greater for fricatives than for stops, suggesting that the mismatch effect observed for obstruents is largely based on fricatives.

1pSCc23. Syllable production rate in conversational speech. Lawrence J. Raphael (Adelphi University, 1 South Avenue, Garden City, NY 11530, USA, raphael@adelphi.edu)

Most acoustic analyses of speech are based on samples obtained from carefully constructed corpora that are read or recited by subjects in a laboratory setting. Recently, speech scientists have attempted to obtain speech samples that are representative of a more casual, conversational style than might be obtained in the laboratory. One available and plentiful source of such speech samples can be found in "talkradio" shows in which listeners engage in phone conversations with radio hosts. Phone-in programs receive calls from thousands of callers each day across the continental United States. Moreover, the listeners who call generally speak in an essentially natural, unself-conscious manner, exhibiting a broad range of rates and emotional states. This paper reports the results of a study of syllable production rate from samples of speech obtained from a variety of types of phone-in programs, including those focusing on sports, politics and the arts. The data reveal that syllable production rates (in syllables per second) range from 3.05 to 7.50 . Subjective judgements of speech rate by naive subjects were found to correlate only moderately with actual rates of syllable production.

1pSCc24. A temporally stable representation of power spectra of periodic signals and its application to $\mathrm{F} 0$ and periodicity estimation. Hideki Kawahara (Faculty of Systems Engineering, Wakayama University, 930 Sakaedani, 640-8510 Wakayama, Japan, kawahara@sys.wakayama-u.ac.jp), Masanori Morise (Faculty of Systems Engineering, Wakayama University, 930 Sakaedani, 640-8510 Wakayama, Japan, s055068@sys.wakayama-u.ac.jp), Toru Takahashi (Faculty of Systems Engineering, Wakayama University, 930 Sakaedani, 640-8510 Wakayama, Japan, tall@sys.wakayama-u.ac.jp), Ryuichi Nisimura (Faculty of Systems Engineering, Wakayama University, 930 Sakaedani, 640-8510 Wakayama, Japan, nisimura@sys.wakayama-u.ac.jp), Hideki Banno (Meijyo University, 501-1 Shiogamaguchi, Tenpakuku, 468-8502 Nagoya, Japan, banno@ccmfs.meijo-u.ac.jp), Toshio Irino (Faculty of Systems Engineering, Wakayama University, 930 Sakaedani, 640-8510 Wakayama, Japan, irino@sys.wakayama-u.ac.jp)

A unified framework for extracting F0 and periodicity is proposed based on a new formulation of STRAIGHT. Averaging power spectra, they are calculated using two time windows a half pitch period apart, yields a tempo- 
rally stable power spectrum for periodic signals (TANDEM spectrum). Applying consistent sampling theory also yields computationally efficient implementaiton of spectral envelope estimation used in STRAIGHT (STRAIGHT spectrum). Normalized TANDEM spectrum by STRAIGHT spectrum only represents periodicity information and can be used to detect periodicity both in the base-band (for F0 extraction) and other frequency bands (for mixed mode excitation). This unified framework provides a basis to represent the complex modes of voice excitation and enables greatly realistic remaking of extreme vocal expressions. [Supported by Grant in aids for scientific research and CrestMuse project.]

1pSCc25. Construction of MRI-based three-dimensional atlas of the human tongue for tongue modeling. Chao-Min Wu (Dept. Electrical Engineering, National Central University, \#300, Chung-Da Rd., 320 Chung-Li, Taiwan, wucm@ee.ncu.edu.tw), Sung-Yi Wang (Dept. Electrical Engineering, National Central University, \#300, Chung-Da Rd., 320 Chung-Li, Taiwan,945201073@cc.ncu.edu.tw)

The main purpose of this study is to build a MRI-based 3D tongue atlas for research on tongue morphometrics and physiological modeling of normal and disordered speech production. In this study, MRI data of 8 subjects (4 males and 4 females) were chosen from an orally-based MRI database of 20 male and 20 female college students without speech disorders. Sixteen landmarks were defined and selected from the 3D reconstructed MRI tongue images based on the subjects. Thin-plate spline analysis (TPS) was used to build a 3D tongue atlas for male and female subjects, respectively. Sagittal sections of the original MRI data were used to evaluate the accuracy of image registration and reconstruction. The resulted $3 \mathrm{D}$ tongue atlas was used to study subject-to subject, subject-to-atlas, and male-to-female morphometric variation. Preliminary results show the major difference among female subjects before and after the TPS analysis is in the area of tongue dorsum that is close to the velum and epiglottis, respectively. However, the major difference among male subjects is in the areas of tongue tip and body regardless of TPS analysis. In summary, our preliminary results imply that the 3D tongue atlas of female subjects show less subject-to-subject difference.

1pSCc26. Neural correlates on Chinese speech production: an fMRI study. Chao-Min Wu (Dept. Electrical Engineering, National Central University, \#300, Chung-Da Rd., 320 Chung-Li, Taiwan, wucm@ee.ncu.edu.tw), Yi-Syuan Lin (Dept. Electrical Engineering, National Central University, \#300, Chung-Da Rd., 320 Chung-Li, Taiwan, kenshin5718@yahoo.com.tw)

The aim of this study is to use functional magnetic resonance imaging (fMRI) method to find neural correlates on Chinese speech production. Three young normal adults (mean age $24 \mathrm{yrs}$ ) produced four tone Chinese characters "ma1, ma2, ma3, and ma4" in four ways: (A) normal speech, (B) mouthing speech, (C) unarticulated speech, (D) internal speech, and "ma1, ma1, ma1, and ma1" for the condition (E) normal speech. The fMRI results showed that the areas associated with the control of breathing for speech, vocalization, and hearing were the sensorimotor cortex, supplementary motor area, the superior temporal gyrus, the cerebellum, parietal association area, and the lingual gyrus. The areas associated with articulation were the sensorimotor cortex, the cerebellum, the inferior frontal gyrus, anterior and posterior cingulate gyrus, and the precuneus. The areas associated with tone variation were found in bilateral basal ganglia, hippocampal formation, and parietal association area, and the left pre-motor area, middle occipital gyrus, and the right cerebellum, superior temporal gyrus, and midbrain. Neural correlates of speech production and Chinese tone were all related to the cerebellum. In summary, our preliminary results showed the cerebellum plays an important role on Chinese speech production.

1pSCc27. Differences in the development of speech segmentation abilities in two French dialects. Thierry Nazzi (CNRS - Université Paris Descartes, 45 rue des Saints-Pères, 75006 Paris, France, thierry.nazzi @ univ-paris5.fr), Karima Mersad (CNRS - Université Paris Descartes, 45 rue des Saints-Pères, 75006 Paris, France, crocnotekary@yahoo.fr),Galina Iakimova (CNRS - Université Paris Descartes, 45 rue des Saints-Pères, 75006 Paris, France, galina.iakimova@free.fr), Megha Sundara (UCLA Department of Linguistics, 3125 Campbell Hall, Los Angeles, CA 900951543, USA, megha.sundara@humnet.ucla.edu), Linda Polka (McGill University, School of Communication Sciences and Disorders, Beatty Hall, 1266 Pine Avenue West, Montreal, QC H3G 1A8, Canada, linda.polka @ mcgill.ca)

Speech segmentation skills develop in infancy and are influenced by many phonological properties of the native language and, in particular, the prosodic structure of the infant's native language. Studies using the HPP task show that American English infants appear to favor a stress-based procedure (Jusczyk et al., 1999) whereas Parisian French infants favor a syllable-based procedure (Nazzi et al., 2006), in line with a prosodic-based bootstrapping account of segmentation abilities (Nazzi et al., 1998). However, in a study using different stimuli, Polka and Sundara (2003) found results that might suggest a developmental trajectory for Canadian French infants that does not rely on syllable-based segmentation. Given that the stimuli used in both studies on French were different, both research teams tested their infant populations (at 8, 12, and 16 months of age) with the stimuli originally used by the other team The results suggest a complex interaction between specific stimuli and infants' native dialect, thus providing for the first time data suggesting that word segmentation is dialect specific. We will discuss how these findings, together with acoustic analyses, show that early word segmentation is influenced by linguistic (rhythmic/syllabic, dialectal) prosodic cues, extra-linguistic (speech style) prosodic cues, and distributional cues.

1pSCc28. Effect of consonant gemination on vowel coarticulation. Maria-Gabriella Di Benedetto (University of Rome, Infocom Dept. Via Eudossiana 18, 00184 Rome, Italy, gaby@acts.ing.uniroma1.it), Daniele Domenicali (University of Rome, Infocom Dept. Via Eudossiana 18, 00184 Rome, Italy, dome@newyork.ing.uniroma1.it)

As well known V1CV2 structures are typically characterized by two different phenomena: the first vowel V1 spectral properties are affected by the presence and nature of the second vowel V2 (anticipatory effect), and V2 is influenced by the presence of the first one (cross-over effect). The intensity of anticipatory and the cross-over effects are language dependent and can vary significantly. Both effects have been observed in the past to be present in the Italian language through the analysis of VCV nonsense words. The Italian language has however a unique property, that is distinctive consonant gemination, i.e. consonant length is phonemic; two different words may thus differ by the sole presence or absence of gemination in one of this consonants. The Italian language offers therefore the ideal framework for analyzing the effect of gemination on across-consonant coarticulation. The object of this work is to analyze whether and how the presence of gemination may affect the acoustic properties of vowels in V1CCV2 words. First results of acoustic analysis of V1CV2 as well as their V1CCV2 counterparts, spoken by Italian native speakers, tend to show that gemination may have an effect on across-consonant coarticulation, with specific peculiarities for anticipatory vs. cross-over effects.

1pSCc29. Enhancement of voiced and voiceless stop categories in infant-directed speech to 11-month-old infants. Gina C. Cardillo (University of Washington, Dept. of Speech \& Hearing Sciences, and Institute for Learning \& Brain Sciences, Box 357988, Seattle, WA 98195, USA, ginacc@u.washington.edu), Jennifer R. Perry (University of Washington, Dept. of Speech \& Hearing Sciences, and Institute for Learning \& Brain Sciences, Box 357988, Seattle, WA 98195, USA, jperry3@u.washington.edu), Patricia K. Kuhl (University of Washington, Dept. of Speech \& Hearing Sciences, and Institute for Learning \& Brain Sciences, Box 357988, Seattle, WA 98195, USA, pkkuhl@u.washington .edu)

It has been proposed that infant-directed speech (IDS) increases the discriminability of phonetic categories by exaggerating the acoustic differences between phonetic units (Kuhl et al., 1997, Science). However, reports show conflicting results on whether this principle holds for consonants. The current study measured English /p/ /b/ /t/ /d/ in both IDS and adult-directed 
speech (ADS) from connected speech in 27 mothers addressing their 11month-old infants. Findings supported the exaggeration hypothesis. The mean VOT of /p/ was longer in IDS than ADS, and approached significance for $/ \mathrm{t} /$. The mean VOT difference between $/ \mathrm{p} /$ and $/ \mathrm{b} /$ was greater in IDS than in ADS. Similarly, when phonemes were grouped into voiceless and voiced categories, the difference between categories was significantly greater in IDS than ADS. We will discuss ways to measure AD-ID differences. [Work supported by NIH HD37954.]

1pSCc30. Speaker-specific patterns of token-to-token variability. Christine Mooshammer (Haskins lab and MIT Research Lab of Electronics, 300 George Street, Suite 900, New Haven, CT 06511, USA, tine@ haskins.yale.edu), Pascal Perrier (ICP/GIPSA-lab, INPG, 46 Avenue Félix Viallet, 38031 Grenoble Cedex 01, France, Pascal.Perrier@gipsa-lab.inpg.fr), Susanne Fuchs (ZAS, Schuetzenstr. 18, 10117 Berlin, Germany, fuchs@zas.gwz-berlin.de)

One explanation for token-to-token variability is neural noise corrupting the motor commands. In a previous study using a two-dimensional tongue model, adding neural noise to motor commands was able to account for shapes and orientations of the dispersion ellipses of tongue positions for non-high vowels only. It was concluded that additional sources shaping lingual variability patterns of vowels should be examined: (a) stabilization of the tongue by lateral palate contact, (b) palate shape and (c) perceptual constraints due to the crowdedness of the vowel space. Variability patterns of three speakers of German were analyzed by means of EMMA, EPG, and formant frequencies. Only the most variable speaker used lingual-palatal contact to reduce lingual variability for high front vowels. The other two speakers achieved articulatory vowel targets in a very precise manner. A flat palate shape can explain the limited lingual variability of one of these two. Only for this speaker did the acoustic distance between vowel targets significantly limit acoustical variability with smaller Bark ellipsoid volumes in regions with closer neighbors. For the other precise speaker, neither palate shape nor perceptual demands were relevant. We conclude that the relative importance of the factors analyzed varies across speakers.

1pSCc31. Neural mechanism for understanding speakers' intentions: developmental analyses of children with and without communication disorders. Satoshi Imaizumi (The Prefectural Univ. of Hiroshima, 1-1 Gakuen-machi, 723-0053 Mihara, Japan, imaizumi@pu-hiroshima.ac.jp)

Ability to understand speakers' intentions through linguistic contents and affective prosody is examined for children between 5 and 12 years-old with and without communication disorders. Four types of spoken short phrases, expressing admiration, sarcasm, blame, and humor or joke, were presented. For each stimulus, the subjects were asked to choose between two cards, one with a written word "praise" and another with "no praise" accompanied by corresponding drawings. For children without any communication disorders, the percentage of the correct judgment of speaker intentions was high and stable for admiration and blame phrases which have congruent linguistic and affective valences. It was significantly low for 6-year-old children and increased with age for the sarcastic or joking phrases which have incongruent linguistic and affective valences. The percent correct was significantly lower for autistic children than normally developing children particularly for the phrases with incongruent valences. Although a significant difference was found between children with severe- and mild-hearing impairment, no significant difference was found in the percent correct between the congruent and incongruent phrases for them. Based on these results together with brain activation analyses using fMRI (Imaizumi et al., 2004, 2006), a model of neural mechanism for understanding speakers' intentions is discussed.

1pSCc32. Perception of two English front vowels by L1 Dutch speakers with different regional accents. Paola Escudero (University of Amsterdam, Institute of Phonetic Sciences, Spuistraat 210, 1012 VT Amsterdam,
Netherlands, P.R.EscuderoNeyra@uva.nl),Ellen Simon(Ghent University, English Department, Rozier 44, 9000 Ghent, Belgium, Ellen.Simon@UGent .be)

This paper reports the results of a categorization task of the English vowels "epsilon" and "ash" by native speakers of Dutch. Dutch has only one member of the contrast ("epsilon"), the acoustic realization of which is subject to considerable regional variation (Adank et al., 2007). Previous research has shown that that there is asymmetrical lexical competition between English words containing "epsilon" and "ash" for native speakers of Dutch (Weber \& Cutler, 2004; Escudero et al., in press). Weber \& Cutler (2004) argue that "epsilon" is the dominant category for native speakers of Dutch, because it is phonetically closer to the L1 Dutch sound. The present study investigates whether the dominant category may vary depending on the speakers' L1 dialect by examining how two groups of Dutch-speaking participants from different dialect regions (the Randstad region in the Netherlands and East-Flanders in Belgium) perceive the English vowels "epsilon" and "ash." Preliminary results indicate that participants from these two area's do indeed categorize English "epsilon" and "ash" differently as the result of the acoustic properties of their L1 vowel "epsilon" (F1, F2 and duration).

1pSCc33. Motor equivalent strategies in the production of $/ \mathbf{u} /$ in perturbed speech. Jana Brunner (Humboldt-Universität Berlin/Gipsa-lab, INP Grenoble, ZAS, Schützenstr. 18, 10117 Berlin, Germany, brunner@zas.gwz-berlin.de), Phil Hoole (Institut für Phonetik und Sprachverarbeitung, Schellingstr. 3, 80799 München, Germany, hoole@phonetik.uni-muenchen.de), Pascal Perrier (ICP/GIPSA-lab, INPG, 46 Avenue Félix Viallet, 38031 Grenoble Cedex 01, France, Pascal.Perrier @ gipsa-lab.inpg.fr)

Several articulatory strategies are available during the production of $/ \mathrm{u} /$, all resulting in a similar acoustic output. For example, speakers can protrude the lips and compensate for that by widening the velar constriction. This study investigates whether speakers use this relation under perturbation. Five speakers were provided with palatal prostheses which were worn for two weeks. Speakers were instructed to make a serious attempt to produce normal speech. Their speech was recorded via EMA and acoustics several times over the adaptation period. Formant values of / $\mathrm{u}$-productions were measured. Velar constriction width and lip protrusion were estimated. For four speakers a correlation between constriction width and lip protrusion was found. A correlation between lip protrusion and F1 or F2 was rare and no correlation occurred between constriction size and either of the formants. The acoustic output was thus constant. The results show that under perturbation speakers use motor equivalent strategies. The correlation between constriction size and lip protrusion is stronger than in studies investigating unperturbed speech (e.g., Perkell et al., 1993). This could be because under perturbation speakers are inclined to try out several strategies in order to reach the acoustic target and the co-variability might thus be greater.

1pSCc34. Using sets of combs to control pitch estimation errors. Jean-Sylvain Lienard (LIMSI-CNRS, BP133, 91403 Orsay Cedex, France, jean-sylvain.lienard@limsi.fr), Claude Barras (LIMSI-CNRS, BP133, 91403 Orsay Cedex, France, claude.barras@limsi.fr), Francois Signol (LIMSI-CNRS, BP133, 91403 Orsay Cedex, France, francois.signol @ limsi.fr)

We analyze the errors of a Pitch Estimation Algorithm using the Pitch Function (response of the periodicity estimator as a function of the frequency parameter $F_{c}$ ). The estimator's maximum response for a single signal of fundamental frequency $F_{0}$ is expected to occur for $F_{c}=F_{0}$. Actually the pitch function exhibits many secondary peaks which occasionally cause the errors. When several signals are mixed, secondary peaks of a signal may take over the main peak of the other. By taking as periodicity estimator the correlation of the spectrum module with a uniform infinite spectral comb of fundamental frequency $F_{c}$ we show that each peak corresponds to a particular value of the ratio $F_{c} / F_{0}=p / q$ ( $p$ and $q$ integers $\geqslant 1$ ). It follows that some secondary peaks can be canceled either by augmenting the comb with intermediary negative teeth, or by setting to zero some of its teeth. These modified combs can be viewed as linear combinations of uniform combs of different $F_{c}$ s. The present study aims at precisely defining and combining the modified combs so that the main peaks of the new Pitch Function reliably 
indicate the $F_{0} \mathrm{~s}$ of the components. Examples are given on mixtures of voiced segments extracted from natural speech.

1pSCc35. Articulatory exaggerations during infant directed speech. Jordan R. Green (University of Nebraska, 3180 Barkley Center, Lincoln, NE 68583-0738, USA, jgreen4@unl.edu), Ignatius Nip (San Diego State University, 5500 Campanile Dr., San Diego, CA 92182-1518, USA, inip@mail.sdsu.edu), Erin Wilson (Waisman Center, 1500 Highland Avenue, Madison, WI 53705-2280, USA, emhillman@wisc.edu), Antje Mefferd (University of Nebraska, $318 \mathrm{O}$ Barkley Center, Lincoln, NE 68583-0738, USA, amefferd@unlserve.unl.edu)

Although a growing body of literature suggests a principal role for observational learning in the acquisition of speech, the facial movements of infant directed speech (IDS) have rarely been studied. Identifying both the auditory and visual features of IDS is an essential step toward understanding the contribution of environmental stimulation to the development of speech and language. The current project uses three-dimensional motion capture technology to describe how parents modify their articulatory movements when communicating with their infants. The following three experimental questions will be addressed: (1) Do parents exaggerate articulatory gestures during IDS?; (2) If so, are there individual differences among parents in the degree of IDS?; and (3) Is there a strong association between acoustic and movement characteristics of IDS? Mouth movements were recorded from twenty-four mothers while speaking to their infants and to an unfamiliar adult. Mouth shapes during four target vowels were measured for differences in maximum vertical separation, maximum horizontal spread, and duration across speaking conditions. The result indicated that the majority of mothers exaggerated their lip movements when speaking to their infants. Speculation is made regarding the potential role of articulatory exaggerations in early speech learning.

1pSCc36. Evaluation of the Pitch Estimation Algorithms in the monopitch and multipitch cases. Francois Signol (LIMSI-CNRS, BP133, 91403 Orsay Cedex, France, francois.signol@limsi.fr), Claude Barras (LIMSI-CNRS, BP133, 91403 Orsay Cedex, France, claude.barras@limsi.fr), Jean-Sylvain Lienard (LIMSI-CNRS, BP133, 91403 Orsay Cedex, France, jean-sylvain.lienard@limsi.fr)

Reliably tracking the fundamental frequency $F_{0}$ of the components is an important step in the separation of superimposed speech signals. Several Pitch Estimation Algorithms are potentially usable and a rigorous evaluation method is needed in order to compare them. However, even in the monopitch case, many variations between them render such a comparison difficult

. The extent of the $F_{0} \min -F_{0} \max$ interval, the use of a priori information on the whole sequence or database, and above all the arbitrary setting of the voicing threshold, yield large differences in the results. These biases can be removed by setting the $F_{0}$ bounds to fixed values acceptable for many voices, by proceeding with the evaluation on a strictly frame-to-frame basis, and by fixing the voicing threshold in order to get an equal error rate for overvoiced and undervoiced frames. In the multipitch case any frame may exhibit 0,1 , or 2 valid voicings according to the coincidence between the voiced and unvoiced parts of both signals. This problem is treated by defining a similarity measure linking the PEA's hypotheses to the pitch values of the isolated signals. The proposed methodology is applied to several PEAs, using the Keele database in monopitch and multipich setups.

1pSCc37. An acoustic investigation of the Swedish child's acquisition of obstruent place of articulation. Jan Van Doorn (Umeå University, Department of Clinical Sciences, SE90187 Umeå, Sweden, jan.vandoorn@logopedi.umu.se), Fredrik Karlsson (Umeå University, Department of Language Studies, SE90187 Umeå, Sweden, fredrik.karlsson@ling.umu.se), Kirk P. Sullivan (Umeå University, Department of Language Studies, SE90187 Umeå, Sweden, kirk.sullivan@ling .umu.se)

Speech produced by children in the initial stages of development does generally not uphold as many phonetic distinctions as speech sounds produced by adults. A child's productions of different target words may therefore have similar acoustic properties and result in homonyms being perceived by the adult observer. This study presents a longitudional investigation into the development of place of articulation from nondistinctive to distinctive productions in word-initial obstruents produced by 22 Swedish children (aged 18 - 48 months). The data was collected through monthly recordings, approximatelly one year per child. The acoustic correlates analysed were spectral diffuseness, spectral skewness and spectral tilt for plosives and spectral skewness, spectral kurtosis, spectral variance and F2 onset frequency for fricatives. The results show a developmental trend in spectral skewness that is indicative of a increasing number of acquired phonetic contrasts. Spectral tilt change, F2 onset frequency, spectral mean and spectral variance provide evicence of within-category refinement wich is argued to be caused primarilly by advancements in motor control.

1pSCc38. Nonlinear dynamics of speech categorization: critical slowing down and critical fluctuations. Leonardo Lancia (LPL, Aix-Marseille Université \& CNRS, 29, avenue Robert Schuman, 13100 Aix-en-Provence, France, leonardo.lancia@lpl-aix.fr), Noel Nguyen (LPL, Aix-Marseille Université \& CNRS, 29, avenue Robert Schuman, 13100 Aix-en-Provence, France, Noel.Nguyen@1pl-aix.fr), Betty Tuller (National Science Foundation, 4201, Wilson Boulevard, Arlington, VA 22230, USA, tuller@ccs.fau .edu)

The dynamical system view of speech perception assumes that speech categorization depends on both the acoustic properties of the incoming sound and the state of the perceptual system (indexed by the subject's responses to preceding sounds). Tuller and colleagues [1] systematically manipulated the order of presentation of stimuli from a "say"-"stay" continuum in a binary-choice word identification task. Listeners' responses were modeled using a nonlinear dynamical system whose point attractors were associated with perceptual categories. In the present study, we asked French speakers to categorize stimuli on a continuum from "cèpe" /sep/ to "steppe" /step/. Our results provide support for two yet untested predictions from the model: the occurrence of a) critical slowing down and b) critical fluctuations . Critical slowing down relates to the increase in relaxation time that occurs as the system approaches points of instability. Critical fluctuations refer to the system's increased sensitivity to random fluctuations near the points of instability. [Work supported by the CNRS \& French Ministry of Research, and by NSF.] [1] P. Case, B. Tuller, D. Mingzhou, and J.A.S. Kelso. Evaluation of a dynamical model of speech categorization. Perception and Psychophysics, 57:977-988 (1995).

1pSCc39. The effects of vowel identification training on sentence-level speech recognition. Carolyn Richie (Communication Disorders, Butler University, 4600 Sunset Ave., Indianapolis, IN 46208, USA, crichie@butler .edu)

Recent work examined the effects of a novel computer-based approach to speech recognition training involving vowel identification [C. Richie, J. Acoust. Soc. Am. 121, 3045 (2007)]. When vowel identification training was done under auditory-visual conditions with noise participants demonstrated modest improvements in sentence recognition, and when training was done under visual-only conditions participants failed to show improvements in sentence recognition. However, sentence recognition was assessed for the CID Everyday Sentences in terms of words correctly recognized. Improvements in speech recognition may have occurred following training on a level lower than the word, and gone unrecognized given this scoring method. The purpose of the present study is to determine whether vowel identification training leads to improvements in speech recognition on a test of sentence recognition, as measured by correct identification of consonants and vowels. These findings have implications for sentence recognition test scoring methods. The role of acoustics in improving speech recognition will also be discussed. (Supported by Butler University HAC grant \#027096).

1pSCc40. An acoustic study on voiceless retroflex and dental sibilants of young native Min speakers in Taiwan. Yu-Ying Chuang (Graduate Institute of Linguistics, Grad. Inst. of Linguistics, National Taiwan Univ., 1, 
Roosevelt Rd. Sec. 4, 106 Taipei, Taiwan, r95142001@ntu.edu.tw),Janice Fon (Grad. Inst. of Linguistics, National Taiwan Univ., 1, Roosevelt Rd. Sec. 4, 106 Taipei, Taiwan, jfon@ntu.edu.tw)

The Mandarin spoken in Taiwan is influenced by Min, a Southern Chinese dialect, in many perspectives. Due to the absence of retroflex sibilants in Min, previous studies claimed that retroflex sibilants are replaced by their dental counterparts. Recent studies, however, showed that retroflex and dental sibilants are actually distinguished. Such contradictory result was due to the fact that different speaker groups were targeted at - one on older generation, acquiring Min as L1, whereas the other on younger generation, acquiring Min as L2. Since age and the acquisition order of Min were involved, it was unknown which factor affected the realization of retroflex and dental sibilants more. To clarify this point, we targeted at the younger generation that acquired Min as L1. Acoustic measurements were taken on all sibilants. Results showed that retroflex and dental sibilants had distinctive realization, suggesting age factor to be more determent. Gender effect was found significant, in which females showed a greater distinction than males. Moreover, retroflex sibilants pronounced in syllables were significantly different from those in sentences. Future studies could be done on the other group - older generation, acquiring Min as L2. Also, whether the frequency of using Min plays a role awaits further exploration.

1pSCc41. A comparison of native speaker and American adult learner Vietnamese lexical tones. Allison Blodgett (University of Maryland College Park, Center for Advanced Study of Language, 7005 52nd Ave, College Park, MD 20742, USA, ablodgett@ casl.umd.edu), Jessica Bauman (University of Maryland College Park, Center for Advanced Study of Language, 7005 52nd Ave, College Park, MD 20742, USA, jbauman@hesp.umd.edu), Anita Bowles (University of Maryland College Park, Center for Advanced Study of Language, 7005 52nd Ave, College Park, MD 20742, USA, abowles@casl.umd.edu), Lykara Charters (University of Maryland College Park, Center for Advanced Study of Language, 7005 52nd Ave, College Park, MD 20742, USA, lykarac@mac.com), Anton Rytting (University of Maryland College Park, Center for Advanced Study of Language, 7005 52nd Ave, College Park, MD 20742, USA, crytting@casl.umd.edu), Jessica Shamoo (University of Maryland College Park, Center for Advanced Study of Language, 7005 52nd Ave, College Park, MD 20742, USA, jshamoo@casl.umd.edu), Matthew Winn (University of Maryland College Park, Department of Hearing \& Speech Sciences, 0100 Lefrak Hall, College Park, MD 20742, USA, mwinn@hesp.umd.edu)

This study introduces a modification of the traditional carrier sentence elicitation task, provides new native speaker data regarding aspects of pitch and voice quality in Vietnamese lexical tones, and provides an empirical description of non-native speaker errors in tone production. Participants produce a series of three-word utterances in response to target words that appear individually on a computer screen in one of four colors. In this way, participants actively describe a changing event, while critical components remain constant: the identity of the word preceding the target, the target position (utterance medial), and the target's focus structure. Native speaker results are consistent with existing descriptions of fundamental frequency contours and creakiness. In addition, they provide evidence supporting claims that breathiness can occur in two different tones: the falling tone (huyen) and curve tone (hoi). Non-native speaker results suggest that adult learners struggle with voice quality distinctions and pitch shape.

1pSCc42. Lip protrusion and constriction in anticipation in English and French. Aude Noiray (Haskins Laboratories, 300 George St, New Haven, CT 06511, USA, aude.noiray@haskins.yale.edu), Lucie Ménard (Département de linguistique et de didactique des langues, Université du Québec à Montréal, Case postale 8888, succursale Centre-ville, Montréal, QC H3C 3P8, Canada, menard.lucie@uqam.ca), Marie-Agnès Cathiard (Centre de Recherche sur l'Imaginaire (CRI), Université Stendhal, Grenoble 3, BP 25, 38040 Grenoble cedex 9, France, Marie-Agnes
.Cathiard@u-grenoble3.fr),Christian Abry(Centre de Recherche sur l'Imaginaire (CRI), Université Stendhal, Grenoble 3, BP 25, 38040 Grenoble cedex 9, France, christian.abru@u-grenoble3.fr)

Though lip constriction is acoustically the most robust parameter for rounding, most studies on anticipatory coarticulation relied on the investigation of protrusion via EMG, optoelectrical systems or audiovisual processing. These various methods produced inconsistent anticipatory profiles across studies or even speakers. The Movement Expansion Model (MEM) of Abry and Lallouache has explained vocalic anticipatory coarticulation in four French adults and eight children via lip constriction, showing idiosyncratic behaviours, i.e. personal regression slopes but with a lawful variability validated by high correlation coefficients. In order to describe global anticipatory movement, the current study included measures of both protrusion and constriction over time. Four American English and four $\mathrm{Ca}-$ nadian French adults were audio visually recorded, uttering $[\mathrm{iC}[\mathrm{n}] \mathrm{u}]$ sequences, in which $\mathrm{C}[\mathrm{n}]$ corresponded to a varying number of intervocalic consonants. Lip protrusion was measured via an Optotrak system, and constriction via a Lip-Shape Tracker (from ICP Grenoble). We found that when tested on lip area control, English speakers joined French speakers in a regular MEM temporal pattern, displaying their own anticipatory behaviour, with personal regression slope or expansion coefficient.

1pSCc43. The contribution of vowel production to the intelligibility and accentedness of nonnative speech. Jessica E. Alexander (Dept. of Psychology, Emory University, 532 Kilgo Cir, Atlanta, GA 30322, USA, jessica.alexander@emory.edu), Sabrina K. Sidaras (Dept. of Psychology, Emory University, 532 Kilgo Cir, Atlanta, GA 30322, USA, ssidara@emory.edu), Lynne C. Nygaard (Dept. of Psychology, Emory University, 532 Kilgo Cir, Atlanta, GA 30322, USA, lnygaar@emory.edu)

The present study investigated the extent to which the perception of accentedness and intelligibility in nonnative speech depends on the specific acoustic realization of vowels produced by nonnative speakers. Twelve native speakers of Korean were recorded producing both word- and sentencelength English utterances. Acoustic analyses, including measures of vowel duration and formant frequencies, were conducted on tokens of individual English vowels identified from the word-length utterances. Vowel tokens were selected from a variety of phonetic contexts and were compared to productions of the same vowels produced in the same contexts by native English speakers. A separate set of native English-speaking listeners judged accentedness and intelligibility from the sentence-length utterances. The results showed that both the duration and spectral characteristics of Koreanaccented vowels were related to overall sentence intelligibility and ratings of perceived accentedness. These findings suggest that the detailed acoustic realization of the temporal and spectral structure of non-native vowels contributes to global measures of perceived accentedness and overall intelligibility.

1pSCc44. Word-initial voiceless geminate stops: production and perception. Rachid Ridouane (Laboratoire de Phonétique et PhonologieUMR7018 CNRS/Sorbonne Nouvelle, 19 rue des Bernardins, 75005 Paris, France, rachid.ridouane@univ-paris3.fr), Pierre Halle (Laboratoire de Phonétique et Phonologie-UMR7018 CNRS/Sorbonne Nouvelle, 19 rue des Bernardins, 75005 Paris, France, pierre.halle@univ-paris5.fr)

Quantity contrasts (i.e., singleton vs geminate consonants, short vs long vowels) are common in the languages of the world. Yet, most singletongeminate contrasts occur in word-medial position. Languages that allow gemination in other than word-medial position (word-initially or wordfinally) are quite few. Even less frequent is the occurrence in the languages of the world of word-initial voiceless geminate stops. Pattani Malay (Abramson, 1986), Turgovian Swiss German (Kraehenmann, 2003), Tashlhiyt Berber (Ridouane, 2007), are the only documented languages that allow singleton-geminate contrasts word-initially for all native consonants, including both voiced and voiceless plosives. In this work we deal with wordinitial voiceless geminate stops in Tashlhiyt Berber and present results from 3 experiments: 1) a production study including acoustic and electropalatographic measurements 2) a native-listener experiment with labelling and discrimination, and 3) a cross-language perceptual experiment, with listeners of 
two languages differing in germination: Italian (in which gemination only occurs word-medially) and French (in which gemination only occurs between words in continuous speech).

1pSCc45. Inter-speaker variability and the articulatory-acoustic relations in German and English /sh/. Susanne Fuchs (ZAS, Schuetzenstr. 18, 10117 Berlin, Germany, fuchs@zas .gwz-berlin.de), Martine Toda (CNRS \& ENST, 46, rue Barrault, 75634 Paris, France, martinetoda@yahoo.co.jp)

The aim of this study is to investigate speaker-specific articulatoryacoustic relations and their potential causes in the realisation of $/ \mathrm{sh} /$. By means of electropalatographic and acoustic data for 12 German and 12 English native speakers (6 males and 6 females for each language), we obtained the following results: 1) relatively invariant $\mathrm{COG}$ values of the frication noise among the subjects, 2) huge inter-speaker variability in the articulatory place of articulation, especially for the males. We suggest that in the realisation of $/ \mathrm{sh} /$ motor equivalence principles can be used speakerdependently. In particular, the length of palatal channel, the size of the front cavity (including its sublingual portion) as well as lip protrusion are the relevant articulatory parameters involved in the adjustment of the frication noise. Speaker-specific articulatory strategies will be discussed in the light of the formant transition characteristics, palatal morphology, and the prototypical articulatory patterns will be illustrated by means of acoustic modelling.

1pSCc46. Pitfalls of spectrogram readings of flaps. Minjung Son (Yale University and Haskins Labs, 300 George St. Suite 900, New Haven, CT 06511, USA, son@haskins.yale.edu)

Several acoustic studies have revealed stable characteristics of the speech signal for rhotics, whereas articulatory studies have found different articulatory strategies across speakers for producing these segments (Ladefoged and Maddison, 1996; Tiede et al., 2004). However, no study has showed that the phonologically reduced flap gesture, which is argued to be a lateral in the underlying representation, can sometimes fail to be reflected in the acoustic correlates of an articulatory event. Using electromagnetic midsagittal articulometry (Perkell et al., 1992), a case study has been conducted for Korean. Productions of two Seoul-Korean speakers were collected using real words in the within-word condition. The subjects read target words within a single accentual phrase, as in Jun (1993), and repeated eight times. Each target word (/ili/ and /ala/) appeared in its own distinct natural sentence at two speech rates (fast and comfortable). A total of 32 tokens were available for analysis. Tongue tip gestures were examined simultaneously with spectrograms. Results from one speaker indicated that sometimes no acoustic correlates of the tongue tip gesture for a flap were present (6.3\% of productions) although articulation was obviously produced [Work supported by NIH DC00403.]

1pSCc47. Clicks with noisy accompaniment in West !Xoon. Sven Grawunder (Max Planck Institute for Evolutionary Anthropology, Deutscher Platz 6, 04103 Leipzig, Germany, grawunder@eva .mpg.de), Christfried Naumann (Max Planck Institute for Evolutionary Anthropology, Deutscher Platz 6, 04103 Leipzig, Germany, naumann@eva .mpg.de)

The current study is concerned with ingressive stops (clicks) with a noisy accompaniment exemplified by data from West !Xoon, a Taa variety ("!Xóõ", Tuu/Southern Khoisan) spoken in Namibia. Within the obstruent system of West !Xoon, we describe (1) aspirated clicks, (2) clicks followed by a laryngeal fricative, (3) clicks with an aspirated uvular accompaniment, and (4) clicks followed by a uvular fricative. In previous analysis for the variety of East !Xoon (Traill and Ladefoged, 1994), (2) were analysed as series of "delayed aspiration", while the contrast between (1) and (3) was ignored. A novelty discovery are prenasalized "voiced" clicks followed by [h], the voiced counterparts of clicks with "delayed aspiration", which are probably absent in East !Xoon. We present an acoustic analysis of alveolar and palatal clicks based on field data of 6 speakers ( 3 male $/ 3$ female), where for each click type plus noise accompaniment three tokens in \#CV context had been recorded repeatedly. As descriptive parameters served durational measures of burst length, cluster length, length of aspiration delay, and spectral measures of burst and friction parts. In opposite to the previous distinction our findings suggest a clear 3-way distinction of clicks (1-3) with noisy accompaniment.

1pSCc48. Blind source separation and directional audio synthesis for binaural auralization of multiple sound sources using microphone array recordings. Banu Gunel (I-Lab Multimedia and DSP Research Group, Centre for Communication Systems Research, University of Surrey, GU2 7XH_Guildford, UK, B.Gunel@surrey.ac.uk), Huseyin Hacihabiboglu (I-Lab Multimedia and DSP Research Group, Centre for Communication Systems Research, University of Surrey, GU2 7XH Guildford, UK, h.hacihabiboglu@surrey.ac.uk), Ahmet Kondoz (I-Lab Multimedia and DSP Research Group, Centre for Communication Systems Research, University of Surrey, GU2 7XH Guildford, UK, A.Kondoz@surrey.ac.uk)

Microphone array signal processing techniques are extensively used for sound source localisation, acoustical characterisation and sound source separation, which are related to audio analysis. However, the use of microphone arrays for auralisation, which is generally related to synthesis, has been limited so far. This paper proposes a method for binaural auralisation of multiple sound sources based on blind source separation (BSS) and binaural audio synthesis. A BSS algorithm is introduced that exploits the intensity vector directions in order to generate directional signals. The directional signals are then used in the synthesis of binaural recordings using head related transfer functions. The synthesised recordings subsume the indirect information about the auditory environment conveying the source positions and the acoustics similar to dummy head recordings. Test recordings were made with a compact microphone array and a dummy head in different indoor environments. Subjective listening tests were performed to compare the synthesized recordings with the actual ones for their performance in providing presence, spaciousness and envelopment.

1pSCc49. Bilinguals' and monolinguals' reaction-time in two language contexts: evidence for the double phonetic representation in bilinguals. Adrian Garcia-Sierra (University of Washington, Institute for Learning and Brain Sciences, Box 357988, Seattle, WA WA 98195-7988, USA, gasa@u.washington.edu), Craig Champlin (University of Texas, 1 University Station A1100, Austin, TX 78712-0114, USA, champlin@austin.utexas .edu)

The time between the presentation of a stimulus and the response given to it is occupied by a series of mental operations reflecting the decision perse. Reaction-time (RT) can be thought as the duration of the mental operations behind making a decision. Small RT values involve less mental operations than larger RT values. In speech categorization tasks, sounds with clear phonetic membership produce small RT values, and ambiguous speech sounds produce large RT values. In the present investigation a Go-No-Go task was implemented to assess RT from Spanish-English bilinguals $(\mathrm{N}=27)$ and English monolinguals $(\mathrm{N}=27)$ in two language contexts. Participants were asked to identify the speech sound "ta" from a 10 token speech continuum varying from -20 to $+25 \mathrm{~ms}$ of VOT. It was expected that bilinguals, but not monolinguals, would perceive +VOT stimuli as clear representations of ' $t$ ' (faster RT) in the Spanish language context, but as ambiguous representations of ' $t$ ' in the English language context. The results confirmed the expectations. That is, language contexts affected the speed by which mental operations were produced in bilinguals. This outcome is in agreement with the idea that bilinguals possess a double phonemic representation. Challenges and limitations of the present study are discussed.

1pSCc50. Is there a sensitive period for representation of phoneme sequences? Mary Anne Welton (University of Prince Edward Island, 550 University Ave, Charlottetown, PE C1A 4P3, Canada, mwelton@upei.ca), Annabel J. Cohen (University of Prince Edward Island, 550 University Ave, Charlottetown, PE C1A 4P3, Canada, acohen@upei.ca)

A key issue in development of speech perception and production concerns the possible existence of an early sensitive period that facilitates language acquisition. Spoken language acquisition entails representation of grammatical sequences of phonemes. In a two-phase experiment, 120 participants in three age groups (pre-adolescents, adolescents, young adults) were presented with 64 words, 16 each of English, English pronounceable 
non-words, Spanish, and Mandarin. The task was to determine if the word was English. All age groups performed well, indicating that the words had been attended. A subsequent surprise recognition phase presented 64 words again, one half of which were old and one half new. Participants judged whether each word had previously been presented. Here performance overall was poorest for Spanish and Mandarin, and children performed relatively more poorly on English non-words as compared to the older groups, but showed no age-relative disadvantage for Chinese. Arguing that evidence for a sensitive period arises if children perform relatively better than older participants on foreign words as compared to English words or English nonwords, the recognition results are consistent with the proposal of a sensitive period for phoneme sequence representation. The paradigm provides a new way of examining the sensitive period hypothesis. (supported by NSERC)

1pSCc51. A detailed biomechanical finite element tongue model. Reiner F. Wilhelms-Tricarico (Rewtnode, 27 Old Bay Road, Belchertown, MA 01007, USA, rewtnode@gmail.com), Maureen Stone (Vocal Tract Visualization Lab, Depts. of Biomedical Sciences and Orthodontics, University of Maryland Dental School, 650 W. Baltimore St., Baltimore, MD 21201, USA, mstone@umaryland.edu), Mark Carlson (MSC.Software Professional Services, 4332 Brookside Avenue, Minneapolis, MN 55436, USA, mark.carlson@mscsoftware.com), Paul Buscemi (Restore Medical, Inc, 2800 Patton Rd, Roseville, MN 55113, USA, pbuscemi@ restoremedical .com)

For research on surgical modifications of the tongue, e.g., implants for treatment of sleep apnea, we built a detailed finite element model of the human tongue, to experimentally estimate and investigate muscle activations corresponding to observed movement data, and to study possible effects of tissue modifications. Most of the extrinsic and all intrinsic muscles of the tongue are represented as parametrized volumes within the tongue, and muscle fiber directions are specified by a tangent in curvilinear coordinate systems of parametric solids that overlay each muscle region. Fiber regions of different muscles may overlap, for example, for the transverse and vertical intrinsic muscles. Mixed Ogden type hyper-elastic models of strain energy density are used to represent passive tissue properties, and active con- traction in muscle fibers is emulated by changing externally specified activation parameters in an anisotropic component of the strain energy density. The activation can vary over time and in its spatial distribution over a muscle, which is accomplished by specifying center point and spread of a Gaussian distribution in the muscle region. The tongue model is realized as a finite element implementation with large displacement/large strain calculations using incompressible 8-node elements in a commercial FE code (Marc by MSC-Software).

1pSCc52. Stable patterns of articulatory movements across inter-subject variability. Patrizia Bonaventura (Case Western Reserve University, 11206 Euclid Ave, Room 405, Cleveland, OH 44106, USA, pxb72@case.edu), Paul Taylor (Case Western Reserve University, 11206 Euclid Ave, Room 405, Cleveland, OH 44106, USA, paul.taylor@ case.edu)

The goal of the present study was to test whether models of portions of curves, representing movements of the crucial articulator for production of place in syllables containing labiodental and alveolar consonants ('iceberg' portions of demisyllables), that had previously been found to be stable across different prosodic conditions (Bonaventura, 2003,2005, 2006; Bonaventura and Fujimura, 2007), a) remained stable across different subjects pronunciations, for each consonantal class b) were significantly different for the two different consonantal movements. Curves were previously extracted from microbeam articulatory data. Curve fitting models were obtained, by using a best fit fourth order polynomial, from 592 curves representing lower lip displacement for production of [f] in 'five' (3 subjects) and from 299 curves representing tongue tip displacement for production of [n] in 'nine' (3 subjects). Coefficients were statistically compared; results showed a) significant difference for all coefficients between subjects pronouncing same consonant, except for the 3rd coefficient, both in initial and final demisyllable, representing stability of slope around a position threshold. These results might indicate stable patterns across inter-subject variability; b) coefficients were significantly different for [f] vs [n] models across speakers, within initial vs. final demisyllable, possibly indicating properties of an identifiable articulatory gesture.

\title{
Session 1pSPa
}

\section{Signal Processing in Acoustics: Sound Reproduction and Source Separation}

\author{
William L. Martens, Cochair \\ McGill University, Schulich School of Music, 555 Sherbrooke Street West, Montreal, QC H3A 1E3, Canada
}

Jamie A. Angus, Cochair

University of Salford, 12 Lister Way, York, YO30 6NL, UK

\section{Contributed Papers}

\begin{abstract}
1:00
1pSPa1. HRTF modeling in due consideration variable torso reflections. Markus Guldenschuh (Institute of Electronic Music and Acoustics, Inffeldgasse 10/3, 8010 Graz, Austria, markusgu@sbox.tugraz.at), Alois Sontacchi (Institute of Electronic Music and Acoustics, Inffeldgasse 10/3, 8010 Graz, Austria, sontacchi@iem.at), Franz Zotter (Institute of Electronic Music and Acoustics, Inffeldgasse 10/3, 8010 Graz, Austria, zotter@iem.at), Robert Höldrich (Institute of Electronic Music and Acoustics, Inffeldgasse 10/3, 8010 Graz, Austria, hoeldrich@iem.at)
\end{abstract}

Head related transfer functions (HRTFs) describe the physical path from an acoustical source to the ears. It can be gained within the relation of two measurements. The first will give the reference sound pressure in the virtual middle of the head the second has to be done in both ears. In literature exhaustive investigations concerning the idealized location of the second position can be found. Beyond that measurements with various artificial heads and human-beings considering various source directions (azimuth and elevation) with different angular resolutions have been undertaken. The obtained HRTFs exhibit, with subject to the examined source position, frequency dependent magnitude and phase. These complex transfer functions are caused by various reflections, diffractions, pressure superelevation, and shadowing, respectively. Reflections are caused form pinna, head and torso, too. Heretofore during determination of the transfer functions the influence of various head-torso arrangements have not be considered. Within this paper the differences which will be caused due to different head-torso angles will be highlighted. Beyond that a numerical model based on interpolation using spherical harmonics will be presented. By the aid of that model the 
integration within an existing binaural resynthesis model can be realized and perceptual differences within a dynamic system can be investigated.

$1: 20$

1pSPa2. Soundscape source extraction using wavelet-based sparse representations. Oliver Bunting (University of York, Department of Electronics, Heslington, YO10 5DD York, UK, ob103@ohm.york.ac.uk), David Chesmore (University of York, Department of Electronics, Heslington, YO10 5DD York, UK, edc1@ohm.york.ac.uk)

As part of the EPSRC Instrument for Soundscape Recognition, Identification and Evaluation (ISRIE) project, the work described here aims to extract sources from a soundscape, with the aim of improving the signal to interference ratio to aid automatic identification and classification. The paper will describe the project's background and aim. Results using directional audio coding (dirAC) to separate sparse signals will be presented. This method has been shown to have successfully separated two speakers at known positions from a virtual B-format soundscape. An extension of this method using the dual-tree complex wavelet transform will also be presented, which improves the sparsity of the time-frequency representation. Initial results for spatially known sources will be presented. It is expected that some results from current work on localising and tracking sources in a B-format soundscape will be presented.

\section{$1: 40$}

1pSPa3. Instrument for Soundscape Recognition, Identification and Evaluation (ISRIE): Signal Classification. Jon Stammers (University of York, Department of Electronics, Heslington, YO10 5DD York, UK, js185@york.ac.uk), David Chesmore (University of York, Department of Electronics, Heslington, YO10 5DD York, UK, edc1@ohm.york.ac.uk)

ISRIE is a collaborative project between the universities of York and Newcastle and ISVR in Southampton. The work being undertaken at York is in its second year and focuses on signal separation and classification. Developing novel methods for classifying urban and other sounds into distinct categories (such as transportation, industrial, human, animal, etc.) is the focus of this project. The classification system will consist of two main parts: a feature extractor and a classifier. Both time and time-frequency domain techniques have been considered for feature extraction and it is anticipated that a combination of domains will be required for this complex task. To date only simple classifiers have been implemented and development is now focussed on syntactic pattern recognition. The project background and an overview of the techniques involved will be presented along with preliminary results for the classification of urban and other sounds using syntactic methods.

\section{2:00}

1pSPa4. Real-time acoustic blind signal separation system based on the spatio-temporal gradient analysis. Kenbu Teramoto (Saga University, 1-Honjo, 8408502 Saga, Japan, tera@me.saga-u.ac.jp), Md. Tawhidul Islam Khan (Saga University, 1-Honjo, 8408502 Saga, Japan, khan@me.saga-u.ac .jp)

This paper presents an autonomous directivity microphone system for the blind source separation based on the newly proposed spatio-temporal gradient algorithm. The blind source separation technique principally uses no a priori knowledge about parameters of convolution, filtering, nor mixing. In the simplest case of the blind source separation problems, the observed mixing signals are the linear combinations of unknown source signals which are mutually and statistically independent and contain zero mean. The proposed blind signal separation algorithm utilizes the linearity among the four signals: (1) the sound pressure, (2) x, (3) y, and (4) z-directional particle velocities, all of which are governed by the wave equation and the equation of motion. The proposed method, therefore, has an ability to simplify the convoluted blind source separation problems into the instantaneous blind source separation problems over the spatio-temporal gradient space. Several acoustical experiments have been performed with acceptable performance of the proposed method of blind source separation. A sensor for measuring the particle velocity of acoustic signal has been used in the experiment.

MONDAY AFTERNOON, 30 JUNE 2008

ROOM 343, 4:00 TO 7:00 P.M.

\title{
Session 1pSPb
}

\section{Signal Processing in Acoustics: Acoustical Nondestructive Evaluation, Ultrasonics, and Imaging I}

\author{
Grace A. Clark, Cochair \\ Lawrence Livermore National Laboratory, 7000 East Ave., L-130, Livermore, CA 94550, USA \\ Ning Xiang, Cochair \\ Rensselaer Polytechnic Institute, Greene Building, School of Architecture, 110 8th Street, Troy, NY 12180, USA
}

\section{Contributed Papers}

\begin{abstract}
4:00
1pSPb1. Signal analysis in acoustic microscopy for nondestructive inspection of varnish layers on metal substrates. Sebastian Brand (University of Halle, Magdeburger St. 22, 06112 Halle, Germany, Sebastian.Brand@medizin.uni-halle.de), Peter Czuratis (SAM TEC GmbH, Gartenstrasse 133, $0553 \quad$ Aalen, Germany, p.czurratis@stec-germany.com), Kay Raum (Martin Luther University of Halle-Wittenberg, Dept. of Orthopedics, Q-BAM Group, Magdeburger Str. 22, 06097 Halle, Germany, kay.raum@medizin.uni-halle.de)

For preventing corrosion and for surface protection metallic objects are commonly finished with layers of varnish. The integrity of the varnish and potential defect propagation influence the durability of the metal and hence are a measure for the quality of the finishing. Scanning acoustic microscopy provides high axial and lateral resolution, a sufficient penetration depth and
\end{abstract}

is nondestructive. The goal of this work was the development of a method for detection and evaluation of delaminations of varnish layers on metallic surfaces. Investigated were samples containing one and two layers of varnish. One group contained priming only whereas the second group contained varnish on top of the undercoat. The surface integrity of the finishing was destroyed by a scratch through all finishing layers. Defect aging was then performed by exposing the samples to a corrosion-friendly atmosphere. Scanning acoustic microscopy combined with signal analysis was performed for investigating the connectivity between the finishing layers and the substrate. A robust numerical deconvolution technique has been adapted and optimized to enable the separation of strongly overlapping pulses. Echoes originated at the substrate and the finishing layers have been localized and layer thicknesses/distances were estimated. Delaminated spots of the finishing were successfully evaluated using the method developed during this study. 


\section{4:20}

1pSPb2. Structural noise characterization and flaw detection in austenitic stainless steels using ultrasonic signals, wavelet analysis and significance testing. Mohamed Khelil (ENSIM - LAUM, Université du Maine, rue Aristote, 72085 Le Mans, France, mohamed.khelil@univ-lemans.fr), Jean-Hugh Thomas (ENSIM - LAUM, Université du Maine, rue Aristote, 72085 Le Mans, France, jean-hugh.thomas@univ-lemans.fr), Rachid El Guerjouma (ENSIM LAUM, Université du Maine, rue Aristote, 72085 Le Mans, France, Rachid.El_Guerjouma@univ-lemans.fr), Laurent Simon (LAUM, CNRS, Université du Maine, Lab. d'Acoustique Université du Maine, UMR CNRS 6613, 72085 Le Mans Cedex 9, France, Laurent.Simon@univ-lemans.fr), Malika Boudraa (USTHB Faculté d'électronique et d'informatique, BP 32, El-Alia, 16031 Alger, Algeria, mk .boudraa@yahoo.fr)

The aim of this study is to characterize the structural noise in order to better detect flaws in several heterogeneous materials (steels, welding, composites $œ$ ) using ultrasonic waves. For this purpose, a continuous wavelet transform is applied to ultrasonic Ascan signals acquired using an ultrasonic Non Destructive Testing (NDT) device. The time-scale representation provided, which highlights the temporal evolution of the spectral content of the Ascan signals, is relevant but can lead to misinterpretation. The problem is to identify if each pattern from the wavelet representation is due to the structural noise or a flaw. To solve it, a detection technique based on statistical significance testing in the time-scale plane is used. Typical structural noise signals are then described using an autoregressive model which seems relevant according to the spectral content of the signals. The approach is tested on experimental signals, obtained by ultrasonic NDT of metallic materials (austenitic stainless steel) then of a welding in this steel and indeed enables to separate various components from the signal that is two kinds of structural noises and flaw echoes.

\section{$4: 40$}

1pSPb3. Flaw detection and thin materials thickness measurement using time frequency and high resolution algorithms. Redouane Drai (Image and signal processing laboratory. Welding and NDT Centre, Route de Dely-Ibrahim, BP 64, Chéraga, 16035 Alger, Algeria, drai_r@yahoo.fr), Mohamed Khelil (ENSIM - LAUM, Université du Maine, rue Aristote, 72085 Le Mans, France, mohamed.khelil@univ-lemans.fr), Fouzia Megherbi (Welding and Control Research Center, Route de Dely-Ibrahim, B.P 64, Chéraga, 16035 Algiers, Algeria, fmegherbi@yahoo.fr), Abdelsaalam Benammar (Image and signal processing laboratory. Welding and NDT Centre, Route de Dely-Ibrahim, BP 64, Chéraga, 16035 Alger, Algeria, abs_benammar@yahoo.fr)

Several techniques of signal processing were introduced in ultrasonics NDT field. In thin samples the reflected signals are overlapping thus making detection of defects in these samples and accurate measurements impossible. It is thus necessary to enhance the visibility of the defect echo by signal processing techniques. In this context, we develop signal processing tools allowing detecting and locating the imperfections present in these materials. In this paper, we contribute by the development of some signal processing techniques based on time frequency and high resolution algorithms in order to enhance the resolution of flaw detection and to measure thin materials thickness. 1- We propose to implement temporal versions of methods known as high resolution like MUSIC, Root MUSIC and Eigen vectors method. These methods allow frequencies extraction in the case of the complex signals drowned in noise. 2- We apply time-frequency algorithms based on STFT, Wigner-Ville, Gabor transform on thin materials thickness measurement. A comparative study is carried out between all of these algorithms and is applied in separation of closer flaw echoes and thin materials thickness measurement. Satisfactory results are obtained with Gabor transform in measurement of few tenth $(0.1) \mathrm{mm}$.

\section{5:00}

1pSPb4. Investigation of damage mechanisms of composite materials: multivariable analysis based on temporal and wavelet features extracted from acoustic emission signals. Anne Marec (ENSIM - LAUM, Université du Maine, rue Aristote, 72085 Le Mans, France, anne.marec.etu@univ-lemans.fr), Jean-Hugh Thomas (ENSIM - LAUM, Université du Maine, rue Aristote, 72085 Le Mans, France, jean-hugh.thomas@univ-lemans.fr), Rachid El Guerjouma (ENSIM LAUM, Université du Maine, rue Aristote, 72085 Le Mans, France, Rachid .El_Guerjouma@univ-lemans.fr)

The aim of this work is the analysis of damage development and timeto-failure mechanisms within fibre-matrix composite materials in order to assess their remaining lifetime using in situ ultrasonic sensors. Thus Acoustic Emission (AE), which represents the generation of transient ultrasonic waves in a material under load, is used to discriminate the different damage mechanisms in composite materials. In this work, unsupervised pattern recognition analyses (fuzzy c-means) associated with a principal component analysis are used for classifying the $\mathrm{AE}$ events. A cluster analysis of $\mathrm{AE}$ data is achieved and the resulting clusters are correlated to the damage mechanisms of the material under investigation. This method gives good results on complex composite materials such as glass fibre/polyester cross-ply composites, sheet molding compound (SMC), concretes. Furthermore, AE signals of heterogeneous materials are not stationary. Thus, time-scale or timefrequency methods (continuous and discrete wavelet transforms) are used to determine new relevant descriptors from AE signals. These new descriptors are introduced in the clustering process to provide a better characterization and discrimination of the different damage mechanisms.

\section{$5: 20$}

1pSPb5. Ultrasonic structural health monitoring by multiple-input-single-output minimum variance processing. Jeffrey Krolik (Duke University, ECE Dept. Hudson Hall 130, P.O. Box 90291 , Durham, NC 27708, USA, jk@ee.duke.edu), Granger Hickman (Duke University, ECE Dept. Hudson Hall 130, P.O. Box 90291, Durham, NC 27708, USA, gwh@ee.duke.edu), Lawrence Carin (Duke University, ECE Dept. Hudson Hall 130, P.O. Box 90291, Durham, NC 27708, USA, lcarin@ee.duke.edu), Jennifer E. Michaels (Georgia Institute of Technology, Van Leer Electrical Engineering Building, Atlanta, GA 30332, USA, jennifer.michaels@ece.gatech.edu)

This work concerns ultrasonic detection and localization of defects in platelike metal structures such as airfoils. In previous work, MIMO delayand-sum beamforming has been used to image these defects using changedetected ultrasonic signals between a sparse array of transmitters and receivers. However, mismatch of medium dispersion and unknown scattering properties of the defects can lead to significant imaging artifacts. To reduce imaging artifacts and sidelobes, a multiple-input-single-output (MISO) minimum variance (MV) approach is proposed here. MV-MISO imaging involves electronically scanning a transmit beam over the area of interest while, at each point being imaged, virutal transmit nulls are adaptively steered in the directions of interfering scatterers. For each imaging cell, this is achieved by adjusting transmit weights to minimize the variance of signals received at a single receiver subject to the constraint of achieving constant insonification. Using a model of dispersive Lamb wave propagation, the proposed algorithm has been successfully applied to change-detected data gathered with a six-sensor transmit-receive array coupled to a thin aluminum plate with artificial defects (e.g., drilled holes).

\section{$5: 40$}

1pSPb6. An accurate distance measurement by calibration of doppler-shift for ultrasonic sonar sensing. Hirata Shinnosuke (Tokyo Institute of Technology, 4259 Nagatutamati, Midori-ku, 226-8502 Yokohama, Japan, hirata.s.ab@m.titech.ac.jp), Minoru K. Kurosawa (Tokyo Institute of Technology, 4259 Nagatutamati, Midori-ku, 226-8502 Yokohama, Japan, mkur@ip.titech.ac.jp), Takasi Katagiri (Sutekina Inc., 20014 Akaho, 3994117 Komagane, Japan, takashi.katagiri@ primemotion.com)

Ultrasonic distance measurement with the pulse-echo method is based upon the determination of the time of flight of ultrasonic waves. The pulsecompression technique, which involves the calculation of the cross- 
correlation function between the transmitted and the reflected waves, is the conventional method for improving the resolution of the measurement. The calculation of a cross-correlation operation requires high-cost digital signal processing, however. This paper presents a new sensor signal processing method of the pulse-compression technique using a delta-sigma modulated single-bit digital signal. The proposed method consists of three parts: an analyzing operation of the doppler-shifted frequency, a cross-correlation operation by single-bit signal processing, and a smoothing operation accomplished by a moving average filter. For the pulse-compression technique, this method reduces the calculation cost of digital signal processing. Since the frequency of the linear chirp signal is doppler-shifted, the distance from the moving transmitter to the moving object cannot be accurately measured in the pulse-compression technique. To solve this problem, the analyzing operation of the doppler-shifted frequency is used to calibrate the transmitted ultrasonic wave, thus effectively improving the accuracy of the measurement.

\section{6:00}

1pSPb7. Image processing and wavelets transform for sizing of weld defects using ultrasonic TOFD images. Ahmed Kechida (Image and Signal Processing Laboratory. Welding and NDT Centre, Route de DelyIbrahim, BP 64, Chéraga, 16035 Alger, Algeria, Abs_benammar@yahoo.fr), Redouane Drai (Image and Signal Processing Laboratory. Welding and NDT Centre, Route de Dely-Ibrahim, BP 64, Chéraga, 16035 Alger, Algeria, drai_r@yahoo.fr), Abdelsaalam Benammar (Image and Signal Processing Laboratory. Welding and NDT Centre, Route de Dely-Ibrahim, BP 64, Chéraga, 16035 Alger, Algeria, abs _benammar@yahoo.fr)

Ultrasonic time-of-flight diffraction (TOFD) is rapidly gaining prominence as a reliable nondestructive testing technique for weld inspection in steel structures, providing highly accurate positioning and sizing of flaws. A number of signal and image processing tools have been specifically developed for use with TOFD data and adapted to function autonomously without the need for continuous intervention, configuring automatically according to the nature of the data and the data acquisition settings. This paper presents the results of several innovative procedures based on Multiresolution analysis such as wavelet transforms and texture analysis for detecting edges of planar defects. The approach is based on the decomposition by packets of wavelets of the image while taking into account of the underimage content textural after each level of decomposition. The reconstruction of the image is done by eliminating the underimages of poor textural information and at the end the segmented image is got by a binarisation operation done on the image rebuilt histogram. The automation of sizing and positioning of weld flaws in TOFD data as an essential stage of a comprehensive TOFD inspection and interpretation aid is developed and implemented. Key words: U1trasonic Time-Of-Flight Diffraction., Texture Analysis, Wavelet Transform.

\section{6:20}

1pSPb8. Temporal approach of the synthetic aperture imaging using Hadamard matrix. Frédéric Mosca (Ixsea, 46 Quai François Mitterrand, 13600 La Ciotat, France, fmo@ixsea.com), Jean-Marie Nicolas (Ecole Nationale Supérieure des Télécommunication de Paris, Telecom ParisTech, Département TSI, 46 rue Barrault, 75634 Paris Cedex 13, France, nicolas@enst.fr), Laurent Kopp (IXwaves, 220 rue Albert Caquot, 06560 Sophia Antipolis, France, laurent.kopp@ixwaves.com), Mathieu Couade (Supersonic Imagine, Les Jardins de la Duranne, bât E, 510, rue René Descartes, 13857 Aix-en-Provence, France, mathieu.couade @ supersonicimagine.fr)

The synthetic aperture imaging is a very promising solution in the wellknown compromise between contrast and frame rate. Indeed this method leads to the measurement of each transmitter/receiver impulse response of the system. From this fact, synthetic aperture imaging reach the transmit/receive focus imaging quality for the cost in frame rate of the number of antenna's elements. The main inconvenient of this method is the very low signal to noise ratio provided. Indeed, using only one transmitter per sequence leads to a very poor penetration. To correct this, a method using spatial Hadamard sequences has been introduced. For each of this Hadamard sequence, a Hadamard beam is generated in the medium. By a temporal approach, some interesting properties of those beams are highlighted and a method using those properties is proposed. Some experiments have been done using those properties and the results show an important improvement of the frame rate for a very small cost in contrast.

\section{6:40}

1pSPb9. Elastic-wave reverse-time migration imaging with a new vector-imaging condition. Huseyin Denli (Los Alamos National Laboratory, Mail Stop D408, Los ALamos, NM 87544, USA, denli@lanl.gov), Lianjie Huang (EES-11 (Geophysics) - Los Alamos National Laboratory, MS D443, Los Alamos, NM 87545, USA, ljh@lanl.gov)

With increasing computing capability, the use of elastic waves in threedimensional subsurface imaging is becoming more feasible. However, the research in this area is still in the embryonic stage. In conventional seismic imaging, multicomponent data are decomposed into compressional and shear components, and solutions of the acoustic-wave equation are used to process each component independently. Such an imaging technique, however, cannot correctly handle elastic-wave conversions/couplings in complex regions, which is critically important for high-resolution and reliable imaging. In this paper, we develop elastic reverse-time migration imaging, applying a finite-difference solution to the pure elastic-wave equation in heterogeneous media. We implement numerical reverse-time migration imaging in a scheme similar to time-reversal acoustics in the laboratory. To correctly handle polarizations of compressional- and shear-waves during imaging, we also develop a novel vector-imaging condition for elastic-wave reverse-time migration. We use synthetic reflection data to demonstrate that, compared with the conventional imaging condition, our new vector-imaging condition increases image resolution and reduces image artifacts. The new imaging algorithm can significantly improve our capability to image complex subsurface structures. 


\title{
Session 1pUW
}

\section{Underwater Acoustics, Acoustical Oceanography, and ECUA: Seabed and Sea Surface Interaction Measurements and Modeling}

\author{
Peter H. Dahl, Cochair \\ Applied Physics Laboratory, University of Washington, 1013 NE 40th St, Seattle, WA 98105, USA \\ Michael A. Ainslie, Cochair \\ TNO Defence, Security and Safety, Oude Waalsdorperweg 63, The Hague, 2597 AK, Netherlands \\ Invited Paper \\ 1:00
}

1pUW1. Fine-grained sediment layers: an acoustic lens for sub-bottom sediment processes and structures. Charles W. Holland (Penn State University/Applied Research Lab, Applied Science Bldg., State College, PA 16804, USA, cwh10@psu.edu)

\begin{abstract}
In many coastal and deep water regions, there is a layer of fine-grained sediment (e.g., clay and silty-clay) over coarser grained sediments or rock. This layer may range from scales of centimeters to hundreds of meters thick. Fine-grained sediments typically have a sound speed less than that of the interstitial fluid (i.e., overlying water column) with modest attenuation and thus act as a refracting lens for the acoustic field as it interacts with sub-bottom structures. The presence of fine-grained sediments frequently opens an effective window for acoustically probing very small-scale processes within the sediment. The presence of fine-grained sediments also has a profound impact on seabed reflection and scattering, hence propagation, reverberation, and clutter. The effect of fine-grained sediment layers (along with concomitant sub-bottom processes) on reflection and scattering is discussed using both theory and measurements. [Research supported by ONR OA321.]
\end{abstract}

\section{Contributed Paper}

\section{1:20}

1pUW2. A parametric study of interactions between acoustic signals reflected by the seafloor. Samuel Pinson (Ecole Navale/Irenav, BP 600, 29240 Brest Armées, France, samuel.pinson@ecole-navale.fr), Laurent Guillon (Ecole Navale/Irenav, BP 600, 29240 Brest Armées, France, guillon @ecole-navale.fr)

This paper deals with the study of broadband acoustic signals reflected by the seafloor and recorded on a vertical array in shallow water areas. Only the first bottom reflected path is considered here. In previous studies (see e.g., Guillon and Holland, JASA, 122, 2974), two different aspects of the "coherence" of these signals were examined (the maximum of the cross- correlation coefficients and the phase of the cross-spectra) and it was shown that these parameters are sensitive to the geoacoustic nature of the seafloor. These results show that the interactions between signals can be used efficiently for improving geoacoustic inversion schemes or for transmission loss predictions. Before going to such applications, a detailed parametric study is needed to investigate the sensitivity of the interactions measurement to various parameters (range, geoacoustic structure,...). This is done using a numerical model, based on the spherical wave decomposition of plane waves, that simulates the signals reflected by the seafloor. The parametric study lead shows the relative importance of the various parameters and gives indications for the possibility of inverting these parameters.

\section{Invited Paper}

1pUW3. Mid-frequency bottom interaction measurements off the New Jersey continental shelf. Jee Woong Choi (Dept. of Environmental Marine Sciences, Hanyang Univ., 1271, Sa-3-dong, Sangrok-gu, 426-791 Ansan, Republic of Korea, choijw@ hanyang.ac.kr), Peter H. Dahl (Applied Physics Laboratory, University of Washington, 1013 NE 40th St, Seattle, WA 98105, USA,dahl@apl.washington.edu)

Two kinds of bottom interaction measurements conducted in waters $80 \mathrm{~m}$ deep off the North American continental shelf as part of Shallow Water 06 (August, 2006) are discussed. In each, acoustic signals were recorded on two, colocated vertical line arrays of length $1.4 \mathrm{~m}$, one at depth $25 \mathrm{~m}$, and other at depth $50 \mathrm{~m}$. The source was deployed at depth $40 \mathrm{~m}$ from the R/V Knorr that could either be positioned or towed at rate $0.1 \mathrm{~m} / \mathrm{s}$. The first, "bottom reflection" is interpreted as a measure of the modulus of the plane wave reflection coefficient as functions of frequency $(1-20 \mathrm{kHz})$ and grazing angle associated with the discrete set of ranges $(50-300 \mathrm{~m})$. The second represents a "move-out" type measurement with source towed away from the receiver, and reflection at continuous angles associated with the 50-300 m range. Frequency range was $1-2 \mathrm{kHz}$, and as the source was $5 \mathrm{~m}$ off the bottom, spherical wave effects are 
investigated. Both measurements were carried out over the same four directions originating from the receiver, each separated by 90 degrees. Physical processes responsible for the observations in each case are discussed and modeled. [Supported by ONR, with partial support from Hanyang University.]

\section{Contributed Paper}

\section{2:00}

1pUW4. Analysis of acoustic backscattering from the ocean bottom using radiative transfer theory. Jorge E. Quijano (NEAR Lab-Portland State University, 1900 SW 4th Avenue, Suite 160, Portland, OR 97201, USA, jorgeq@pdx.edu), Lisa M. Zurk (NEAR Lab-Portland State University, 1900 SW 4th Avenue, Suite 160, Portland, OR 97201, USA, zurkl @ cecs.pdx.edu)

Radiative Transfer (RT) theory is a heuristic formulation for analysis of wave propagation in random media based on the principle of conservation of energy rather than on the solution of the wave equation. The theory has been applied to electromagnetics and more recently to ultrasound and seismics, and it has the advantage of being computationally tractable for complex ran- dom environments. In this paper the RT formulation is proposed for the problem of layered ocean bottom sediment with random scatterers. The volume backscattering level from tenuous media is obtained by solving the RT equation at each individual layer and by applying the corresponding reflection and transmission coefficients at the rough boundaries. Simulations of the backscattered acoustic intensity from a finite layer with elastic spherical scatterers are presented. The results obtained from the RT equation are compared to those based on the wave equation. Single frequency steady state solutions are considered for different cases of sediment attenuation and layer thickness. The flexibility of the RT method is demonstrated by showing the individual effect of propagating longitudinal and shear waves in the elastic sediment. It is proved that the contribution of shear waves in consolidated sediments is considerable.

\section{Invited Paper}

$$
2: 20
$$

1pUW5. Determination of seabed scattering law from long range reverberation. Christopher H. Harrison (NATO Undersea Research Centre, Viale San Bartolomeo 400, 19138 La Spezia, Italy, harrison@nurc.nato.int)

Reverberation degrades sonar performance, and often the dominant cause in shallow water is scattering from the seabed. Usually scattering is weaker at low angles than at high whereas, in contrast, sound propagates better at low angles than at high. This, in turn, affects the range-dependence of signal-to-reverberation which influences the choice of source level. A better understanding of this scattering angle dependence (or scattering law) offers the potential of improved discrimination of targets against this reverberation background. This paper will discuss an experimental technique for deducing the scattering angle dependence and backscattering strength from long range reverberation (out to $25 \mathrm{~km}$ ) measured on a vertical line array (VLA). Well-sampled near simultaneous angle-dependent propagation measurements out to comparable ranges are used to separate the propagation effects without the need for any modeling. Applying the technique to the Malta Plateau (during BOUNDARY2003 and BOUNDARY2004), first the method appears to work, and second it suggests that the scattering law is close to Lambert when the seabed is flat.

\section{Contributed Paper}

\section{2:40}

1pUW6. Underwater stereo-photographic measurement of small scale roughness: limits to spatial correlation accuracy. Gaetano Canepa (NATO Undersea Research Centre, Viale San Bartolomeo 400, 19126 La Spezia, Italy, canepa@nurc.nato.int), Eric Pouliquen (NATO ACT, 7857 Blandy Road, Suite 100, Norfolk, 23551-2490, USA, pouliquen@act.nato.int), Anthony P. Lyons (Applied Research Laboratory, The Pennsylvania State University, P.O. Box 30, State College, 16804, USA, ap12@psu.edu)

In recent years stereo-photogrammetry measurements of seafloor small scale roughness have been reported in the literature. Stereo-photogrammetry algorithm consists of a comparison between areas (windows) belonging to corresponding regions of a pair of photos. This comparison may be per- formed numerically or by human eyes using a photogrammetric stereocomparator. The results of this process are then converted in an array of seafloor heights. Until now underwater stereo-photographic data are given with no quantitative measurement of the positions and position correlation lengths errors: this work addresses this problem applying the results to the SAPHO system (Seafloor Automatic PHOtogrammetry) developed at NURC. It is shown that positional error estimates for stereo-photographic systems must take into account not only line/matrix resolution and system geometry but also the effects of comparison window size and image quality. A method by which this can be achieved is described. It is also shown that the stereo-photographic systematic error has an important effect when the purpose of the measurement is to determine seafloor correlation length or power spectral density for acoustic scattering estimation.

\section{Invited Paper}

1pUW7. Bubbles at the top and bottom of the water column: the acoustical effects, and the use of acoustics to measure them. Timothy G. Leighton (Institute of Sound and Vibration, Univ. of Southampton, University Road, Highfield, SO17 1BJ Southampton, UK, T.G.Leighton@soton.ac.uk), David Coles (National Physical Laboratory, Hampton Road, TW11 OLW Teddington, UK, dc@isvr.soton.ac.uk), Michael A. Ainslie (TNO Defence, Security and Safety, Oude Waalsdorperweg 63, 2597 AK The Hague, Netherlands, michael.ainslie@tno.nl), Paul R. White (Institute of Sound and Vibration, Univ. of Southampton, University Road, Highfield, SO17 1BJ Southampton, UK, prw@isvr.soton.ac.uk)

Bubbles of atmospheric gas can be entrained by breaking waves at the top of the water column: this bubble population is dynamic, and will evolve through the effects of buoyancy, gas exsolution and dissolution, and the fragmentation and coalescence of bubbles. These processes are important to ambient noise, sonar operation, and the overall gas budget of the planet. At the base of the water column, methane bubbles can occur in marine sediments, a phenomenon important to the global methane budget, to the petrochemical industry, and to the stability of the sediment (e.g., for civil engineering purposes). This paper examines the acoustical effects of both of 
these populations, and the ways in which acoustics can be used to measure them. Data will be presented from field trials, including measurements of gassy marine sediments in UK waters, and of wave-generated bubble clouds measured by an $11 \mathrm{~m}$ spar buoy deployed from 16th June to 18th July 2007 at a distance of 400 miles off the west coast of Portugal.

\section{3:20-3:40 Break}

\section{Contributed Paper}

3:40

1pUW8. The effect of wind-generated bubbles on sea-surface backscattering at $940 \mathrm{~Hz}$. Robbert Van Vossen (TNO Defence, Security and Safety, Oude Waalsdorperweg 63, 2597 AK The Hague, Netherlands, robbert.vanvossen@tno.nl), Michael A. Ainslie (TNO Defence, Security and Safety, Oude Waalsdorperweg 63, 2597 AK The Hague, Netherlands, michael.ainslie@tno.nl)

Predictions of sea-surface backscattering strength are needed for sonar performance modelling. Such predictions are hampered by two problems. First, measurements on surface backscattering are not available at small grazing angles. These are of special interest to low-frequency active sonar since these mainly contribute to long range propagation. Second, existing theoretical models underestimate the surface backscattering strength at larger grazing angles. We investigate whether wind-generated bubbles can explain this deficit. Thus, we develop a theoretical description which includes the effect of refraction and scattering of sound by wind-generated bubbles. We compare the theoretical surface backscattering predictions to Critical Sea Test measurements. These are a set of backscattering measurements for different wind speeds, and grazing angles are in the range between 5 and 30 degrees. The comparison shows that a good fit can be obtained between the theoretical predictions and the surface backscattering measurements, depending on the population density of resonant bubbles. This indicates that wind-generated bubbles significantly contribute to rough-surface back-scattering. The bubble backscattering contribution is dominant at large wind speeds and also at small grazing angles.

\section{Invited Paper}

\section{4:00}

1pUW9. Quasi-deterministic forward scattering from the sea surface. Grant Deane (Scripps Inst. Oceanography, Univ. Califiornia, San Diego, La Jolla, CA 92093, USA, gdeane@ucsd.edu), James Preisig (Woods Hole Oceanographic Institution, Bigelow 404, MS\#9, Woods Hole, MA 02543, USA, jpreisig@whoi.edu), Chris T. Tindle (Physics Department, University of Auckland, Bag 92019, 1010 Auckland, New Zealand, c.tindle@auckland.ac.nz)

Gravity waves create focal regions and caustics in sound forward-scattered from the sea surface. Focal regions are associated with rapid variations in amplitude and Doppler shift, which can impact the performance of underwater communications systems and sonars operated near the shore. Single-bounce focal regions consist of a superposition of up to 3 micro-paths, each of which is associated with its own amplitude, time delay, and Doppler shift. Focal regions move systematically through the water column as the wave propagates. Studies of these effects in near-shore ocean experiments and a laboratory flume have shown that a quasi-deterministic analysis of wave scattering can lead to valuable insights about the underlying scattering process. [Work supported by ONR.]

\section{Contributed Papers}

\section{4:20}

1pUW10. Multipath reflection from surface waves. Chris $T$. Tindle (Physics Department, University of Auckland, Bag 92019, 1010 Auckland, New Zealand, c.tindle@auckland.ac.nz), Grant Deane (Scripps Inst. Oceanography, Univ. Califiornia, San Diego, La Jolla, CA 92093, USA gdeane@ucsd.edu), James Preisig (Woods Hole Oceanographic Institution, Bigelow 404, MS\#9, Woods Hole, MA 02543, USA, jpreisig@whoi.edu)

A tank experiment was conducted at Scripps Institution of Oceanography to measure reflection of underwater sound from surface waves. Shor pulses at a nominal $200 \mathrm{kHz}$ were transmitted beneath surface waves of wavelength $0.7 \mathrm{~m}$ to a receiver at $1.2 \mathrm{~m}$ range. The surface wave crests act as curved mirrors for underwater sound and lead to focussing and caustics in the surface reflected pulses. The locations of the foci and caustics move steadily as the wave progresses and lead to rapid variation of amplitude, phase and arrival time of the received pulses. Wavefront modeling has been used to calculate theoretical waveforms for the measured surface wave shape. The theory shows there are typically three distinct reflected eigenrays beneath a wave crest and they interfere to give rapid variation of the received signal. The theory gives good agreement with the details of the time dependent interference of the surface reflected pulses. [Work supported by ONR.]

\section{4:40}

1pUW11. Observations and modeling of angular compression and vertical spatial coherence in sea surface forward scattering. Peter $\mathrm{H}$. Dahl (Applied Physics Laboratory, University of Washington, 1013 NE 40th St., Seattle, WA 98105, USA, dahl@apl.washington.edu)

Measurements and modeling of vertical spatial coherence and related angular spreading associated with forward scattering from the sea surface are presented. The van Cittert-Zernike theorem is used in the modeling. The measurements were conducted in waters $80 \mathrm{~m}$ deep off the North American continental shelf (Shallow Water 06, Aug. 2006). Acoustic signals were recorded on a moored array system (MORAY) consisting of three arrays (each $1.4 \mathrm{~m}$ ): two vertical line arrays at depth $25 \mathrm{~m}$ and $50 \mathrm{~m}$, and one horizontal array at depth $27 \mathrm{~m}$. The source $(1-20 \mathrm{kHz})$ was deployed at depth $40 \mathrm{~m}$ from the R/V Knorr, at ranges 50-500 m from the MORAY. Conditions were characterized by a downward-refracting profile, e.g., sound speed going from $1530 \mathrm{~m} / \mathrm{s}$ at the surface to $1490 \mathrm{~m} / \mathrm{s}$ at depth $25 \mathrm{~m}$. Refraction modifies the vertical angular vertical spread due to scattering, which can understood from Snell's law. The Snell mapping is smooth, and a Taylor expansion around the mean grazing angle provides a functional relation between the angular variance near the surface and that at the receiver associated with scattering. The latter is measurably compressed owing to refraction, an effect mirrored in the spatial coherence data. [Research support from ONR.]

\section{5:00}

1pUW12. Unperturbed normal mode method for forward surface scattering. Frank S. Henyey (Applied Physics Laboratory, University of Washington, 1013 NE 40th St, Seattle, WA 98105, USA, frank@apl.washington.edu), Eric Thorsos (Applied Physics Laboratory, University of Washington, 1013 NE 40th St, Seattle, WA 98105, USA, eit @apl.washington.edu)

A technique is presented that uses an expansion in unperturbed modes to calculate acoustic scattering from ocean surface waves in a shallow water waveguide. The basic formalism as well as a useful extension to account for the difference between the water column and the domain in which the modes 
are calculated. The coupling between the modes due to the waves is local at the ocean surface, unlike the coupling of local modes. Numerical examples of the calculation are given for both a sinusoid surface wave and a random surface wave with a typical wind driven spectrum.

\section{$5: 20$}

1pUW13. Sonic-boom noise under a wavy air-water interface. Adam M. Fincham (University of Southern, Aerospace \& Mechanical Engineering, 854 W Downey Way, Los Angeles, CA 90089-1191, USA, afincham@usc.edu), Tony Maxworthy (University of Southern, Aerospa \& Mechanical Engineering, 854 W Downey Way, Los Angeles, CA 900891191, USA, maxworth@usc.edu), Hsien K. Cheng (University of Southern, Aerospace \& Mechanical Engineering, 854 W Downey Way, Los Angeles, CA 90089-1191, USA, afincham@usc.edu), Charles P. Griffice (The Aerospace Corporation, System Planning and Engineering, El Segundo, CA 90245-4691, USA, Charles.P.Griffice@aero.org), Johnson C. Wang (The Aerospace Corporation, System Planning and Engineering, El Segundo, CA 90245-4691, USA, Johnson.C.Wang@aero.org), John R. Edwards (U. S. Air Force, Air Force Space Command, 483 N. Aviation Blvd., El Segundo, CA 90245-2808, USA, John.Edwards@LOSANGELES.AF.MIL), Adel A.
Hashad(U. S. Air Force, Air Force Space Command, 483 N. Aviation Blvd., El Segundo, CA 90245-2808, USA, Adel.Hashad@LOSANGELES.AF .MIL)

Recent investigations of the underwater sound field produced by sonic booms show that the latter's interaction with surface waves can strongly alter the sound level, frequency range, and signal waveform reaching the deeper part of the ocean. Unlike conclusions from studies based on the flatocean model of Sawyers [1] which indicate little effects at depth beyond one signature length, the interaction of sonic-boom waves with a surface-wave train can generate nonevanescent, downward propagating waves which, owing to their lower spatial-attenuation rate, overwhelm the otherwise primary (flat-ocean) wave field. Theoretical results [2] substantiate and elucidate the significant differences between flat and wavy surfaces in waveform characteristics and their audibility in deep, and moderately deep water. Here we report on an extensive series of laboratory experiments performed specifically to validate this theoretical model and to ascertain the distinct differences in wave-field characteristics between a wavy and flat interface. The experimental measurements confirm the theoretical findings, showing the predicted differences between the sound fields measured under wavy and flat air-water interfaces stimulated by a passing sonic boom. [1] Sawyers, K. H. J. Acoust. Soc. Am., 44(2), 523 - 524, (1968) [2] Cheng, H. K. and Lee, C. J., J. Fluid Mech. 514, 281-312 (2004). 